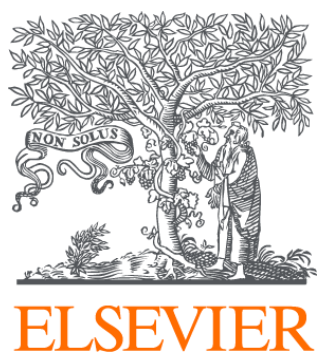

Since January 2020 Elsevier has created a COVID-19 resource centre with free information in English and Mandarin on the novel coronavirus COVID-

19. The COVID-19 resource centre is hosted on Elsevier Connect, the company's public news and information website.

Elsevier hereby grants permission to make all its COVID-19-related research that is available on the COVID-19 resource centre - including this research content - immediately available in PubMed Central and other publicly funded repositories, such as the WHO COVID database with rights for unrestricted research re-use and analyses in any form or by any means with acknowledgement of the original source. These permissions are granted for free by Elsevier for as long as the COVID-19 resource centre remains active. 


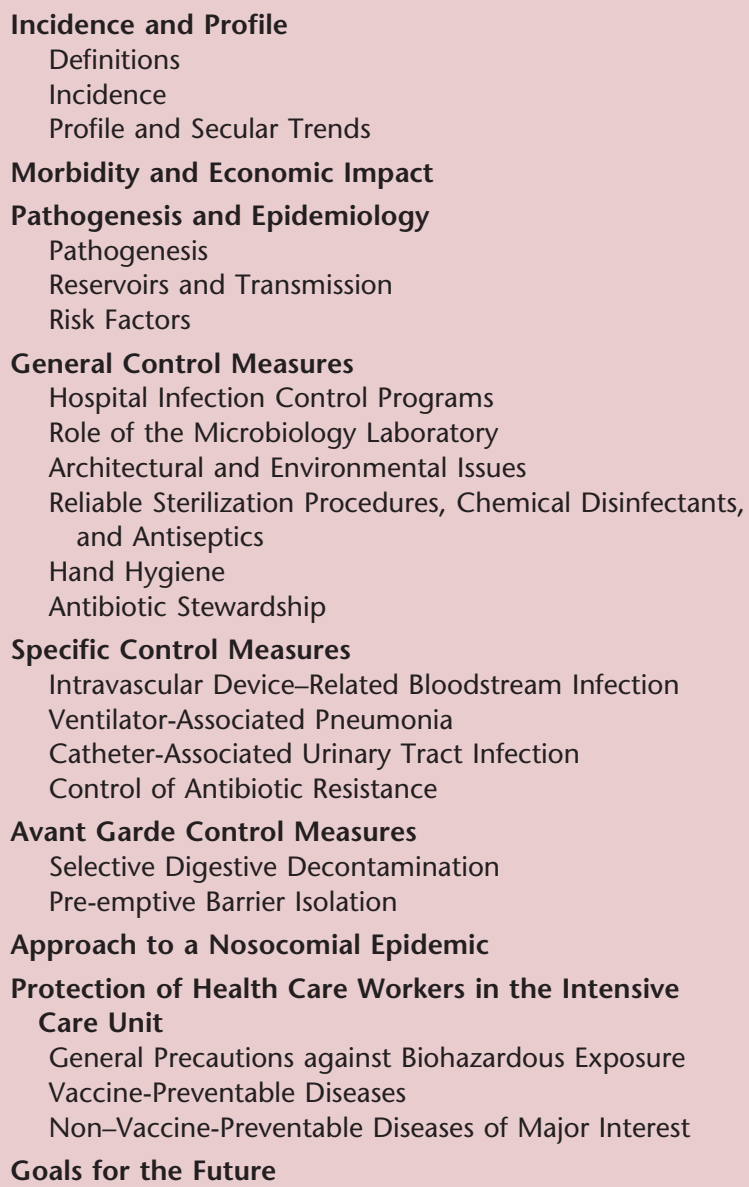

Intensive care units (ICUs) have contributed greatly to the survival of patients with trauma, shock states, and other life-threatening conditions ${ }^{1-3}$ but are associated with a greatly increased risk of nosocomial (hospital-acquired) infection. Rates of nosocomial infection in patients requiring more than 1 week of advanced life support within an ICU are three to five times higher than in hospitalized patients who do not require ICU care. ${ }^{4-8}$ Infection, usually nosocomial, is the most common cause of death, directly or indirectly, of patients who survive the early period after major trauma or full-thickness burns and is the most commonly identified cause of multiple-organ dysfunction syndrome. ${ }^{9-11}$

Although most of this book focuses on the diagnosis and management of critically ill patients in the ICU, nosocomial infections are clearly one of the most common and serious complications of ICU care and are usually a consequence of invasive monitoring or life support therapies. Thus they are greatly preventable, and it is appropriate that measures to prevent nosocomial infections be addressed.

Much has been learned over the past decade about the epidemiology of nosocomial infection acquired in the ICU. Published guidelines for prevention are now available, based increasingly on randomized trials that have established the efficacy of specific control measures. Knowledge and technology of asepsis with regard to surgery and high-risk medical devices are now sufficiently advanced that, if applied consistently, the risk of nosocomial infection can be greatly reduced. ${ }^{12-15}$

\section{INCIDENCE AND PROFILE}

\section{Definitions}

Obtaining meaningful data on rates of nosocomial infection that can form the basis for comparisons within a hospital and, especially, among hospitals and that can also be used to monitor secular trends and document the efficacy or lack of efficacy of control measures must begin with clear, unambiguous definitions. Although there are no standardized definitions for infection at specific sites that are universally accepted by clinicians or investigators, the Centers for Disease Control and Prevention (CDC) has published definitions for the purpose of surveillance 
of nosocomial infection within hospitals, which most U.S. centers and an increasing number of hospitals around the world have adopted (Box 51-1) ${ }^{16}$ For research purposes, more stringent definitions for specific infections will usually be necessary, ${ }^{17}$ especially for pneumonia. ${ }^{18}$

\section{Incidence}

The incidence of hospital-acquired infection is most commonly expressed as the number of infections per 100 patients hospitalized and is highest in burn units, ${ }^{7,19}$ surgical ICUs, ${ }^{5-7,19-22}$ and ICUs for low-birth-weight neonates $(5 \%$ to $30 \%),{ }^{4,23,24}$ with intermediate risk in medical ICUs ${ }^{4,5,7,19,22,25}$ and pediatric ICUs ${ }^{4}(5 \%$ to $7 \%)$ and lowest risk in coronary care units ( $1 \%$ to $2 \%$ ) (Table $51-1){ }^{4,7,8,19}$

Recognizing that the risk of nosocomial infection within ICUs is heavily influenced by the length of stay and that the length of stay ranges widely among ICUs in the same hospital and among different hospitals, ${ }^{26}$ the CDC has advocated the use of rates expressed per 1000 patient-days to permit more meaningful intrainstitutional and, especially, interhospital comparisons. ${ }^{26,27}$ Furthermore, recognizing the powerful influence of exposure to invasive devices on susceptibility to infection ${ }^{28,29}$ and the great variation in use of devices among different ICUs in the same hospital and among different hospitals, ${ }^{26}$ the $\mathrm{CDC}$ has further recommended surveillance of device-associated nosocomial infections expressed as infections per 1000 device-days. ${ }^{26,27}$ Representative rates of device-associated nosocomial infectionin U.S. hospitals that are members of the CDC's National Nosocomial Infection Surveillance System (NNIS), ${ }^{30}$ which can be used for intrahospital and interhospital comparisons are shown in Table 51-2. In the future, device-associated infection rates will be sought in accreditation reviews by the Joint Commission on the Accreditation of Healthcare Orga- nizations (JCAHO) $)^{31}$ as this influential organization continues to move toward measurement of patient outcomes as the most effective way to improve patient care in the United States.

\section{Profile and Secular Trends}

Approximately $40 \%$ of endemic nosocomial infections within ICUs are catheter-related urinary tract infections, and $25 \%$ are pneumonias-most associated with endotracheal intubation and mechanical ventilatory support. Up to $10 \%$ of patients hospitalized in a medical-surgical ICU for more than 72 hours acquire a nosocomial bloodstream infection, most commonly from an intravascular device. ${ }^{26,32,33}$ Postoperative surgical site infections and intra-abdominal infections; nosocomial bacteremias; and gastrointestinal infections, especially antibioticassociated Clostridium difficile colitis, ${ }^{34}$ account for the remainder. ${ }^{4-8,26}$

Nearly $50 \%$ of nosocomial infections in the ICU are caused by aerobic gram-negative bacilli, especially Pseudomonas aeruginosa, Enterobacter species, or Serratia marcescens; and $35 \%$ are caused by gram-positive cocci, most commonly coagulase-negative staphylococci or Staphylococcus aureus or, increasingly, resistant enterococci (Fig. 51-1). ${ }^{35}$ Almost $15 \%$ are caused by Candida species, ${ }^{35}$ but filamentous fungi such as Aspergillus and Zygomycetes are being increasingly encountered in patients with hematologic malignancy or those who received solid organ transplants. ${ }^{36-38}$ Viruses such as respiratory syncytium virus (RSV) ${ }^{39}$ and rotaviruses ${ }^{40}$ are important pathogens in pediatric ICUs. Legionella species now account for up to $10 \%$ of nosocomial pneumonias in centers that make efforts to diagnose Legionella infections. $^{41}$

\begin{tabular}{|c|c|c|c|c|c|c|c|}
\hline \multirow[b]{2}{*}{ Authors } & \multirow[b]{2}{*}{ Study Period } & \multicolumn{6}{|c|}{ Rate (Per 100 Discharges) by Type of ICU } \\
\hline & & Neonatal & Pediatric & Coronary & Medical & Surgical & Burn \\
\hline Hemming et al. ${ }^{599}$ & $1970-1974$ & 24.3 & & & & & \\
\hline Northey et al. ${ }^{20}$ & $1972-1973$ & & & & & 27.3 & \\
\hline Daschner et al. ${ }^{22}$ & 1976-1979 & & & & 3.6 & 35.3 & \\
\hline Caplan and Hoyt ${ }^{21}$ & $1977-1978$ & & & & & 50.9 & \\
\hline Goldmann et al. ${ }^{23,24}$ & $\begin{array}{l}1977-1979 \\
1980-1981\end{array}$ & $\begin{array}{l}5.2 \\
0.9\end{array}$ & & & & & \\
\hline Donowitz et al. ${ }^{25}$ & $1979-1980$ & & & & 18 & & \\
\hline Wenzel et al. ${ }^{19}$ & 1980-1982 & & & 2 & 7 & 8 & 64 \\
\hline Craven et al. ${ }^{5}$ & $1980-1983$ & & & & 3.5 & 61.6 & \\
\hline Brown et al. ${ }^{4}$ & $1981-1983$ & 5.9 & 6.2 & 1.8 & 11.2 & & \\
\hline Nystrom et al. ${ }^{6}$ & 1983-1984 & & & & & 26 & \\
\hline Chandrasekar et al. ${ }^{7}$ & 1984-1985 & & & 6.6 & 13.9 & 35 & 29.8 \\
\hline Schandorf et al. ${ }^{8}$ & 1984-1985 & & & 4.6 & & & \\
\hline
\end{tabular}




\section{Box 51-1}

\section{Definitions for Nosocomial Infection of the Centers for Disease Control and Prevention}

\section{Primary Bloodstream Infection*}

1. Recognized pathogen isolated from blood culture AND pathogen is not related to infection from another site (other than site of an intravascular device)

\section{OR}

2. One of the following: fever $\left(>38^{\circ} \mathrm{C}\right)$, chills, or hypotension AND any of the following:

a. Common skin contaminant isolated from two blood cultures drawn on separate occasions AND organism is not related to infection at another site

b. Common skin contaminant isolated from blood culture from patient with intravascular access device AND physician institutes appropriate antimicrobial therapy AND organism is not related to infection at another site

c. Positive antigen test on blood AND organism is not related to infection at another site

OR

3. Patient $\leq 12$ months of age has one of the following: fever $\left(>38^{\circ} \mathrm{C}\right)$, hypothermia $\left(<37^{\circ} \mathrm{C}\right)$, apnea, or bradycardia AND one of the following:

a. Common skin contaminant isolated from two blood cultures drawn on separate occasions AND organism is not related to infection at another site (other than site of an intravascular device)

b. Common skin contaminant isolated from blood culture from patient with intravascular access device AND physician institutes appropriate antimicrobial therapy AND organism is not related to infection at another site

c. Positive antigen test on blood AND pathogen is not related to infection at another site

Clinically Defined Pneumonia (PNU1)

1. For any patient, two or more serial chest radiographs with one or more of the following: new or progressive and persistent infiltrate, consolidation, cavitation, $\mathrm{AND}$ at least one of the following:

- Fever $\left(>38^{\circ} \mathrm{C}\right.$ or $\left.>100.4^{\circ} \mathrm{F}\right)$ with no other recognized cause

- Leukopenia $\left(<4000 \mathrm{WBC} / \mathrm{mm}^{3}\right)$ or leukocytosis $\left(12,000 \mathrm{WBC} / \mathrm{mm}^{3}\right)$

- For adults $>70$ years old, altered mental status with no other recognized cause

AND at least two of the following:

- New onset of purulent sputum or change in character of sputum, increased respiratory secretions, or increased suctioning requirements

- New onset of worsening cough, dyspnea, or tachypnea

- Rales or bronchial breath sounds
- Worsening gas exchange (e.g., $\mathrm{O}_{2}$ desaturation [e.g., $\left.\mathrm{PaO}_{2} / \mathrm{FIO}_{2} \leq 240\right]$ ), increased oxygen requirements, or increased ventilation demands

OR

2 . For infant $\leq 1$ year old, two or more serial chest radiographs with one or more of the following: new or progressive and persistent infiltrate, consolidation, cavitation, or pneumatocele AND worsening gas exchange (e.g., $\mathrm{O}_{2}$ desaturation [e.g., $\left.\mathrm{PaO}_{2} / \mathrm{FIO}_{2} \leq 240\right]$ ), increased oxygen requirements, or increased ventilation demands AND at least three of the following:

- Temperature instability with no other recognized cause

- Leukopenia $\left(<4000 \mathrm{WBC} / \mathrm{mm}^{3}\right)$ or leukocytosis $\left(\geq 12,000 \mathrm{WBC} / \mathrm{mm}^{3}\right)$ and left shift $(\geq 10 \%$ band forms)

- New onset of purulent sputum, change in character of sputum, increased respiratory secretions, or increased suctioning requirements

- Apnea, tachypnea, nasal flaring with retraction of chest wall, or grunting

- New onset of worsening cough, dyspnea, or tachypnea

- Wheezing, rales, or rhonchi

- Cough

- Bradycardia ( $<100$ beats/min) or tachycardia $(>170$ beats/min)

\section{OR}

3. Alternate criteria for child $>1$ OR $<12$ years old, two or more serial chest radiographs with one or more of the following: new or progressive and persistent infiltrate, consolidation, cavitation, AND at least three of the following:

- Fever $\left(>38^{\circ} \mathrm{C}\right.$ or $\left.>100.4^{\circ} \mathrm{F}\right)$ with no other recognized cause

- Leukopenia $\left(<4000 \mathrm{WBC} / \mathrm{mm}^{3}\right)$ or leukocytosis $\left(\geq 12,000 \mathrm{WBC} / \mathrm{mm}^{3}\right)$

- New onset of purulent sputum, change in character of sputum, increased respiratory secretions, or increased suctioning requirements

- New onset of worsening cough, dyspnea, or tachypnea

- Rales or bronchial breath sounds

- Worsening gas exchange (e.g., $\mathrm{O}_{2}$ desaturation [e.g., $\left.\mathrm{PaO}_{2} / \mathrm{FIO}_{2} \leq 240\right]$ ), increased oxygen requirements, or increased ventilation demands

\section{Laboratory-Defined Pneumonia (PNU2)}

1. Two or more serial chest radiographs with one or more of the following: new or progressive and persistent infiltrate, consolidation or cavitation, AND at least one of the following: 


\section{Box 51-1}

\section{Definitions for Nosocomial Infection of the Centers for Disease Control and Prevention-cont'd}

- Fever $\left(>38^{\circ} \mathrm{C}\right.$ or $\left.>100.4^{\circ} \mathrm{F}\right)$ with no other recognized cause

- Leukopenia $\left(<4000 \mathrm{WBC} / \mathrm{mm}^{3}\right)$ or leukocytosis $\left(\geq 12,000 \mathrm{WBC} / \mathrm{mm}^{3}\right)$

- For adults $\geq 70$ years old, altered mental status with no other recognized cause

AND at least one of the following:

- New onset of purulent sputum, change in character of sputum, increased respiratory secretions, or increased suctioning requirements

- New onset of worsening cough, dyspnea, or tachypnea

- Rales or bronchial breath sounds

- Worsening gas exchange (e.g., $\mathrm{O}_{2}$ desaturation [e.g., $\left.\mathrm{PaO}_{2} / \mathrm{FIO}_{2} \leq 240\right]$ ), increased oxygen requirements, or increased ventilation demands

AND at least one of the following:

- Positive growth in blood culture not related to another source of infection

- Positive growth in culture of pleural fluid

- Positive quantitative culture from minimally contaminated lower respiratory tract specimen (e.g., bronchoalveolar lavage or protected specimen brushing)

- $\geq 5 \%$ bronchoalveolar lavage-obtained cells contain intracellular bacteria on direct microscopic examination (e.g., Gram stain)

- Histopathologic examination shows at least one of the following evidences of pneumonia:

Abscess formation or foci of consolidation with intense neutrophil accumulation in bronchioles and alveoli

Positive quantitative culture of lung parenchyma

Evidence of lung parenchyma invasion by fungal hyphae or pseudohyphae

\section{Asymptomatic Urinary Tract Infection}

1. An indwelling urinary catheter is present within 7 days before urine is cultured AND patient has no fever $\left(>38^{\circ} \mathrm{C}\right)$, urgency, frequency, dysuria, or suprapubic tenderness AND has urine culture of $\geq 10^{5}$ organ- isms $/ \mathrm{mL}$ urine with no more than two species or organisms

\section{OR}

2. No indwelling urinary catheter is present within 7 days before the first of two urine cultures with $>10^{5}$ organisms $/ \mathrm{mL}$ urine of the same organism with no more than two species of organisms AND patient has no fever $\left(>38^{\circ} \mathrm{C}\right)$, urgency, frequency, dysuria, or suprapubic tenderness

\section{Sinusitis}

1. Organism isolated from culture of purulent material obtained from sinus cavity

OR

2. One of the following: fever $\left(>38^{\circ} \mathrm{C}\right)$, pain or tenderness over the involved sinus, headache, purulent exudate, or nasal obstruction AND either of the following:

a. Positive transillumination

b. Radiographic evidence of infection

\section{Gastroenteritis}

1. Acute onset of diarrhea (liquid stools for $>12$ hours) with or without vomiting or fever $\left(>38^{\circ} \mathrm{C}\right)$ AND no likely noninfectious cause (e.g., diagnostic tests, therapeutic regimen, acute exacerbation of a chronic condition, psychologic stress)

\section{OR}

2. Two of the following with no other recognized cause: nausea, vomiting, abdominal pain, or headache AND any of the following:

a. Enteric pathogen isolated from stool culture or rectal swab

b. Enteric pathogen detected by routine or electron microscopy examination

c. Enteric pathogen detected by antigen or antibody assay on feces or blood

d. Evidence of enteric pathogen detected by cytopathic changes in tissue culture (toxin assay)

e. Diagnostic single antibody titer (IgM) or fourfold increase in paired serum samples (IgG) for pathogen

*All intravascular device-related bloodstream infections are classified with primary bloodstream infections.

From Horan TC, Gaynes RP: Surveillance of nosocomial infections. In Mayhall CG (ed): Hospital Epidemiology and Infection Control, 3rd ed. Philadelphia, Lippincott Williams \& Wilkins, 2004, pp 1659-1702.

The microbial profile of infections at individual sites in ICU patients is shown in Table 51-3. There has been an unrelenting increase in nosocomial infections caused by intrinsically resistant organisms during the past decade, especially coagulase-negative staphylococci, S. aureus, enterococci, $P$. aeruginosa and other resistant gramnegative bacilli, and Candida. ${ }^{32,35,42,43}$ Moreover, the incidence of infection caused by organisms with acquired resistance, especially methicillin-resistant $S$. aureus (MRSA); enterococci resistant to vancomycin (VRE), ampicillin, or both drugs; and gram-negative bacilli resistant to extended-spectrum beta-lactams and fluoroquinolones, has increased even more sharply (Fig. 51-2) ${ }^{44}$

Nosocomial infections acquired in the ICU clearly differ from infections acquired in non-ICU patient care units within the same institutions. Overall rates 

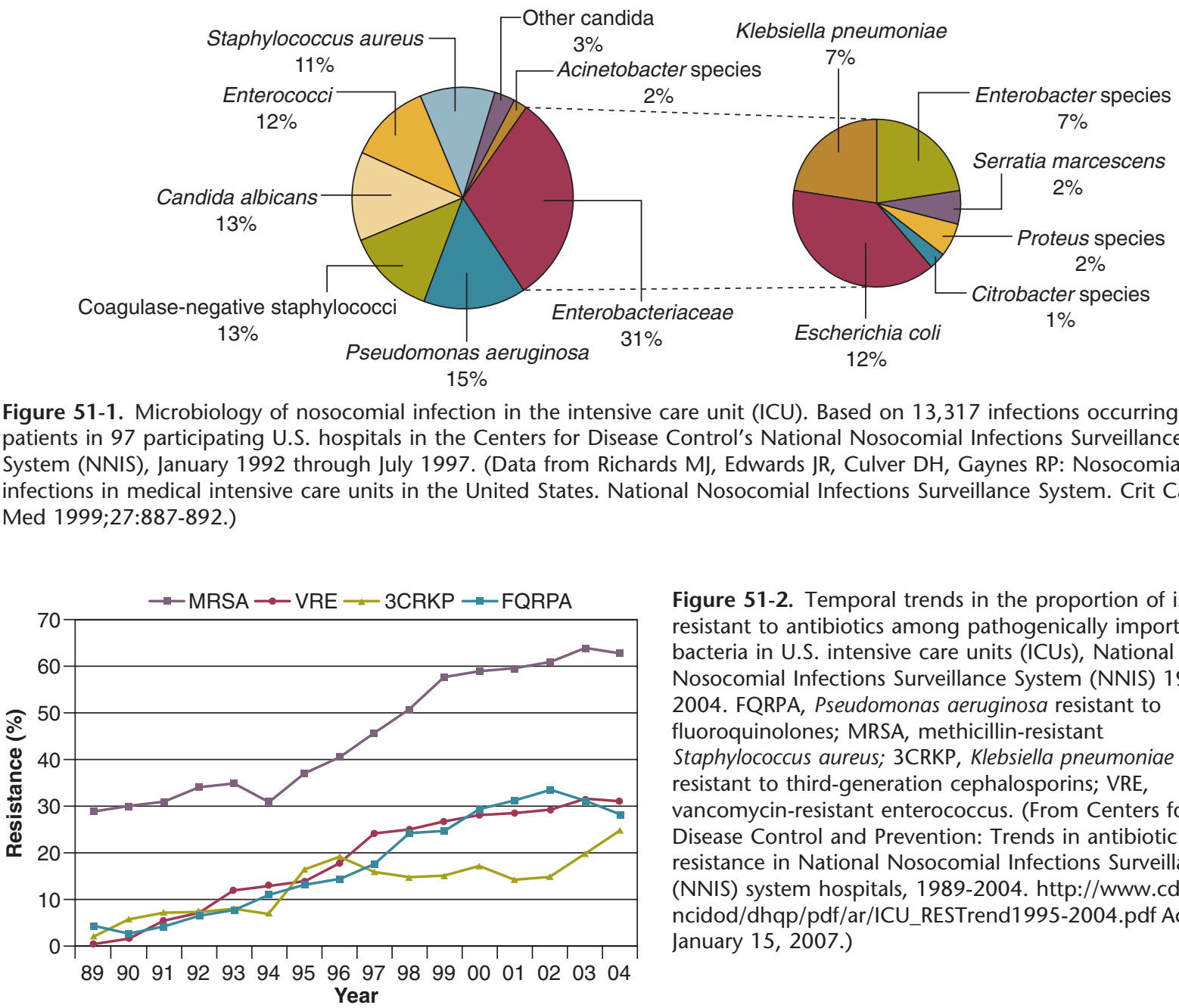

Figure 51-2. Temporal trends in the proportion of isolates resistant to antibiotics among pathogenically important bacteria in U.S. intensive care units (ICUs), National Nosocomial Infections Surveillance System (NNIS) 19892004. FQRPA, Pseudomonas aeruginosa resistant to fluoroquinolones; MRSA, methicillin-resistant Staphylococcus aureus; 3CRKP, Klebsiella pneumoniae resistant to third-generation cephalosporins; VRE, vancomycin-resistant enterococcus. (From Centers for Disease Control and Prevention: Trends in antibiotic resistance in National Nosocomial Infections Surveillance (NNIS) system hospitals, 1989-2004. http://www.cdc.gov/ ncidod/dhqp/pdf/ar/ICU_RESTrend1995-2004.pdf Accessed January 15, 2007.)

Table 51-2. Rates of Device-Related Nosocomial Infection in U.S. Hospital ICUs, Expressed per 1000 Device-Days*

\begin{tabular}{|llll|} 
& & Rate (No. of Cases Per 1000 Device-Days) \\
\cline { 2 - 4 } Type of Infection & Type of ICU & Median & 25th to 75th Percentile of Hospitals \\
\hline Catheter-associated urinary tract infection & PICUs & 3.6 & $1.6-6.1$ \\
& MICUs & 4.7 & $2.5-7.1$ \\
& SICUs & 3.8 & $2.3-6.5$ \\
\hline Ventilator-associated pneumonia & PICUs & 2.3 & $0.9-4.8$ \\
& MICUs & 3.7 & $2.1-6.2$ \\
\hline Central line-associated bloodstream infections & SICUs & 8.3 & $4.7-12.2$ \\
\multicolumn{2}{l}{ PICUs } & 5.2 & $3.0-8.1$ \\
\hline *From the nearly 300 hospitals in the Centers for Disease Control and Prevention's National Nosocomial Infections Surveillance System (NNIS) study, \\
1992-2004. \\
ICU, intensive care unit; MICU, medical ICU; PICU, pediatric ICU; SICU, surgical ICU. \\
From National Nosocomial Infections Surveillance (NNIS) System Report: Data Summary from January 1992 through June 2004, issued October \\
2004. Am J Infect Control 2004;32:470-485.
\end{tabular}

are two to three times higher, and rates of ventilatorassociated pneumonia (VAP) and primary bacteremiamost of which originate from intravascular devices-are 10 times higher. A far greater proportion of ICU-acquired infections are caused by antibiotic-resistant bacteria because the intensive antimicrobial therapy characteristic of modern-day ICUs grossly distorts patients' microflora. Moreover, more than half of all nosocomial epidemics now occur among the $10 \%$ of hospitalized patients confined to an ICU. ${ }^{19,32}$ Finally, the risk of occupationally 
Table 51-3. Profile of Nosocomial Infection in the ICU

\begin{tabular}{|c|c|c|}
\hline Infection & Major Pathogen & Risk Factors \\
\hline Urinary tract & $\begin{array}{l}\text { Pseudomonas aeruginosa } \\
\text { Klebsiella and Enterobacter spp. } \\
\text { Enterococci } \\
\text { Staphylococcus epidermidis } \\
\text { Candida spp. }\end{array}$ & $\begin{array}{l}\text { Urinary catheter } \\
\text { Monitoring of urine output } \\
\text { Other urologic manipulation or } \\
\text { bladder irrigations } \\
\text { Renal transplantation } \\
\text { Diabetes } \\
\text { Female }>\text { male }\end{array}$ \\
\hline Pneumonia & $\begin{array}{l}\text { P. aeruginosa } \\
\text { Klebsiella and Enterobacter spp. } \\
\text { Serratia marcescens } \\
\text { Acinetobacter spp. } \\
\text { Staphylococcus aureus } \\
\text { Oral anaerobes } \\
\text { Immunosuppression }\end{array}$ & $\begin{array}{l}\text { Tracheostomy } \\
\text { Endotracheal tube, reintubation } \\
\text { Nasogastric tube } \\
\text { Intracranial pressure monitoring } \\
\text { Stress ulcer prophylaxis with } \mathrm{H}_{2} \\
\quad \text { blocker or antacids } \\
\text { Immunosuppression } \\
\text { Granulocytopenia }\end{array}$ \\
\hline Postsurgical wound & $\begin{array}{l}\text { Staphylococcus aureus } \\
\text { Escherichia coli and other gram-negative bacilli } \\
\text { Enterococci } \\
\text { Bacteroides fragilis and other bowel anaerobes }\end{array}$ & $\begin{array}{l}\text { Trauma, especially penetrating } \\
\text { abdominal injury } \\
\text { Gastrointestinal or radical } \\
\text { gynecologic surgery } \\
\text { Prolonged operation } \\
\text { Immunosuppressive therapy } \\
\text { Granulocytopenia } \\
\text { Hepatic transplantation } \\
\text { Central venous catheter in place } \\
>5 \text { days }\end{array}$ \\
\hline $\begin{array}{l}\text { Bacteremia from intravascular devices } \\
\text { Catheter related }\end{array}$ & $\begin{array}{l}\text { Coagulase-negative staphylococci } \\
\text { S. aureus } \\
\text { Candida spp. }\end{array}$ & $\begin{array}{l}\text { Heavy colonization of insertion } \\
\text { site skin } \\
\text { Femoral vein insertions } \\
\text { Catheter guidewire exchanges }\end{array}$ \\
\hline Contaminated infusate & $\begin{array}{l}\text { Enterobacter spp. } \\
\text { S. marcescens } \\
\text { Citrobacter spp. } \\
\text { Pseudomonas cepacia or Xanthomonas } \\
\quad \text { maltophilia }\end{array}$ & \\
\hline Antibiotic-associated diarrhea or colitis & Clostridium difficile & $\begin{array}{l}\text { Prolonged antibiotic therapy, } \\
\text { especially with clindamycin or } \\
\text { broad-spectrum } \beta \text {-lactams } \\
\text { Enteral tube feeding }\end{array}$ \\
\hline Candidemia & Candida spp. & $\begin{array}{l}\text { Broad-spectrum, prolonged } \\
\text { antimicrobial therapy } \\
\text { Mucosal or urinary colonization } \\
\text { Central venous catheter } \\
\text { Hyperalimentation } \\
\text { Renal failure }\end{array}$ \\
\hline
\end{tabular}

acquired infection among health care workers (HCWs), particularly by bloodborne viruses and herpes simplex virus (HSV), is highest among ICU personnel, as contrasted with those who work in non-ICU patient care units (see Protection of Health Care Workers in the Intensive Care Unit later).

\section{MORBIDITY AND ECONOMIC IMPACT}

Nosocomial infections have a considerable impact on morbidity and mortality and are estimated to affect more than 2 million patients in U.S. hospitals annually.$^{45}$ Table 51-4 summarizes major studies that have examined mortality, length of stay, and costs associated with the major nosocomial infections in U.S. hospitals. ${ }^{2-18}$ Nosocomial infections have been ascribed by the National Institute of Medicine to be responsible for more than 80,000 hospital deaths each year and in 1995 resulted in more than $\$ 5$ billion in excess health care costs. ${ }^{45}$ Considering that nosocomial infections acquired by ICU patients account for nearly half of all infections in most hospitals, progress in reducing the incidence of infection acquired within ICUs could produce substantial economic benefits. 


\begin{tabular}{|c|c|c|c|c|c|}
\hline \multirow[b]{2}{*}{ Infection } & \multirow[b]{2}{*}{ Description } & \multirow{2}{*}{$\begin{array}{l}\text { Average Extra } \\
\text { Days in } \\
\text { Hospital or ICU } \\
\text { Per Infection }\end{array}$} & \multirow{2}{*}{$\begin{array}{l}\text { Average Extra } \\
\text { Charges or } \\
\text { Costs per } \\
\text { Infection }(\$)\end{array}$} & \multicolumn{2}{|c|}{ Excess Mortality } \\
\hline & & & & Unadjusted & Attributable \\
\hline \multicolumn{6}{|c|}{ Postoperative Surgical Wound Infection } \\
\hline Kirkland et al, $1999^{600}$ & $\begin{array}{l}\text { CABG, vascular surgery, } \\
\text { abdominal surgery, } \\
\text { orthopedic surgery }\end{array}$ & 6.5 & 3,089 & NR & $4.3 \%$ \\
\hline Whitehouse et al, $2002^{601}$ & Orthopedic surgery & 14 & 17,708 & NR & $0.0 \%$ \\
\hline Hollenbeak et al, $2000^{602}$ & $\begin{array}{l}\text { Deep chest infection } \\
\text { following } C A B G\end{array}$ & 20 & 20,012 & NR & $19.4 \%$ \\
\hline McGarry et al, $2004^{603}$ & $\begin{array}{l}\text { All major surgical procedures; } \\
\text { only S. aureus infections } \\
\text { included }\end{array}$ & 13 & 53,625 & NR & $16.8 \%$ \\
\hline Herwaldt et al, $2006^{604}$ & All major surgical procedures & NR & 3,021 & $1.2 \%$ & $0 \%$ \\
\hline \multicolumn{6}{|c|}{ Ventilator-Associated Pneumonia } \\
\hline Fagon et al, $1993^{605}$ & Medical and surgical patients & 13 & NR & NR & $27.1 \%$ \\
\hline Heyland et al, $1999^{606}$ & Medical and surgical patients & 4.3 & NR & NR & $5.8 \%$ \\
\hline Bercault et al, $2001^{607}$ & Medical and surgical patients & 5 & NR & NR & $27.4 \%$ \\
\hline Rello et al, $2002^{608}$ & Medical and surgical patients & 11 & 40,000 & NR & $0 \%$ \\
\hline Warren et al, $2003^{609}$ & Medical and surgical patients & 25 & 11,897 & $16 \%$ & NR \\
\hline Cocanour et al, $2005^{610}$ & Trauma patients & 15 & 57,158 & NR & $0 \%$ \\
\hline \multicolumn{6}{|l|}{ Bloodstream Infection } \\
\hline Pittet et al, $1994^{264}$ & Surgical ICU & 24 & 40,000 & NR & $35 \%$ \\
\hline Digiovine et al, $1999^{265}$ & Adult ICU & 10 & 34,508 & NR & $4 \%$ \\
\hline Slonim et al, $2001^{611}$ & Pediatric ICU & 22 & 35,000 & NR & $13 \%$ \\
\hline Warren et al, $2006^{612}$ & Adults & 7.5 & 11,971 & $23 \%$ & NR \\
\hline \multicolumn{6}{|c|}{ Catheter-Associated Urinary Tract Infection } \\
\hline Bryan et al, $1984^{469}$ & Medical and surgical patients & NR & NR & NR & $12 \%$ \\
\hline Tambyah et al, $2002^{472}$ & Medical and surgical patients & NR & 589 & NR & NR \\
\hline
\end{tabular}

\section{PATHOGENESIS AND EPIDEMIOLOGY}

\section{Pathogenesis}

The occurrence of nosocomial infection reflects the conjunction in space and time of a pathogenic microbe and a vulnerable patient, catalyzed by events associated with hospitalization and the patient's care. Many patients admitted to an ICU are intrinsically more susceptible to infection because of underlying diseases or conditions associated with impaired immunity such as cancer, trauma ${ }^{46}$ or advanced age ${ }^{47}$ or because of immunosuppression associated with malnutrition ${ }^{48}$ or therapy with corticosteroids, ${ }^{49}$ cancer chemotherapeutic agents, ${ }^{50}$ or other immunosuppressive drugs. ${ }^{51}$ Moreover, many drugs have indirect effects that increase susceptibility to infection, such as narcotics or sedatives that impair the capacity to protect the airway, or antacids or $\mathrm{H}_{2}$-histamine receptor antagonists that neutralize gastric acidity, producing gastric overgrowth by gram-negative bacilli, ${ }^{52}$ increasing the risk of nosocomial pneumonia. ${ }^{53}$ Even transfusion therapy produces immunosuppression and increases the risk of nosocomial infection. ${ }^{54}$

Moreover, most nosocomial pathogens exhibit resistance to antibiotics (see Figs. 51-1 and 51-2),,$^{42,43,55-58}$ and many are also more virulent because of (1) their capacity to subsist or even multiply in aqueous reservoirs for prolonged periods (e.g., pseudomonads ${ }^{59}$ or Legionella pneumoph$\left.i l a^{60}\right)$; (2) the elaboration of endotoxins (e.g., all of the gramnegative bacilli) or exotoxins (P. aeruginosa, ${ }^{61} \mathrm{C}$. difficile, ${ }^{62}$ or S. aureus ${ }^{63}$ ); or (3) the production of adhesions ${ }^{64}$ or exoglycocalyx ${ }^{65}$ (e.g., coagulase-negative staphylococci), conferring the capacity to adhere avidly and form biofilms on biologic and prosthetic surfaces resistant to host defenses ${ }^{66}$ and even antibiotics. ${ }^{67}$ Because most patients in ICUs receive broad-spectrum antibiotics, resistant nosocomial organisms have an enormous ecologic advantage and, in Darwinian fashion, predictably supplant the normal cutaneous, respiratory, and gastrointestinal flora. 
In most cases, colonization is the first step in the progression to nosocomial infection, ${ }^{68}$ especially if the patient is already vulnerable because of underlying disease, if the organism is more virulent or resistant to antibiotics, or if the patient has invasive medical devices that assist invasion by colonizing organisms, bypassing or further impairing host defenses.

\section{Reservoirs and Transmission}

The epidemiology of an infection consists of the reservoirs and mode or modes of transmission of the pathogen or pathogens and those factors associated with an increased (or decreased) risk of infection. Understanding the epidemiology of an infection is essential to developing effective strategies for its prevention.

In the ICU the major reservoir of nosocomial organisms is the infected or colonized patient (Fig. 51-3). ${ }^{28}$ Whereas Streptococcus pneumoniae, ${ }^{69}$ Mycobacterium tuberculo- sis, ${ }^{70-72}$ Legionella, ${ }^{41}$ Aspergillus and Zygomycetes, ${ }^{36-38}$ measles, ${ }^{73}$ rubella, ${ }^{74}$ and influenza $\mathrm{A}^{75}$ are transmitted by the airborne route, the best evidence suggests that most aerobic bacteria-particularly $S$. aureus, ${ }^{76}$ enterococci $^{29}$ and the enteric gram-negative bacilli ${ }^{77}$; many viruses such as hepatitis $\mathrm{A}, \mathrm{RSV},{ }^{78}$ and rotaviruses ${ }^{79} ; C$. difficile $^{80}$; and even Candida ${ }^{81}$-are spread in the ICU on the hands of medical personnel, who themselves are not infected or even permanently colonized. Surgery and exposure to invasive devices of all types greatly amplify transmission, colonization, and susceptibility to infection. $^{28,82}$

Outbreaks of S. aureus ${ }^{83}$ or group A streptococcal infection $^{84}$ usually indicate a health care provider who is a carrier of the epidemic strain. Airborne spread of gramnegative bacilli is probably rare unless unusual environmental circumstances generate massively contaminated aerosols. $^{85}$

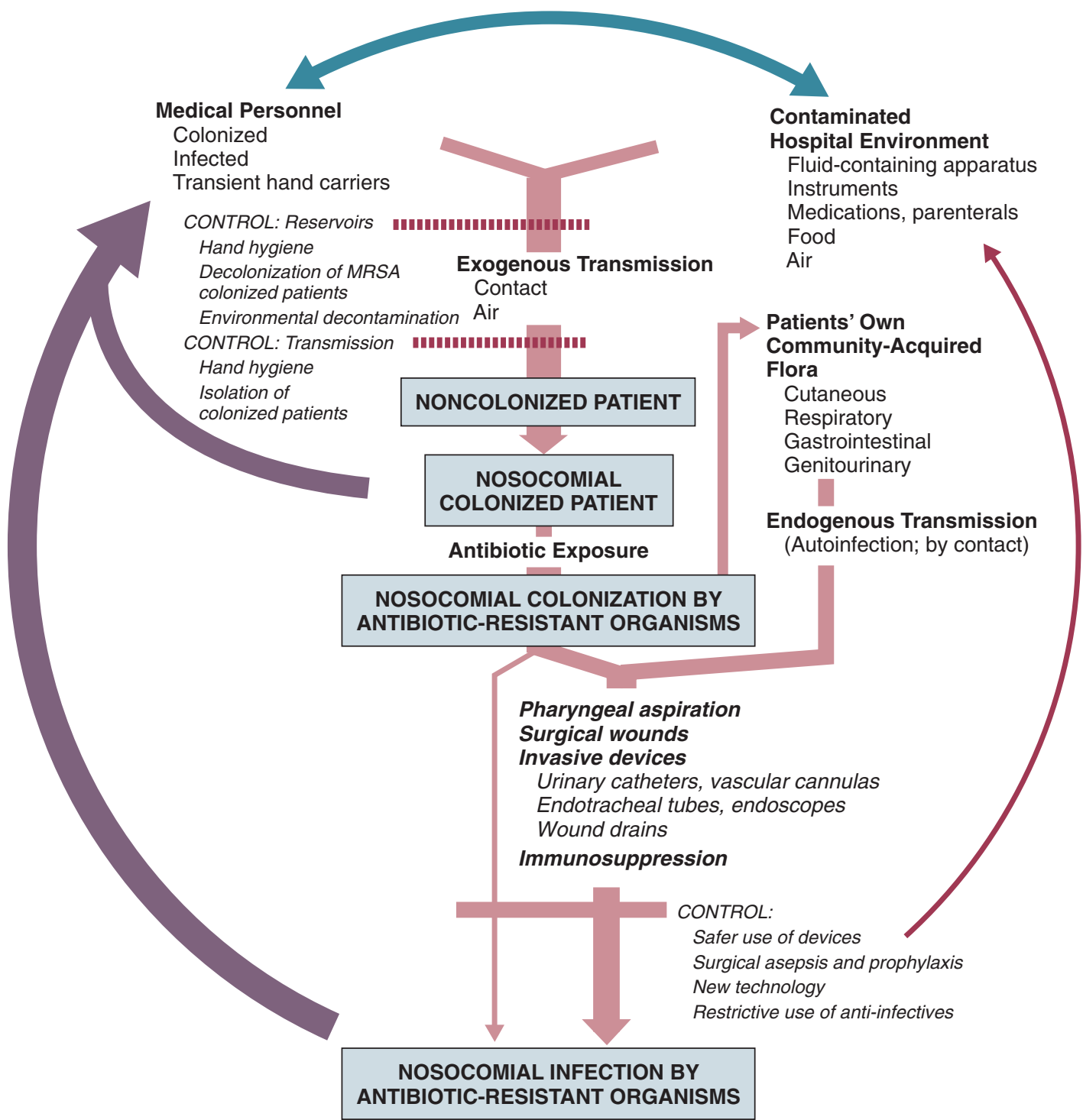

Figure 51-3. The epidemiology of nosocomial infection. Transmission occurs mainly by contact spread and, to a much lesser extent, the airborne route. Aspiration, surgical wounds, and exposure to invasive medical devices enormously amplify transmission, colonization, and susceptibility to infection. (From Maki DG: Control of colonization and transmission of pathogenic bacteria in the hospital. Ann Intern Med 1978;89[Suppl]:777-780.) 
Increasing evidence suggests that many nosocomial infections acquired in the ICU derive from resistant organisms of enteric origin ${ }^{86-89}$ or present on $\operatorname{skin}^{86,87}$ or in the lower respiratory tract ${ }^{88}$ on admission to the ICU. This explains the failure of conventional infection control practices, based on the use of barriers, to prevent extrinsically acquired infection. ${ }^{90}$ Whereas food ${ }^{91}$ and even enteral feeding preparations ${ }^{92}$ are often heavily contaminated by microorganisms, studies have not conclusively linked such contamination to disease.

Nosocomial organisms originating from colonized or infected patients are readily perpetuated and spread in contaminated medical apparatus or devices ${ }^{28}$ such as urine-collection receptacles, ${ }^{93}$ respiratory therapy equipment, ${ }^{94,95}$ transducers used for hemodynamic monitoring, ${ }^{96}$ dialysis machines, ${ }^{97,98}$ and fiberoptic bronchoscopes and endoscopes. ${ }^{95,99-101}$ Given the implicit close proximity of vulnerable ICU patients and the HCWs who have repeated contact with them each day, it is almost predictable that the ICU is a milieu within the hospital uniquely conducive to the epidemic infection, especially infections caused by antibiotic-resistant pathogens.

Although successful immune enhancement could in theory create a protective final barrier against infection by nosocomial organisms, the unique features of nosocomial infection-enormous microbial heterogeneity, pervasive effects of invasive devices and procedures, and often large infecting inocula-can overwhelm the healthiest patient's immune defenses. Measures to eradicate reservoirs of nosocomial pathogens and to block transmission, based on a thorough understanding of epidemiology, seem much more likely to be protective at present.

\section{Risk Factors}

Risk factor analysis using powerful statistical techniques of multivariable analysis can identify the circumstances that put a patient at increased risk for nosocomial infection and further guide the development of preventive strategies. Risk factors based on prospectively collected data and, in most cases, the use of multivariable analysis are listed in Table 51-3 for urinary tract infection, ${ }^{102,103}$ pneumonia, ${ }^{104,105}$ postoperative surgical infection, ${ }^{106}$ intravascular device-related bloodstream infection, ${ }^{107}$ ventriculostomy-associated meningitis, ${ }^{108}$ antibioticassociated colitis, ${ }^{34,109}$ and deep Candida infection. ${ }^{110-112}$

Critical care medicine is synonymous with cutting-edge, high-tech medicine; mechanical ventilatory support; hemodynamic monitoring; total parenteral nutrition; hemodialysis; intracranial pressure monitoring; innovative forms of surgery; and a huge arsenal of drugs, especially antiinfectives of every genre. This technology, more than anything else, has forced critical care medicine to accept the necessity for nosocomial infection control. In general, invasive devices of all types are far more important in determining susceptibility to nosocomial infection than underlying diseases (see Tables 51-3 and 51-5). However, this should be viewed as welcome news: There is far more hope for reducing nosocomial infections in the coming decade by innovative improvements in aseptic technique
Table 51-5. Significant Risk Factors for Nosocomial Infection in the ICU as Determined by Multivariate Analysis of Prospectively Collected Databases

\begin{tabular}{|c|c|c|}
\hline $\begin{array}{l}\text { Type of ICU } \\
\text { (Investigators) }\end{array}$ & Risk Factors & $\begin{array}{l}\text { Approximate } \\
\text { Magnitude of } \\
\text { Increased Risk }\end{array}$ \\
\hline Pediatric ICU ${ }^{23,613}$ & $\begin{array}{l}\text { Patent ductus arteriosus } \\
\text { Low birth weight } \\
\text { Endotracheal tube } \\
\text { Hyperalimentation } \\
\text { Surgery } \\
\text { High fraction inspired } \\
\text { oxygen } \\
\text { Umbilical catheter } \\
\text { Blood product therapy } \\
\text { Central venous catheter } \\
\text { Mechanical ventilatory } \\
\text { support }\end{array}$ & $\begin{array}{l}28.2 \\
\overline{7}^{\dagger} \\
5.9 \\
- \\
- \\
- \\
- \\
-\end{array}$ \\
\hline $\begin{array}{l}\text { Adult medical } \\
\text { and surgical } \\
\text { ICUs }^{5}\end{array}$ & $\begin{array}{l}\text { Urinary catheter } \\
>10 \text { days } \\
\text { ICU confinement } \\
>3 \text { days } \\
\text { Intracranial pressure } \\
\text { monitor } \\
\text { Arterial line } \\
\text { Shock }\end{array}$ & $\begin{array}{l}3.2 \\
2.5 \\
2.5 \\
1.5 \\
2.5\end{array}$ \\
\hline
\end{tabular}

and advances in the technology of invasive devices than by breakthroughs that will reverse the ravages of chronic organ failure or degenerative diseases such as type 1 diabetes mellitus.

\section{GENERAL CONTROL MEASURES}

\section{Hospital Infection Control Programs}

Beginning in the late 1960s, scattered U.S. hospitals began to establish infection control programs to conduct surveillance, to develop infection control policies, and especially to try to implement control measures more consistently. ${ }^{113}$ In 1976 JCAHO added to its requirements for hospital accreditation the establishment of a formal infection control program.

In the early 1970s the CDC undertook determining the effectiveness of nosocomial infection surveillance and control programs in the United States through the auspices of the Study of the Efficacy of Nosocomial Infection Control (SENIC). The goals of SENIC were to determine the extent to which infection control programs had been adopted by U.S. hospitals and to ascertain how much these programs had reduced rates of nosocomial infection. SENIC was launched by a survey of all U.S. hospitals to determine the characteristics of infection control programs and was completed in $1975-1976$ by a review of more than 339,000 patient medical records in 338 randomly selected hospitals. ${ }^{114}$

The SENIC found that hospitals reduced their nosocomial infection rates by approximately $32 \%$ if their surveil- 
lance and infection control program included four components: (1) emphasis on both surveillance and an infection control program, (2) at least one full-time infection control practitioner for every 250 beds, (3) a trained hospital epidemiologist, and (4) surveillance of surgical wound infections with feedback of wound infection rates to practicing surgeons. ${ }^{115}$ However, the relative importance of each component varied for the four major types of nosocomial infections (surgical wound infections, urinary tract infections, bloodstream infections, and pneumonia). ${ }^{15,116}$ SENIC suggests that nearly one third of all nosocomial infections are in theory preventable, whereas a 1983 survey of surveillance and control programs in a random sample of U.S. hospitals found that failure to implement all essentials of the program, particularly to have an adequate number of infection control practitioners or a trained hospital epidemiologist or to disseminate wound infection rates to surgeons, was greatly limiting the potential for prevention: U.S. hospitals were estimated to be preventing only $9 \%$ of all infections. ${ }^{117}$

It is hoped that surveillance and control programs will continue to evolve. Prevention of nosocomial infections is a major priority of the U.S. Public Health Service, ${ }^{118}$ JCAHO ${ }^{31}$ and the Institute of Medicine. ${ }^{119}$ With the shift to prospective-payment reimbursement, hospitals now have a powerful financial incentive to reduce their rates of nosocomial infection, ${ }^{120}$ and it can be anticipated that efforts to prevent hospital-acquired infections will assume ever greater importance.

JCAHO now mandates that all hospitals have an active program for surveillance, prevention, and control of hospital-acquired infections, which begins with an institutional infection control committee with representation from the major clinical services and hospital departments including the institution's ICUs. The most essential members of the infection control program are the infection control practitioner(s), usually registered nurse(s), and the hospital epidemiologist, usually a physician with training in infectious diseases or microbiology, who implement the policies developed by the committee, educate hospital personnel about nosocomial infection control, and investigate suspected outbreaks (Box 51-2).

Surveillance of nosocomial infections is the cornerstone of an effective infection control program and offers numerous potential benefits ${ }^{116,121}$ : (1) It permits determination of baseline (expected) infection rates, assisting recognition of outbreaks and evaluation of new policies and control measures; (2) it identifies institutional problems that require attention, permitting focused infection control efforts and education; (3) it provides reliable data that can be disseminated to individual departments, increasing awareness and involvement of individual staff members; (4) it increases the visibility of the infection control staff on patient care units, providing an opportunity for consultation and ad hoc education; and (5) it facilitates the earliest discovery of patients with communicable infections, permitting timely institution of isolation precautions to limit spread. Because total surveillance (of all infections) is labor intensive, most hospitals now focus

\section{Box 51-2}

\section{Facets of a Hospital Infection Control Program}

- Active infection control committee, with representation from major departments and services including the intensive care units (ICUs)

- Surveillance of nosocomial infections, especially in each ICU

- Comprehensive and regularly updated institutional policies and procedures for prevention of nosocomial infection:

- Surveillance of nosocomial infections

- Isolation and universal precautions

- Sterilization and disinfection

- Indications for and management of invasive procedures and devices

- All types of intravascular catheters

- Hemodynamic monitoring

- Tracheostomy and endotracheal intubation

- Mechanical ventilation and other respiratory therapy

- Bronchoscopy and gastrointestinal endoscopy

- Anesthesia and the operating room

- Hemodialysis

- Intra-aortic balloon pumps

- Cardiopulmonary bypass

- Intracranial pressure monitoring

- Antimicrobial stewardship program

- Guideline for investigation of an epidemic

- Strong liaison with clinical microbiology laboratory

- Representation on the Infection Control Committee

- Laboratory-based surveillance

- Monitoring and reporting of trends in antimicrobial susceptibility

- Retaining important isolates

- Microbiologic support of all infection control activities

- Subtyping of isolates for investigations or studies

- Educational programs for new employees, periodic updates dealing with nosocomial infection control

- Active employee health department:

- Free immunizations (hepatitis B, measles, mumps, rubella, varicella, pertussis, influenza A)

- Tuberculin screening

- Postexposure protocols

- Quality assurance review of implementation of infection control policies and practices

Modified from Maki DG: Nosocomial infection. In Parrillo JE (ed): Current Therapy in Critical Care Medicine, 2nd ed.

Philadelphia, BC Decker, 1991.

their surveillance efforts on infections that are associated with high morbidity (e.g., nosocomial pneumonia), that greatly increase health care costs (e.g., postcardiac surgery sternotomy infections), that are caused by antibioticresistant organisms with potential for spread (e.g., MRSA, 
C. difficile), or that are highly preventable (e.g., intravascular device-related bloodstream infections). ${ }^{116,122}$

The 1990s were characterized by major efforts by hospitals to apply to the numerous facets of health care principles of quality improvement developed by industry. Hospital infection control programs have been working on quality improvement for 20 years ${ }^{123}$ but, influenced by JCAHO, were probably too heavily focused on process, namely, policies and procedures, rather than documenting outcome vis-à-vis reduced infection rates. Infection control programs in most U.S. hospitals are now closely allied with their institutional quality improvement departments. ${ }^{123,124}$

Hospital infection control programs are also regulated by the Occupational Safety and Health Administration (OSHA) in terms of institutional standards and programs to protect HCWs from bloodborne pathogens ${ }^{125}$ and tuberculosis ${ }^{126}$; the Environmental Protection Agency ${ }^{127}$ has also published regulations in terms of disposal and tracking of medical waste-only a small fraction of which is truly biohazardous. ${ }^{128}$

Finally, it is essential that all health care personnel working in an ICU receive training in the epidemiology and control of nosocomial infections. This may be most important for house officers in teaching hospitals, who commonly enter the ICU with only the most rudimentary knowledge of asepsis but have hands-on contact with numerous patients each day. ICU physicians and nurses must be especially familiar with their hospitals' guidelines for the management of invasive devices, particularly intravascular catheters of all types, ${ }^{129}$ urinary catheters, ${ }^{130}$ endotracheal tubes, ${ }^{131}$ and tracheostomies. ${ }^{131}$ Moreover, all physicians need to be made aware that broad-spectrum antimicrobial therapy greatly increases the risks of superinfection by antibiotic-resistant bacteria and Candida, as well as antibiotic-associated colitis caused by $C$. difficile.

\section{Role of the Microbiology Laboratory}

Accurate and timely diagnostic microbiology is as essential for nosocomial infection control as it is for the clinical management of patients' infections. Although many infections can be diagnosed on the basis of clinical criteria alone, cultures and other laboratory tests allow infections to be diagnosed with much greater certainty, and certain infections such as bacteriuria, bacteremia, and fungal and viral infections cannot be diagnosed without cultures or other laboratory tests (see Box 51-1). ${ }^{16}$ Moreover, accurate antimicrobial susceptibility testing of clinical isolates is the only means of monitoring trends in antibiotic resistance of hospital organisms. ${ }^{132,133}$ Most importantly, identifying the microbial cause of nosocomial infections allows epidemiologic tracking of individual pathogens within the hospital, especially those that are commonly spread from patient to patient such as $S$. aureus, beta-hemolytic streptococci, enterococci, and the numerous gram-negative bacilli.

From an organizational standpoint, the institutional infection control program and clinical microbiology laboratory must have a close working relationship (see Box 51-2) to assist surveillance, which must be strongly labora- tory based, ${ }^{116,134}$ and to permit the detection and resolution of potential problems. The laboratory director or a senior member of the laboratory staff should be a permanent member of the infection control committee.

The primary role of the clinical microbiology laboratory in any infection control program is to provide up-to-date clinical microbiologic data for use in the surveillance of nosocomial infections and identification of potential outbreaks. ${ }^{134}$ Protocols should be developed to ensure that laboratory staff immediately contact infection control personnel after the isolation of certain important pathogens such as MRSA or vancomycin-resistant enterococci (VRE) or the appearance of new resistance patterns in endemic organisms such as resistance of Klebsiella species to thirdgeneration cephalosporins or $P$. aeruginosa to aminoglycosides, fluoroquinolones, and carbapenems. Sifting through these data can be time consuming, and developing electronic information systems that streamline this process is essential to improving the efficiency of the infection control program. Commercial software programs that can automate this process are now available. Many of these programs automatically collate microbiologic data, provide rudimentary geographic information, and perform basic statistical analyses that can assist in the surveillance of nosocomial infections and identification of potential outbreaks. ${ }^{135,136}$

Reporting cumulative summaries of antimicrobial susceptibility data (antibiograms) is another essential responsibility of the clinical microbiology laboratory. ${ }^{137,138}$ When implemented appropriately, the timely dissemination of antibiograms helps guide the choice of empiric antimicrobials, pending the results of clinical cultures, and provides valuable data to help the infection control department monitor institutional antimicrobial resistance trends and identify potential outbreaks. ${ }^{139}$ The Clinical and Laboratory Standards Institute-formerly the National Committee for Clinical Laboratory Standards-recommends that institutional antibiograms be updated at least annually and has recently published standards for their content and format. ${ }^{140}$ Automated electronic systems for collating and disseminating nearly real-time antibiograms along with antibiotic-use decision support exist and, when implemented properly, have been effective in improving antimicrobial utilization within the hospital setting. ${ }^{141,142}$

Monitoring of sterilizers with spore tests, environmental sampling, and advanced microbiologic support for epidemiologic investigations are additional responsibilities expected of most clinical microbiology laboratories, although some university hospital programs have dedicated personnel within their infection control programs who perform these activities. ${ }^{134}$

The clinical microbiology laboratory is a key resource in the investigation of a suspected outbreak. One of the first and foremost actions when a nosocomial outbreak is suspected is to immediately retrieve all available isolates of the putative epidemic pathogen for possible subtyping. ${ }^{143}$ The need to move rapidly becomes apparent when it is realized that most hospital laboratories discard cultures as soon as the isolates have been fully characterized. 
All blood isolates should be routinely saved for at least 1 year. ${ }^{143}$ Laboratory personnel must be requested to save clinical isolates of any unusual organisms that are encountered for the first time or clusters of any organism and to inform infection control personnel of the findings and availability of the isolates.

The rapid evolution of molecular microbiology has revolutionized epidemiologic investigation of nosocomial outbreaks. Molecular-based tests for the rapid diagnosis of bacterial, ${ }^{144}$ viral, ${ }^{75,145}$ and fungal ${ }^{146}$ infections are now routinely available in most hospital-based and reference laboratories. Modern molecular tests can reliably detect minute numbers of organisms, allowing direct testing of clinical samples without the need for culture. In modernday clinical virology, molecular tests based on polymerase chain reaction (PCR) for amplification of the pathogen's DNA or RNA have supplanted tissue cultures and now allow rapid diagnosis of infections that would otherwise often not be identifiable by classic methods.

The availability of molecular subtyping systems has greatly strengthened investigations of outbreaks, as well as research on the epidemiology of nosocomial infections. ${ }^{147,148}$ The antimicrobial susceptibility pattern (antibiogram) or the detailed biochemical profile (biotype) is often useful for the initial epidemiologic subtyping of many bacteria and may be adequate for identifying an epidemic caused by an unusual pathogen. However, if an epidemic organism is a common species such as $S$. aureus, it can be difficult or even impossible to know with certainty that an outbreak derives from a common source using these techniques because they lack sufficient discriminatory power. ${ }^{147}$

The new molecular techniques of subtyping such as plasmid profile typing by agarose gel electrophoresis or the use of restriction endonuclease digests with pulsedfield electrophoresis (Fig. 51-4) (DNA fingerprinting) ${ }^{147}$ are now available in most infection control research laboratories but should be adaptable by many hospital laboratories. Genetic probes promise even more powerful tools for investigating outbreaks, particularly those caused by antibiotic-resistant organisms. ${ }^{144}$

Although molecular-based tests offer several advantages over traditional microbiologic techniques, they are not a panacea. A number of molecular diagnostic assays (e.g., analyte specific reagents [ASRs]) marketed for clinical practice do not require approval by the U.S. Food and Drug Administration. ${ }^{144}$ In the absence of published data on their accuracy and precision, the results of these tests must be interpreted with caution and should always undergo extensive in-house validation before widespread adoption. Moreover, the exquisite sensitivity of many of these tests renders them more susceptible to false-positive results as a consequence of environmental contamination $^{149,150}$ and mandates stringent quality control practices and procedures.

\section{Architectural and Environmental Issues}

The role of the inanimate environment on the transmission of nosocomial infections has been a subject of intense debate for decades. It has been shown that hospital sur-

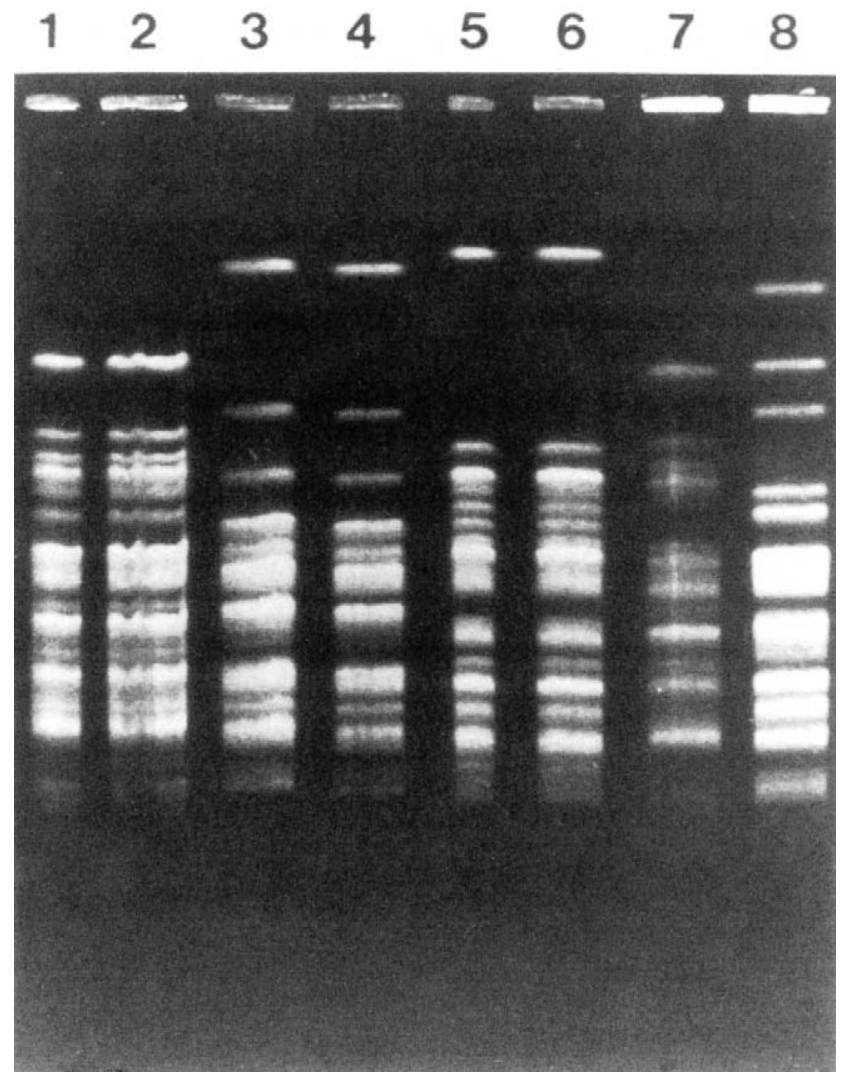

Figure 51-4. Pulse-field electrophoresis patterns of chromosomal DNA of type 10 Pseudomonas aeruginosa. Isolates subjected to Dra1 endonuclease digestion from machines or endoscopes and from patients in three centers using an Olympus automated Endoscope Washer. Lanes 1 and 2, from the University of Wisconsin Hospital and Clinics, 3 and 4 from Center B, and 5 and 6 from Center $C$ also show concordance between each institution's machine or endoscope isolate and patient isolate. Lanes 7 and 8 contain unrelated (control) strains of $P$. aeruginosa. The use of molecular subtyping strongly supported the epidemiologic studies, causally implicating contaminated endoscope washers in the genesis of endoscopy-associated nosocomial $P$. aeruginosa infections in U.S. centers using the flawed washer. (From Alvarado C), Stolz SM, Maki DG: Nosocomial infections from contaminated endoscopes: A flawed automated endoscope washer. An investigation using molecular epidemiology. Am J Med 1991;91:272S-280S.)

faces are almost universally contaminated by potentially pathogenic bacteria such $S$. aureus, ${ }^{151}$ enterococcus, ${ }^{152}$ and gram-negative bacilli such as Acinetobacter baumanii. ${ }^{153}$ Prior to the 1970s, infection control personnel routinely sampled hospital surfaces. Despite this level of surface colonization, early studies found that the inanimate environment-surfaces, walls, and even air-does not contribute materially to the occurrence of most nosocomial infections, ${ }^{154}$ other than invasive infections caused by airborne Aspergillus and other filamentous fungi in seriously immunocompromised patients. ${ }^{36,37}$

Although inanimate surfaces may rarely be involved in the direct transmission of infection to patients, more recent evidence suggests that surfaces may well play an important role in the nosocomial acquisition of patho- 
genic bacteria, indirectly, through contact with $\mathrm{HCWs}$ hands and equipment (see Fig. 51-3). This indirect route of infection is of particular importance in the ICU, where all patients are heavily exposed to invasive devices and have a high risk of infection. In the ICU the inanimate environment may become a reservoir for the transmission of resistant nosocomial organisms such as MRSA, ${ }^{155,156}$ C. difficile, ${ }^{80,157} \mathrm{VRE}^{152,158}$ and gram-negative bacilli such as Klebsiella spp., Acinetobacter spp., and Enterobacter organisms. ${ }^{159,160}$ Studies have shown that enhanced surface decontamination with hypochlorite-containing cleaning solutions has been necessary to terminate outbreaks caused by $C$. difficile ${ }^{161}$ and Acinetobacter baumanii. ${ }^{153}$

Although the ICU environment cannot be made microbe free, certain organizational, architectural, and environmental issues must be addressed with the design or remodeling of an ICU. The capacity to systematically improve the care of critically ill patients and prevent nosocomial infection requires a structural foundation on which the processes of care can be optimized (i.e., make it easy for HCWs to do it right and difficult to do it wrong). Accountability for compliance with critical policies and procedures and ongoing assessment of outcomes needs to be built into the administrative structure of the ICU.

An ICU must be adequately staffed to allow the processes of care to be carried out but also assure a high level of compliance with essential infection control measures such as hand hygiene and barrier isolation. Adequate staffing cannot be overemphasized; numerous studies have found greatly increased rates of nosocomial infection when ICUs are staffed suboptimally or when staffing requirements are met with temporary personnel who are unfamiliar with ICU infection control policies and procedures. ${ }^{162,163}$ In a large nosocomial outbreak of Enterobacter cloacae infection in a neonatal ICU, Harbarth and colleagues ${ }^{164}$ found that infection rates during periods of understaffing were strikingly higher than during periods with adequate levels of staffing ( $R R=6,95 \% \mathrm{CI}=2.2$ to 16.4). The effects of understaffing are likely multiple; however, erosion of basic hygienic practices with excessive patient-to-staff ratios likely explains much of this phenomenon. ${ }^{165}$

Many of the published recommendations for ICU architectural design ${ }^{166}$ are empiric, and evidence that they reduce rates of nosocomial infection is, by and large, lacking. Although more research is necessary before specific features of ICU design achieve a level of evidence sufficient for an evidence-based guideline, certain facets of the ICU layout deserve attention:

- ICUs should be located in areas that limit traffic flow to essential ICU personnel.

- ICU facilities should be designed with ICU professionals in mind, ensuring appropriate space, resources, and environment for day-to-day operations. ${ }^{166}$ Recognizing the growing variety and complexity of life support equipment required for the care of many patients, each cubicle or room should provide a minimum of $11 \mathrm{~m}^{2}$ per bed. ${ }^{167}$ The area should be large enough to accom- modate the bed and all equipment yet allow immediate access to the patient at all times from both sides of the bed. Adequate space must also be provided for storage of nursing supplies. Facilities for disposal of biohazardous waste (e.g., bedpan flushers); for cleaning, reprocessing, and storage of ICU equipment; and for storage of housekeeping supplies should be separate from patient care areas. Single-patient rooms may increase the likelihood of handwashing being done and improve compliance with isolation practices, reducing the risk of cross-infection. For example, Mulin and colleagues ${ }^{168}$ found that converting from an open unit to single rooms in their ICU greatly reduced rates of patient colonization with A. baumanii, and Shirani and colleagues ${ }^{169}$ found that renovation of their burn unit to include separate bed enclosures reduced rates of nosocomial infection by $48 \%{ }^{169}$

- Materials used for fixtures, furniture, and other surfaces should be smooth and easy to clean; surfaces made of porous materials foster bacterial colonization. ${ }^{170}$

- An adequate number of sinks must be available for convenient handwashing by ICU personnel. Ideally, a sink should be located at the entrance of each cubicle or patient room to encourage handwashing by all entering personnel who will have contact with the patient or the immediate environment. ${ }^{171,172}$ Separate sinks should be used for cleaning and reprocessing contaminated equipment. Sinks and sink drains are normally contaminated by pseudomonads,${ }^{173}$ although their role in the epidemiology of nosocomial infection is as yet unclear. However, sinks should be designed to minimize aerosol formation and splashback.

- All ICUs should be equipped with one or more class A isolation rooms, ${ }^{200}$ which include an anteroom for gowning and handwashing and the necessary modifications (negative pressure, roofline exhaust) to permit it to be used for patients with tuberculosis or other airborne infections such as chickenpox, measles, disseminated HSV infection or a highly contagious emerging pathogen such as the severe acute respiratory syndrome (SARS) human coronavirus. If an ICU treats bone marrow transplant patients or other patients with prolonged severe granulocytopenia, positive-pressure isolation rooms using high-efficiency particle-arrest (HEPA) filters should be available. Isolation rooms for patients with infections transmitted by the respiratory route or to protect profoundly granulocytopenic patients must be kept closed to maintain control over the direction of airflow.

- A centralized, filtered air-handling system that provides at least six room-exchanges per hour is essential. ${ }^{167,174}$ Ideally, each patient's room should have the capacity of being set at positive or negative pressure with respect to the rest of the unit; if it cannot be, the room should be maintained permanently at positive pressure.

A variety of microorganisms including bacteria, mycobacteria, fungi, and parasites can be isolated from hospital 
water and have been implicated in endemic and epidemic nosocomial infections. ${ }^{175}$ Many of these outbreaks were caused by bacteria typically thought of as "water" organisms such as $P$. aeruginosa, ${ }^{173}$ Stenotrophomonas maltophilia, ${ }^{176}$ and A. baumanii ${ }^{153,177,178}$; however, the most important and epidemiologically linked hospital water pathogen is the Legionella group. ${ }^{179}$

Nosocomial legionellosis was first described in $1979,{ }^{180}$ and it is estimated that up to $50 \%$ of cases of legionellosis are acquired in the health care setting, ${ }^{181}$ with a mortality rate that approaches $30 \% .{ }^{182}$ Contamination of hospital potable water remains underappreciated despite studies showing that Legionella species can be recovered from $12 \%$ to $70 \%$ of hospital water systems, ${ }^{183}$ and a number of studies in which nosocomial cases were identified only when specific diagnostic and surveillance methods were employed. ${ }^{184,185}$ Characteristics of hospital water systems that are associated with Legionella contamination include piping systems with dead-ends that facilitate stagnation, large-volume water heaters that result in inefficient heating of hospital water, sediment build-up, water heater temperatures $<60^{\circ} \mathrm{C}$ and tap water temperatures $<50^{\circ} \mathrm{C}$, maintaining water $\mathrm{pH}>8$ and receiving municipal water untreated with monochloramine. ${ }^{186-188}$

Despite the ubiquity of water systems colonized with Legionella species and studies demonstrating a correlation between the level of colonization and risk of infection, the CDC does not recommend routine surveillance of hospital water systems, ${ }^{181}$ although this stance is controversial. ${ }^{183}$ Researchers from Pittsburgh, Pennsylvania, and the Allegheny County Health Department have recommended a more proactive stepwise approach that involves initial surveillance of hospital water for Legionella contamination, regardless of the presence or absence of institutional nosocomial legionellosis, followed by continued surveillance based on the level of water contamination found or the presence of institutional legionellosis. ${ }^{183}$

Legionella species are resistant to chlorine and heat, making it challenging to eradicate them from contaminated hospital water systems. ${ }^{188}$ Attempts to hyperchlorinate hospital water have been partially successful if chlorine levels are continuously maintained between 2 and 6 parts per million at all times but produce rapidly accelerated corrosion of water pipes and are expensive. ${ }^{189}$ Thermal eradication is feasible, using a "heat-and-flush" method to raise water tank temperatures to greater than $70^{\circ} \mathrm{C}$ and distal water sites to $>60^{\circ} \mathrm{C}$ for short periods of time. ${ }^{190}$ Although effective, super-heating is labor intensive and there is the constant fear that patients or health care personnel may sustain scald injuries if they wash or shower with tap water during a flushing period. The use of technologies such as instantaneous steam heat for incoming water ${ }^{190}$ and ultraviolet light ${ }^{191}$ are technically feasible with newer hospital water systems but may be incompatible with older hospital water systems.

Perhaps the most attractive, effective, safe, and costefficient method for Legionella eradication may be the use of continuous copper-silver ionization systems to sterilize hospital water systems. These systems have been well studied over the past decade and have proved to be highly effective for reliably eradicating Legionella contamination of hospital water and, most importantly, for eliminating nosocomial legionellosis in institutions when other interventions have failed. ${ }^{192}$ In our own institution, two clusters of nosocomial legionellosis prompted a retrospective review that identified 10 cases over a 11-year period. Surveillance of the hospital water system found that $75 \%$ of all samples contained low levels of L. pneumophila, which were shown to be clonally related to the 10 cases of nosocomial legionellosis. Installation of a continuous copper-silver ionization system led to complete eradication of Legionella from water samples, and no further cases of nosocomial legionellosis have been identified at our institution since 1995, among 255,000 patients hospitalized.

\section{Reliable Sterilization Procedures, Chemical Disinfectants, and Antiseptics}

Reliable sterilization, disinfection, and antisepsis embrace virtually all measures aimed at prevention of nosocomial infection. Critical objects, which are introduced directly into the bloodstream or into other normally sterile areas of the body, such as surgical instruments, cardiac catheters, and implanted devices, must be reliably sterile and sterilized with steam, gas, hydrogen peroxide gas, or chemical sterilization. Semicritical items, which come into contact with intact mucous membranes, such as fiberoptic endoscopes, endotracheal tubes, or ventilator circuit tubing, can be decontaminated between patients by pasteurization or the use of high-level chemical disinfection with glutaraldehyde, peracetic acid, hydrogen peroxide, ethyl alcohol, or hypochlorite. Noncritical items, which normally come into contact only with intact skin, such as blood pressure cuffs or electrocardiograph electrodes, require hygienic cleansing or low-level disinfection with an iodophor, hypochlorite, quaternary ammonium or phenolic disinfectants, or alcohol. ${ }^{193,194}$ The lone exception to this classification scheme is devices that pose a risk of transmitting prion-related diseases. Transmissible spongiform encephalopathies such as Creutzfeldt-Jakob disease (CJD) and variant CJD (vCJD) have gained considerable attention over the past decade and have only recently been addressed in published disinfection and sterilization guidelines. ${ }^{195}$ Prions are not readily inactivated by conventional disinfection and sterilization procedures. ${ }^{193}$ As a result, devices that pose a risk for transmission of prionrelated diseases should undergo special sterilization procedures after cleaning that involve sodium hydroxide followed by low-temperature autoclaving $\left(121^{\circ} \mathrm{C}\right)$ or hightemperature autoclaving $\left(132^{\circ} \mathrm{C}\right.$ for 1 hour or $134^{\circ} \mathrm{C}$ for 18 minutes). ${ }^{194}$ Despite concerns that procedures involving semicritical items such as endoscopes and bronchoscopes may pose a risk for transmission of prion-related infections, there has not been a single report of CJD or vCJD associated with these devices. As a result, current guidelines recommend that only critical items and semicritical items that have come in contact with neurologic tissue (e.g., brain, spinal cord, eye tissue) should undergo special prion inactivation sterilization procedures. ${ }^{194,196}$ 
Numerous epidemics of gram-negative infection have been described in association with respiratory therapy equipment, ${ }^{94,95}$ diagnostic equipment such as bronchoscopes and endoscopes, ${ }^{95,99-101}$ and solutions used for cutaneous antisepsis. ${ }^{197,198}$ Most of these outbreaks were traced to improper procedures or malfunction of automated systems used for the disinfection and sterilization of medical devices, although a number of epidemics in years past arose as a result of extrinsic contamination of solutions used for cutaneous antisepsis. ${ }^{197,198}$ For these reasons, the importance of strict adherence to recommended policies and procedures for cleaning and reprocessing medical equipment used in the ICU cannot be overemphasized.

Endoscopes and bronchoscopes are essential diagnostic and therapeutic instruments in the ICU. Although most postendoscopy nosocomial infections are caused by inoculation of colonizing mucosal flora into normally sterile, vulnerable anatomic sites during the procedure, numerous epidemics have been traced to contaminated endoscopes. ${ }^{95,99-101}$ Following use for bronchoscopy, endoscopes are typically contaminated with $6 \times 10^{4}$ colony-forming units $(\mathrm{CFUs} / \mathrm{mL}) .{ }^{199}$ All endoscopes are considered semicritical medical devices by the Spaulding classification and therefore require high-level disinfection following use. ${ }^{196}$ In order to ensure their safe use, flexible endoscopes should be reprocessed with the following procedures: (1) physical cleaning to reduce microbial bioburden and remove organic debris; (2) high-level disinfectionglutaraldehyde and automated chemical sterilizing systems that use peracetic acid are most commonly used in the United States-with adequate contact time between the disinfectant and device surface; (3) following disinfection, rinsing with sterile or filtered tap water to remove dis-infectant residue; (4) flushing of all channels with $70 \%$ to $90 \%$ ethyl or isopropyl alcohol; and (5) drying with forced air. ${ }^{196}$ Devices used with endoscopes that violate mucosal barriers, such as biopsy forceps, need to be reprocessed as critical medical items with full sterilization. ${ }^{196}$ Other devices used in the delivery of respiratory care are also considered semicritical under the Spaulding classification and therefore should be reprocessed in a manner similar to endoscopes prior to reuse. ${ }^{131}$

Iodophors (e.g., $10 \%$ povidone-iodine), until recently, have been the most common agents used for cutaneous disinfection in North America. However, a large, prospec- tive, randomized trial of cutaneous antiseptics used for drawing blood cultures recently showed that chlorhexidine was superior to $10 \%$ povidone-iodine and was associated with a more than twofold reduced rate of contaminated blood cultures $(\mathrm{OR}=0.40,95 \%$ CI 0.21 to $0.75, P=.004){ }^{200}$ Moreover, a recent meta-analysis examining the impact of different cutaneous antiseptic agents found that chlorhexidine was superior to povidone-iodine for both the prevention of intravascular catheter colonization and catheter-related bloodstream infection. ${ }^{201}$ On the basis of these and other recent studies, ${ }^{202,203}$ chlorhexidine-containing solutions are the preferred cutaneous antiseptics for insertion of intravascular devices in the ICU. ${ }^{129}$ Whatever agent is used, it is essential that it be applied with vigorous scrubbing for a minimum of 1 minute to allow adequate time for germicidal activity.

\section{Hand Hygiene}

The major reservoir of nosocomial infection in the ICU is infected or colonized patients, and the major mode of spread of most nosocomial bacterial pathogens, many viruses, and even Candida from patient to patient is by transient carriage on the hands of medical personnel (see Fig. 51-3). Studies in our center of hand carriage of nosocomial pathogens by ICU personnel, using a simple rinse technique to quantify the transient flora, ${ }^{204}$ have shown that, on average, approximately 60,000 CFUs (or 4.6 logs) are recovered from the hands of ICU personnel randomly sampled (Table 51-6). Nearly half of persons cultured at any point in time will be found to be carrying gram-negative bacilli, and $10 \%$ will be carrying S. aureus. ${ }^{205}$ Serial culturing has shown that all ICU personnel, at various times, carry gram-negative bacilli and that nearly two thirds carry $S$. aureus. Carriage of both gram-negative bacilli and $S$. aureus is typically transient: sampling persons every other day over a prolonged period has shown $S$. aureus or the same gram-negative species in consecutive cultures only $16 \%$ of the time; prolonged carriage of a single gram-negative species seems to be rare-but has been reported. ${ }^{206}$

Hygienic handwashing before undertaking invasive procedures, handling open wounds, or having manual contact with high-risk patients (e.g., newborns or patients in ICUs) or after touching a source or object likely to be contaminated has been recognized since the time of Semmelweis and Lister as one of the most basic and

\begin{tabular}{|c|c|c|c|}
\hline & All Microorganisms & Gram-Negative Bacilli & Staphylococcus aureus \\
\hline $\begin{array}{l}\text { Mean } \log _{10} C F U \pm S D \text {, recovered from persons' hands* } \\
\text { (range of individuals' means) }\end{array}$ & $\begin{array}{l}4.59 \pm 0.69 \\
(3.31-5.76)\end{array}$ & $\begin{array}{l}1.04 \pm 0.44 \\
(0.29-1.93)\end{array}$ & $\begin{array}{l}0.44 \pm 0.44 \\
(0-1.45)\end{array}$ \\
\hline$\%$ All cultures positive & 100 & 44.5 & 11.2 \\
\hline$\%$ All individuals positive at least once* & 100 & 100 & 64 \\
\hline
\end{tabular}


important infection control measures. Despite universal acknowledgement of handwashing as a cornerstone of nosocomial infection control programs, compliance rates much above $50 \%$ have been difficult to achieve and handwashing rates among HCWs have ranged from 9\% to $50 \%$ in numerous observational studies. ${ }^{165,207,208}$ Recent investigations have undertaken to better understand the reasons for poor compliance in the face of the compelling evidence that hand hygiene is essential for prevention of nosocomial infection, ${ }^{165}$ identifying cutaneous irritation, inconvenient sink location, time constraints, high workload, and understaffing. Of concern, risk factors for noncompliance with hand hygiene include being a physician (rather than a nurse); working in an ICU; and, paradoxically, engaging in patient-care activities with a high risk of cross-transmission. ${ }^{165}$ Interventions to redress these deficiencies have included targeted education; feedback; convenient location of sinks and hand hygiene agents; use of alternative, less irritative hand hygiene agents; hand care lotions or creams ${ }^{209}$; and patient education. ${ }^{210}$

Studies done with working hospital staff have shown that hygienic handwashing with an antiseptic-containing agent reduces the count of microorganisms on the hands of the user far more effectively than handwashing with a nonmedicated soap. ${ }^{204}$ Repeated use of some antiseptics such as chlorhexidine has a cumulative suppressive effect on the transient hand flora. Routine use of an antisepticcontaining handwashing agent could, in theory, enhance the effectiveness of the handwashing that is done. Moreover, if an agent that exhibits prolonged antimicrobial activity, such as chlorhexidine, is used, it might also confer protection against contaminants acquired between handwashings. ${ }^{204}$ However, antiseptic-containing handwashing agents are more expensive and often more irritating to the skin. Irritation can result in dermatitis and, paradoxically, increased colonization by gram-negative bacilli. ${ }^{211}$

Clearly, antiseptic-containing soaps are more effective in removing microorganisms from the hands of users, but will routine use of these agents for hygienic handwashing reduce the incidence of nosocomial infection in patients? Discontinuation of hexachlorophene for handwashing by personnel and bathing of infants in the United States in 1973 was followed by a marked upsurge in $S$. aureus infections in nurseries, ${ }^{212}$ and use of chlorhexidinecontaining handwashing agents was considered an essential measure for control of hospital outbreaks caused by multiply resistant Klebsiella ${ }^{213}$ and MRSA. ${ }^{214,215}$ However, since Semmelweis' study, few studies have prospectively evaluated the efficacy of antiseptic-containing handwashing agents for reducing endemic nosocomial infections, particularly infections caused by gram-negative bacilli. $^{211,216}$

In 1982 a comparative sequential trial of three handwashing agents-a nonmedicated tissue soap, 10\% povidone-iodine (Betadine Scrub), and $4 \%$ chlorhexidine (Hibiclens) - was undertaken in the trauma-surgical ICU of the University of Wisconsin Hospital. ${ }^{211}$ Each agent was used exclusively for approximately 6 weeks, during which time hand cultures of ICU personnel were done at random and surveillance of infection in patients was carried out.
Risk factors for infection in patients hospitalized during the use of each agent were comparable: Nearly two thirds of the patients in each period required ventilatory support and hemodynamic monitoring, and almost all had urinary catheters. The incidence of nosocomial infection in all groups was expectedly high, but it was 30\% lower during the use of the two antiseptic-containing handwashing agents than during the use of the nonmedicated soap $(P<.001)$. Povidone-iodine was irritating to the hands of most staff, and chlorhexidine had a slightly drying effect but was well tolerated, comparable with the nonmedicated soap.

In a similar study at the University of Iowa Hospital, Massanari and Heirholzer ${ }^{216}$ did not find significant differences in the rates of nosocomial infection when nonmedicated soap was used exclusively as compared with alternating cycles during which $4 \%$ chlorhexidine (Hibiclens) was used in surgical ICUs; however, the incidence of infection in the medical ICU was $50 \%$ lower during use of chlorhexidine $(P<.05)$.

In the largest multiple-crossover prospective study1894 adult patients in three ICUs_-of the relative efficacy of antiseptic-containing handwashing agents used by personnel in ICUs, Doebbeling and colleagues ${ }^{217}$ found that the use of $4 \%$ chlorhexidine (Hibiclens) was associated with a $30 \%$ reduction in nosocomial infections $(\mathrm{OR}=0.73)$, as contrasted with rates when a $60 \%$ alcohol hand-rinsing agent (Cal-Stat) was used. Both regimens were well tolerated.

Recently, alcohol-based, waterless hand rubs have become the agents of choice for hand hygiene and are now universally used in U.S. hospitals because of their convenience and broad-spectrum activity. ${ }^{210}$ Alcohols have the most rapid and pronounced bactericidal action and greatly reduce the time needed for hand disinfection. A vigorous 1-minute rubbing with a sufficient volume of alcohol to wet the hands completely has been shown to be highly effective at reducing the density of skin flora. ${ }^{218}$ Ethanol, iso- and n-propanol are the constituents of most commercially available alcohol-based hand rubs; at equal concentrations, n-propanol is most effective and ethanol, the least. However, all have limited efficacy with gross soilage so that visibly soiled hands should always be washed with antiseptic soap and water. ${ }^{171}$ Moreover, at least $3 \mathrm{~mL}$ of an alcohol-based rub is necessary to completely coat the hands and achieve optimal degerming. The use of alcohol hand rubs or gels will be augmented by making conveniently located calibrated dispensers widely available. However, many HCWs prefer individual containers that can be carried in a pocket, which makes it difficult to ensure that an adequate volume is used with each application.

Few trials have been conducted to evaluate the efficacy of alcohol-containing hand rubs for reducing nosocomial infection. Most are quasi-experimental before-after studies, and most have shown a short-term reduction in nosocomial infection rates with use of alcohol-containing hand rubs. ${ }^{172,219,220}$

The major factor limiting acceptance of alcohol products for hand antisepsis in the past was desiccation and 
irritation of skin. This is now obviated by incorporating emollients into alcohol-based hand rubs, which has enhanced acceptance by HCWs and may augment antibacterial activity by slowing the evaporation of alcohol. ${ }^{221}$ A recent randomized clinical trial in 50 ICU HCWs compared a conventional $2 \%$ chlorhexidine gluconate wash with water to a waterless alcohol-based hand rub $(61 \%$ ethanol with emollients) and showed that use of the waterless alcohol-based product produced significantly less skin scaling and irritation ${ }^{222}$; unfortunately, degerming was not assessed.

A recent review describes in detail the various hand hygiene agents available and their spectrum of activity. ${ }^{210}$ Recommendations for hand hygiene by the CDC have recently been published (Table 51-7), ${ }^{171}$ emphasizing hand antisepsis with an antiseptic-containing soap or detergent or an alcohol-based hand rub: (1) before and after direct contact with patients or the environment and equipment in the immediate vicinity of the patient and (2) before performing invasive procedures such as insertion of an intravascular device or urinary catheter. Use of skin care products-lotions or creams - to minimize irritant contact dermatitis associated with frequent handwashing and improve compliance with hand hygiene practices is highly recommended.

Institutional commitment is essential to improve compliance with recommended hand hygiene practices. The CDC guideline recommends that institutions (1) monitor and record adherence to hand hygiene by ward or service; (2) provide feedback to HCWs about their performance and (3) monitor the volume of alcohol hand rubs used per 1000 patient-days. ${ }^{171}$

Clearly, further studies are necessary, particularly large comparative trials in which rates of nosocomial infection, rather than levels of cutaneous colonization, are used as the index of comparison. In the meantime the available data indicate that routine use of a chlorhexidinecontaining product or alcohol-containing product will be more effective than use of a nonmedicated soap for hand hygiene in the high-risk areas of the hospital, such as ICUs, where cross-infection is most likely to occur.

\section{Isolation Precautions for \\ Communicable Infections}

Isolation, the use of special precautions in the care of infected patients, is the only means of curtailing the spread of contagious microorganisms and preventing epidemics, especially in ICUs, where the risk of crossinfection is highest. Although requiring all persons entering an infected patient's room to wear gloves and a gown, possibly even a mask, may seem ritualistic and almost archaic, each aspect of the isolation procedure is directed at interrupting a potential mode of spread and is based on the known epidemiology of the infecting organism. ${ }^{223}$ To be maximally effective, however, isolation procedures require compliance by each person coming into contact with the patient, including physicians. Isolation is also indicated, usually for the entirety of hospitalization, for all patients infected or known to be colonized by antibioticresistant nosocomial pathogens such as MRSA, gram-nega-
Table 51-7. Recommendations for Routine Hand Hygiene from the Centers for Disease Control and Prevention Guideline

\section{Recommendation}

Level of Evidence*

- When hands are visibly dirty or contaminated with proteinaceous material or are visibly soiled with blood or other body fluids, wash hands with either a nonantimicrobial soap and water or an antimicrobial soap and water

If hands are not visibly soiled, use an alcohol-based hand rub or, alternatively, wash hands with an antimicrobial soap and water for the following situations:

Before direct contact with patients

Before putting on sterile gloves when inserting a central vascular catheter

Before inserting urinary catheter, peripheral vascular catheter, or other invasive procedure not requiring surgery

After contact with patient's intact skin

After contact with body fluids, mucous membranes, and wound dressings if hands are not visibly soiled

Moving from a contaminated body site to a clean body site during patient care

After contact with inanimate objects in the immediate vicinity of the patient

After removing gloves

Before eating and after using a restroom, wash hands with a nonantimicrobial soap and water or with an antimicrobial soap and water

- Antimicrobial-impregnated wipes are not a substitute for using an alcohol-based hand rub or antimicrobial soap

- If exposure to Bacillus anthracis, wash II hands with nonantimicrobial soap and water or antimicrobial soap and water

${ }^{*}$ Categorization of recommendations: IA: strongly supported for implementation and strongly supported by well-designed experimental, clinical or epidemiologic studies. IB: strongly recommended for implementation and supported by certain clinical or epidemiologic studies and by strong theoretical rationale. IC: required for implementation, as mandated by federal or state regulation or standard. II: suggested for implementation and supported by suggestive clinical or epidemiologic studies or by strong theoretical rationale. No recommendation: unresolved issue: practices for which insufficient evidence or no consensus exists about efficacy. Modified from Boyce JM, Pittet D: Recommendations of the Healthcare Infection Control Practices Advisory Committee and the HICPAC/SHEA/ APIC/IDSA Hand Hygiene Task Force. MMWR Recommend Rep 2002;16:1-45. 
tive bacilli resistant to aminoglycosides or third-generation cephalosporins, or VRE; in such cases, isolation has been shown to be effective in reducing endemic infections ${ }^{224,225}$ (Figs. 51-5 and 51-6) and in controlling outbreaks. ${ }^{225}$

\section{Isolation Systems}

Most U.S. hospitals subscribe to one of two CDC isolation systems developed by panels of experts. The simplest system, category-specific isolation precautions, issued by the CDC in $1970,{ }^{223}$ groups diseases in seven categories by infections for which similar precautions are indicated: wound and skin precautions, enteric precautions, discharge precautions, blood precautions, respiration isolation, strict isolation, and protective isolation. Guidelines for disease-specific isolation precautions, issued in $1983,{ }^{226}$ consider each infectious disease individually, so only those precautions indicated to interrupt transmission of that specific disease are used. Disease-specific precautions minimize unnecessary isolation procedures; however, they are more complicated and may be implemented most effectively by a computerized system.

An alternative, simpler system, body substance isolation, has gained adherents and focuses on the isolation of potentially infectious body substances, such as blood, feces, urine, sputum, wound drainage, and other body fluids, of all patients through the use of simple barrier precautions-primarily gloves, gowns, plastic aprons, and masks or goggles. These barriers should be used when potentially infectious secretions are likely to soil or splash the clothing, skin, or face of the HCW. ${ }^{227}$ Body substance isolation provides sufficient flexibility to augment the basic precautions taken with each patient, as needed, and adds private rooms with masks for infections transmitted by the airborne route. A criticism of this simpler system

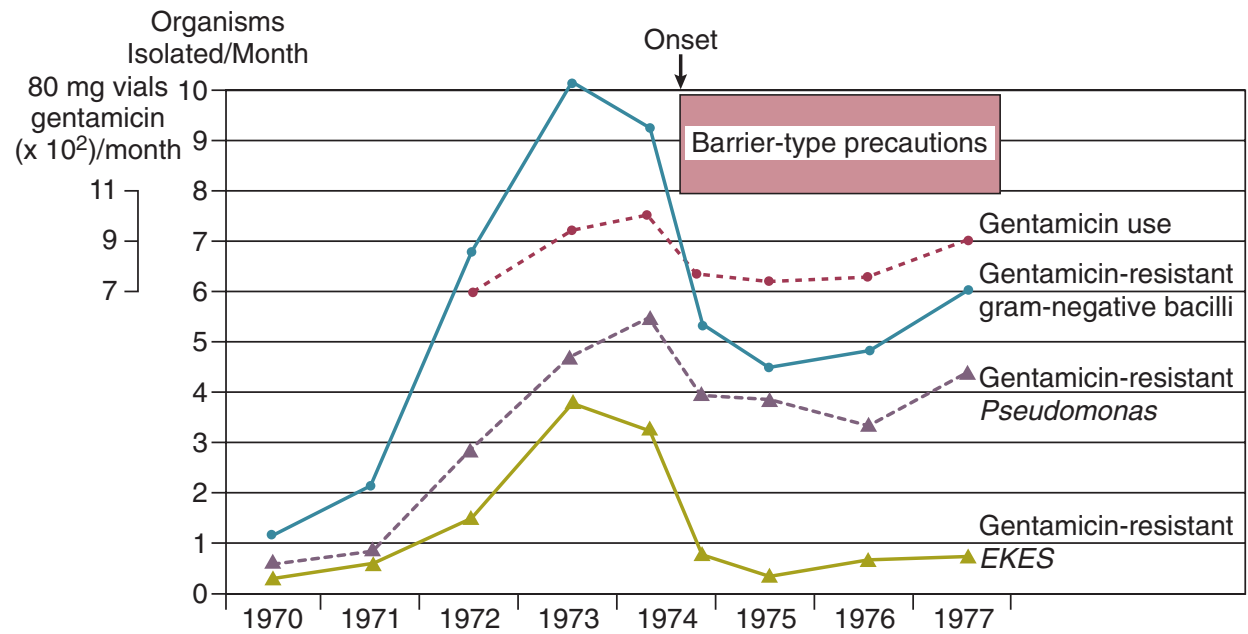

Figure 51-5. Impact of implementing barrier-type precautions (gown and gloves) with patients known to be colonized or infected by gram-negative bacilli resistant to gentamicin. Frequency of infections by gentamicin-resistant gram-negative bacilli and gentamicin use at Michael Reese Medical Center, 1970-1977. Data are plotted as the monthly average, and the averages for the first 7 and last 5 months of 1974 are plotted separately to demonstrate the effect of barrier-type precautions implemented in August 1974. EKES, Escherichia coli, Klebsiella pneumoniae, Enterobacter species, and Serratia species. (From Weinstein RA, Nathan C, Gruensfelder R, et al: Endemic aminoglycoside resistance in gram-negative bacilli: epidemiology and mechanisms. J Infect Dis 1980;141:338-345.)

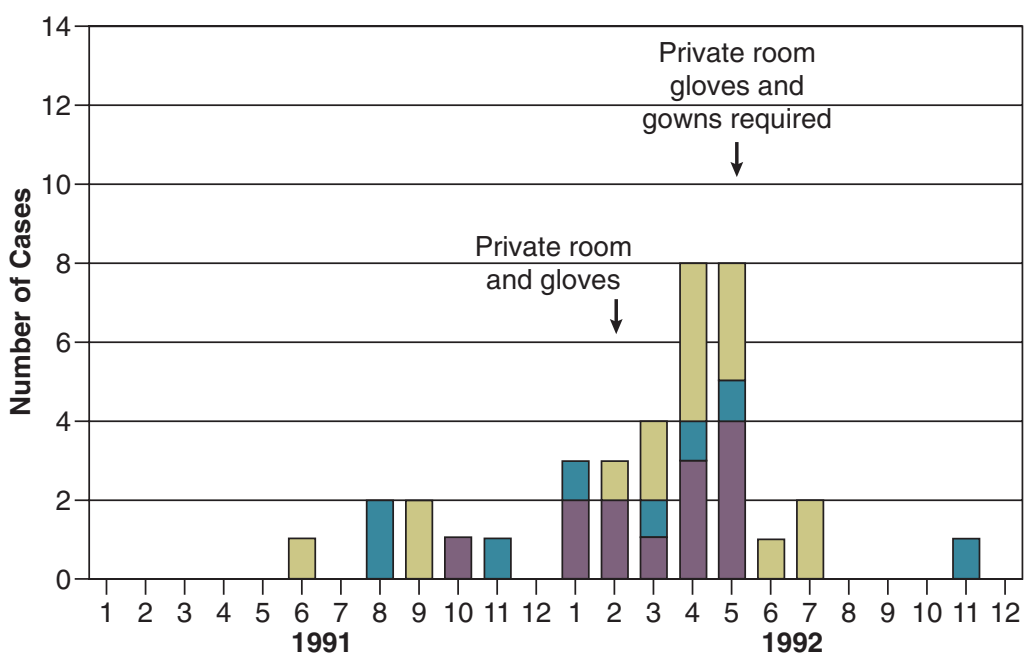

Figure 51-6. Impact of specific control measures on an institutional outbreak of vancomycin-resistant Escherichia faecium. Cases, by date of first positive culture for the epidemic strains (from January 1991 to December 1992). Number of cases $=$ case patients in the intensive care unit (ICU) at time of first positive culture for the epidemic strain $\times$ other patients with previous exposure to the ICU $\times$ case patients never in the ICU. (From Boyce JM, Opal SM, Chow JW, et al: Outbreak of multidrug-resistant Enterococcus faecium with transferable vanB class vancomycin resistance. J Clin Microbiol 1994;32:1148-1153.) 
has been the reduced emphasis on handwashing when gloves are removed. ${ }^{228}$

The most recent CDC guideline, currently in draft form, ${ }^{174}$ separates basic precautions into (1) standard precautions designed for the care of all patients in hospitals, regardless of their diagnosis or presumed infection status, and (2) additional transmission-based precautions designed for the care of specified patients who are known or suspected to be infected with highly transmissible or epidemiologically important pathogens. Standard precautions synthesize the major features of universal blood and body fluid precautions and are designed to reduce the risk of transmission of microorganisms from patient to patient and from patient to HCW, from both recognized and unrecognized sources of infection in the hospital. Transmission-based precautions are divided into three subgroups on the basis of the mode of transmission: contact precautions, droplet precautions, and airborne precautions. Contact precautions are recommended with multidrug-resistant bacteria that can be acquired by contact with the colonized patient or environmental surfaces or objects. Droplet precautions provide additional measures for transmission by large-particle droplets, such as during suctioning or bronchoscopy. Airborne precautions are added to standard precautions for care of patients with tuberculosis and other microorganisms transmitted by the airborne route. In general, transmission-based precautions usually specify a private room-always for airborne precautions.

\section{Special Issues in the ICU}

An environmental issue pertaining to isolation may be most relevant in the ICU, namely, the greater potential for fomites or environmental surfaces to contribute to the spread of nosocomial infection, especially with antibioticresistant microorganisms. Although previous studies have not been able to demonstrate that the inanimate hospital environment, particularly surfaces, walls, or floors, contribute materially to the occurrence of nosocomial infection, ${ }^{154,155}$ accumulating evidence suggests that this may not necessarily be true for ICUs, where uniform exposure to invasive devices makes patients unduly susceptible. A number of careful studies of the epidemiology of ICUacquired infection with resistant organisms such as MRSA,${ }^{155,156}$ C. difficile, ${ }^{80,157}$ and VRE ${ }^{152,158,229}$ have shown heavy contamination of the inanimate environment immediately contiguous to the patient by strains implicated in nosocomial infections occurring in patients. Even if gloves are being worn as part of protective isolation or universal precautions, the possibility of transmission of microorganisms from the environment to patients on the gloved hands of HCWs is real. Prolonged wearing of gloves in the ICU, which is common, may increase the risk of nosocomial cross-infection, expanding the epidemiologic role of the inanimate environment with certain pathogens such as MRSA or VRE. ${ }^{229}$

Similarly, the use of common stethoscopes, sphygmomanometers, or electronic thermometers with multiple patients provides further opportunity for organisms to spread. Although stethoscopes are commonly contami- nated by nosocomial organisms, ${ }^{230}$ their role in crossinfection is less clear. ${ }^{230}$ On the other hand, spread of VRE $^{231}$ and $C$. difficile ${ }^{232}$ has been traced to contamination of electronic thermometers. All surfaces contiguous to the ICU patient should be wiped down with the general hospital disinfectant at least daily, and each ICU patient should have a dedicated stethoscope and sphygmomanometer. The use of electronic temperature measuring devices on multiple patients within an ICU bears reevaluation, unless stringent efforts are made to assure reliable decontamination of the device after each use.

As discussed, many nosocomial infections appear to derive from organisms carried on the hands of ICU personnel, who during the working day have contact with multiple patients. To improve nursing care and reduce the risk of cross-infection, ICUs must have an adequate number of staff. Although the optimal nurse/patient ratio for patients in an ICU is not known, increased rates of infection and outbreaks have occurred when nurses have been assigned to multiple critically ill patients who require complicated nursing care. ${ }^{163}$ One-to-one nurse/ patient ratios may significantly reduce the risk of crossinfection.

To contain the spread of certain resistant organisms in the ICU (e.g., MRSA, VRE), cohort nursing is strongly recommended. In cohort nursing, the care of patients known to be infected (or colonized) by the organism is provided by nurses (and respiratory therapists) who will not provide care during that shift for noninfected patients, and the nursing care of noninfected patients is restricted to personnel who will not have contact with infected patients, except in an emergency. Cohorting of patients known to be colonized or infected with MRSA is widely practiced but has not been adequately studied. In one recent prospective study, the authors found that there was no evidence of increased transmission of MRSA when patients were not cohorted. ${ }^{233}$

\section{Tuberculosis}

The upsurge in tuberculosis since 1985, particularly the numerous nosocomial outbreaks caused by multidrugresistant strains, ${ }^{70-72,234,235}$ demonstrates the importance of isolation precautions to prevent the spread of tuberculosis within hospitals, especially within ICUs. ${ }^{126} \mathrm{New}$ guidelines $^{126}$ reemphasize the importance of air control by mandating the use of private negative-pressure rooms, combined with the use of ultraviolet lights or ventilatory modifications in which all air exiting the room is either filtered or exhausted directly to the roofline, away from hospital intake vents. Isolation room doors must be kept closed to maintain control over the direction of airflow, and all persons who enter a room in which tuberculosis isolation precautions are in effect must wear a disposable particulate respirator such as a dust-mist mask or a HEPAfilter mask. Gowns and gloves usually are not indicated. All ICUs should have one or more negative-pressure isolation rooms for the care of patients requiring respiratory isolation for tuberculosis and other airborne infections such as chickenpox or disseminated herpes zoster, disseminated HSV infection or emerging, highly contagious 
airborne infections such as SARS. To reduce the risk of contaminating a ventilator or discharging $M$. tuberculosis into the environment, when mechanically ventilating a patient with suspected or confirmed pulmonary tuberculosis, a bacterial filter capable of filtering particles $\geq 0.3 \mu \mathrm{m}$ in size, with a filter efficacy of greater than $95 \%$, should be placed on the patient's endotracheal tube or at the expiratory side of the breathing circuit of a ventilator. ${ }^{126}$ ICU patients with tuberculosis not requiring mechanical ventilation should wear a surgical mask if leaving the negative-pressure isolation rooms for radiographic or other procedures. ${ }^{126}$

\section{Standard Precautions}

The world epidemic of AIDS and evidence that more than 1 million persons in the United States are silent carriers of the human immunodeficiency virus (HIV) have engendered great concern among HCWs regarding the risk of exposure to HIV in the workplace. In 1987 the CDC and the Department of Labor issued detailed guidelines for Universal Blood and Body Fluid Precautions ${ }^{236,237}$ to prevent exposure of HCW workers and patients to potentially hazardous blood or body fluids. Universal precautions were based on the concept that all blood and body fluids that might be contaminated with blood should be treated as infectious because patients with bloodborne infections can be asymptomatic or unaware they are infected. The relevance of universal precautions to other aspects of disease transmission was recognized, and in 1996 the CDC expanded the concept and changed the term to Standard Precautions. ${ }^{238}$ Standard precautions integrate and expand the elements of universal precautions into a standard of care designed to protect health care personnel and patients from pathogens that can be spread by blood or any other body fluid, excretion, or secretion. Standard precautions apply to contact with (1) blood; (2) all body fluids, secretions, and excretions (except sweat), regardless of whether they contain blood; (3) nonintact skin; and (4) mucous membranes.

Gloves are recommended for venipunctures, insertion of intravascular devices, and whenever it can be anticipated that the hands could become contaminated by blood or another high-risk body fluid. If there is potential for splatter or contamination of clothing, a gown is added. When there is potential for aerosolization of body fluids, such as during surgery, intubation, endoscopy, or insertion of an arterial catheter, a mask and eye shielding are included. Because the vast majority of occupationally related HIV infections have involved needle sticks or other sharps injuries, every effort must be made to avert such injuries that could result in percutaneous inoculation of HIV or other bloodborne viruses. ${ }^{23,239}$

Because prophylactic use of barrier precautions appears to be of some benefit for prevention of nosocomial infection ${ }^{169,240,241}$ and all U.S. hospitals are currently mandated to follow standard precautions, it has been suggested that the use of gloves for all patient contacts, as is now common in many U.S. hospitals, should implicitly reduce the risk of nosocomial infection in general. However, this has not been demonstrated and there is concern that standard precautions might paradoxically increase the risk of nosocomial cross-infection. ${ }^{242}$ In most U.S. hospitals it is still common to observe ICU personnel, many of whom routinely wear gloves for all patient contacts to protect themselves, put on gloves, touch heavily contaminated areas (e.g., an open wound or tracheostomy), and then, without removing the gloves, proceed to write in the patient's chart, answer the telephone, or care for another patient. This occurs because the health care providers have forgotten that although the gloves may protect themselves, the gloves must be immediately discarded after use to prevent cross-contamination of hazardous pathogens to other vulnerable sites on the same patient or transmission to other patients or the ICU environment. Before the era of AIDS and universal precautions, health care professionals were oriented toward protecting the patient and likely to wash their hands when exposed to potential contamination. Now the focus is centripetal, and many HCWs unfortunately view all precautions as measures to protect themselves. Thus prolonged wearing of gloves can result in heavy contamination of the gloves ${ }^{243}$ and increase the risk of nosocomial cross-infection among patients. ${ }^{229,244}$ It also puts the HCW worker at increased risk of dermatitis and allergic reactions to glove material. ${ }^{245}$ Standard precautions do not obviate the need for designated isolation precautions for patients with communicable infections. The greatly expanded use of gloves as part of standard precautions in hospitals must now be accompanied by educational programs on how to use gloves effectively and in a manner that will not jeopardize patients. Staff must be strongly encouraged to wash their hands after removing gloves, especially after performing a bloody procedure, because blood often penetrates defects in gloves and can be found on the hands of the wearer. ${ }^{246}$ Moreover, if the gloved HCW has had hands-on contact with a patient colonized by MRSA or VRE, the process of removing the gloves will result in contamination of the hands of the HCW by these organisms up to one third of the time.

\section{Antibiotic Stewardship}

There is a world crisis in antibiotic resistance (Fig. 51-2), ${ }^{247,248}$ which reflects in greatest measure the heavy use of systemic antibiotics worldwide over the past 30 years, especially in hospitals. Antimicrobial therapy has its greatest ecologic impact in the close confines of the ICU. Most nosocomial outbreaks caused by antibioticresistant microorganisms ${ }^{249,250}$ have occurred in patients hospitalized in an ICU. Antibiotic pressure, which promotes the exchange of genes encoding drug resistance by a variety of transfer mechanisms (Fig. 51-7), ${ }^{251}$ has been shown to be the single most important factor predisposing patients to nosocomial infection with resistant organisms. Modern-day ICUs are the breeding grounds for the multiply resistant bacteria that are now being encountered in hospitals throughout the world: methicillin-resistant staphylococci; VRE; Enterobacter, Serratia, Citrobacter, Proteus-Providencia, and $P$. aeruginosa resistant to fluoroquinolones, aminoglycosides, or extended-spectrum beta-lactams. ${ }^{55-58,247}$ Broad-spectrum antimicrobial therapy 


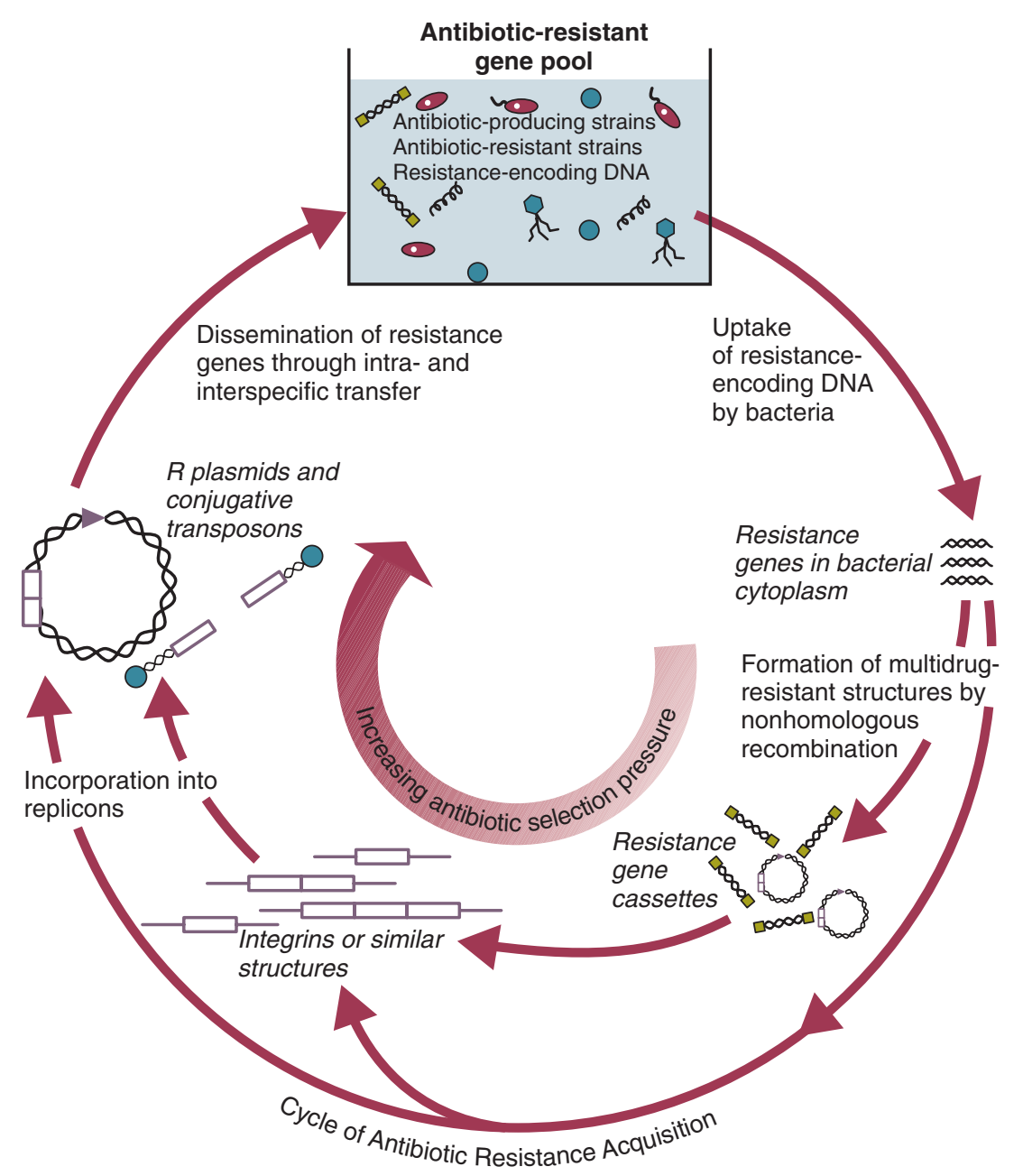

Figure 51-7. Schematic depicting the route by which antibioticresistant genes are acquired by bacteria in response to selection pressure of antibiotic use. The resistance gene pool represents all potential sources of DNA encoding antibiotic-resistant determinants in the environment; this includes hospitals, farms, or other microenvironments where antibiotics are used to control bacterial development. After uptake of single- or double-stranded DNA by the bacterial host, the incorporation of the resistance genes into stable replicons (DNA elements capable of autonomous replication) may occur by several different pathways that have not yet been identified. The involvement of integrins, as shown here, has been demonstrated for a large class of transposable elements in the Enterobacteriaceae. The resulting resistance plasmids could exist in linear or circular form in bacterial hosts. The final step in the cycle, dissemination, is brought about by one or more gene transfer mechanisms. (From Davies J: Inactivation of antibiotics and the dissemination of resistance genes. Science 1994;264:375-382.) is the root cause of antibiotic-associated diarrhea and colitis caused by $C$. difficile. ${ }^{80}$

Clearly, antimicrobials are widely overused and misused; more than $75 \%$ of patients in U.S. ICUs, other than coronary care units, receive antimicrobial agents, whereas studies indicate that more than half of hospitalized patients receiving antimicrobial therapy have no evidence of infection or clear justification to be receiving antibiotics. ${ }^{252}$ Moreover, within ICUs, a high proportion of the antibiotics used are broad-spectrum-extended-spectrum penicillins, third-generation cephalosporins, carbapenems, aminoglycosides, or fluoroquinolones. Greater efforts must be directed to improving the use of systemic antibiotics, especially within ICUs.

JCAHO now mandates that hospitals periodically review their use of antimicrobial agents through the use of antimicrobial audits. ${ }^{31}$ Such audits should scrutinize the need for antimicrobial therapy-clear evidence of infection or clear justification for prophylactic use, the appropriateness of the regimen selected, and monitoring for therapeutic efficacy and side effects during therapy. ${ }^{253}$ Educational programs and institutional guidelines for antimicrobial use that permit the hospital staff to construct guidelines and policies based on local needs and judgments, aided by published criteria, have been shown to materially improve antimicrobial use within the hospi- tal. ${ }^{252,254}$ Other important methods for controlling antimicrobial use include a restricted formulary, the policies of the clinical microbiology laboratory on reporting of susceptibility testing, and automatic stop orders for surgical prophylaxis. ${ }^{252}$ Many institutions also place expensive or the most broad-spectrum drugs (e.g., third-generation cephalosporins, carbapenems, amikacin, ciprofloxacin, fluconazole, ganciclovir, lipid-associated amphotericin B) on a restricted list, requiring physicians who wish to use the agents to justify their use to a representative of the institutional antibiotic review committee. ${ }^{255}$ Such programs greatly reduce use of restricted antibiotics and are gaining ever-wider acceptance.

Excellent resources, ${ }^{256,257}$ including other chapters in this book (Chapters 21, 52), are available to guide the selection and use of anti-infective drugs in critically ill patients. However, several principles can reduce unnecessary antimicrobial therapy and improve the use of the drugs that are given:

1. Fever without other indications of infection should not mandate automatically beginning antimicrobial therapy in an ICU patient.

2. Unless antimicrobial therapy is being given for surgical prophylaxis, it is most likely being given for treatment of suspected or proved infection. Gram-stained smears, 
cultures, and other appropriate diagnostic tests, as indicated, should be done without fail before beginning antimicrobial therapy for treatment of presumed infection in an ICU patient.

3. Whenever antimicrobial therapy is begun, the reason should be documented in the patient's record (e.g., "for treatment of pneumonia," "for surgical prophylaxis").

4. When possible, a single drug and the most narrowspectrum drug or drugs should be used, especially if the infecting organism or organisms are known at the outset.

5. The need for continued antimicrobial therapy should be reassessed daily. If cultures identify the infecting microorganism or microorganisms, therapy should be modified, aiming for the most narrow-spectrum drug or drugs likely to be effective. If diagnostic studies are negative after 48 to 72 hours and the patient is not exhibiting signs of sepsis, antibiotic therapy should be discontinued, unless the patient is profoundly granulocytopenic.

6. Beyond monitoring for efficacy and adverse drug effects such as hypersensitivity or organ toxicity, it is essential that monitoring include surveillance for superinfection by resistant bacteria or Candida and for C. difficile diarrhea.

7. Surgical antimicrobial prophylaxis should not extend beyond 24 hours postoperatively ${ }^{258,259}$ and in most operations can be limited to a single dose. ${ }^{258}$

\section{SPECIFIC CONTROL MEASURES}

As noted earlier, most nosocomial infections, especially in immunologically competent patients and in ICUs, are causally related to surgical operations or exposure to invasive devices of various types (see Tables 51-2 and 51-3). Comprehensive guidelines for the prevention of infection with procedures or devices that pose the greatest risk (urinary catheters, ${ }^{130,260}$ endotracheal intubation and mechanical ventilatory support, ${ }^{131}$ intravascular catheters and infusion therapy, ${ }^{129}$ hemodialysis ${ }^{261}$ and surgery ${ }^{258}$ ) have been published and can form the basis for institutional policies and procedures. Health care professionals working in ICUs are obligated to be informed about prevention of infection associated with the procedures they perform and the devices with which they work daily.

\section{Intravascular Device-Related Bloodstream Infection}

\section{Impact}

Obtaining and maintaining reliable vascular access has become one of the most essential features of modern-day intensive care. Unfortunately, vascular access is associated with substantial and generally underappreciated potential for producing iatrogenic disease, particularly bloodstream infection (BSI) originating from infection of the percutaneous intravascular device (IVD) used for vascular accessIVD-related (IVDR) BSI, often referred to as "line sepsis." Nearly $60 \%$ of all nosocomial bacteremias derive from vascular access in some form ${ }^{17}$ and it is estimated that more than 500,000 IVD-related bloodstream infections occur in the United States each year. ${ }^{262,263}$ Studies performed a decade ago found that IVD-related BSIs are associated with excess attributable mortality ranging up to $35 \%{ }^{264}$; however, more recent case-control studies have not consistently found excess mortality, especially of this magnitude. ${ }^{265-267}$ This controversy aside, all studies examining the impact of IVD-related BSI on patient outcomes has found that IVD-related BSIs are associated with increased length of hospitalization and excess health care costs, averaging $\$ 30,000$ per case. ${ }^{264-267}$

IVD-related BSIs are largely preventable. The goal must not be simply to identify and treat these infections, but rather to prevent them. Over the past decade, much has been learned about the pathogenesis and epidemiology of infections associated with IVDs. By drawing on existent knowledge of the pathogenesis and epidemiology of IVDrelated BSI, rational and effective guidelines for prevention can be formulated.

\section{Definitions}

IVDs are associated with both local and systemic infection. The CDC has published definitions for IVD-related infection (see Box 51-1). ${ }^{16}$ These definitions are useful for the purposes of surveillance but rely heavily on the construct, central venous catheter-associated BSI, which implicitly assumes that each primary BSI (i.e., a BSI without an identifiable local infection) originates from a central venous catheter (CVC). This practice results in an over-estimation of the true risk of CVC-related infection because not all primary BSIs originate from a central venous device; some are secondary BSIs deriving from unrecognized postoperative surgical site or intra-abdominal infections or nosocomial pneumonias or originate from other vascular devices such as peripheral venous catheters or arterial catheters used for hemodynamic monitoring.

By applying molecular subtyping techniques ${ }^{107,268,269}$ to the results of semiquantitative or quantitative cultures of the removed IVD and blood cultures or the results of cultures of blood drawn through the IVD and a separate concomitant percutaneous peripheral blood culture, it is now possible to reliably determine whether an IVD was the source of a nosocomial BSI. Using these new diagnostic techniques allows formulation of simple but more rigorous definitions for IVD-related infection (Table 51-8), which we believe bear consideration as the standard for randomized trials and epidemiologic studies of IVDrelated infection. ${ }^{17}$

\section{Recognition and Diagnosis}

\section{Clinical Features}

Recent evidence-based guidelines provide the best current information on the evaluation of the ICU patient with fever or other signs of sepsis. ${ }^{270}$ Before any decision regarding initiation of antimicrobial therapy or removal of an IVD, the patient must be thoroughly examined to identify all plausible sites of infection including VAP, catheter-associated urinary tract infection, surgical site infection, antibiotic-associated colitis, and line sepsis. 


\begin{tabular}{|c|c|}
\hline IVD colonization & $\begin{array}{l}\text { (i) A positive semiquantitative* (or quantitative }{ }^{\dagger} \text { ) culture of the implanted portion or portions of the IVD; } \\
\text { (ii) absence of signs of local or systemic infection. }\end{array}$ \\
\hline Local IVD infection & $\begin{array}{l}\text { (i) A positive semiquantitative* (or quantitative }{ }^{\dagger} \text { ) culture of the removed IVD or a positive microscopic } \\
\text { examination or culture of pus or thrombus from the cannulated vessel; (ii) clinical evidence of infection } \\
\text { of the insertion site (i.e., erythema, induration or purulence); but (iii) absence of systemic signs of } \\
\text { infection and negative blood cultures, if done. }\end{array}$ \\
\hline IVDR BSI & $\begin{array}{l}\text { If the IVD is removed: } \\
\text { (i) A positive semiquantitative* (or quantitative }{ }^{\dagger} \text { ) culture of the IVD or a positive culture of the catheter } \\
\text { hub or infusate (or positive microscopic examination or culture of pus or thrombus from the } \\
\text { cannulated vessel) and one or more positive blood cultures, ideally percutaneously drawn, concordant } \\
\text { for the same species, ideally by molecular subtyping methods; (ii) clinical and microbiologic data } \\
\text { disclose no other clear-cut source for the BSI. } \\
\text { If the IVD is retained: } \\
\text { (i) If quantitative blood cultures are available, cultures drawn both from the suspect IVD and a peripheral } \\
\text { vein (or another IVD) are both positive and show a marked step-up in quantitative positivity ( } \geq \text { fivefold) } \\
\text { in the IVD-drawn culture; (ii) clinical and microbiologic data disclose no other clear-cut source for the } \\
\text { BSI. } \\
\text { or } \\
\text { (i) If automated monitoring of incubating blood cultures is available, blood cultures drawn concomitantly } \\
\text { from the suspect IVD and a peripheral vein (or another IVD) show both are positive, but the IVD- } \\
\text { drawn blood culture turns positive more than } 2 \text { hr before the peripherally drawn culture; (ii) clinical } \\
\text { and microbiologic data disclose no other clear-cut source for the BSI. }\end{array}$ \\
\hline
\end{tabular}

Table 51-9. Clinical, Epidemiologic, and Microbiologic Features of Intravascular Device-Related Bloodstream Infection

\begin{tabular}{|c|c|}
\hline Nonspecific & Suggestive of Device-Related Etiology \\
\hline $\begin{array}{l}\text { Fever } \\
\text { Chills, shaking rigors* } \\
\text { Hypotension, shock*} \\
\text { Hyperventilation, respiratory failure } \\
\text { Gastrointestinal* } \\
\text { Abdominal pain } \\
\text { Vomiting } \\
\text { Diarrhea } \\
\text { Neurologic*} \\
\text { Confusion } \\
\text { Seizures }\end{array}$ & $\begin{array}{l}\text { Patient unlikely candidate for sepsis (e.g., young, no underlying diseases) } \\
\text { Source of sepsis inapparent, no identifiable local infection } \\
\text { Intravascular device in place, especially central venous catheter } \\
\text { Inflammation or purulence at insertion site } \\
\text { Abrupt onset, associated with shock } \\
\text { Bloodstream infection caused by staphylococci (especially coagulase-negative } \\
\text { staphylococci), Corynebacterium spp., Candida, Trichophyton, Fusarium, or Malassezia } \\
\text { species }{ }^{\dagger} \\
\text { Very high-grade ( }>25 \mathrm{CFU} / \mathrm{mL} \text { ) candidemia } \\
\text { Cluster of cryptogenic infusion-associated bloodstream infections caused by Enterobacter } \\
\text { cloacae, Pantoea agglomerans, or Serratia marcescens } \\
\text { Sepsis refractory to antimicrobial therapy or dramatic improvement with removal of } \\
\text { cannula and infusion* }\end{array}$ \\
\hline
\end{tabular}

Despite the challenge of identifying the source of a patient's signs of sepsis, ${ }^{270}$ several clinical, epidemiologic, and microbiologic findings point strongly toward an IVD as the source of a septic episode (Table 51-9). ${ }^{262,271}$ Patients with an abrupt onset of signs and symptoms of sepsis without any identifiable local infection such as pneumonia or surgical site infection should prompt suspicion of infection of an IVD. The presence of inflammation or purulence at the catheter insertion site is now uncommon in patients with IVD-related BSI. ${ }^{272}$ However, if inflammation, especially any purulence, is seen in combination with signs and symptoms of sepsis, it is highly likely the patient has IVDrelated BSI and should prompt removal of the device. Finally, recovery of certain microorganisms in multiple blood cultures, such as staphylococci, Corynebacterium or Bacillus species, or Candida or Malassezia strongly suggests infection of an IVD.

\section{Blood Cultures}

Starting anti-infective drugs for suspected or presumed infection in the critically ill patient without first obtaining blood cultures from two separate sites, at least one of 
which is drawn from a peripheral vein by percutaneous venipuncture, is indefensible. The volume of blood cultured is essential to maximize the sensitivity of blood cultures for diagnosis of bacteremia or candidemia: in adults, obtaining at least $20 \mathrm{~mL}$, ideally $30 \mathrm{~mL}$, per drawing (each specimen containing $10 \mathrm{~mL}$ or $15 \mathrm{~mL}$, inoculated into aerobic and anaerobic media) significantly improves the yield as compared with obtaining only $5 \mathrm{~mL}$ at each drawing and culturing a smaller total volume. ${ }^{273,274}$ In adults, if at least $30 \mathrm{~mL}$ of blood is cultured, $99 \%$ of detectable bacteremias should be identified. ${ }^{273,275}$ Similar operating characteristics are achieved in the pediatric population using a weight-based graduated volume approach to blood cultures. ${ }^{276}$ Standard blood cultures drawn through CVCs provide excellent sensitivity for diagnosis of BSI but are less specific than cultures obtained from a peripheral vein. ${ }^{277,278}$ If the patient has a long-term multilumen catheter, it may be reasonable to obtain a specimen from each lumen of the catheter because studies have found discordance $(\approx 30 \%)$ among cultures obtained from different lumens of the same catheter. ${ }^{279}$

Every effort must be made to prevent introduced contamination when drawing blood cultures because a single contaminated blood culture has been shown to prolong hospitalization by 4 days and increase the costs of hospitalization by $\$ 4100$ to $\$ 4400 .{ }^{280,281}$ Tincture of iodine, isopropyl alcohol, chlorhexidine, or povidone-iodine combined with alcohol rather than povidone-iodine alone should be used for skin antisepsis prior to venipuncture for blood cultures, recognizing that studies have shown significantly reduced rates of contamination with use of these agents. ${ }^{200,281,282} \mathrm{Up}$ to $30 \%$ of blood cultures positive for coagulase-negative staphylococcus (CNS) represent true infection ${ }^{283,284}$; however, the majority of single positive cultures represent contamination, ${ }^{284}$ a finding that should reemphasize the need to obtain cultures from two separate sites whenever BSI is suspected.

\section{Cultures of Removed Intravascular Devices}

Removal and direct culture of the IVD has historically been the gold standard for confirming the presence of IVD-related BSI, particularly with short-term IVDs. Studies have shown that culturing catheter segments semiquantitatively on solid media ${ }^{285}$ or quantitatively in liquid media (e.g., removing the adherent organisms by sonication ${ }^{286}$ ) provides superior sensitivity and specificity for diagnosis of IVD-related BSI, with a strong correlation between high colony counts and line sepsis. Growth of greater than or equal to $15 \mathrm{CFUs}$ from a catheter segment by semiquantitative culture or growth of greater than or equal to $10^{3} \mathrm{CFUs}$ from a catheter cultured after sonication with accompanying local inflammation or signs of sepsis indicates local catheter infection. Significant growth in the absence of local or systemic inflammation suggests colonization of the device; if continued vascular access is necessary, a new device should be placed in a new location rather than replacing it with a new one in the same location by guidewire exchange.

Although recent studies ${ }^{287}$ have suggested that quantitative methods (e.g., sonication) are superior to the semi- quantitative methods (e.g., roll plate), other studies have shown them to be equivalent. ${ }^{288,289}$ Because hub contamination progressing to intraluminal colonization is the primary route of infection for long-term devices (e.g., devices in place $>10$ days), quantitative techniques may be superior to semiquantitative techniques in detecting infections from these types of devices because they remove organisms from both the internal and external surface of catheters. ${ }^{289}$ In contrast, semiquantitative methods may be preferred over quantitative methods in cases of suspected infection related to a short-term device (e.g., devices in place $<10$ days) because the primary route of infection in this setting is caused by extraluminal ingress of skin organisms at the catheter insertion site and the semiquantitative method is simple, less expensive, and allows identification of the infecting organisms a day earlier.

Direct and impression Gram stains ${ }^{290}$ or acridine orange stains $^{289}$ of intravascular segments of removed catheters have shown excellent correlation with quantitative techniques for culturing catheters and can permit rapid diagnosis of catheter-related infection.

To rigorously identify the mechanism of IVD-related BSI in prospective studies, it is necessary to culture all potential sources of microorganisms at the time of catheter removal (Fig. 51-8): skin of the insertion site, each catheter hub, infusate from each lumen, as well as implanted catheter segments. If the results of these cultures appear to link a BSI with microorganisms isolated from one or more portions of the device by phenotypic criteria, efforts then need to be made to conclusively establish concordance, beyond speciation and antimicrobial susceptibility pattern, using one or more molecular subtyping systems such as multi-locus enzyme electrophoresis, plasmid profile, or restriction-enzyme digestion of genomic DNA analyzed by pulsed-field electrophoresis. ${ }^{268,269,274,291}$

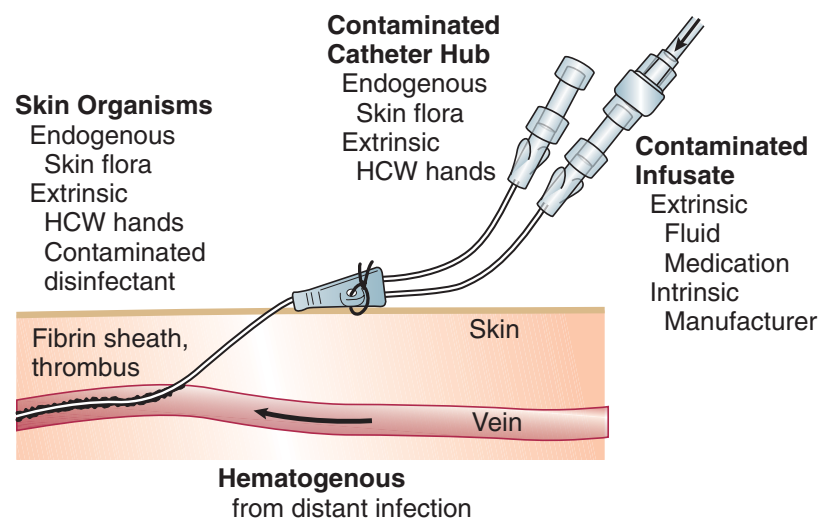

Figure 51-8. Potential sources of infection of a percutaneous IVD: the contiguous skin flora, contamination of the catheter hub and lumen, contamination of infusate, and

hematogenous colonization of the IVD from distant, unrelated sites of infection. HCW, health care worker. (From Crnich Cl, Maki DG: The promise of novel technology for the prevention of intravascular device-related bloodstream infection. I. Pathogenesis and short-term devices. Clin Infect Dis 2002;34:1232-1242.) 


\section{Diagnosis of Infection with Implanted Long-Term} Intravascular Devices

The methods described earlier require removal of the device for confirmation of IVD-related BSI. This can pose formidable challenges to management with long-term, surgically implanted IVDs such as Hickman and Broviac catheters, cuffed and tunneled hemodialysis catheters, and subcutaneous central venous ports. Only $15 \%$ to $45 \%$ of long-term IVDs that are removed for suspected infection are truly colonized or infected at the time of removal. ${ }^{292-295}$ To avoid unnecessary removal of IVDs, methods have been developed to diagnose IVD-related BSI while allowing the device to remain in place: (1) paired quantitative blood cultures drawn from the IVD and percutaneously from a peripheral vein ${ }^{289}$ and (2) differential time to positivity (DTP) of paired standard blood cultures, one drawn from the IVD and the other from a peripheral vein. ${ }^{296}$

If a laboratory has available an automated quantitative system for culturing blood (e.g., Isolator lysiscentrifugation system, Wampole Laboratories, Cranbury, $\mathrm{NJ}$ ), quantitative blood cultures drawn through the IVD and concomitantly by venipuncture from a peripheral vein (or another IVD) can permit the diagnosis of IVD-related bacteremia or fungemia to be made with sensitivity and specificity in the range of $80 \%$ to $95 \%,{ }^{289}$ without removal of the catheter, if empiric antimicrobial therapy has not yet been initiated. IVD-drawn cultures demonstrating 5- to 10-fold higher concentrations of microorganisms per milliliter, as compared with counts of the same micro-organism obtained in a culture drawn from a peripheral vein, confirm the presence of IVD-related BSI.
The differential-time-to-positivity (DTP) of paired blood cultures, one drawn through the IVD and the second, concomitantly from a peripheral vein, has also been shown to reliably identify IVD-related BSI of longterm IVDs if the blood culture drawn from the IVD turns positive 2 or more hours before the culture drawn peripherally. In studies of patients with long-term IVDs, the sensitivity and specificity of DTP ranged from $82 \%$ to $94 \%$ and $88 \%$ to $91 \%$, respectively. ${ }^{289,296}$ The performance of DTP in short-term IVDs has recently been examined, with disappointing results, ${ }^{297}$ a finding that is not entirely unexpected given the predominant extraluminal route of infection with these devices.

\section{Detection of Contaminated Infusate}

To diagnose infection caused by contaminated infusate, a sample of IV fluid, aspirated from the line, should be cultured quantitatively and qualitatively ${ }^{285}$; concordance with positive peripheral blood cultures, without another identifiable source for the patient's BSI, definitively implicates infected infusate as the cause of the BSI. Anaerobic culture techniques are not necessary unless blood or another biologic product is involved.

\section{Incidence}

Prospective studies, in which every attempt was made to conclusively identify the presence of an IVD-related BSI, show that every type of IVD carries some risk of causing BSI; however, the magnitude of risk varies greatly, depending on the type of device (Table 51-10). ${ }^{298}$ The device that poses the greatest risk of IVD-related BSI today is the

\begin{tabular}{|c|c|c|c|c|c|c|c|c|}
\hline \multirow[b]{3}{*}{ Device } & \multirow[b]{3}{*}{$\begin{array}{l}\text { Studies, } \\
n\end{array}$} & \multirow[b]{3}{*}{$\begin{array}{l}\text { Catheters, } \\
n\end{array}$} & \multirow[b]{3}{*}{$\begin{array}{l}\text { IVD-days, } \\
n\end{array}$} & \multirow[b]{3}{*}{$\begin{array}{l}\text { BSIs, } \\
n\end{array}$} & \multicolumn{4}{|c|}{$\begin{array}{c}\text { Rates of IVD-Related Bloodstream } \\
\text { Infection }\end{array}$} \\
\hline & & & & & \multicolumn{2}{|c|}{ Per 100 Devices } & \multicolumn{2}{|c|}{$\begin{array}{l}\text { Per } 1000 \\
\text { IVD-days }\end{array}$} \\
\hline & & & & & $\begin{array}{l}\text { Pooled } \\
\text { mean }\end{array}$ & $95 \% \mathrm{Cl}$ & $\begin{array}{l}\text { Pooled } \\
\text { mean }\end{array}$ & $95 \% \mathrm{Cl}$ \\
\hline Peripheral IV catheters & 11 & 10,910 & 28,720 & 13 & 0.1 & $0.1-0.2$ & 0.5 & $0.2-0.7$ \\
\hline Arterial catheters & 14 & 4366 & 21,397 & 37 & 0.8 & $0.6-1.1$ & 1.7 & $1.2-2.3$ \\
\hline $\begin{array}{l}\text { Short-term, nonmedicated } \\
\text { central venous catheters }\end{array}$ & 79 & 20,226 & 322,283 & 883 & 4.4 & 4.1-4.6 & 2.7 & $2.6-2.9$ \\
\hline Pulmonary artery catheters & 13 & 2057 & 8143 & 30 & 1.5 & $0.9-2.0$ & 3.7 & $2.4-5$ \\
\hline \multicolumn{9}{|l|}{ Hemodialysis catheters: } \\
\hline Temporary, noncuffed & 16 & 3066 & 51,840 & 246 & 8 & $7-9$ & 4.8 & $4.2-5.3$ \\
\hline $\begin{array}{l}\text { Long-term, cuffed, and } \\
\text { tunneled }\end{array}$ & 16 & 2806 & 373,563 & 596 & 21.2 & $19.7-22.8$ & 1.6 & $1.5-1.7$ \\
\hline $\begin{array}{l}\text { Peripherally inserted central } \\
\text { catheters (PICCS): }\end{array}$ & 15 & 3566 & 105,839 & 112 & 3.1 & $2.6-3.7$ & 1.1 & $0.9-1.3$ \\
\hline $\begin{array}{l}\text { Long-term tunneled and } \\
\text { cuffed central venous } \\
\text { catheters }\end{array}$ & 29 & 4512 & 622,535 & 1013 & 22.5 & $21.2-23.7$ & 1.6 & $1.5-1.7$ \\
\hline Subcutaneous venous ports & 14 & 3007 & 983,480 & 81 & 3.6 & $2.9-4.3$ & 0.1 & $0-0.1$ \\
\hline
\end{tabular}


CVC in its many forms (see Table 51-10): short-term, noncuffed, single-lumen or multilumen catheters inserted percutaneously into the subclavian or internal jugular vein have shown rates of catheter-related BSI in the range of $3 \%$ to $5 \%$ ( 2 to 3 per 1000 IVD-days) ${ }^{298}$ Far lower rates of infection have been encountered with surgically implanted cuffed Hickman or Broviac catheters and subcutaneous central venous ports $(1$ and 0.2 per 1000 IVD-days, respectively). ${ }^{298}$ Contrary to popular belief, peripherally inserted central catheters (PICCs) used in inpatients and arterial catheters are associated with rates of catheter-related BSI approaching those seen with short-term, noncuffed, and nontunnelled, multilumen CVCs-up to $2.1^{299}$ and $3.4^{300}$ BSIs per 1000 IVDdays, respectively.

\section{Pathogenesis and Risk Factors}

Two major sources of IVD-related BSI exist: (1) colonization of the IVD, catheter-related infection and (2) contamination of the fluid administered through the device, infusate-related infection. ${ }^{262}$ Contaminated infusate is the cause of most epidemic IVD-related BSIs; in contrast, catheter-related infections are responsible for most endemic IVD-related BSIs. ${ }^{17}$

In order for microorganisms to cause catheter-related infection, they must first gain access to the extraluminal or intraluminal surface of the device, where they can adhere and become incorporated into a biofilm that allows sustained infection and hematogenous dissemination. ${ }^{301}$ Microorganisms gain access to the bloodstream by one of three mechanisms (see Fig. 51-8): (1) skin organisms invade the percutaneous tract, probably assisted by capillary action, at the time of insertion or in the days following; (2) microorganisms contaminate the catheter hub (and lumen) when the catheter is inserted over a percutaneous guidewire or later manipulated; or (3) organisms are carried hematogenously to the implanted IVD from remote sources of local infection such as pneumonia.

With short-term IVDs (e.g., in place $<10$ days) such as peripheral IV catheters; arterial catheters; and noncuffed, nontunneled CVCs, most device-related BSIs are of cutaneous origin, from the insertion site, and gain access extraluminally, occasionally intraluminally at insertion with the guidewire. ${ }^{302,303}$ In contrast, contamination of the catheter hub and luminal fluid is the predominant mode of invasive infection with long-term IVDs (e.g., in place $>10$ days) such as cuffed Hickman- and Broviac-type catheters, subcutaneous central ports, and PICCs. ${ }^{304,305}$

Also important is recognizing that infusate (parenteral fluid, blood products, or IV medications) administered through an IVD can also occasionally become contaminated and produce device-related BSI. Contaminated fluid is fortunately an infrequent cause of endemic infusionrelated infection with most short-term IVDs; it is, however, an important cause of BSIs with arterial catheters used for hemodynamic monitoring and long-term IVDs such as Hickman or Broviac catheters, cuffed hemodialysis CVCs, and subcutaneous central venous ports. ${ }^{303,306,307}$

Most nosocomial epidemics of infusion-related BSI have been traced to contamination of infusate by gramnegative bacilli, introduced during its manufacture (intrin- sic contamination) or during its preparation and administration in the hospital (extrinsic contamination). ${ }^{143,308}$ If an epidemic is suspected, the epidemiologic approach must be methodical and thorough yet expeditious, directed toward establishing the bona fide nature of the putative epidemic infections (i.e., ruling out "pseudoinfections") ${ }^{242}$ and confirming the existence of an epidemic; defining the reservoirs and modes of transmission of the epidemic pathogens; and, most importantly, controlling the epidemic, quickly and completely. Control measures are predicated on accurate delineation of the epidemiology of the epidemic pathogen. The essential steps in dealing with a suspected nosocomial outbreak have recently been reviewed (and are discussed later). ${ }^{262}$

In recent years the factors associated with an increased risk of IVD-related BSI have become better delineated (Table 51-11). Prolonged hospitalization and severity of illness clearly influence the risk, and clinical states such as granulocytopenia, AIDS, and bone marrow transplanta-

\section{Table 51-11. Risk Factors for Intravascular}

Device-Related Bloodstream Infection with Short-Term Intravascular Devices

\begin{tabular}{|ll|} 
& $\begin{array}{l}\text { Relative Risk or } \\
\text { Odds Ratio }\end{array}$ \\
\hline Underlying Disease: & \\
AIDS (2) & 4.8 \\
Neutropenia (2) & $1-15.1$ \\
Gl disease (1) & 2.4 \\
Surgical service (1) & 4.4 \\
ICU/CCU placement (3) & $0.4-6.7$ \\
Extended hospitalization (3) & $1-6.7$ \\
Other intravascular devices (2) & $1-3.8$ \\
Systemic antibiotics (3) & $0.1-0.5$ \\
Active infection at another site (2) & $8.7-9.2$ \\
High APACHE III score (1) & 4.2 \\
Mechanical ventilation (1) & $2-2.5$ \\
Transplant patient (1) & 2.6 \\
Features of Insertion: & \\
Difficult insertion (1) & 5.4 \\
Maximal sterile barriers (1) & 0.2 \\
Tunneling (2) & $0.3-1$ \\
Insertion over a guidewire (8) & $1-3.3$ \\
\hline Insertion Site: & \\
Internal jugular vein (6) & $1-3.3$ \\
Subclavian vein (5) & $0.4-1$ \\
Femoral vein (2) & $3.3-4.8$ \\
Defatting insertion site (1) & 1 \\
Use a multilumen catheter (8) & -6.5 \\
Catheter Management: & \\
Routine change of IV set (2) & 1 \\
Staffing in SICU (nurse-to-patient ratio) (1) & \\
$1: 2$ & 61.5 \\
$1: 1.5$ & 15.6 \\
$1: 1.28$ & 4 \\
$1: 1$ & 5.3 \\
Inappropriate catheter usage (1) & $1-8.7$ \\
Duration of catheterization >7 days (5) & $17.9-44.1$ \\
Colonization of catheter hub (3) & -4.8 \\
Parenteral nutrition (2) & \\
\hline Modified from Safdar NS, Kluger DM, Maki DG: A review of risk factors \\
for catheter-related infection caused by percutaneously inserted, \\
noncuffed central venous catheters: Implications for preventive \\
strategies. Medicine 2002;81:466-479. & \\
\hline
\end{tabular}


tion have been associated with fourfold to sixfold increased rates of IVD-related BSI. ${ }^{309,310}$ However, the features of the IVD, its insertion, and its maintenance appear to have far greater impact on the overall risk of infection. In 289 patients, Merrer and colleagues ${ }^{311}$ found that insertion of an IVD in the femoral versus the subclavian vein was associated with a greatly increased risk of infection (20 versus 3.7 BSIs per 1000 IVD-days, $P<.001$ ) and thrombotic complications $(21.5 \%$ versus $1.9 \%, P<.001) .311$ Moreover, Robert and colleagues ${ }^{312}$ found that patients with primary BSI were more likely to have received care during times when there was a lower nursing-to-patient ratio and a higher proportion of temporary ("float") nurses rather than the fulltime nursing staff. ${ }^{312}$

\section{Microbiology}

Figure 51-9 summarizes the microbial profile of IVDrelated BSI from 159 published prospective studies. ${ }^{313}$ As might be expected from knowledge of the pathogenesis of these infections, skin microorganisms account for the largest proportion of these infections.

\section{Strategies for Prevention}

Recommendations for the prevention of IVD-related BSIs were published by the Hospital Infection Control Practices Advisory Committee (HICPAC) several years ago. ${ }^{129}$ Table 51-12 summarizes the recommendations of the 2001 HICPAC guideline for the prevention of IVD-related BSI and scores each recommendation on the basis of the quality of the available scientific evidence. It must be reaffirmed that measures for prevention of any nosocomial infection must, wherever possible, be based on the best understanding of pathophysiology and epidemiology and, whenever possible, controlled clinical trials.

\section{At-Device Insertion}

1. Choice of catheter and site of device insertion: Obviously, the choice of IVD inserted into a patient will be guided primarily by that patient's particular needs (e.g., hemodialysis versus fluid administration). However, the astute clinician can mitigate much of the risk associated with vascular access by choosing the best device for the task at hand and inserting the IVD in a location associated with the least risk of infection. Studies suggest that multilumen IVDs are associated with a higher risk of infection than single-lumen catheters. ${ }^{314}$ That said, if a patient has need for multiple infusions, inserting several single lumen catheters will pose greater risks than a single multilumen catheter.

To date, there have been no randomized studies designed to evaluate the optimal location for placement of short-term CVCs. However, the data accumulated from numerous observational studies suggest that the lowest risk of IVD-related BSI is seen with subclavian vein insertion and the highest risk with femoral vein insertion, with an intermediate level of risk associated with jugular vein insertions. ${ }^{303,311}$

The femoral vein is often used for central venous access, especially on nonsurgical services, because of the ease of cannulation and the lower risk of mechanical complications from insertion (i.e., bleeding or pneumothorax). Unfortunately, prospective studies evaluating the risk of femoral vein device placement have shown that CVCs placed in the femoral vein are more likely to be colonized at the time of removal than catheters placed in the internal jugular vein $(R R=4.7$, $\mathrm{CI}=2$ to $8.8, P=.0001)^{315}$ and are associated with an increased risk of IVD-related BSI when compared with CVCs placed in the subclavian vein $(4.4 \%$ versus $1.5 \%, P=.07){ }^{311}$ Furthermore, recent prospective studies have found higher rates of catheter-related deep vein thrombosis with femoral catheters, in the range of $7 \%$ to $25 \% .^{310,311}$ In general, we believe femoral access should be used only if emergent access is required, the inexperience of the operator limits placement in the upper body, or there is a contraindication to placement in the upper body (no available sites, an extensive burn, or severe coagulopathy). If a shortterm CVC must be placed in the femoral vein or artery, we believe it is important that the catheter insertion site be located at least 2 inches $(5 \mathrm{~cm})$ below the inguinal crease or an intertriginous area, which is heavily colonized with bowel organisms and yeasts; this also allows a more secure protective dressing to be affixed.

In contrast to short-term CVCs, observational studies of hemodialysis catheters have not been able to confirm a lower rate of infection with catheters inserted in the subclavian vein as compared with those inserted in the internal jugular vein, ${ }^{316}$ although there is still excess risk associated with femoral vein placement. ${ }^{317}$ More importantly, prospective studies of catheters used for hemodialysis have demonstrated a significant risk of great vein thrombosis and stenosis in catheters inserted

\begin{tabular}{rl|l} 
Coagulase-negative staphylococci & $31 \%$ \\
\cline { 2 - 2 } Staphylococcus aureus & \multicolumn{1}{|c|}{$18 \%$} \\
\cline { 2 - 2 } Enteric gram-negative bacilli & \multicolumn{1}{|c|}{$14 \%$} \\
\cline { 2 - 2 } Pseudomonas aeruginosa & \multicolumn{2}{|c|}{$8 \%$} \\
\cline { 2 - 2 } Candida spp. & $6 \%$ \\
\cline { 2 - 2 } Corynebacterium spp. & $5 \%$ \\
Enterococcus spp. & $4 \%$ \\
\cline { 2 - 2 } & & \\
\cline { 2 - 2 } & &
\end{tabular}

Figure 51-9. Microbial profile of intravascular device-related bloodstream infection based on an analysis of 159 published prospective studies. (Modified from Maki DG, Kluger DM, Crnich C): The microbiology of intravascular device-related (IVDR) infection in adults: An analysis of 159 prospective studies and implications for prevention and treatment. In Abstracts and Proceedings from the 40th Annual Meeting of the Infectious Disease Society of America. Chicago, Infectious Disease Society of America, 2002.) 
Table 51-12. Summary of CDC/HICPAC Guideline for Prevention of IVD-Related Bloodstream Infection

Recommendation

Strength of Evidence*

General measures

- Educate all health care workers involved with IVD care and maintenance

- Ensure adequate nursing staffing levels in ICUs

IB

Surveillance

- Monitor institutional IVD infection rates of IVD-related BSI

- Express rates of CVC-related BSI per 1000 CVC-days

IA

At-catheter insertion

- Aseptic technique:

Hygienic hand care before insertion or manipulation of any IVD

Clean or sterile gloves during insertion and manipulation of noncentral IVDs

Maximal barrier precautions during insertion of CVCs: mask, cap, sterile gown, gloves, drapes

- Dedicated IVD team strongly recommended

- Cutaneous antisepsis: first choice, chlorhexidine; however, tincture of iodine, an iodophor, or $70 \%$ alcohol are acceptable (no recommendations for use of chlorhexidine in infants younger than 2 months, unresolved issue)

- In adults, other than hemodialysis catheters (jugular site preference), use a subclavian site rather than a jugular or femoral site for CVC access (in pediatric patients, no recommendations for preferred site, unresolved issue)

- Use of sutureless securement device

Sterile gauze or a semipermeable polyurethane dressing to cover site

- No systemic or topical antibiotics at insertion

Maintenance

- Remove IVD as soon as no longer required

Monitor IVD site daily

- Change dressing of CVC insertion site at least weekly

- Do not use topical antibiotic ointments

- Change needless IV systems at least as frequently as the administration set; replace caps no more frequently than every 3 days or per manufacturer's recommendations

- Complete lipid infusions within $12 \mathrm{hr}$

- Replace administration sets no more frequently than every $72 \mathrm{hr}$. When lipid-containing admixtures or blood products are given, sets should be replaced every $24 \mathrm{hr}$; with propofol, every 6-12 hr

- Replace peripheral IVs every $72-96 \mathrm{hr}$

- Do not routinely replace CVCs or PICCs solely for prevention of infection

- Do not remove CVCs or PICCs solely because of fever unless IVD infection is suspected, but replace catheter if there is purulence at the exit site, especially if the patient is hemodynamically unstable and IVD-related BSI is suspected

Technology

- Use antimicrobial-coated or antiseptic-impregnated CVC in adult patients if institutional rate of BSI is high despite consistent application of preventive measures and catheter likely to remain in place $>5$ days (no data or recommendations for pediatric patients)

- Use chlorhexidine-impregnated sponge dressing for adolescent or adult patients with uncuffed CVCs or other catheters likely to remain in place $>5$ days (no recommendation for children, do not use in neonates younger than 7 days old or gestational age younger than 26 wk)

- Use prophylactic antibiotic lock solution only in patients with long-term IVDs who have continued to experience IVD-related BSIs despite consistent application of infection control practices

*Taken from CDC/HICPAC system of weighting recommendations based on scientific evidence. IA, strongly recommended for implementation and supported by well-designed experimental, clinical, or epidemiological studies. IB, strongly recommended for implementation and supported by some experimental, clinical, or epidemiological studies and a strong theoretical rationale. IC, required by state or federal regulations, rules, or standards. II, suggested for implementation and supported by suggestive clinical or epidemiological trials or a theoretical rationale. Unresolved issue, an unresolved issue for which evidence is insufficient or no consensus regarding efficacy exists. NR, no recommendation for or against at this time.

BSI, bloodstream infection; CVC, central venous catheter; ICU, intensive care unit; IV, intravenous; IVD, intravascular device.

Modified from O'Grady NP, Alexander M, Dellinger EP, et al: Guidelines for the prevention of intravascular catheter-related infections. Clin Infect Dis 2002; $35: 1281-1307$.

into the subclavian vein that approaches $40 \%$ to $50 \%$ as compared with rates of $0 \%$ to $10 \%$ with catheters inserted into the internal jugular vein. ${ }^{318,319}$ On the basis of these data, internal jugular vein insertion is preferable to subclavian vein insertion for central access for hemodialysis.
2. Barrier precautions: Hand hygiene with an antisepticcontaining preparation, either conventional handwashing with chlorhexidine ( $2 \%$ to $4 \%$ ) or with a waterless alcohol rub or gel, ${ }^{171}$ must always precede the insertion of an IVD and should also precede subsequent handling of the device or its administration set. ${ }^{129}$ A new 
pair of disposable, nonsterile gloves, using a "notouch" technique, is adequate for the placement of peripheral IV catheters in most patients; however, sterile gloves should be used during insertion in highrisk patients such as those with granulocytopenia. Sterile gloves are strongly recommended for placement of all other types of IVDs that are associated with a $1 \%$ or higher risk of associated bacteremia, specifically arterial catheters and all types of centrally placed devices including PICCs. ${ }^{129}$

Studies have shown that the use of maximal barriers including a long-sleeved, sterile surgical gown, mask, cap and large sterile drape, and sterile gloves significantly reduces the risk of CVC-related BSI (0.08 BSIs with maximal barriers versus 0.5 BSIs per 1000 IVDdays without maximal barriers, $P=.02) .{ }^{320}$ The use of maximal barriers has further been shown to be highly cost effective. ${ }^{320}$ Considering that of all IVDs, CVCs are most likely to produce nosocomial BSI, a strong case can be made for mandating maximal barrier precautions during the insertion of all central IVDs. ${ }^{129}$ They are not necessary, however, for arterial catheters used for hemodynamic monitoring, during which sterile gloves and a sterile fenestrated drape will suffice. ${ }^{321}$

3. IV teams: Good technique is also essential. Studies have shown that the use of special IV therapy teams, consisting of trained nurses or technicians who can assure a consistent and high level of aseptic technique during catheter insertion and in follow-up care of the catheter, have been associated with substantially lower rates of catheter-related BSI and are cost effective. ${ }^{322,323}$ But even if an institution does not have an IV team, it can greatly reduce its rate of IVD-related BSI by formal education of nurses and physicians and stringent adherence to IVD care protocols. ${ }^{324,325}$

4. Cutaneous antisepsis: Given the evidence for the importance of cutaneous microorganisms in the pathogenesis of short-term IVD-related infections, measures to reduce colonization of the insertion site would seem of the highest priority, particularly the choice of chemical antiseptics for disinfection of the site. Nine randomized, prospective trials comparing a chlorhexidine-containing antiseptic to either povidone-iodine or alcohol for preparation of the skin prior to insertion of a short-term IVD have been reported..$^{201-203,326}$ In the largest study to date, a randomized trial in 1050 CVCs and arterial catheters placed in a university hospital ICU, cutaneous antisepsis with $1 \%$ tincture of chlorhexidine showed a highly significant reduction in IVDrelated BSIs compared with an iodophor $(R R=0.35$, $P<.01) .{ }^{326}$ More recently, a meta-analysis that examined results from eight of the nine aforementioned studies found that use of chlorhexidine was associated with a nearly $50 \%$ reduction in the risk of IVD-related compared with povidone-iodine $(\mathrm{RR}=0.49,95 \%$ $\mathrm{CI}=0.28$ to 0.88$){ }^{201}$

\section{Insertion Site Care and IVD Maintenance}

1. IVD dressings: IVDs can be dressed with sterile gauze and tape or with a sterile transparent, semipermeable, polyurethane film dressing. The available data suggest that the two types of dressings are equivalent in terms of their impact on IVD-related BSI with peripheral IVs and short-term CVCs. ${ }^{327-329}$ In contrast, results from studies of arterial catheters have found that polyurethane dressings greatly increase the risk of IVD-related BSI. ${ }^{327,330}$ As a result, polyurethane dressings should probably not be used on arterial catheters until future studies confirm their safety.

2. Topical antimicrobial ointments: In theory, application of a topical antimicrobial agent to the catheter insertion site should confer some protection against microbial invasion. Clinical trials of a topical combination antibacterial ointment containing polymyxin, neomycin, and bacitracin with peripheral IVs have shown marginal benefit, ${ }^{331}$ but the use of polyantibiotic ointments has been associated with a fivefold increased frequency of Candida infection, limiting their utility. ${ }^{331,332}$

The topical antibacterial mupirocin, which is active primarily against gram-positive organisms, was shown in one study to significantly reduce colonization of internal jugular catheters without increasing colonization by Candida spp., ${ }^{333}$ and a more recent study by Sesso and colleagues ${ }^{334}$ showed significant reductions in hemodialysis catheter colonization (3.17 versus 14.27 per 1000 IVD-days, $P=<.001$ ) and $S$. aureus IVD-related BSIs (0.71 versus 8.92 BSIs per 1000 IVDdays, $P=<.001){ }^{334}$ Unfortunately, resistance of $S$. aureus $^{335}$ and coagulase-negative staphylococci ${ }^{336}$ rapidly emerges during wide-scale mupirocin use, ${ }^{337}$ which contravenes its use as a topical agent for the prevention of IVD-related BSI at this time. ${ }^{129}$

Three prospective studies of topical povidone-iodine ointment applied to central venous catheter sites have failed to show a statistical benefit to its use, ${ }^{331,338,339}$ but a single comparative trial in subclavian hemodialysis catheters showed that the use of topical povidoneiodine ointment was associated with a fourfold reduction in the incidence of IVD-related $S$. aureus BSI. ${ }^{340}$ Therefore if a topical agent is to be used with hemodialysis catheters, an iodophor may be most desirable.

3. Replacement of the device: Studies have shown that peripheral IVs may be safely left in place for up to 96 hours if the patient and the insertion site is monitored closely. ${ }^{341}$ Studies have suggested that the duration of peripheral catheterization may be prolonged even further, ${ }^{342}$ but, viewing reports of increasing nosocomial $S$. aureus bacteremias linked to prolonged peripheral venous catheterization, ${ }^{343}$ more studies are required before this can become considered acceptable routinely.

Scheduled replacement of short-term, noncuffed, nontunneled CVCs has long been practiced in many centers; however, some studies have called this practice into question. ${ }^{344}$ Moreover, a meta-analysis found no benefit to routine replacement of short-term CVCs. ${ }^{345}$ On the basis of these data, there appears to be no indication for scheduled replacement of short-term CVCs that are functioning well and show no clinical signs of infection. 
4. Guidewire exchanges of CVCs: The management of CVCs that must be replaced, either because of mechanical malfunction or suspected infection, deserves special attention. Replacement of CVCs by guidewire exchange is associated with a reduced risk of mechanical complications $^{344,345}$; however, it is also associated with an increased risk of the newly placed CVC becoming infected and causing CVC-related BSI. ${ }^{344}$ As a result, if circumstances necessitate guidewire exchange for placement of a new catheter (e.g., the patient has limited sites for access, is morbidly obese, or is at high risk of mechanical complications because of underlying coagulopathy), the same strict aseptic technique, which includes full barrier precautions, must be used. However, the tip and/or intracutaneous segment(s) of the removed CVC should routinely be sent for culture to determine whether the insertion tract is colonized. If it is, the newly inserted CVC should be promptly removed and a new CVC placed percutaneously in a new site. If the tract is not colonized, the newly exchanged CVC can remain in the old insertion site.

Although small studies have found some utility of guidewire exchange in the management of CVCs suspected of being infected, ${ }^{346,347}$ we believe that, in the absence of randomized studies demonstrating its safety, guidewire exchange generally should not be performed if there is suspicion of IVD-related BSI, especially if there are signs of local infection such as purulence or erythema at the insertion site or signs of systemic sepsis without a source. In these cases the old catheter should be removed and cultured, and a new catheter should be inserted in a new site.

5. Replacing the delivery system: Whereas most infusionrelated BSIs are caused by infection of the device used for vascular access, infusate can occasionally become contaminated and cause endemic BSIs. ${ }^{303,348}$ If an infusion runs continuously for an extended period, the cumulative risk of contamination increases, and there is further risk that contaminants can grow to concentrations that could produce BSI in the recipient of the fluid. For more than 25 years, most U.S. hospitals have routinely replaced the entire delivery system of patients' IV infusions at 24- or 48-hour intervals ${ }^{349}$ to reduce the risk of BSI from extrinsically contaminated fluid. Prospective studies indicate that IV delivery systems need not be replaced more frequently than every 72 to 96 hours, including infusions used for total parenteral nutrition or any infusions in ICU patients ${ }^{341,350}$; extending the duration of use can permit cost savings to hospitals. ${ }^{350}$

Four clinical settings might be regarded as exceptions to using 72 hours as an interval for routine set change $^{350}$ : (1) administration of blood products, (2) administration of lipid emulsion, (3) arterial pressure monitoring, and (4) suspicion of an epidemic of infusion-related BSI. In these circumstances, it may be most prudent for administration sets to be changed routinely at 24 - or 48 -hour intervals.
Arterial infusions used for hemodynamic monitoring appear to be more vulnerable to becoming contaminated during use and producing endemic ${ }^{348}$ or epidemic septicemia, ${ }^{96}$ caused by gram-negative bacilli. If the infusion for hemodynamic monitoring is set up so that the fluid flows continuously through the system, thus eliminating a blind stagnant column of fluid, extrinsic contamination appears to be greatly reduced and may even eliminate the need to replace the administration set, transducer assembly, and other components of the system at frequent intervals. ${ }^{351,352}$ If disposable transducers are used, there appears to be no need to replace the transducer assembly and other components of the delivery system more frequently than every 4 days, ${ }^{351}$ and it may be safe to replace them even less frequently. ${ }^{352}$

6. Anticoagulation: Thrombus formation on an intravascular device is associated with an increased risk of infection. ${ }^{353,354}$ Two prospective studies have been performed to examine the efficacy of warfarin anticoagulation for reducing rates of IVD-associated thrombosis with long-term IVDs. ${ }^{355,356}$ Both studies found that use of warfarin in a dose of $1 \mathrm{mg} /$ day was associated with significantly reduced rates of thrombosis with longterm IVDs, although no data were provided on rates of IVD-related BSI.

The use of prophylactic heparin for reducing rates of IVD-related thrombosis and infection has been evaluated in a meta-analysis. ${ }^{357}$ Examining a variety of different administration techniques in 14 randomized controlled studies, Randolph and colleagues ${ }^{357}$ concluded that systemic heparinization significantly reduced the risk of IVD-associated thrombosis $(\mathrm{RR}=0.43, \mathrm{CI}=0.23-0.78)$ and device colonization $(\mathrm{RR}=0.18, \mathrm{CI}=0.06$ to 0.6$)$ but failed to show a reduction in IVD-related BSIs. Heparin-bonded pulmonary artery catheters may be less prone to IVD-related BSI than nonheparinized catheters. ${ }^{303,358,359}$

On the basis of these studies, low-level anticoagulation with warfarin is warranted for long-term IVDs as long as there is no contraindication (bleeding diathesis, brain tumor, or predilection to falls) and the INR is maintained below 1.6. ${ }^{355}$ For short-term IVDs, the use of low-dose subcutaneous heparin is more appropriate; it is commonly given to patients with CVCs or arterial lines as part of ICU thromboembolism prophylaxis.

\section{Novel Technology}

Despite compliance with recommended guidelines, many centers continue to have high rates of IVD-related BSI. Novel technology holds much promise (Table 51-13). Innovative technologies designed to reduce the risk of IVD-related BSI have proved to be not only effective but also to reduce health care costs, both with short-term and long-term IVDs. ${ }^{301,360}$

1. Novel securement devices: Recently, a novel sutureless device for securing noncuffed vascular catheters has 
Table 51-13. Novel Technology for Prevention of IVD-Related Bloodstream Infection That Has Been Examined in Randomized Clinical Trials

\begin{tabular}{|c|}
\hline $\begin{array}{l}\text { Chlorhexidine For Cutaneous Antisepsis } \\
\text { Securement Devices } \\
\text { Topical anti-infective creams or ointments } \\
\text { Polymyxin, neomycin, bacitracin polyantibiotic ointment } \\
\text { Povidone-iodine ointment } \\
\text { Mupirocin ointment }\end{array}$ \\
\hline $\begin{array}{l}\text { Dressings } \\
\text { Transparent, polyurethane film dressings } \\
\text { Hyperpermeable polyurethane dressings } \\
\text { Hydrocolloid dressings } \\
\text { Chlorhexidine-impregnated sponge dressings }\end{array}$ \\
\hline $\begin{array}{l}\text { Innovative IVD Design } \\
\text { Cuffed and tunneled CVCs } \\
\text { Subcutaneous central venous ports } \\
\text { Attachable silver-impregnated cuffs } \\
\text { Peripherally inserted central venous catheters (PICCs) }\end{array}$ \\
\hline $\begin{array}{l}\text { Anti-Infective-Coated Catheters } \\
\text { Benzalkonium chloride-impregnated catheters } \\
\text { Chlorhexidine-silver sulfadiazine-coated catheters } \\
\text { Cefazolin-coated catheters } \\
\text { Minocycline-rifampin-coated catheters } \\
\text { Silver-impregnated catheters }\end{array}$ \\
\hline $\begin{array}{l}\text { Anti-Infective Catheter Hubs } \\
\text { lodinated chamber } \\
\text { External povidone-iodine-saturated sponge cap }\end{array}$ \\
\hline $\begin{array}{l}\text { Anti-Infective Lock Solutions for Long-Term IVDs } \\
\text { Gentamicin } \\
\text { Vancomycin } \\
\text { Vancomycin/ciprofloxacin } \\
\text { Trisodium citrate/gentamicin } \\
\text { Minocycline/ethylenediaminetetraacetic acid (EDTA) } \\
\text { Ethanol } \\
\text { Taurolidine }\end{array}$ \\
\hline Scheduled (Prophylactic) Thrombolysis with Urokinase \\
\hline $\begin{array}{l}\text { CVC, central venous catheter; IVD, intravascular device. } \\
\text { Modified from Crnich CJ, Maki DG: The promise of novel technology } \\
\text { for the prevention of intravascular device-related bloodstream } \\
\text { infection. I. Pathogenesis and short-term devices. Clin Infect Dis } \\
2002 ; 34: 1232-1242 \text { and } 1362-1368 \text {. }\end{array}$ \\
\hline
\end{tabular}

become available (StatLock, Venetec International). In a randomized trial of the device, premature loss of pediatric PICCs caused by accidental extrusion and PICC-associated thrombosis was significantly reduced, ${ }^{361}$ and in two additional trials the incidence of catheter-related BSI was significantly reduced with the use of the novel securement device, both in adults and children with PICCs. ${ }^{361,362}$

The promise of this device for reducing infection may derive from elimination of a festering skin suture wound contiguous to the newly inserted catheter and minimizing to-and-fro movement of the catheter, which may promote invasion of the tract by cutaneous microorganisms through capillary action. ${ }^{363}$

2. Novel dressings: Studies of polyurethane dressings, which contain antiseptics such as povidone-iodine or ionized silver, have been disappointing. However, on the basis of demonstrated superiority of chlorhexidine for cutaneous disinfection of access sites, a novel chlorhexidine-impregnated sponge dressing has been developed (Biopatch, Johnson and Johnson Medical, Inc.). It maintains a high concentration of the antiseptic on the insertion site under the dressing. The largest study to date found that use of the chlorhexidineimpregnated sponge dressing was associated with a $60 \%$ reduction in catheter-related $\mathrm{BSI}(\mathrm{RR}=0.37$, $P=.01){ }^{364}$ Although there were no adverse side effects associated with the use of this dressing in this trial in adults, a pediatric trial found that $15 \%$ of low-birth-weight neonates developed local dermatotoxicity. ${ }^{365}$

3. Anti-infective impregnated catheters: Intravascular devices directly coated or impregnated with antimicrobials or antiseptics have been intensively studied over the past decade. Eighteen randomized trials evaluating the efficacy of chlorhexidine-silver-sulfadiazine- or minocycline-rifampin-impregnated CVCs have been published in full article or abstract form since $1994 .{ }^{268,269,291,366,367}$

Of the 16 published studies that examined the effect of antimicrobial-impregnated CVCs on rates of CVCrelated BSI, 12 found either a statistically significant reduction or a strong trend toward a reduction in rates of CVC-related BSI. ${ }^{366,367}$ Aggregate analysis of the 15 studies that compared antimicrobial-impregnated CVCs with nonimpregnated CVCs, ${ }^{366,367}$ encompassing a total of 4250 CVCs, shows that antimicrobialimpregnated CVCs are associated with a $40 \%$ reduction in CVC-related BSI (61 BSIs/2129 devices versus 101 BSIs $/ 2118$ devices, OR $0.60,95 \% \mathrm{CI}=0.44$ to 0.82 , $P=.001$ ), a result remarkably similar to the findings of three published meta-analyses. ${ }^{301,368,369}$

Finally, two rigorous and sophisticated economic analyses have found that antimicrobial-impregnated CVCs are cost effective. ${ }^{370,371}$ Veenstra and colleagues showed thatantimicrobial-impregnatedCVCsremained cost effective even if the cost of a CVC-related BSI was as low as $\$ 687$ per case; cost savings were \$196 per antimicrobial-impregnated CVC when a more realistic cost of a CVC-related BSI of $\$ 9738$ was used in the analysis. ${ }^{370}$ Shorr and colleagues ${ }^{371}$ showed that use of antimicrobial-impregnated CVCs was associated with a cost savings of $\$ 9600$ per CVC-related BSI prevented and that $\$ 165$ to $\$ 280$ would be saved for every patient who received an antimicrobialimpregnated CVC.

On the basis of this large body of data, two national advisory panels have recommended the use of antimicrobial-impregnated CVCs in clinical settings where, despite rigorous application of other preventive interventions, rates of IVD-related BSI remain unacceptably high (i.e., $\geq 3.3$ BSIs per 1000 IVD-days). ${ }^{129,372}$

4. Antimicrobial lock solutions: Given the importance of hub contamination and intraluminal colonization in the genesis of IVD-related BSI with long-term IVDs, 
intraluminal instillation of an antibiotic or antiseptic solution has the potential to reduce the risk of BSI associated with these devices. Six randomized, prospective trials have examined a vancomycin-containing antibiotic lock solution for the prevention of IVDrelated BSI, the largest of which found that use of a vancomycin or vancomycin/ciprofloxacin lock solution reduced the risk of IVD-related BSI nearly $80 \%$ $(P=.005)$, with no evidence that the use of the lock solution promoted colonization or infection by vancomycin-resistant bacteria or fungi. ${ }^{373,374}$ Yet concern about the emergence of resistance with prophylactic antibiotic-containing lock solutions has limited their wider acceptance to date. However, the use of prophylactic antibiotic lock solution is considered acceptable in the 2001 HICPAC Guideline if a patient with an essential long-term IVD has continued to experience recurrent IVD-related BSIs despite consistent application of infection control practices. ${ }^{129}$

Various other prophylactic lock solutions have been studied as a means of preventing IVD-related BSI including trisodium citrate/gentamicin, ${ }^{375}$ minocycline/ ethylenediaminetetraacetic acid (EDTA) ${ }^{376}$ ethanol, ${ }^{377}$ and taurolidine-containing solutions. ${ }^{378}$ Concerns about increased IVD complication rates ${ }^{378}$ and drugrelated toxicity ${ }^{375}$ associated with the use of certain types of lock solutions, combined with the limited number of patients who have been studied while receiving these agents, precludes their routine use at this time.

5. Catheter hubs: A novel catheter hub that contains a chamber filled with iodinated alcohol has been shown to be effective in preventing colonization of IVDs in an animal model. ${ }^{379}$ Use of this same hub model in some clinical studies has demonstrated significantly lower rates of IVD colonization compared with IVDs with control hubs. ${ }^{380,381}$ One clinical trial has also demonstrated reduced rates of IVD-related BSIs with use of this hub ( $4 \%$ versus $16 \%, P<.01)$. A subsequent study also showed a reduction in hub-related IVDrelated BSIs $(1.7 \%$ versus $7 \%, P<.049)$, but overall rates of IVD-related BSIs in both groups were similar. ${ }^{381}$ Another study was unable to find any benefit with regards to IVD colonization or IVD-related BSI with use of the novel hub. ${ }^{382}$ This device is not yet available in the United States and until further studies more conclusively demonstrate its benefit, its use cannot be recommended at this time.

\section{Ventilator-Associated Pneumonia}

\section{Incidence and Impact}

Hospital-acquired pneumonia (HAP) is defined as pneumonia that develops more than 48 hours after hospitalization. ${ }^{383} \mathrm{VAP}$ is a subset of HAP and is defined as pneumonia that occurs more than 48 to 72 hours after initiating mechanical ventilation. ${ }^{383}$ Nearly 300,000 episodes of HAP occur in U.S. hospitals each year. ${ }^{384}$ More than $90 \%$ of HAPs occur in patients undergoing mechanical ventilation, and $10 \%$ to $20 \%$ of mechanically ventilated patients will develop VAP. ${ }^{385}$ VAP is the second most common nosocomial infection in U.S. ICUs participating in the National Nosocomial Infection Surveillance (NNIS) program with median rates of VAP ranging from 2.3 cases per 1000 ventilator-days in pediatric units to 11.4 cases per 1000 ventilator-days in trauma units (Table 51-14). ${ }^{133}$ VAP increases length of hospitalization by 6.1 days and health care costs by $\$ 10,019$ when compared with matched controls who had not developed VAP. ${ }^{385}$ More importantly, VAP is associated with more nosocomial deaths than is infection at any other site ${ }^{386}$-at least 50,000 deaths in U.S. centers annually - and increases hospital mortality at least twofold in affected individuals. ${ }^{385}$

\section{Pathogenesis}

In the normal nonsmoking host, multiple host defense mechanisms contribute to protection against pneumonia. ${ }^{387}$ The respiratory tract above the vocal cords is normally heavily colonized by bacteria, but unless the person has chronic bronchitis or has had respiratory tract instrumentation, the lower respiratory tract is normally sterile; although healthy adults aspirate frequently during sleep, the lower airways and pulmonary parenchyma of healthy, nonsmoking persons without lung disease are remarkably free of microbial colonization. ${ }^{388}$ The major defense mechanisms include anatomic airway barriers, the cough reflex, mucus ${ }^{389}$ and mucociliary clearance. ${ }^{390}$ Below the terminal bronchioles, the cellular and humoral immune systems are essential components of host defense. ${ }^{391}$ Alveolar macrophages and leukocytes remove particulate matter and potential pathogens, elaborate cytokines that activate the systemic cellular immune response and act as antigenpresenting cells to the humoral arm of immunity. ${ }^{392}$ Immunoglobulins and complement opsonize bacteria and bacterial products within the respiratory tract, assisting phagocytosis.

In the mechanically ventilated patient, numerous factors conspire to compromise host defenses: Critical illness, comorbidities, and malnutrition impair the immune system. ${ }^{393,394}$ Endotracheal intubation thwarts the cough reflex; compromises mucociliary clearance; injures the tracheal epithelial surface; and provides a direct conduit for bacteria from the mouth, hypopharynx, and stomach to gain direct access to the lower respiratory tract. ${ }^{395}$ Moreover, the cuff of the endotracheal tube allows pooling of oropharyngeal secretions in the subglottic region, forming an ideal medium for microbial growth, which periodically leaks around the cuff into the trachea. It would probably be more accurate pathogenically to rename VAP as "endotracheal intubation-related pneumonia." This combination of impaired host defenses and continuous exposure of the lower respiratory tract to large numbers of potential pathogens through the endotracheal tube puts the mechanically ventilated patient at great jeopardy of developing VAP.

In order for microorganisms to cause VAP, they must first gain access to the normally sterile lower respiratory 


\begin{tabular}{|c|c|c|c|c|c|c|c|c|}
\hline \multirow[b]{2}{*}{ Type of ICU } & \multirow[b]{2}{*}{ No. of Units } & \multirow[b]{2}{*}{ Ventilator-days } & \multirow[b]{2}{*}{ Pooled Mean } & \multicolumn{5}{|c|}{ Percentile } \\
\hline & & & & $10 \%$ & $25 \%$ & $50 \%$ (Median) & $75 \%$ & $90 \%$ \\
\hline Coronary & 59 & 76,145 & 4.4 & 0 & 1.9 & 4 & 6.8 & 9.8 \\
\hline Cardiothoracic & 47 & 98,358 & 7.2 & 1.2 & 2.9 & 6.3 & 12.6 & 15.5 \\
\hline Medical & 92 & 268,518 & 4.9 & 0.5 & 2.1 & 3.7 & 6.2 & 8.9 \\
\hline Major teaching & 99 & 320,916 & 5.4 & 1.2 & 2.6 & 4.6 & 7.2 & 9.9 \\
\hline All others & 109 & 351,705 & 5.1 & 1.7 & 2.9 & 5.1 & 6.7 & 8.9 \\
\hline Neurosurgical & 29 & 45,073 & 11.2 & 0 & 2.4 & 6.2 & 13.5 & 16.8 \\
\hline Pediatric & 52 & 133,995 & 2.9 & 0 & 0.9 & 2.3 & 4.8 & 8.1 \\
\hline Surgical & 98 & 253,900 & 9.3 & 2.2 & 4.7 & 8.3 & 12.2 & 17.9 \\
\hline Trauma & 22 & 63,137 & 15.2 & 4.3 & 8 & 11.4 & 16.6 & 25.3 \\
\hline Burn & 14 & 23,117 & 12 & - & 一 & - & - & 一 \\
\hline Respiratory & 6 & 18,838 & 4.9 & 一 & 一 & - & - & - \\
\hline \multicolumn{9}{|c|}{$\begin{array}{l}\text { Number of ventilator-days } \\
\text { ICU, intensive care unit. } \\
\text { From National Nosocomial Infections Surveillance System Report, Data Summary from January } 1992 \text { through June 2004. Available at: www.cdc.gov/ } \\
\text { ncidod/dhqp/pdf/nnis/2004NNISreport.pdf (Accessed May 10, 2007) }\end{array}$} \\
\hline
\end{tabular}

tract, where they can adhere to the mucosa and produce sustained infection. Microorganisms gain access by one of four mechanisms (Fig. 51-10): (1) aspiration of microbeladen secretions, either from the oropharynx directly or, secondarily, by reflux from the stomach into the oropharynx, then into the lower respiratory tract ${ }^{396-398}$; (2) inhalation of contaminated air or medical aerosols ${ }^{399}$; (3) direct extension of a contiguous infection such as a pleural space infection ${ }^{400}$; or (4) hematogenous carriage of microorganisms to the lung from remote sites of local infection such as an IVD-related BSI. ${ }^{401}$

Although numerous epidemics of VAP have been caused by contaminated aerosols or medical respiratory devices, ${ }^{94,95,100}$ the preponderance of evidence suggests that most endemic VAPs derive from aspiration of oropharyngeal organisms ${ }^{395,402}$ :

- The oropharynx of critically ill patients is rapidly colonized with the pathogens that cause VAP, especially aerobic gram-negative and $S$. aureus. ${ }^{393}$

- Studies in which multiple anatomic sites are cultured simultaneously over time have shown that the pathogenic microorganisms implicated in VAP are usually first recovered from the oropharynx and later from the tracheobronchial tree and stomach. ${ }^{396-398,403}$ Moreover, heavy oropharyngeal colonization is a powerful independent predictor of subsequent tracheobronchial colonization and VAP. ${ }^{398}$

- Reducing oropharyngeal colonization with topical antimicrobials and antiseptics has been shown to significantly reduce the risk of VAP. ${ }^{404-407}$

By this route, aspiration of oropharyngeal contents containing a large microbial inoculum overwhelms host defenses already compromised by critical illness and the presence of an endotracheal tube, readily leading to the development of VAP.

\section{Microbiology}

Pathogens causing VAP may be part of the host's endogenous flora at the time of hospitalization or may be acquired exogenously after admission to the health care institution, from the hands, apparel or equipment of HCWs, hospital environment, and use of invasive devices (see Fig. 51-10). The normal flora of the oropharynx in the nonintubated patient without critical illness is composed predominantly of viridans streptococci, Haemophilus species, and anaerobes. Salivary flow and proteins (immunoglobulin, fibronectin) are the major host factors maintaining the normal flora of the mouth (and dental plaque). Aerobic gramnegative bacilli are rarely recovered from the oral secretions of healthy patients. ${ }^{408}$ During critical illness, especially in ICU patients, the oral flora shifts dramatically to a predominance of aerobic gram-negative bacilli and $S$. aureus. ${ }^{393}$ Bacterial adherence to the orotracheal mucosa of the mechanically ventilated patient is assisted by reduced mucosal IgA and increased protease production, exposed and denuded mucous membranes, elevated airway $\mathrm{pH}$, increased numbers of airway receptors for bacteria because of acute illness, and antimicrobial use.

Early-onset VAP, which manifests within the first 4 days of hospitalization, is most often caused by communityacquired pathogens, such as S. pneumoniae and Haemophilus species (Fig. 51-11). ${ }^{409}$ However, the microbial spectrum of VAP shifts to typical nosocomial pathogens with increasing lengths of mechanical ventilation and exposure to broad-spectrum antimicrobials (see Fig. 51-11). ${ }^{409}$ That the preponderance of episodes of VAP have a late onset is supported by the fact that the most 


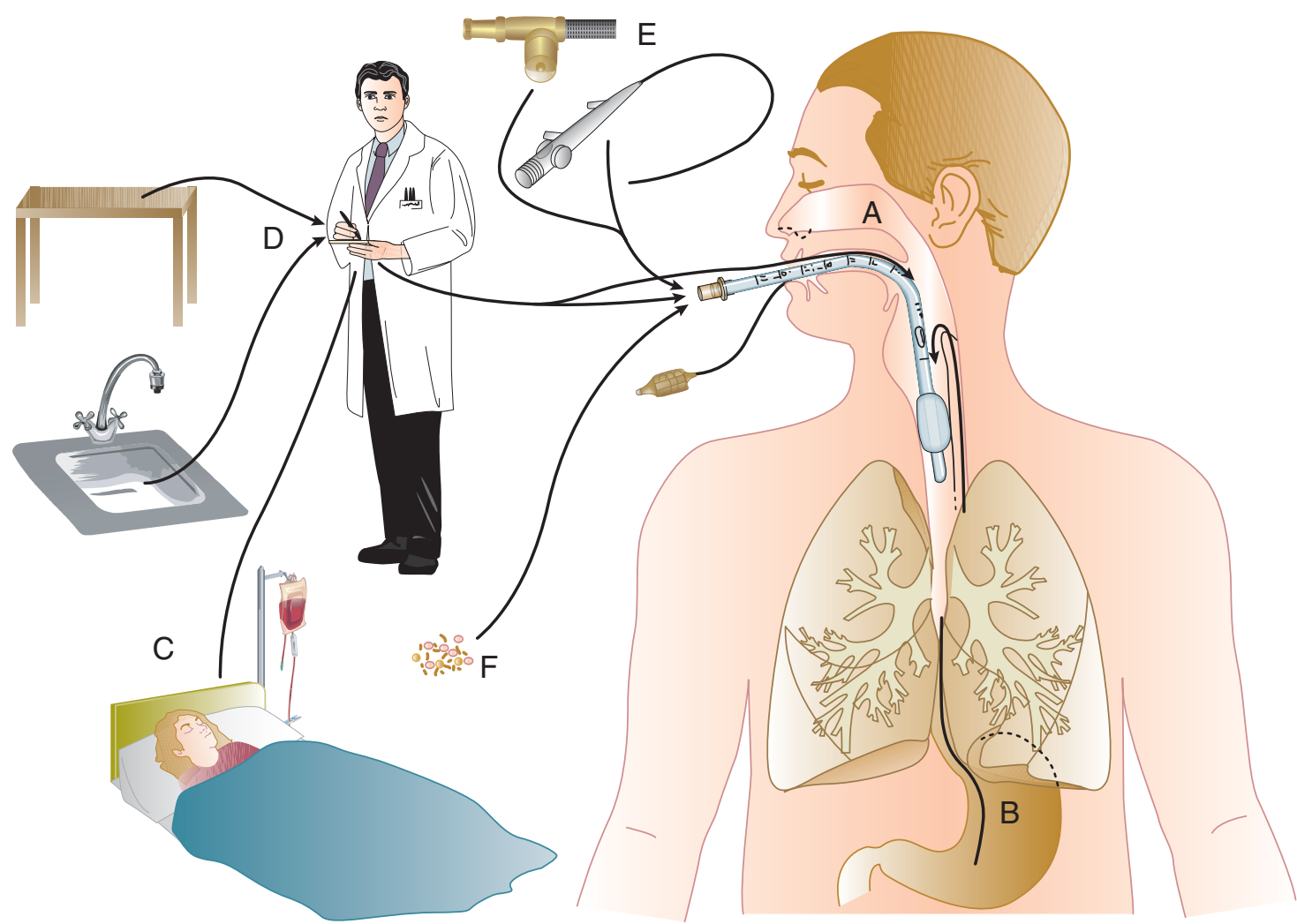

Figure 51-10. Routes of colonization/infection in mechanically ventilated patients. Colonization of the aerodigestive tract may occur endogenously (A and $\mathbf{B}$ ) or exogenously (C through $\mathbf{F})$. Exogenous colonization may result in primary colonization of the oropharynx or may be the result of direct inoculation into the lower respiratory tract during manipulations of respiratory equipment (D), during use of respiratory devices (E), or from contaminated aerosols (F). (From Crnich CJ, Safdar NS, Maki DG: The role of the intensive care environment in the pathogenesis and prevention of ventilator-associated pneumonia. Respir Care 2005;50:813-836.)

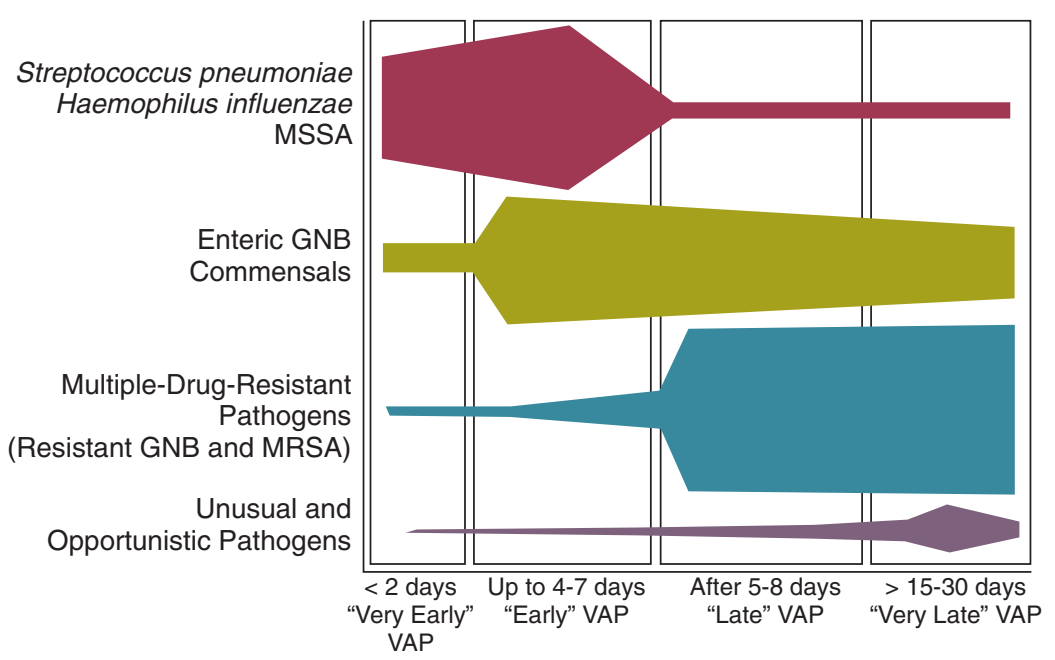

Figure 51-11. Microbial causes of ventilator-associated pneumonia based on increasing length of mechanical ventilation. The relative importance of each microbial category is indicated by the thickness of the bars as they progress through each stage from left to right. GNB, gramnegative bacilli; MRSA, methicillin-resistant S. aureus; MSSA, methicillin-susceptible $S$. aureus; VAP, ventilator-associated pneumonia. (From Park DR: The microbiology of ventilator-assisted pneumonia. Respir Care 2005;50:742-765.)

Periods of Risk by Duration of Mechanical Ventilation

common pathogens recovered from mechanically ventilated patients with pneumonia are $P$. aeruginosa, $S$. aureus, and the Enterobacteriaceae (Fig. 51-12). ${ }^{409,410}$ VAP is polymicrobial in up to $20 \%$ to $40 \%$ of cases. The role of anaerobic bacteria in VAP is not well defined.

\section{Diagnosis}

Hospitals participating in the CDC's National Nosocomial Infection Surveillance system (NNIS) use a standardized definition for $\operatorname{HAP}^{16}$ (see Box 51-1) on the basis of three clinical criteria developed empirically more than 3 decades ago $^{411}$ : (1) systemic signs of infection-fever, tachycardia, and leukocytosis; (2) a new or worsening infiltrate on chest radiograph; and (3) bacteriologic evidence of infection from positive qualitative cultures of endotracheal aspirates. Unfortunately, even when used in combination, the specificity of clinical criteria is poor, with an overall diagnostic accuracy of approximately $60 \%$ in published studies. ${ }^{412,413}$ 


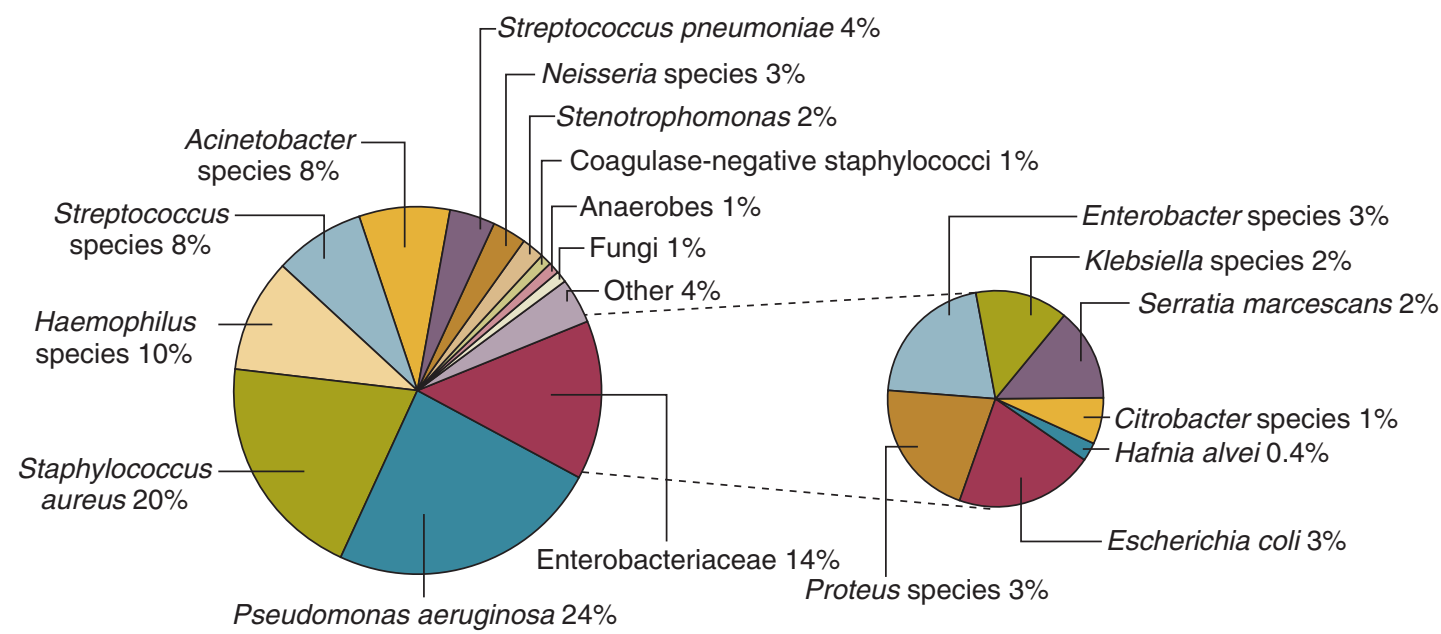

Figure 51-12. Microbial etiology of ventilator-associated pneumonia. The relative proportions of microbial causes of ventilatorassociated pneumonia from 1689 bronchoscopically confirmed cases involving 2490 individual isolates reported in 24 published studies. (From Park DR: The microbiology of ventilator-assisted pneumonia. Respir Care 2005;50:742-765.)

For this reason, most experts have advocated routine use of invasive procedures when VAP is suspected-bronchoalveolar lavage (BAL), cultures of protected specimen brush (PSB) samples obtained by bronchoscopy, or blind (mini)-BAL, on the grounds that these diagnostic techniques have comparable sensitivity, greater specificity, and superior accuracy than clinical criteria alone. ${ }^{410,414-417}$ Whether more rigorous clinical criteria such as the clinical pneumonia infection score (CPIS) ${ }^{418}$ for example, or the use of quantitative cultures of endotracheal aspirates improve diagnostic accuracy without the need for invasive procedures is an unsettled issue. ${ }^{419}$

Although invasive procedures-BAL, PSB, and mini$\mathrm{BAL}$ - are clearly more specific than clinical criteria, their impact on patient outcomes is much less clear ${ }^{420,421}$ Fagon and colleagues ${ }^{420}$ found that patients with suspected VAP who were managed using an invasive diagnostic approachbronchoscopic-guided PSB or BAL-had a significantly reduced 14-day mortality, reduced antibiotic-days, and reduced 28-day mortality on multivariate analysis, compared with patients managed using a clinical diagnostic approach $(\mathrm{HR}=0.65,95 \% \mathrm{CI}=0.46$ to $0.91, P=.01) .{ }^{420}$ Recently, however, Heyland and colleagues ${ }^{421}$ found in a large multicenter Canadian trial that 28-day mortality and targeted antimicrobial use was identical among patients randomized to an invasive versus a clinical diagnostic approach. This study has been criticized for its exclusion of subjects at high risk for infection with antimicrobial-resistant pathogens. ${ }^{422}$ In the absence of definitive data demonstrating the superiority of either approach, the recent American Thoracic SocietySociety of Critical Care Medicine-Infectious Disease Society of America joint guideline acknowledges that both diagnostic approaches are useful and acceptable when evaluating patients with suspected VAP. This puts great weight on an initial Gram stain of a deep tracheal aspirate, however, if no microorganisms are seen, it can be concluded that it is unlikely the patient has bacterial VAP. ${ }^{383}$

\section{Risk Factors}

A number of independent risk factors have been shown to increase the likelihood of developing VAP (Table 51-15). ${ }^{131,410}$ In general, these risk factors can be categorized as (1) factors that increase the likelihood or duration of mechanical ventilation, (2) factors that increase colonization of the oropharynx and gastric mucosa, (3) factors that increase the likelihood of aspiration, and (4) host factors that increase susceptibility to infection.

Prolonged mechanical ventilation or reintubation, or both, are the most powerful predictors of developing VAP. Cunnion and colleagues ${ }^{423}$ found that mechanical ventilation in excess of 24 hours was associated with a 12fold increased risk of developing VAP, and Trouillet found that ventilation longer than 7 days was associated with a sixfold increased risk. ${ }^{424}$ Emergent reintubation also carries a high risk of aspiration and was associated with a sixfold increased risk of VAP in a retrospective study. ${ }^{425}$

Poor dental hygiene increases the bacterial burden in the oropharynx and is an independent risk factor for nosocomial pneumonia. ${ }^{426}$ Likewise, a high gastric $\mathrm{pH}$ $(>5)$ is associated with greatly increased bacterial colonization of the gastric contents, ${ }^{427}$ as well as an increased risk of VAP. ${ }^{428} \mathrm{~A}$ number of studies have found that exposure to antacids or $\mathrm{H}_{2}$-blockers is associated with an increased risk of VAP, ${ }^{53}$ although this has not been a universal finding. ${ }^{429}$

Depressed levels of consciousness, nasogastric tubes, and endotracheal tubes are ubiquitous in the ICU and all increase a patient's risk of aspiration. That an altered level of cognition is associated with an increased risk of aspiration is supported by surveillance data showing increased rates of VAP in trauma and neurosurgical ICUs. ${ }^{133}$ Joshi 
Table 51-15. Independent Risk Factors for Ventilator-Associated Pneumonia in Multivariate Analysis of Published Studies

\section{Host Factors}

Serum albumin, $<2.2 \mathrm{~g} / \mathrm{dL}$

Age, $\geq 60 \mathrm{yr}$

Adult respiratory distress syndrome (ARDS)

Chronic obstructive pulmonary disease or other chronic pulmonary diseases

Coma or impaired consciousness

Burns, trauma

Organ failure

Advanced severity of illness

Large-volume gastric aspiration

Gastric colonization and gastric $\mathrm{pH}$

Upper respiratory tract colonization

Sinusitis

\section{Intervention Factors}

$\mathrm{H}_{2}$ blockers \pm antacids

Paralytic agents, continuous intravenous sedation Receipt of $>4$ units of blood

Intracranial pressure monitoring

Mechanical ventilation in excess of $48 \mathrm{hr}$

Positive end-expiratory pressure

Frequent ventilator circuit changes

Reintubation

Nasogastric tube

Supine head position

Transport out of the intensive care unit

Prior antibiotic therapy

Modified from Chastre J, Fagon JY: Ventilator-associated pneumonia. Am J Resp Crit Care Med 2002;165:867-903.

and colleagues ${ }^{430}$ found that the use of a nasogastric tube was an independent predictor of VAP in a multivariate analysis (OR 6.5, 95\% CI 2.1 to 19.8). Finally, as noted, endotracheal tubes allow pooling of hypopharyngeal secretions that can leak around the cuff directly into the trachea, and a supine position appears to increase the risk of aspiration around the cuff. ${ }^{431}$

Host factors also contribute to an increased risk of developing VAP (see Table 51-15). Conditions such as advanced age, increased severity of illness, and the postsurgical state are rarely modifiable. However, poor nutritional status ${ }^{397}$ oversedation, ${ }^{432}$ transfusion therapy, ${ }^{433}$ and exposure to broad-spectrum antimicrobials ${ }^{424}$ are associated with an increased risk of VAP and are under the control of the clinician.

\section{Prevention}

With an understanding of pathogenesis and epidemiology in hand, clinicians caring for mechanically ventilated patients can implement preventive strategies that can materially reduce the risk of VAP (Table 51-16). Both the CDC HICPAC and Canadian Critical Care Trials Group offer evidence-based guidelines for the prevention of VAP. ${ }^{131,434}$ Their recommendations are very similar, with minor differences. The Canadian guideline focuses exclusively on specific interventions for the prevention of VAP, ${ }^{434}$ whereas the HICPAC guideline incorporates additional guidance for the prevention of nosocomial influenza, legionellosis, and invasive filamentous fungal infections in the hospital. ${ }^{131}$ Recommendations from both guidelines can be divided into general, nonpharmacologic, and pharmacologic preventive measures (see Table 51-16). ${ }^{435}$ The general measures employed to reduce VAP including education, infection control, hand hygiene, and reliable disinfection and sterilization of respiratory care equipment are discussed elsewhere in this chapter.

\section{Nonpharmacologic Preventive Measures}

Avoiding prolonged intubation and reintubation-if avoiding intubation altogether is not feasible-offers the greatest promise for reducing an individual patient's risk of developing VAP. ${ }^{425}$ The use of noninvasive ventilation in order to avoid endotracheal intubation has been shown to be successful in reducing rates of nosocomial pneumonia in a number of studies ${ }^{436,437}$ and may abrogate the need for reintubation in selected patients who prematurely extubate themselves. ${ }^{131}$ The implementation of weaning protocols has also been shown to significantly reduce the duration of mechanical ventilation, ${ }^{438,439}$ health care costs, ${ }^{438,439}$ and institutional rates of VAP. ${ }^{440,441}$ Early tracheostomy - within 1 week of intubation - has been advocated as a method for reducing the risk of VAP in patients likely to require prolonged mechanical ventilation. However, randomized trials, admittedly of limited power, have not found significant benefit with this approach $^{442}$ and early tracheostomy is not currently recommended by most authorities. ${ }^{131,434}$

As noted earlier, supine positioning of the mechanically ventilated patient's head has been shown to increase the risk of gastro-esophageal-pharyngeal aspiration. ${ }^{431} \mathrm{~A}$ simple solution to this threat is to elevate the head of the patient's bed 35 to 45 degrees. Drakulovic and colleagues ${ }^{443}$ found that patients whose torso and head were kept elevated at 45 degrees had much lower rates of microbiologically confirmed pneumonia compared with patients cared for in a 0 -degree supine position $(5 \%$ versus $23 \%, P=.018) .{ }^{443}$ In reality, maintaining elevation of the head in excess of 45 degrees on a consistent basis is actually quite difficult and uncommonly achieved in practice. A recent randomized study that sought to maintain head elevation above 45 degrees for $85 \%$ of the study period found that head elevation in the intervention arm only averaged 28.1 degrees. ${ }^{444}$ Perhaps as a result of failure to successfully achieve adequate elevation, no reductions in the rate of VAP were seen.

Although data on the effect that comprehensive oral care has on risk of infection are limited ${ }^{445}$ maintaining adequate dental hygiene is considered an important component of VAP prevention. ${ }^{131}$ Binkley and colleagues ${ }^{446}$ found that although a majority of nurses caring for patients undergoing mechanical ventilation appreciated the 
- Educate all health care workers involved with the care of mechanically ventilated patients on the risks and methods of preventing ventilator-associated pneumonia

- Perform adequate hand hygiene between patient contacts

- Use gloves for handling respiratory secretions or objects contaminated with respiratory secretions

- Conduct surveillance for bacterial pneumonia in ICU patients using NNIS definitions. Include data on causative organisms and their antimicrobial susceptibility patterns. Express data as rates to assist intrahospital comparisons

- Do not routinely perform cultures of patients, equipment, or environment in the absence of an outbreak

- Thoroughly clean all devices to be sterilized and disinfected

- Use steam sterilization or wet heat pasteurization for reprocessing of heat-stable semicritical devices and low-temperature sterilization for heator moisture-sensitive devices

Use sterile water for rinsing reusable semicritical devices

- Change ventilator circuit only when they become soiled

- Periodically drain and discard condensate from ventilator circuits

- Clean, disinfect, rinse with sterile water, and dry in-line nebulizers between treatments on the same patient

- When possible, use aerosolized medications in single-use vials

IA IA -

IB

IB

IA

IA

$-$

$-$

Recommended

-

$-$

-

Nonpharmacologic Measures to Reduce Pneumonia

- Oral (non-nasal) intubation

- Remove nasogastric and endotracheal tubes as soon as clinically feasible

- Avoid unnecessary reintubation

- When feasible, use noninvasive ventilation to avoid the need for intubation or reintubation

- Early tracheostomy

Semirecumbent positioning of the patient

- Implement a comprehensive oral-hygiene program for mechanically ventilated patients

- If feasible, use an endotracheal catheter that allows for continuous or frequent subglottic suctioning

- Humidification with heat and moisture exchanger (HME)

- Closed multiuse catheters for airway secretion suctioning

Kinetic bed therapy

Pharmacologic Measure to Reduce Pneumonia

- Immunize all patients at risk for pneumococcal infection

- Immunize all patients at risk for influenza

- Routine use of chlorhexidine oral rinse

- Targeted use of chlorhexidine oral rinse in postcardiac surgery patients

- Oral decontamination with topical antimicrobial agents

- Preferential use of sucralfate for stress bleeding prophylaxis

- Selective digestive decontamination

- Acidification of gastric feedings

- Systemic antimicrobials to prevent development of pneumonia

- Cycling of antibiotic classes to reduce resistance in the ICU

*Taken from CDC/HICPAC system of weighting recommendations based on scientific evidence. IA, strongly recommended for implementation and supported by well-designed experimental, clinical, or epidemiological studies. IB, strongly recommended for implementation and supported by some experimental, clinical, or epidemiological studies and a strong theoretical rationale. IC, required by state or federal regulations, rules or standards. II, suggested for implementation and supported by suggestive clinical or epidemiological trials or a theoretical rationale. Unresolved issue, an unresolved issue for which evidence is insufficient or no consensus regarding efficacy exists. NR, no recommendation for or against at this time.

${ }^{\dagger}$ Recommended in patients without hemoptysis or high minute ventilation. Exchanger should be replaced weekly.

FTopical or systemic antimicrobial agents alone are not recommended. Insufficient evidence on antibiotic resistance and cost-effectiveness exists to recommend combination topical and systemic therapy.

CCCTG, Canadian Critical Care Trials Group; HICPAC, Healthcare Infection Control Practices Advisory Committee.

Modified from Tablan OC, Anderson LJ, Besser R, et al: Guidelines for preventing health-care-associated pneumonia, 2003: Recommendations of

CDC and the Healthcare Infection Control Practices Advisory Committee. MMWR Recomm Rep 2004;53(RR-3):1-36 and Dodek P, Keenan S, Cook

$\mathrm{D}$, et al: Evidence-based clinical practice guideline for the prevention of ventilator-associated pneumonia. Ann Intern Med 2004;141:305-313. 
importance of dental hygiene, the methods used to provide this varied considerably. Until more data are available on specific dental hygienic practices, it is recommended that mechanically ventilated patients have their teeth brushed daily, undergo oral cleansing every 2 to 4 hours, undergo routine suctioning to reduce accumulation of fluids in the oropharynx, and have a mouth moisturizer applied to their lips to prevent cracking. ${ }^{447}$ The periodic instillation of a topical oral antiseptic solution is an additional promising intervention ${ }^{447}$ and is discussed under pharmacologic preventive measures later.

The use of a modified endotracheal tube that has a separate ventral drainage tube for continuous or intermittent suctioning of subglottic secretions has been evaluated in a number of studies. ${ }^{448,449}$ Subglottic suctioning reduced the rate of VAP significantly in all but one of these studies. ${ }^{449}$ However, in this latter study, the time to onset of VAP was delayed significantly ( 5.9 days versus 2.9 days, $P=.006),{ }^{449}$ and recent evidence-based guidelines have recommended the use of endotracheal tubes that allow for suctioning of subglottic secretions. ${ }^{131,434}$ Nevertheless, the use of an endotracheal tube that allows for subglottic suctioning did not reduce the duration of mechanical ventilation or ICU mortality in the studies done, which, coupled with the increased cost of the tube and propensity of the suction lumen to occlude, has limited wider adoption of this technology in practice. ${ }^{450}$

The evidence that heat and moisture exchangers (HMEs) are associated with a reduced risk of VAP is mixed. Only one of six published trials found a statistically significant reduction in VAP with use of HMEs (RR 0.41, 95\% CI 0.20 to $0.86, P=.02) .{ }^{451}$ However, pooling data from a recent systematic review ${ }^{452}$ and a subsequently published randomized trial ${ }^{453}$ shows that HMEs reduce the risk of VAP by $38 \%$ (RR $0.62,95 \%$ CI 0.43 to $0.89, P=.012$ ). The use of HMEs has been recommended by authors of a systematic review ${ }^{454}$ and is currently recommended by the Canadian Critical Care Trials Group. ${ }^{434}$ However, HICPAC made no recommendation for the use of HMEs because five of six published trials failed to demonstrate a statistically significant reduction in the rate of VAP. ${ }^{131}$ Heat exchange moisturizers become readily occluded in patients with airway hemorrhage and can increase airway resistance. As a result, they should not be used in patients with hemoptysis or those requiring a high-minute ventilation. ${ }^{434}$ Finally, the membranes of HMEs can become colonized with bacteria and should be replaced weekly, according to current guidelines. ${ }^{434}$

The availability of in-line multiuse suction catheters abrogates the need to open and manipulate the endotracheal circuit, theoretically reducing the risk of exogenous contamination. ${ }^{455}$ Despite their theoretical benefit, prospective studies have not consistently showed that in-line suction catheters are associated with a reduced risk of VAP. ${ }^{456-458}$ Although in-line suction catheters do not appear to increase the risk of VAP, they are more time efficient for nursing personnel and respiratory therapists, and are more cost effective than open suction catheters. ${ }^{434}$ Kollef and colleagues ${ }^{459}$ found that rates of VAP were identical in patients randomized to as-needed changes of their inline suction catheter versus those who had their catheter changed every 24 hours (14.7\% versus $14.8 \%)$. As a result, there is no compelling evidence that in-line suction catheters should be periodically changed, unless clinically indicated.

\section{Pharmacologic Preventive Measures}

Antacids and $\mathrm{H}_{2}$-blockers have been used extensively in the ICU setting to prevent stress ulcer bleeding but have been associated with an increased risk of developing of VAP because they lead to bacterial overgrowth of the gastric contents. ${ }^{53}$ Sucralfate prevents stress ulcer bleeding without reducing gastric $\mathrm{pH}$ but is more difficult to administer and is less effective than acid-reducing agents. ${ }^{429}$ The results of clinical trials examining these two competing strategies for preventing gastrointestinal hemorrhage in the ICU have been mixed, with earlier trials favoring the use of sucralfate. ${ }^{53}$ However, more recently published trials suggest only a small incremental increased risk of VAP with $\mathrm{H}_{2}$-blockers ${ }^{429,460,461}$ and most experts feel that this risk is more than offset by their superior capacity to prevent stress ulcer bleeding. ${ }^{131,434}$

Selective digestive decontamination (SDD) is one of the most extensively studied preventive interventions in critical care medicine, yet the role for SDD continues to generate vigorous debate as to its overall benefit. ${ }^{462,463}$ A more detailed discussion on the risks and benefits of this intervention is provided later in this chapter. Most U.S. experts believe that SDD has the potential to increase infection caused by multiresistant bacteria, particularly in settings with high rates of endemic antimicrobial resistance. ${ }^{464,465}$ Until well-designed multicenter trials are done, proving that SDD does not adversely effect the ICU ecology, it is likely that North American guidelines will continue to discourage its use. $^{131,434}$

The isolated use of parenteral antimicrobials for prevention of VAP has not met with much success, ${ }^{464}$ but selective antimicrobial decontamination of the oropharynx, without the use of enteral or systemic agents, reduced the risk of VAP nearly $70 \%(\mathrm{RR}=0.33,95 \%$ CI 0.16 to $0.67, P=.001)$ in a recent trial. ${ }^{405}$ This study reemphasized the primary role of oropharyngeal colonization in the pathogenesis of VAP but engenders the same concerns as SDD over its potential for promoting antimicrobial resistance. However, it has facilitated the idea that topical decolonization of the oropharynx with nonantimicrobial agents might be able to materially reduce the risk of VAP without the potential for emergence of antimicrobial resistance. A recent meta-analysis of seven randomized trials that enrolled 914 mechanically ventilated patients found that topical chlorhexidine applied to the oropharynx reduced the risk of VAP by nearly $30 \%$ (RR $=0.74,95 \%$ CI 0.56 to $0.96, P=.02$ ), although there was no significant impact on mortality. ${ }^{407}$ The beneficial effects of chlorhexidine appear to be most pronounced in post-cardiac surgery patients, ${ }^{466,467}$ prompting HICPAC to recommend its use in this subpopulation. ${ }^{131}$ 


\section{Catheter-Associated Urinary Tract Infection}

\section{Incidence and Impact}

Each year, urinary catheters are inserted in more than 5 million patients in acute-care hospitals and extended-care facilities. ${ }^{468}$ Catheter-associated urinary tract infection (CAUTI) is the most common nosocomial infection in hospitals and nursing homes, comprising more than $40 \%$ of all institutionally acquired infections. ${ }^{133}$

Nosocomial bacteriuria or candiduria develops in up to $25 \%$ of patients requiring a urinary catheter for more than 7 days, with a daily risk of $5 \% .{ }^{468}$ CAUTI is the second most common cause of nosocomial bloodstream infection ${ }^{469}$; some studies have also found increased mortality associated with CAUTI. ${ }^{470}$ Although most CAUTIs are asymptomatic, ${ }^{471}$ rarely extend hospitalization, and add only $\$ 500$ to $\$ 1000$ to the direct costs of acute-care hospitalization, ${ }^{472}$ asymptomatic infections commonly precipitate unnecessary antimicrobial-drug therapy. ${ }^{473}$ CAUTIs comprise perhaps the largest institutional reservoir of nosocomial antibiotic-resistant pathogens, the most important of which are multidrug-resistant Enterobacteriaceae other than Escherichia coli such as Klebsiella, Enterobacter, Proteus, and Citrobacter; Pseudomonas aeruginosa; enterococci and staphylococci; and Candida spp. ${ }^{474}$

\section{Pathogenesis}

Excluding rare hematogenously derived pyelonephritis, caused almost exclusively by $S$. aureus, most microorganisms causing endemic CAUTI derive from the patient's own colonic and perineal flora or from the hands of health care personnel and gain access to the patient's urinary tract during catheter insertion or manipulation of the collection system. ${ }^{260}$ Organisms gain access in one of two ways. Extraluminal contamination may occur early, by direct inoculation when the catheter is inserted, or later, by organisms ascending from the perineum by capillary action in the thin mucous film between the external catheter surface and the urethral wall. Intraluminal contamination occurs by reflux of microorganisms gaining access to the catheter lumen from failure of closed drainage or contamination of urine in the collection bag. Recent studies suggest that CAUTIs most frequently stem from microorganisms gaining access to the bladder extraluminally, ${ }^{475}$ but both routes are important.

Most infected urinary catheters are covered by a thick biofilm containing the infecting microorganisms embedded in a matrix of host proteins and microbial exoglycocalyx. ${ }^{476} \mathrm{~A}$ biofilm forms on the intraluminal or extraluminal surface of the implanted catheter, or both, usually advancing in a retrograde fashion. The role of the biofilm in the pathogenesis of CAUTI has not been established. However, anti-infective-impregnated and silver-hydrogel catheters, which inhibit adherence of microorganisms to the catheter surface, significantly reduce the risk of CAUTI, ${ }^{477}$ particularly infections caused by gram-positive organisms or yeasts, which are most likely to be acquired extralumi- nally from the periurethral flora. These data suggest that microbial adherence to the catheter surface is important in the pathogenesis of many, but not all, CAUTIs. Infections in which the biofilm does not play a pathogenic role are probably caused by mass transport of intraluminal contaminants into the bladder by retrograde reflux of microbe-laden urine when a catheter or collection system is moved or manipulated.

\section{Prevention}

Several catheter-care practices are universally recommended to prevent or at least delay the onset of CAUTI ${ }^{260}$ : most importantly, avoiding unnecessary catheterizations; considering using a condom catheter in a male or a suprapubic catheter; having trained professionals insert catheters aseptically; removing the catheter as soon as no longer needed; maintaining uncompromising closed drainage; ensuring dependent drainage as much as possible; minimizing manipulations of the system; and separating catheterized patients geographically on the patient care unit.

As noted earlier, technologic innovations to prevent nosocomial infection are most likely to be effective if they are based on a clear understanding of the pathogenesis and epidemiology of the infection. Novel technologies must be designed to block CAUTI by either the extraluminal or intraluminal routes, or both. Medicated catheters, which reduce adherence of microorganisms to the catheter surface, may confer the greatest benefit for preventing CAUTI. Two catheters impregnated with antiinfective solutions have been studied in randomized trials, one impregnated with the urinary antiseptic nitrofurazone $^{478}$ and the other with a new broad-spectrum antimicrobial-drug combination, minocycline and rifampin. ${ }^{479}$ Both catheters showed a modest reduction in bacterial CAUTIs; however, the studies were small, and the risk of selection of antimicrobial drug-resistant uropathogens was not satisfactorily resolved. Silver compounds have also been studied for coating urinary catheters. A meta-analysis of eight randomized trials comparing silver oxide or silver alloy catheters with standard nonimpregnated catheters found that silver alloy, but not silver oxide, catheters were associated with a reduced risk of CAUTI. ${ }^{480}$ Recommendations for the prevention of CAUTI are summarized in Table 51-17.

\section{Control of Antibiotic Resistance}

During the past 55 years, more than 14 different classes of parenteral antimicrobials and several hundred antimicrobial compounds have been introduced into clinical use. In the 1960s, public health officials confidently declared that the war against infectious diseases was almost over. Unfortunately, it is not clear which side will be victorious. Although the greatest strides in our struggles with infectious diseases have resulted from improvements in hygiene and social conditions, the growing losses of our antibiotic armamentarium as a result of surging bacterial resistance could ultimately be disastrous for ICU patients if the tide is not stemmed. 
Table 51-17. Recommendations for Prevention of Catheter-Associated Urinary Tract Infection

\begin{tabular}{|c|c|}
\hline Recommendation & $\begin{array}{l}\text { Strength of } \\
\text { Recommendation* }\end{array}$ \\
\hline $\begin{array}{l}\text { Educate personnel in correct } \\
\text { techniques of catheter insertion } \\
\text { and care. }\end{array}$ & 1 \\
\hline - Catheterize only when necessary. & 1 \\
\hline Emphasize hand hygiene. & I \\
\hline $\begin{array}{l}\text { - Insert catheter using aseptic } \\
\text { technique and sterile equipment. }\end{array}$ & 1 \\
\hline Secure catheter properly. & 1 \\
\hline . Maintain closed sterile drainage. & I \\
\hline Obtain urine samples aseptically. & 1 \\
\hline Maintain unobstructed urine flow. & I \\
\hline $\begin{array}{l}\text { Periodically re-educate personnel in } \\
\text { catheter care. }\end{array}$ & II \\
\hline - Use smallest suitable bore catheter. & II \\
\hline $\begin{array}{l}\text { Avoid irrigation unless needed to } \\
\text { prevent or relieve obstruction. }\end{array}$ & II \\
\hline $\begin{array}{l}\text { Refrain from daily meatal care with } \\
\text { either of the regimens discussed } \\
\text { in text. }\end{array}$ & II \\
\hline $\begin{array}{l}\text { Do not change catheters at } \\
\text { arbitrary fixed intervals. }\end{array}$ & II \\
\hline $\begin{array}{l}\text { Consider alternative techniques of } \\
\text { urinary drainage before using an } \\
\text { indwelling urethral catheter. }\end{array}$ & III \\
\hline
\end{tabular}

- Replace the collecting system when III sterile closed drainage has been violated.

Spatially separate infected and III uninfected patients with indwelling catheters.

- Avoid routine bacteriologic monitoring.

- Consider the use of a nitrofurantoin or silver hydrogel catheter.

*Novel technology was not addressed in this guideline. Category I, strongly recommended for adoption; Category II, moderately recommended for adoption; Category III, weakly recommended for adoption.

Modified from Wong ES: Guideline for prevention of catheterassociated urinary tract infections. Am | Infect Control 1983;11:28-36.

\section{Evolution of Antibiotic Resistance in Intensive Care Units}

Antimicrobial resistance has evolved through several phases. In the 1970s and 1980s, resistance of aerobic gram-negative bacilli was the major concern, and P. aeruginosa, with its broad range of intrinsic and acquired resistances, was the quintessential nosocomial pathogen. By the 1990s, the availability of antibiotics from a variety of distinct classes-aminoglycosides, broad-spectrum penicillins (e.g., piperacillin), monobactams (e.g., aztreonam), carbapenems (e.g., imipenem), $\beta$-lactam-beta- lactamase inhibitors (e.g., piperacillin-tazobactam), trimethoprim-sulfamethoxazole, and fluoroquinolonespromised a respite from concerns about resistance in aerobic gram-negative bacilli. During this period, however, gram-positive cocci gained prominence, and MRSA $\beta$-lactam-resistant coagulase-negative staphylococci and VRE became the major problem nosocomial pathogens. Antibiotic pressure, deriving first from the widespread use of third-generation cephalosporin antibiotics in hospitals, is often cited as a major factor in the emergence of MRSA. Co-emerging as nosocomial pathogens with MRSA have been methicillin-resistant coagulase-negative staphylococci, which have become the leading cause of IVD-related BSI and prosthesis-related surgical site infections.

In the early 1990s VRE burst onto the hospital and ICU scene in the United States and within a few years became entrenched in most tertiary medical centers. Heavy use of vancomycin, often as empiric treatment in response to concerns about MRSA, was probably the initial factor driving the emergence of VRE. In most settings, however, exposure to cephalosporins and antimicrobials with antianaerobic activity have emerged as the greatest risk factors for nosocomial colonization or infection by VRE. The mid-1990s witnessed growing problems with resistance in fungi and shifts to non-Candida albicans species, representing the effects of heavy empirical use of azoles such as fluconazole in hospitals during this period.

The ICU component of the CDC's NNIS system powerfully reaffirms the rapidly rising rates of bacterial resistance in U.S. ICUs during the past 20 years (see Fig. $51-2){ }^{133}$

\section{Forces Driving Resistance}

To a large extent, emergence of antimicrobial resistance reflects the combined effects of genetic selection, antibiotic pressures, and the frequency of cross-infection in ICUs. For some resistance mechanisms (e.g., extendedspectrum $\beta$-lactamases [ESBLs] that confer resistance to third-generation cephalosporins such as ceftazidime), a shift of single amino acid in existing resistance genes can lead to new, inactivating enzymes. For other resistant bacteria, such as penicillin-resistant pneumococci, multiple resistance genes must be cobbled together in a specific, exacting sequence, which may take years to evolve, emerge, and spread.

Antibiotic pressures provide the necessary Darwinian forces that amplify these genetic changes ${ }^{481}$ Usually, resistance emerges to a specific agent that is used most heavily and, hence, provides the greatest pressure. In some instances, genetic linkage of resistance mechanisms to unrelated classes of antimicrobials results in the capacity of heavy use of one drug class to select for resistance to a different class. For example, use of trimethoprimsulfamethoxazole has been associated statistically with emergence of ceftazidime-resistant $E$. coli and $K$. pneumoniae as a result of linkage on a single plasmid of genes that encode production of ESBLs and trimethoprimsulfamethoxazole resistance. A large proportion of 
extended-spectrum $\beta$-lactamase producing gramnegative bacilli are also resistant to fluoroquinolones. ${ }^{482,483}$

In epidemiologic and clinical studies of antibiotic resistance, there is always a proportion of patients in whom resistance is found without exposure to the problem antibiotic. These patients usually have other important risk factors, such as increased severity of underlying disease, extremes of age, presence of invasive devices, recent surgery, or proximity to patients who are infected or colonized with antibiotic-resistant bacteria. In these cases the presence of antibiotic-resistant strains is most often the consequence of patient-to-patient spread, usually on the contaminated hands of HCWs; occasionally, spread results from a contaminated common source, such as an inadequately cleaned piece of equipment. Studies of HCW hand hygiene show that rates of handwashing between patient contacts range from $25 \%$ to $50 \%$, at best, and are inadequate to control resistance, especially in ICUs, where the staff are extremely busy and less likely to be attentive to hand hygiene. ${ }^{165}$

\section{Controlling Antimicrobial Resistance in the Intensive Care Unit}

Stemming the tide of antimicrobial resistance requires a multifaceted approach (see Box 51-3), especially in ICUs, where antibiotic pressures and lapses in hospital hygiene are usually greatest. First, active surveillance for resistant bacteria is essential to provide an understanding of local problems and needs. To support surveillance and treatment, cultures must be obtained from suspected sites of infection before empiric antibiotic therapy is initiated. The benefit of routine surveillance cultures (e.g., periodic cultures of sputum specimens or rectal swabs) for assessing rates of colonization by resistant bacteria in ICUs will depend on how such cultures are used.

Second, when rates of resistance begin to increase, molecular typing, such as by pulsed-field gel electrophoresis, can differentiate spread of a single strain (clonal expansion) - which suggests person-to-person or common source transmission-from spread of multiple strains (polyclonal expansion), which suggests emergence of resistance in individual patients as a result of antibiotic pressures or exogenous introduction of multiple resistant strains. Often, these problems-clonal and polyclonal-coexist.

Third, the importance of hand hygiene must be stressed at all times. Aggressive hand hygiene campaigns, with adherence monitoring and feedback of ward and even individual results, may achieve compliance rates as high as $70 \%$. For some situations (e.g., when there is a large resistance iceberg and extensive patient colonization by antibiotic resistant bacteria), these levels of adherence may not be sufficient to control cross-infection. Response to this problem has been to encourage "universal gloving," in addition to wider use of alcohol-based hand rubs (a "belt-and-suspender" approach) to bridge the gap left by incomplete attention to hand hygiene even in the best of circumstances. Use of universal gloving has been successful in controlling spread of aminoglycoside-resistant gram-negative bacilli in ICUs and C. difficile-related diar- rhea. ${ }^{224,241}$ Because patients' intact skin and the environment in patient rooms may be a source of resistant bacteria, such as VRE, we recommend that disposable examination gloves be worn for all contact with ICU patients or their environment. Because gloves are not a total barrier, they must be removed and hands disinfected by an alcohol hand rub between patient contacts.

Fourth, antimicrobial stewardship is essential (see Table 51-18). ${ }^{253}$ The primary goal of antimicrobial stewardship is to optimize clinical outcomes while minimizing unintended consequences of antimicrobial use such as toxicity, emergence of resistance, and $C$. difficile-associated diarrhea. Because antimicrobial use drives antimicrobial resistance, the frequency of inappropriate antimicrobial use can be used as a surrogate marker for antimicrobial resistance. Both antimicrobial stewardship and a comprehensive infection control program are essential to limiting the emergence and transmission of antimicrobial-resistant pathogens. Most studies assessing the utility of antimicrobial stewardship have focused on adults in ICUs, where the burden of antimicrobial resistance is greatest.

A comprehensive evidence-based stewardship program to combat antimicrobial resistance is typically a multifaceted, multidisciplinary program; the size and complexity of the management team and the specific measures applied to optimize prescribing vary on the basis of local antimicrobial use patterns, resistance trends, and available resources. The two core strategies that provide the foundation for a successful antimicrobial stewardship program are (1) prospective audits, with intervention and feedback; and (2) formulary restriction and preauthorization. ${ }^{253}$

Several studies have shown that prospective audits of antimicrobial use with intervention and feedback are an effective means of reducing inappropriate antimicrobial use. ${ }^{484,485}$ In a randomized trial conducted at a 600-bed tertiary teaching hospital, inpatients receiving parenteral antimicrobial therapy were randomized to an intervention group that received suggestions for optimal antimicrobial use from an infectious diseases physician or to no interventions. Physicians in the intervention group implemented $85 \%$ of the suggestions they received, which resulted in 1.6 fewer days of parenteral therapy and $\$ 400$ savings per patient. Similar results have been noted in trials undertaken in community hospitals. ${ }^{484}$ If daily review of antimicrobial use is not feasible, review of antimicrobial usage 3 days a week may still have a significant impact. Effective audit with intervention and feedback can be undertaken most easily with automated computer surveillance of antimicrobial use, allowing the targeting of specific units where the problems are greatest.

Formulary restriction and preauthorization requirements for specific agents are now common in most hospitals. Antimicrobial restriction is unequivocally the most effective method of controlling antimicrobial use. ${ }^{486,487}$ However, it is unclear whether antimicrobial restriction achieves the more important outcome, reducing antimicrobial resistance. Several studies of outbreaks of $C$. difficile-associated diarrhea have shown abrupt cessation of the outbreak following restriction (and greatly reduced use) of one or more key antimicrobials such as clindamy- 
Table 51-18. Recommendations for Developing an Institutional Program to Enhance Antimicrobial Stewardship

Recommendation

Level of Evidence

- Create a multidisciplinary antimicrobial stewardship team, including an infectious disease physician

A-II

and a clnical pharmacist with infectious disease training

- Include, if possible, a clinical microbiologist, an information systems specialist, an infection control professional, and hospital epidemiologist

- Foster collaboration between the antimicrobial stewardship team and the hospital infection control committee

- Create a climate of support and collaboration between the antimicrobial stewardship team and the hospital administration and medical staff leadership

- Develop infrastructure to measure antimicrobial use and track use on ongoing basis

is

- Employ a system of prospective audit of antimicrobial use with direct interaction and feedback to the prescriber by an infectious disease physician or a clinical pharmacist with infectious disease training

- Use formulary restrictions and preauthorization requirement to reduce antimicrobial use and cost

Provide education to health care providers regarding stewardship strategies

Education must be combined with active interventions to improve antimicrobial prescribing practices

- Develop evidence-based multidisciplinary guidelines incorporating local microbiology and resistance patterns to improve antimicrobial utilization

- No recommendation can be made regarding antimicrobial cycling as a means of preventing or reducing antimicrobial resistance

- Use antimicrobial order forms as a component of antimicrobial stewardship

$$
\text { A-III }
$$

A-III

No recommendation can be made regarding the routine use of combination therapy to prevent emergence of resistance

- Streamline or de-escalate antimicrobial therapy on the basis of culture results

Optimize antimicrobial dosing on the basis of individual patient characteristics, causative organisms, site of infection, and pharmacokinetic and pharmacodynamic characteristics of the drug

- Use health care information technology such as electronic medical records, computerized physician order entry and clinical decision support to improve antimicrobial prescribing

- Use computer-based surveillance for more efficient targeting of antimicrobial interventions, tracking of resistance patterns, identification of nosocomial infections and adverse drug reactions

- Engage the clinical microbiology laboratory to participate in antimicrobial stewardship by providing patient-specific culture and susceptibility data and by assisting infection control efforts in the surveillance of resistant organisms and in the molecular epidemiologic investigation of outbreaks

Determine the impact of antimicrobial stewardship by measuring process and outcomes

A-III

A-II

A-I

A-

A-II

A-III

B-II

A-I

C-II

B-II

C-II

Based on the Infectious Diseases Society of America grading system for ranking recommendations in clinical guidelines. A, good evidence to support a recommendation for use; B, moderate evidence to support a recommendation for use; C, poor evidence to support a recommendation for use; I, evidence from $>1$ properly randomized, controlled trial; II, evidence from $>1$ well-designed clinical trial, without randomization; from cohort or case-controlled analytic studies; from multiple time-series; III, evidence from expert opinion.

Modified from Dellit TH, Owens RC, McGowan JE Jr, et al: Infectious Diseases Society of America and the Society for Healthcare Epidemiology of America guidelines for developing an institutional program to enhance antimicrobial stewardship. Clin Infect Dis 2007;44:159-177.

cin or third-generation cephalosporins. ${ }^{486}$ However, other studies have documented inexorably rising resistance rates in nosocomial pathogens despite a rigorous program of antimicrobial restriction. ${ }^{488}$ One explanation for this increase in resistance may be the compensatory increase in usage of broad-spectrum antimicrobials other than the restricted agent, thus counteracting any benefit of restriction. Furthermore, restricting use of a single drug to reduce antimicrobial resistance may be ineffective because cross-resistance in bacterial species to more than one class of antimicrobials is the rule in nosocomial organisms.

One or both of the core strategies should be adopted and supplemented by close collaboration among a core antimicrobial stewardship team, infection control personnel, health care providers, and hospital administration.
Beyond the two major mechanisms of antimicrobial stewardship mentioned earlier, other elements that should be incorporated into an institutional antimicrobial stewardship program include education of health care pro-viders; however, passive educational efforts such as conference presentations, teaching sessions, and provision of guidelines are only marginally effective in the absence of other active interventions. ${ }^{489}$ Clinical practice guidelines are being introduced with increasing frequency; however, the impact of these guidelines on provider behavior and clinical outcomes has been difficult to measure. Guidelines tailored to local antimicrobial resistance patterns and antimicrobial use trends may have more impact than a generic clinical pathway. 
Interest has been sparked in ICUs by the reborn concept of antibiotic cycling. ${ }^{40,491}$ The most recent experiences have evaluated switch therapy ${ }^{492}$ for empiric antibiotic use, rather than actual cycling, and have shown beneficial reductions in resistance among gram-negative bacilli ${ }^{493}$ and in the prevalence of VRE. Such approaches, as well as true cycling through different antimicrobial classes, may be effective over limited periods in closed environments such as ICUs, by transiently reducing selection pressure and thus resistance to the restricted agent. Yet studies have thus far not shown a consistent long-term benefit with cycling, and mathematical models do not predict that cycling will be an effective measure to reduce antimicrobial resistance. ${ }^{494}$

Antimicrobial order forms reduce antimicrobial usage through the use of automatic stop orders and the requirement for physician justification. ${ }^{495}$ Streamlining or deescalation of therapy based on culture data is an essential component of appropriate antimicrobial use, with studies showing substantial reductions in days of antimicrobial use and cost savings. ${ }^{496,497}$

Computer order entry provides needed information at the moment in a neutral, nonjudgmental, fact-based format; this system is efficient, well accepted, and holds the promise to change prescribing behaviors materially. ${ }^{498,499}$

Effective antimicrobial stewardship programs can be financially self-supporting and improve patient care. Studies have shown reductions in antimicrobial usage from $22 \%$ to $36 \%$, with annual savings of $\$ 200,000$ to $\$ 900,000$ in larger teaching hospitals and community hospitals. A recent guideline from the Infectious Diseases Society of America and the Society for Healthcare Epidemiology of America provides detailed recommendations for developing institutional programs of antimicrobial stewardship, which are summarized in Table 51-18. ${ }^{253}$

\section{AVANT GARDE CONTROL MEASURES}

\section{Selective Digestive Decontamination}

Intense interest has arisen in Europe and the United States $457-459$ over the use of "selective digestive decontamination" (SDD) for prevention of bacterial pneumonia and other nosocomial infections in mechanically ventilated ICU patients. This novel therapy is based on the premise that the upper respiratory tract flora exists in a continuum with the gastrointestinal flora and that these mucosal microorganisms make up the major reservoir of pathogens causing pneumonia and many other nosocomial infections, especially in mechanically ventilated patients. Most ventilated ICU patients have a nasogastric tube that provides a direct conduit for reflux of microorganisms from the heavily colonized stomach to the oropharynx, from which organisms gain access to the lower respiratory tract.

SDD consists of four components: (1) a broad-spectrum parenteral antibiotic given for approximately 3 days to treat infections incubating at the time of the admission to the ICU; (2) topical antimicrobials (usually polymyxin E, tobramycin, and amphotericin B) periodically applied to the oropharynx and instilled into the gut for a variable period, usually for the entire duration of ICU stay, to reduce the mucosal burden of gram-negative bacteria and yeasts while preserving the anaerobic flora; (3) a reemphasized adherence to hand hygiene to prevent nosocomial transmission of bacteria, in some European centers, empiric barrier isolation; and (4) serial surveillance cultures of the oropharynx and rectum to monitor the efficacy of the treatment. ${ }^{500,501}$

Eleven meta-analyses assessing the efficacy of SDD for reducing infection and mortality have been published (Table 51-19)..$^{502-512}$ All have found a reduction in pneumonia. Some, but not all, have found reduced mortality. However, a recent review showed that the results of the meta-analyses were inversely related to study design, ${ }^{513}$ which in the case of SDD may overestimate its efficacy. Most studies and meta-analyses of SDD did not make a distinction between parenteral and topical SDD; the few meta-analyses that undertook subgroup analyses found that topical antibiotics alone reduced infection but not mortality. ${ }^{507}$

The greatest deterrent to widespread acceptance of SDD is the fear that it will promote the emergence and spread of antimicrobial resistant microorganisms. Antibiotic pressure is without question the single most powerful force driving the selection of resistant microorganisms and any strategy for prevention of infection in the ICU that has the potential to increase infections caused by multiresistant organisms must be approached very cautiously. A number of studies underlie the concern of promoting antimicrobial resistance with SDD. Numerous studies have documented major shifts in the microbial ecology of the ICU with the use of SDD. ${ }^{514-516}$ In a study by Lingnau and colleagues, ${ }^{514}$ 4.5 years of SDD with ciprofloxacin led to a marked increase in MRSA infection from $17 \%$ to $81 \%$ and of ciprofloxacin-resistant $S$. aureus from $33 \%$ to $80 \%$. The number of infections caused by other multiresistant bacteria such as Acinetobacter was also increased by SDD. ${ }^{514}$

A distinction must also be made between the risk to an individual receiving SDD of infection caused by a resistant pathogen and the institutional risk of an increased prevalence of antimicrobial-resistant organisms related to the use of SDD. Although both consequences are undesirable, given the skyrocketing rates of endemic nosocomial MRSA and VRE infections worldwide, any-however small-potential for increased antimicrobial resistance must be taken seriously. In order to better address this issue, well-designed, cluster-randomized trials that employ multilevel modeling and specifically address the effects of SDD on antimicrobial resistance across the entire spectrum of microbial pathogens at the institutional level are necessary. Until such data are available, we believe that continued North American concerns about the effects of SDD on antimicrobial resistance are justified, particularly in institutions where MRSA and VRE are endemic, which encompasses virtually all larger hospitals. Given that other effective measures for prevention of nosocomial infection exist, we believe that SDD should be restricted to select patients, such as certain trauma patients, or as a potential adjunctive control measure for a nosocomial outbreak caused by multiply resistant organisms. ${ }^{517}$ 
Table 51-19. Meta-Analyses of Randomized Controlled Trials of Selective Digestive Decontamination

\begin{tabular}{|c|c|c|c|c|}
\hline Study, yr & $\begin{array}{l}\text { No. of RCTs } \\
\text { included }\end{array}$ & Description & $\begin{array}{l}\text { Pneumonia Point } \\
\text { Estimate OR or } \\
\text { RR }(95 \% \mathrm{CI})\end{array}$ & $\begin{array}{l}\text { Mortality Point } \\
\text { Estimate OR or } \\
\text { RR }(95 \% \mathrm{CI})\end{array}$ \\
\hline Vandenbroucke, $1991^{502}$ & 6 & Medical and surgical patients & $0.12(0.08-0.19)$ & $0.70(0.45-1.09)$ \\
\hline $\begin{array}{l}\text { SDD Trialists Collaborative } \\
\text { Group, } 1993^{503}\end{array}$ & 22 & Medical and surgical patients & $0.37(0.31-0.43)$ & $0.90(0.79-1.04)$ \\
\hline Kollef, $1994^{504}$ & 16 & Medical and surgical patients & $0.28(0.21-0.38)$ & $0.90(0.74-1.1)$ \\
\hline Heyland et al, $1994^{505}$ & 25 & Medical and surgical patients & $0.46(0.39-0.56)$ & $0.87(0.79-0.97)$ \\
\hline Hurley et al, $1995^{506}$ & 26 & Medical and surgical patients & $0.35(0.30-0.42)$ & $0.86(0.74-0.99)$ \\
\hline $\mathrm{D}^{\prime}$ Amico et al, $1998^{507}$ & 33 & Medical and surgical patients & $0.35(0.29-0.41)$ & $0.88(0.78-0.98)$ \\
\hline Nathens et al, $1999^{508}$ & 21 & Medical and surgical patients & $\begin{array}{r}\text { Medical: } 0.45 \\
(0.33-0.62) \\
\text { Surgical: } 0.19 \\
(0.15-0.26)\end{array}$ & $\begin{array}{r}\text { Medical: } 0.91 \\
(0.71-1.18) \\
\text { Surgical: } 0.70 \\
(0.52-0.93)\end{array}$ \\
\hline Safdar et al, $2004^{509}$ & 4 & Liver transplant patients & $0.88(0.73-1.09)^{*}$ & $0.82(0.22-2.45)$ \\
\hline Liberati et al, $2004^{510}$ & 36 & Medical and surgical patients & $0.35(0.29-0.41)$ & $0.78(0.68-0.89)$ \\
\hline Silvestri et al, $2005^{511}$ & 42 & Medical and surgical patients & $0.30(0.17-0.53)^{\dagger}$ & NR \\
\hline Silvestri et al, $2007^{512}$ & 51 & Medical and surgical patients & $0.73(0.59-0.90)^{\ddagger}$ & $0.80(0.69-0.94)$ \\
\hline $\begin{array}{l}\text { *Overall infection. } \\
\text { †Fungal infections. } \\
\text { "Bloodstream infection. }\end{array}$ & & & & \\
\hline
\end{tabular}

Recent randomized trials have identified several novel measures for prevention of VAP such as semi-recumbent positioning ${ }^{443}$ and subglottic suction endotracheal tubes. ${ }^{518}$ We believe that these approaches are ecologically more attractive control measures for ventilated ICU patients than prophylactic topical and systemic antibiotics.

\section{Pre-emptive Barrier Isolation}

Having fewer patients in a room, improving the facilities for handwashing, and using cohort nursing (i.e., assigning each nurse to designated patients) have reduced the incidence of endemic nosocomial infection in neonatal and pediatric ICUs. ${ }^{169,519}$ Complicated forms of protective isolation have reduced the high rates of nosocomial infection in patients with profound granulocytopenia ${ }^{520}$ or full-thickness burns. ${ }^{169,240}$ Moreover, the routine use of gowns and gloves on a special pediatric unit was associated with a marked decline in the incidence of nosocomial infection with RSV, ${ }^{78}$ and the routine use of gloves for all patient contacts was shown to reduce the incidence of nosocomial $C$. difficile infection nearly fivefold in a large veterans hospital. ${ }^{241}$

Unfortunately, the few studies that have prospectively evaluated protective isolation of ICU patients have been performed in newborns and pediatric patients and have yielded conflicting and generally disappointing results ${ }^{521-524}$; however, most of these studies had major weaknesses in design. ${ }^{243}$ More recently, several studies have shown that pre-emptive use of barrier precautions can effectively reduce the spread of multiresistant organisms such as MRSA or VRE in epidemic ${ }^{525,526}$ and endemic settings (Table 51-20). ${ }^{78,241,522,527-535}$ If colonization by nosocomial organisms could be prevented or at least delayed until invasive devices are removed, the incidence of infection might be significantly reduced.
One major prospective trial that assessed the efficacy of simple protective isolation-which we prefer to call pre-emptive barrier precautions - to reduce the incidence of nosocomial infection during pediatric intensive care studied 70 high-risk children over 30 months who were not immunosuppressed but who required prolonged mechanical ventilatory support and exposure to invasive devices in a pediatric ICU and were randomized to receive standard care without any special precautions or preemptive barrier isolation, with the use of disposable nonwoven polypropylene gowns and nonsterile latex gloves for all patient contacts. $^{527}$ Risk factors predisposing patients to infection were comparable in the two groups. Nosocomial colonization occurred later among isolated patients (median 12 versus 7 days) and was associated with subsequent infection in 2 patients, as compared with 12 patients given standard care. Among children who were isolated, the interval before the first infection was significantly longer (median, 20 versus 8 days), the daily infection rate was twofold lower (86 versus 44 infections per 1000 ICU days), and there were $50 \%$ fewer days with fever. The benefit of isolation was most notable after 7 days of ICU care. Isolation was well tolerated by patients and their families. Unannounced monitoring showed that children in each group were touched and handled indiscernibly by hospital personnel and families.

The study concluded that the use of disposable highbarrier gowns and gloves for the care of select high-risk children who require prolonged ICU care can substantially reduce the incidence of nosocomial infection, is well tolerated, and does not compromise the delivery of care. Simple forms of protective isolation as a general control measure would also seem preferable to attempts to suppress nosocomial colonization with SDD. Further studies 
Table 51-20. Studies of Pre-emptive Barrier Isolation to Contain Spread of Multiresistant Organisms

\begin{tabular}{|c|c|c|c|c|c|c|}
\hline \multirow{4}{*}{$\begin{array}{l}\text { Pre-emptive Barrier } \\
\text { Isolation Precautions for } \\
\text { All High-Risk Patients }\end{array}$} & \multirow{2}{*}{\multicolumn{2}{|c|}{$\begin{array}{c}\text { Control of } \\
\text { Epidemic Spread }\end{array}$}} & \multicolumn{4}{|c|}{ Control of Endemic Infections } \\
\hline & & & \multirow{2}{*}{\multicolumn{2}{|c|}{$\begin{array}{c}\text { Before-After and } \\
\text { Nonrandomized Trials }\end{array}$}} & \multirow{2}{*}{\multicolumn{2}{|c|}{ Randomized Trials }} \\
\hline & \multirow{2}{*}{$\begin{array}{l}\text { No. of } \\
\text { Outbreaks }\end{array}$} & \multirow{2}{*}{$\begin{array}{l}\text { No. }(\%) \\
\text { Totally } \\
\text { Controlled }\end{array}$} & & & & \\
\hline & & & Author & RR $(95 \% \mathrm{CI})$ & Author & RR $(95 \% \mathrm{CI})$ \\
\hline $\begin{array}{l}\text { Methicillin-resistant } \\
\text { Staphylococcus aureus }\end{array}$ & $2^{525}$ & $2(100)^{525}$ & Safdar ${ }^{532}$ & $0.36(0.13-0.98)^{*}$ & No studies & \\
\hline $\begin{array}{l}\text { Vancomycin-resistant } \\
\text { Enterococcus }\end{array}$ & $2^{525,526}$ & $2(100)^{525,526}$ & $\begin{array}{l}\text { Montecalvo }{ }^{534} \\
\text { Slaughter }^{531} \\
\text { Morris }^{535} \\
\text { Srinivasan }^{533} \\
\text { All studies }\end{array}$ & $\begin{array}{l}0.22(0.05-0.92)^{*} \\
2.66(1.00-6.77)^{\dagger} \\
1.18(\mathrm{NR})^{\dagger} \\
0.47(\mathrm{NR})^{*} \\
0.22-2.66\end{array}$ & No studies & \\
\hline $\begin{array}{l}\text { Resistant gram-negative } \\
\text { bacilli }\end{array}$ & none & & McManus $^{614}$ & $0.38(0.31-0.46)^{*}$ & No studies & \\
\hline Clostridium difficile & none & & Johnson ${ }^{241}$ & $0.19(N R)^{*}$ & No studies & \\
\hline \multicolumn{7}{|l|}{ Other } \\
\hline Necrotizing enterocolitis & & & Agbayani ${ }^{522}$ & $0.13(0.02-0.84)^{*}$ & & \\
\hline Respiratory syncytial virus & & & Leclair $^{78}$ & $0.34(0.17-0.60)^{*}$ & & \\
\hline All nosocomial infections & & & Slaughter ${ }^{531}$ & $1.51(0.74-3.12)^{\dagger}$ & $\begin{array}{l}\text { Slota } \\
\text { Klein } \\
528 \\
\text { Koss }^{530}\end{array}$ & $\begin{array}{l}0.48(\mathrm{NR})^{*} \\
0.19 \\
(0.05-0.70)^{*} \\
1.86 \\
(1.10-3.16)^{*}\end{array}$ \\
\hline $\begin{array}{l}{ }^{*} P<0.05 \\
{ }^{\dagger} P>0.05\end{array}$ & & & & & & \\
\hline
\end{tabular}

are necessary to determine the cost effectiveness of prophylactic barrier precautions in the ICU and especially the efficacy of protective isolation in adult surgical ICUs, where the incidence of nosocomial infection is as high as $35 \%$. Studies should also determine the relative importance of wearing a gown, as compared with wearing gloves alone.

Patients with prolonged severe granulocytopenia or those who are receiving high dosages of corticosteroids, usually as part of immunosuppressive regimens to prevent transplant rejection, are at risk for invasive pulmonary infection caused by Aspergillus species, Zygomycetes, and other filamentous airborne fungi, which is associated with high mortality. ${ }^{36-38,536}$ The risk of invasive infection appears to be directly related to the counts of airborne fungi, and numerous outbreaks have been linked to building construction or failure of air-control systems. Studies have shown that the isolation of vulnerable patients in positivepressure rooms with spore-free HEPA-filtered air greatly reduces the risk of invasive infection. ${ }^{36,38}$ HEPA-filtered ICU rooms should be available for the care of patients who have received bone marrow or solid organ transplants and who require intensive care, especially in the early posttransplant period or during the treatment of rejection, when dosages of immunosuppressive drugs are high.

Pre-emptive use of barrier isolation precautions (gowns and gloves) and providing dedicated patient care items such as stethoscopes and sphygmomanometers in all highrisk patients from the time of admission is a simple and effective strategy to prevent HCWs from acquiring hand contamination by multiresistant organisms when having contact with patients with unrecognized colonization or infection and to block transmission to other as yet uncolonized patients.

\section{APPROACH TO A NOSOCOMIAL EPIDEMIC}

As noted earlier, most nosocomial epidemics now occur in ICUs. If an epidemic is suspected, the epidemiologic approach must be methodical and thorough yet expeditious, directed toward establishing the bona fide nature of the putative epidemic infections (i.e., ruling out "pseudoinfections" 242 ); confirming the existence of an epidemic (i.e., ruling out a "pseudoepidemic" ${ }^{242}$ ); defining the reservoirs and modes of transmission of the epidemic pathogens; and, most importantly, controlling the epidemic quickly and completely. Control measures are predicated on accurate delineation of the epidemiology of the epidemic pathogen.

Each hospital, through its infection control committee, must be prepared administratively to carry out an investigation and implement needed control measures. The essential steps in dealing with a suspected outbreak of nosocomial bloodstream infection have been reviewed previously (Box 51-3). ${ }^{143}$ To illustrate the approach to a nosocomial epidemic, the epidemiologic investigation of an unusual and complicated outbreak of infusion-related bacteremia $^{307}$ is reviewed:

During a 2-week period in late March 1985, three patients in a university hospital developed primary nosocomial bacteremia with a similar nonfermentative gram- 
negative bacillus. All three patients had had open-heart surgery between March 11 and March 25 (Fig. 51-13) and became bacteremic 48 to 148 hours after the operation.

The bloodstream pathogen in each case was shown to be Pseudomonas pickettii biovariant 1 . The organism was also cultured from the intravenous fluid of two of the patients at the time because, serendipitously, during the outbreak most adult patients in the hospital receiving intravenous fluids were participating in a study of intra- venous catheter dressings: $:{ }^{57}$ As part of the study protocol, specimens were routinely obtained from patients' intravenous fluid when the catheter was removed. Review of nearly 1000 cultures of intravenous fluid from the infusions of participants in the study since its outset 3 months earlier showed that three additional surgical patients operated on in March had had intravenous fluid cultures positive for $P$. pickettii biovariant 1 (see Fig. 51-13), even though none had shown clinical signs of bacteremia.

\section{Box 51-3}

\section{Evaluation of a Suspected Epidemic of Nosocomial Infections}

Administrative preparedness

Immediately retrieve putative epidemic isolates for confirmation of identity through species and subtyping by one or more methods:

Biotyping

Antimicrobial susceptibility pattern (antibiogram)

Serotyping

Phage-typing

Bacteriocin typing

SDS-PAGE protein electrophoresis

Immunoblot pattern

Multifocus enzyme electrophoresis

Restriction enzyme digestion and restriction fragment polymorphism patterns

DNA probes

Preliminary evaluations and control measures

Identify and characterize individual cases in time, place, risk factors

Strive to identify source of infections

Ascertain whether cases represent true infections, rather than "pseudoinfections"
Ascertain whether cases represent a true epidemic, rather than a "pseudoepidemic"

Develop and implement provisional control measures

Intensify surveillance to detect each new case

Review general infection control policies and procedures

Determine the need for assistance, especially extramural (local, state, CDC)

Epidemiologic investigations

Clinicoepidemiologic studies, especially case-control studies

Microbiologic studies

Develop and implement definitive control measures

Confirm control of epidemic by intensified follow-up surveillance

Report the findings

Intramurally

State Health Department, CDC

Publish the report

Modified from Maki DG, Mermel LA: Infections due to infusion therapy. In Bennett JV, Brachman PS (eds): Hospital Infections, 4th ed. Boston, Lippincott-Raven, 1998.

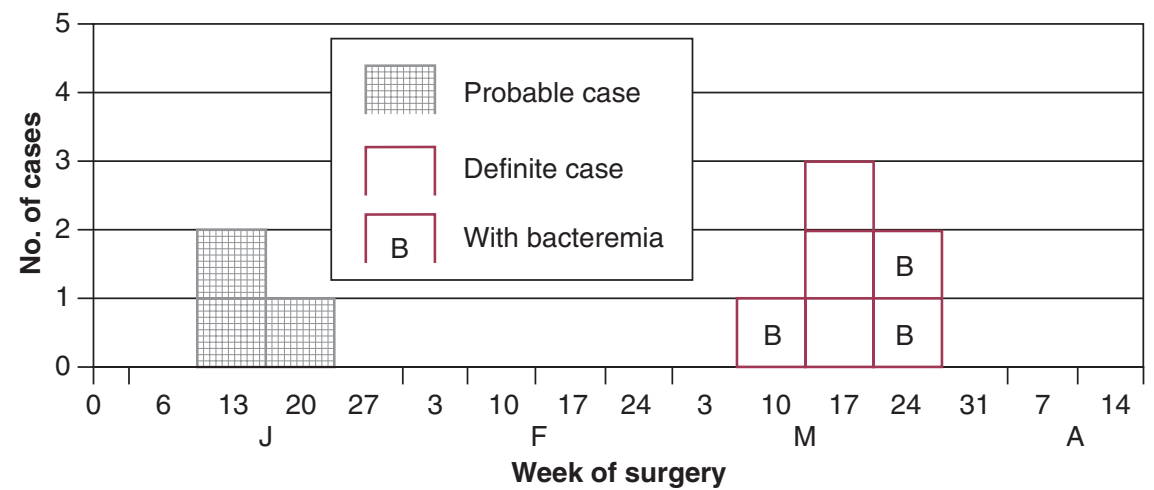

Figure 51-13. Epidemic curve for an outbreak of Pseudomonas pickettii bacteremias and contaminated intravenous infusions traced to contaminated fentanyl given intravenously. Isolates from blood or intravenous fluid of six definite cases (March 1985) were available for reconfirmation and subtyping as $P$. pickettii biovariant 1 ; isolates from intravenous fluid of three probable cases (January 1985) were not available for retesting but were considered likely to have also been $P$. pickettii biovariant 1 on review of the results of 20 biochemical tests common to these three and the six confirmed isolates. (From Maki DG, Klein BS, McCormick RD, et al: Nosocomial Pseudomonas pickettii bacteremias traced to narcotic tampering. A case for selective drug screening of health care personnel. JAMA 1991;265:981-986.) 
Molecular subtyping by restriction enzyme digestion and pulsed-field electrophoresis to delineate restriction polymorphism patterns showed all six isolates to be the clonal. Three more patients who had been operated on in January had had intravenous fluid that cultured positive for a similar nonfermentative gram-negative bacillus; although the three isolates were no longer available, the results of screening by AP-20E biochemical panel (API Analytab, Inc.) at the time were identical to those of the six patients with $P$. pickettii contamination of intravenous fluid, with or without associated bacteremia.

All three septic patients had had multiple positive blood cultures and were clinically in septic shock. P. pickettii had not been isolated from any local site of infection such as the urinary tract, lower respiratory tract, or surgical wound in any of the patients.

Review of nosocomial bacteremias over the past 7 years showed that $P$. pickettii had not previously been identified in any positive blood cultures from the institution, indicating that the cluster of three cases and six instances of contaminated infusate without bacteremia represented a true epidemic and, with the results of the subtyping, a common source epidemic.

The CDC and the manufacturer were contacted: None of more than 70 NNIS hospitals had reported $P$. pickettii bacteremias in the past year, and the manufacturer had never identified contamination with $P$. pickettii in quality control microbiologic sampling of its fentanyl before distribution or received any complaints from users about suspected contamination of their fentanyl. Moreover, a survey of surrounding Wisconsin hospitals that also used the manufacturer's fentanyl revealed none experiencing nosocomial bacteremias with $P$. pickettii.

A case-control study comparing the 9 cases, all of whom had had recent surgery, with 19 operated patients who had had negative intravenous fluid cultures in the intravenous dressing study (Table 51-21), showed that all 9 cases but only 9 of the 19 operated control cases had received fentanyl intravenously in the operating room $(P=.05$; the mean total dose given to the 9 cases was far greater than that given to control patients who received the drug, $3080 \mu \mathrm{g}$ versus $840 \mu \mathrm{g}, P<.001)$.

In the hospital at the time, fentanyl was used only in the operating rooms as part of balanced anesthesia. The drug was received in $20-\mathrm{mL}$ ampules from the manufacturer, and each week one of three pharmacy technicians, by rotation, drew into sterile syringes all fentanyl likely to be needed the following week in the operating rooms. Each day, one of the technicians delivered enough predrawn syringes to the operating rooms to meet the needs of the cases being done that day. Cultures of predrawn fentanyl in syringes in the central pharmacy, prompted by the findings of the case-control study, showed that twenty $(40 \%)$ of fifty $30-\mathrm{mL}$ syringes sampled were contaminated by $P$. pickettii, in a concentration of greater than $10^{4} \mathrm{CFU} / \mathrm{mL}$; none of thirty-five 5 - or $2-\mathrm{mL}$ syringes showed contamination $(P=.001)$.

Extensive culturing within the central pharmacy was negative for evidence of environmental contamination by $P$. pickettii with one exception: $P$. pickettii biovariant 1 ,
Table 51-21. Case-Control Analysis of Risk Factors for Bacteremia or Contaminated Intravenous Fluid with Pseudomonas Pickettii

\begin{tabular}{|c|c|c|c|}
\hline & $\begin{array}{l}\text { Cases } \\
(n=9)\end{array}$ & $\begin{array}{l}\text { Controls* } \\
(n=19)\end{array}$ & $P$ Value \\
\hline Age, Mean & $50 \mathrm{yr}$ & $46 \mathrm{yr}$ & $N S^{\dagger}$ \\
\hline Duration of Surgery, Mean & $4.0 \mathrm{hr}$ & $3.7 \mathrm{hr}$ & NS \\
\hline $\begin{array}{l}\text { Type of Surgery } \\
\text { Cardiovascular } \\
\text { General }\end{array}$ & $\begin{array}{l}5(55) \\
4(45)\end{array}$ & $\begin{array}{r}3(16) \\
16(84)\end{array}$ & NS \\
\hline $\begin{array}{l}\text { Intravenous Fluids } \\
\text { Lactated Ringer's } \\
\text { Dextrose in Ringer's } \\
\text { lactate } \\
\text { Saline } 0.9 \% \\
\text { Blood products } \\
\text { Albumin, fresh frozen } \\
\text { plasma }\end{array}$ & $\begin{array}{l}8(89) \\
5(55) \\
6(67) \\
7(78) \\
4(44)\end{array}$ & $\begin{array}{l}11(58) \\
14(74) \\
4(21) \\
4(21) \\
2(10)\end{array}$ & $\begin{array}{l}\text { NS } \\
\text { NS } \\
\text { NS }\end{array}$ \\
\hline $\begin{array}{l}\text { Intraoperative Intravenous } \\
\text { Pentothal } \\
\text { Lidocaine } \\
\text { Pancuronium } \\
\text { Heparin } \\
\text { Cefazolin } \\
\text { Fentanyl } \\
\text { Volume of intravenous } \\
\text { fentanyl, mean }\end{array}$ & $\begin{array}{l}\text { edications } \\
4(45) \\
5(55) \\
5(55) \\
5(55) \\
9(100) \\
9(100) \\
61.6 \mathrm{~mL}\end{array}$ & $\begin{aligned} & 13(68) \\
& 5(26) \\
& 4(21) \\
& 0(0) \\
& 10(53) \\
& 9(47) \\
& 16.8 \mathrm{~mL}^{\ddagger}\end{aligned}$ & $\begin{array}{l}\text { NS } \\
\text { NS } \\
\text { NS } \\
<.001 \\
\text { NS } \\
.05 \\
<.001\end{array}$ \\
\hline \multicolumn{4}{|c|}{$\begin{array}{l}\text { *Patients randomly selected who had had surgery on the same day as } \\
\text { cases but who had negative cultures of intravenous fluid from their } \\
\text { infusion begun in the operating room. } \\
\text { 'Not significant at } P<.05 \text {. } \\
\text { 'For the nine control patients who received fentanyl. } \\
\text { From Maki DG, Klein BS, McCormick RD, et al: Nosocomial } \\
\text { Pseudomonas pickettii bacteremias traced to narcotic tampering. A case } \\
\text { for selective drug screening of health care personnel. JAMA } \\
1991 ; 265: 981-986 \text {. }\end{array}$} \\
\hline
\end{tabular}

with an identical antimicrobial susceptibility pattern and restriction enzyme fragment pattern to the epidemic strain recovered from blood cultures or patients' intravenous infusions, was cultured in a concentration of 28 to $80 \mathrm{CFU} /$ $\mathrm{mL}$ from five specimens of distilled water drawn from a tap in the central pharmacy. The epidemic strain was shown to multiply well in the fentanyl solution, attaining concentrations exceeding $10^{4} \mathrm{CFU} / \mathrm{mL}$ within 48 hours.

A second case-control study suggested strongly that the epidemic was caused by theft of fentanyl from 30-mL syringes by one pharmacy staff member and replacement by distilled water that the individual thought was sterile but which, unfortunately, was contaminated by $P$. pickettii. The pharmacy member resigned early in the investigation and no longer works in the hospital. On April 29, 1985, the hospital's system for providing fentanyl and other narcotics to the operating rooms was changed; narcotics are no longer predrawn into syringes in the central pharmacy but are delivered to the operating rooms in unopened vials or ampules, and anesthesiologists' orders for narcotics are filled by a staff pharmacist assigned to the operating room. No further bacteremias with $P$. pickettii have occurred since March 25, 1985 (see Fig. 51-13), and cultures of more than 6000 samples of hospitalized patients' 
intravenous fluid in research studies since then have shown no further contamination by $P$. pickettii.

This outbreak illustrates the power of genetic subtyping ${ }^{147}$ and case-control analyses to identify the cause of an epidemic. It further illustrates the potential for contamination of parenteral drugs or admixtures and the extraordinary range of epidemiologic mechanisms of nosocomial bloodstream infection deriving from such contamination. ${ }^{107}$

If epidemiologic or microbiologic studies suggest or indicate intrinsic contamination of a widely distributed commercial product or device, the local and state health authorities, U.S. Food and Drug Administration, CDC, and manufacturer should be informed immediately. Remaining products should be quarantined and retained for evaluation by the public health authorities.

\section{PROTECTION OF HEALTH CARE WORKERS IN THE INTENSIVE CARE UNIT}

HCWs in general, but especially those working in ICUs who are exposed daily to critically ill patients, many of whom have contagious but undiagnosed infections, are at increased risk of acquiring occupationally related infections including tuberculosis; herpes simplex virus infection; chickenpox; cytomegalovirus infection; hepatitis A, $\mathrm{B}$, or $\mathrm{C}$; HIV infection; influenza; measles; rubella; mumps; pertussis; and viral conjunctivitis. ${ }^{538-540}$

Fortunately, the risk posed by many of these infections can be eliminated by immunization of the HCW. ${ }^{541}$ The Advisory Committee on Immunization Practices (ACIP) last made recommendations for the vaccination of HCWs in $1997^{542}$; however, a number of new developments in vaccine-preventable diseases have emerged since these guidelines were last published, and a considerable number of occupational infections exist for which there are currently no vaccines.

\section{General Precautions against Biohazardous Exposure}

All hospitals, as mandated by JCAHO,${ }^{31}$ must have an employee health service and written protocols for the management of biohazardous exposures (Box 51-4). ${ }^{125,543}$ Such protocols permit expeditious and comprehensive evaluation and timely administration of postexposure prophylaxis (PEP) with antiretrovirals after HIV exposure, immune serum globulin or hepatitis B immune globulin after exposure to hepatitis A or B, or antimicrobial prophylaxis after exposure to contagious bacterial pathogens such as Neisseria meningitidis or Bordetella pertussis.

The single greatest measure a hospital can take to reduce the risk of biohazardous exposures is to implement a comprehensive program that actively monitors occupational exposures, ${ }^{544}$ educates HCWs about the consequences and ways to prevent exposures, ${ }^{545}$ and advocates for and rigorously evaluates new safety technologies as a means of reducing exposures further. ${ }^{546}$

The importance of uncompromising compliance with universal precautions to protect HCWs from unknowing exposure to HIV and other bloodborne viruses has been discussed. Importantly, the greatest emphasis in training on universal precautions should be placed on measures to prevent needle sticks and other sharps injuries, ${ }^{543}$ prohibition of recapping used needles, and making impervious needle disposal containers ${ }^{547}$ available at the bedside of each ICU patient. ${ }^{548}$ The use of engineered controls such as needleless systems holds the greatest promise for effecting a material reduction in hazardous sharps injuries. ${ }^{546}$

\section{Vaccine-Preventable Diseases}

\section{Hepatitis $B$}

Percutaneous exposure of an unvaccinated HCW to blood from a patient who is hepatitis B surface antigen (HBsAg) positive carries considerable risk of seroconversion $(23 \%$ to $37 \%)$ and development of clinical disease ( $1 \%$ to $6 \%) .{ }^{125}$ This risk is greatly magnified if the source patient is hepatitis $\mathrm{B}$ e antigen ( $\mathrm{HBeAg}$ ) positive: the risk of seroconversion is $37 \%$ to $62 \%$, and the risk of developing clinical disease is $22 \%$ to $31 \% .{ }^{125}$ The introduction of the recombinant hepatitis B vaccine in 1986 has greatly reduced the risk of occupationally acquired disease, and it is recommended that all HCWs receive a complete series of immunizations at 0,1 , and 6 months. ${ }^{542}$ Furthermore, ACIP recommends that all HCWs have documentation of protective serum titers (hepatitis B surface antibody [HBsAb] serum titer $=10 \mathrm{IU} / \mathrm{mL}$ ) 1 to 2 months after completion of their primary series. Those HCWs who do not mount protective titers should undergo a secondary immunization series. ${ }^{542} \mathrm{HCWs}$ exposed to hepatitis B who have not developed protective titers after a primary or secondary immunization series or who have not completed a primary immunization series should be given postexposure prophylaxis (PEP) as detailed by the U.S. Public Health Service. ${ }^{125}$

\section{Measles, Mumps, and Rubella}

Reported cases of measles, mumps, and rubella have declined steadily in the United States since the introduction of mandatory childhood immunizations. ${ }^{549-551}$ However, epidemics of measles and, more recently, mumps, continue to be a problem in many parts of the country. ${ }^{552,553}$ Severe measles in susceptible HCWs who themselves exposed additional patients while in the incubation phase of infection have been well described in the literature. ${ }^{73,554}$ As a result, it is recommended that all HCWs born after 1956 have documented immunity to measles and rubella by either (1) laboratory evidence of a protective titer; (2) physician documentation of clinical measles and rubella; or (3) documentation of appropriate vaccination against measles and rubella. ${ }^{542}$ Although ACIP is less stringent with regards to documentation of immunity to mumps, it is recommended that HCWs receive the trivalent measles, mumps, rubella (MMR) vaccine rather than single vaccines. ${ }^{542}$ When nonimmune HCWs are exposed to measles, PEP with MMR vaccination has been shown to reduce the likelihood of developing fulminant disease ${ }^{555}$ Measles immunoglobulin is another PEP option for nonimmune HCWs who have a contraindication to immunization-pregnancy—but only provides temporary protection. ${ }^{555}$ 


\section{Guidelines for Prevention and Management of Biohazardous Injuries in the Hospital*}

1. Hospital personnel must be made more personally aware of the potential catastrophic sequelae of sharps injuries and other biohazardous exposures and of guidelines for prevention. This must be conveyed in initial orientation programs for all new personnel including physicians and by periodic updates on each patient care unit and presentations at staff conferences. The topic can also be periodically reviewed in the hospital's newsletter for personnel. Prominently placed posters warning of the hazards of sharps injuries and listing simple precautions to avert such injuries, especially in areas where sharps are heavily used, may also be of value.

2. Sharps disposal units should be made widely and conveniently available throughout the hospital, especially in locations that facilitate their immediate use: in individual patient rooms, nursing stations, pharmacy units, and utility rooms; in all clinical laboratories; on anesthesia carts; and in each operating room. Receptacles should be made of impervious material such as plastic or metal and should be emptied according to an established routine by personnel who have been properly instructed. Disposal units should consist of impervious receptacles into which a used needle and syringe or other sharp can be immediately dropped without handling it further. If it is necessary to remove a used needle from a syringe or Vacutainer, an instrument should always be used.

3. Recapping or resheathing of used needles must be strongly discouraged except when rare circumstances do not permit immediate disposal, in which case capping must be done using a "one-handed technique" or special sheath.

4. Medical or nursing personnel must be apprised of the importance of obtaining adequate assistance when administering injections or infusion therapy to patients who may not be able to cooperate.

5. Personnel must be apprised of the need to use extreme care in cleaning up after procedures that involve needles or other sharps such as lumbar punctures, thoracentesis, or central venous catheter placement.

6. A strong institutional commitment to the continuous evaluation and selective adoption of technology-based approaches to prevention of sharps injuries will have the greatest impact.

7. With accidental sharps injuries, unless the injury occurred with a clean sharp not used on a patient, the injury should be immediately reported to the employee health service, where work-related injuries in general can be evaluated and managed most consistently and inexpensively and surveillance of all work-related injuries can be assisted. A mechanism to ensure prompt management of biohazardous injuries 24 hours a day (including during nights and weekends by emergency department personnel who have been trained in the institutional biohazardous injury protocol) must be available.
All hospital personnel who render care or services to patients including all staff physicians, house officers, and health care students should be covered by this institutional service to ensure consistent reporting and treatment of injuries.

Employee health service personnel should meet with each employee sustaining a biohazardous injury, ascertain the exact reason for the accident, and review how to prevent a similar injury in the future. Particular attention should be given to those HCWs who have sustained repeated injuries to identify accidentprone activities or individuals or high-risk work situations that should be modified.

8. Institutions should carry out continuous surveillance and reporting of all biohazardous injuries, which can form the basis for preventive programs and determine their effectiveness. Risk management personnel should include sharps injury reduction as a major institutional priority.

9. Management of sharps injuries: It is beyond the scope of this review to provide a comprehensive protocol for management of sharps injuries; however, it is imperative that all hospitals have a protocol that provides unambiguous guidelines for management, specifically the following:

a. Clear definitions of biohazardous injuries

b. Procedures for immediate care of the injury at the time of occurrence (e.g., squeezing the puncture wound to induce bleeding, disinfection with a virucidal agent, such as an iodophor)

c. Procedures to determine expeditiously the magnitude of risk (i.e., screening the source patient for evidence of active infection by hepatitis $\mathrm{B}$ virus; hepatitis $\mathrm{C}$ virus; and HIV). With regard to HIV screening, the protocol must be in compliance with state statutes governing HIV testing.

d. The responsibilities of the injured HCW; the worker's supervisor; the employee health service; and, after working hours, the emergency department

e. Guidelines for postexposure immunoprophylaxis and drug therapy, especially with exposures to the following:

Hepatitis A, B, and C

HIV

Jakob-Creutzfeldt disease

Syphilis

Malaria

Bacteremia

f. Follow-up of the exposed HCW, especially following exposures to HIV; hepatitis B virus; and hepatitis $C$ virus

g. Administrative follow-up of all injuries, to minimize recurrences, and institutional surveillance of biohazardous injuries

h. Periodic review of the protocol, with revision as indicated

*The Biohazardous Exposure Protocol of the University of Wisconsin Hospital and Clinics is available from the authors on request. Modified and updated from McCormick RD, Meisch MG, Ircink FG, Maki DG: Epidemiology of hospital sharps injuries: A 14-year prospective study in the pre-AIDS and AIDS eras. Am J Med 1991;91:301S-307S. 


\section{Varicella}

Occupational acquisition of varicella-zoster virus (VZV) is well described, with transmission rates among susceptible HCWs ranging from $2 \%$ to $16 \%$ in published studies. ${ }^{511,556}$ Infected HCWs pose a threat for transmission to other susceptible patients, and HCWs, when compared with persons who acquire varicella during adolescence, often experience more severe disease, ${ }^{557}$ particularly if pregnant. ${ }^{558}$ All HCWs should be asked about a history of primary varicella. In the absence of a positive history, all HCWs should undergo serologic testing to confirm seropositivity or be immunized with the live, attenuated vaccine. ${ }^{559}$ Susceptible HCWs should not care for patients with suspected or confirmed varicella or zoster infections, particularly if pregnant. In the event of an accidental exposure, susceptible HCWs should undergo varicella immunization within 72 hours of exposure on the basis of studies demonstrating a reduced risk of developing clinical infection and attenuated clinical disease. ${ }^{560}$ The routine use of varicella-zoster immune globulin (VZIG) for PEP of susceptible HCWs exposed to VZV is not recommended. ${ }^{559}$ Rather, the decision to administer VZIG should be guided by the presence of risk factors that increase the risk of severe disease (e.g., pregnancy or other immunodeficient conditions such as advanced AIDS).

\section{Influenza}

Influenza is responsible for 114,000 hospitalizations and 36,000 deaths in the United States each year. ${ }^{561}$ Nosocomial outbreaks of influenza are common and serve to put HCWs at considerable risk of acquiring and transmitting influenza to others. ${ }^{562,563}$ These outbreaks impose considerable economic burden on health care institutions and increase adverse events among HCWs and patients alike. ${ }^{564}$ Influenza immunization of HCWs has significantly reduced days of febrile illness and sick leave in randomized controlled trials, ${ }^{565,566}$ and surveys suggest that HCWs tend to view influenza immunization as a means of protecting themselves from illness. ${ }^{567,568}$ However, accumulating evidence suggests that influenza immunization of HCWs also significantly reduces rates of influenza-related morbidity and mortality among potentially exposed patients in a variety of health care settings. ${ }^{569-571}$ Moreover, expanding influenza rates of HCWs has been shown to be cost saving from an institutional perspective. ${ }^{572}$ Despite this evidence, voluntary immunization rates among U.S. HCWs remain disappointingly low-in the range of $40 \%{ }^{573}$ For these reasons, some experts are beginning to call for mandatory influenza immunization of HCWs (i.e., a condition of employment) in much the same way that hepatitis B and MMR vaccinations are mandatory for $\mathrm{HCWs}$ in most hospitals. ${ }^{574}$

\section{Pertussis}

HCWs are at increased risk of developing pertussis as a result of waning immunity in adulthood, ${ }^{575}$ and numerous pertussis epidemics among HCWs have been reported in recent years. ${ }^{576,577}$ Pertussis outbreaks are associated with considerable economic consequences, ${ }^{577,578}$ and studies have shown that routinely providing a pertussis booster to HCWs in the form of tetanus toxoid, reduced diphtheria toxoid, and acellular pertussis adsorbed vaccine (Tdap) is cost effective. ${ }^{577}$ The National Foundation of Infectious Diseases (NFID) and ACIP have recommended that all HCWs undergo booster immunization with Tdap, although these recommendations have not yet been endorsed by the U.S. Department of Health and Human Services. ${ }^{579}$

\section{Non-Vaccine-Preventable Diseases of Major Interest}

\section{Herpetic Whitlow}

Primary HSV paronychia has long been an occupational hazard of being an ICU nurse or physician ${ }^{580,581}$ and is usually associated with a great deal of pain and discomfort, fever, and lymphadenitis. Leave from work for 2 to 3 weeks is not uncommon. One of the most compelling reasons for ICU HCWs to routinely wash their hands after contact with an ICU patient and to wear gloves during any contact with a patient's airway-including gloves on both hands during tracheal suctioning-is to protect themselves from this miserable condition.

\section{Tuberculosis}

Numerous reports of tuberculosis (TB) outbreaks in hospitals and other health care settings in the early and mid1990s reminded the health care community of the risk associated with the care of patients with active pulmonary tuberculosis. ${ }^{70-72,234}$ The feature common to most of these outbreaks was failure to recognize patients with active pulmonary tuberculosis. The most important measure for preventing the transmission of tuberculosis is to (1) rapidly identify and isolate patients with suspected tuberculosis and (2) promptly initiate appropriate therapy when tuberculosis is confirmed, to reduce the duration of infectiousness. ${ }^{582}$ Updated guidelines for the prevention of nosocomial TB have recently been published that stress the importance of administrative, environmental, and personal respiratory protection controls. ${ }^{126}$ Implementation of these recommendations has led to remarkable reductions in nosocomial transmission rates. ${ }^{235}$

\section{Hepatitis $C$}

Nearly 4 million persons in the United States are infected with hepatitis $\mathrm{C}$ virus (HCV), which is responsible for 8000 to 10,000 deaths every year. ${ }^{583}$ Although the already high and rising prevalence of $\mathrm{HCV}$ infection in the population potentially exposes $\mathrm{HCW}$ to occupationally acquired infection, most studies have failed to find a higher seroprevalence to HCV among HCWs than in the general population. ${ }^{584}$ However, at least one study found that a previous history of a needle stick injury is associated with an increased risk of $\mathrm{HCV}$ infection, ${ }^{585}$ and pooled analyses of published studies suggest that the seroconversion rate following a percutaneous injury involving HCV-positive persons is about $0.5 \%$, a risk that is similar to that seen with percutaneous injuries involving needle stick exposures to HIV. ${ }^{584}$ Current guidelines for the management of HCWs exposed to HCV only recommend serologic 
testing and measurement of serum alanine aminotransferase (ALT) levels, with or without HCV PCR testing, at baseline and again 4 to 6 months later, and do not make specific treatment recommendations. ${ }^{125}$ However, these recommendations may undergo modification in the near future in light of studies demonstrating sustained virological responses to interferon monotherapy in excess of $90 \%$ in patients with acute-onset HCV infection. ${ }^{586,587}$

\section{Human Immunodeficiency Virus}

Although the risk of occupationally acquired HIV infection is low, it is not zero. As of 2002, there have been 57 probable and an additional 139 possible cases of occupationally acquired HIV in the United States. ${ }^{58}$ The vast majority involved percutaneous injuries from hollow-bore needles contaminated with blood. ${ }^{584}$ Other types of sharps injuries are less likely to transmit HIV, and only a single documented case of occupational HIV transmission caused by a body fluid exposure other than blood-bloody pleural fluid-has been reported in the literature. ${ }^{589}$ Although no randomized trials exist, there are considerable data from animal $^{590}$ and observational studies ${ }^{591}$ to suggest that PEP with antiretrovirals can significantly reduce the likelihood of HIV transmission following a high-risk exposure. For this reason, the U.S. Public Health Service has issued detailed recommendations on occupational HIV PEP with the choice and number of drugs driven by characteristics of the HCW, type of exposure, and characteristics of the source patient. ${ }^{592}$

\section{GOALS FOR THE FUTURE}

Clearly, nosocomial infection is one of the most important causes of iatrogenic morbidity and mortality in patients who require prolonged life-support care in an ICU. Much has been learned over the past 3 decades about the relative risks and especially the pathogenesis and epidemiology of these infections, information that has provided the scientific underpinnings for preventive strategies that have proved effective. However, there is an urgent need for better research to prevent nosocomial infection in ICU patients (Box 51-5), ${ }^{593}$ particularly with respect to strategies to prevent colonization by multiresistant microorganisms, and to prevent infection even if colonization has already occurred. ${ }^{28}$

Most of our understanding of the epidemiology of nosocomial infection, especially in ICUs, is based on studies of epidemics. Well-designed studies are necessary to better define the epidemiology of endemic nosocomial infections, especially those caused by resistant staphylococci, enterococci and gram-negative bacilli, and yeasts. The importance of hand carriage of pathogens by hospital personnel, the role of airborne transmission in the ICU, and the relevance of contamination of the inanimate hospital environment by resistant pathogens all need to be better delineated, as well as the factors influencing nosocomial colonization and superinfection by resistant bacteria and yeasts.

In addition, larger and more sophisticated studies, using multivariate techniques of statistical analysis to define risk factors for the major forms of nosocomial infection in the ICU, are necessary to guide allocation of infection control resources and to target future research efforts.

Considering that the period of greatly increased susceptibility to infection of ICU patients is limited-until the invasive devices have been removed-a major commitment must be made to devise and evaluate strategies for blocking transmission of organisms between patients and preventing, or at least delaying, nosocomial colonization.

One of the oldest yet most important infection control measures-hand hygiene-is still done almost indifferently by HCWs in most hospitals including within ICUs. Innovative approaches are necessary to improve the frequency and the quality of handwashing after patient contacts likely to result in acquisition of nosocomial organisms. Exactly how should hands be washed for maximal benefit and with what agents? The question can be posed: Could very frequent handwashing, which approaches 40 times per 8-hour shift in neonatal ICUs, increase the potential for transmission of microorganisms, such as methicillinresistant coagulase-negative staphylococci? Should the frequency of handwashing, as well as the agents used, be critically reexamined? Beyond a certain frequency, more may not necessarily be better. Should handwashing machines, which substantially augment degerming, ${ }^{594,595}$ be adopted widely? Could regular application of chlorhexidine-containing evaporative lotions, used without water, replace some of the conventional handwashing or at least compensate for the suboptimal handwashing currently practiced? ${ }^{221,596,597}$ Large clinical trials, ideally in multiple centers, are necessary to ascertain the efficacy or lack of efficacy of innovative approaches to hand degerming in reducing infections in high-risk patients, particularly in nurseries and ICUs.

Whereas SDD with topical nonabsorbable antibiotics has shown promise for the prevention of nosocomial respiratory infection in ICU patients, as noted, the potential effect on the microbial ecology of the ICU must be viewed with caution, and the cost-benefit and long-term effects of SDD need better clarification. The uses of simple barrier precautions to prevent colonization and infection have shown promise and warrant further study, especially in ICUs.

Studies have shown that the use of dedicated intravenous therapy teams, consisting of trained nurses or technicians to ensure a high level of aseptic technique during catheter insertion and in follow-up care of the catheter, has been associated with greatly reduced rates of catheterrelated infection and appears to be cost effective. ${ }^{322,323,598}$ The use of teams of trained ICU personnel to insert all urethral catheters and provide follow-up care for these catheters, all intravascular devices, and percutaneous tubes in the ICU deserves study.

Remarkably, there have been few comparative clinical trials of the various chemical antiseptics available for disinfecting skin before inserting intravascular devices or assisting in surgery or studies of antiseptic handwashing agents. Large, randomized clinical trials, ideally in multiple centers, are necessary in which infection, rather than 


\section{Box 51-5}

\section{Directions for Future Research in Nosocomial Infection Control}

Studies to better define the epidemiology of endemic nosocomial infections:

Especially those caused by resistant staphylococci, gram-negative bacilli, and Candida

The relevance of hand carriage of pathogens by hospital personnel

The role of airborne transmission

The relative importance of contamination of the inanimate hospital environment, especially with methicillin-resistant $S$. aureus, $C$. difficile, and other resistant organisms

The biologic factors influencing colonization by nosocomial organisms

The factors governing superinfection by resistant bacteria and Candida

Better understanding of risk factors predisposing to infection, especially in the ICU, to guide allocation of resources in infection control and focus research efforts

Innovative strategies to prevent nosocomial colonization and interrupt cross-infection, especially in ICUs:

New approaches to improving compliance with and improving the effectiveness of handwashing between patients

Various types of barrier precautions (forms of protective isolation)

The true efficacy and cost-benefit of selective digestive decontamination; the ecologic effects of longterm use must be assessed carefully
Dedicated device-care teams

Large, randomized clinical trials of the various cutaneous antiseptics available, with infection, rather than colonization, as the index of comparison, for handwashing by personnel, site disinfection with invasive devices, patient bathing, and decolonization

Research on devices:

Innovative designs to implicitly reduce contamination

Colonization-resistant polymers, possibly incorporating antimicrobials onto the surface or into the polymer itself

Better techniques of use to enhance safety

Cost-effective "needleless" systems to protect health care personnel

Improved laboratory tests to identify infection more accurately and rapidly, especially tracheobronchitis and pneumonia, to reduce unnecessary antibiotic therapy yet permit early therapy to avert progression to life-threatening sepsis

Measures to restrict and improve the use of antibiotics, especially in ICUs

Expanded, more effective approaches to education in infection control for health care personnel, especially physicians, with respect to handwashing, use of isolation, invasive devices, and use of antibiotics

Modified from Maki DG: Risk factors for nosocomial infection in intensive care. "Devices vs. nature" and goals for the next decade. Arch Intern Med 1989;149:30-35.

cutaneous colonization or positive cultures, is used as the index of comparison.

Considerable evidence indicates that the material used in construction of an implanted device plays an important role in the pathogenesis of device-related infection, namely, whether the material provides an attractive surface for adherence by pathogenic microorganisms such as coagulase-negative staphylococci. Studies are necessary to delineate fully the molecular mechanisms of microbial adherence to prosthetic surfaces to develop new materials intrinsically resistant to colonization for use with implantable devices and to design devices that intrinsically deny microbial access.

Increased use of diagnostic tests has greatly increased awareness of infectious diseases. Improved laboratory techniques to identify infection more accurately and rapidly, especially methods to reliably distinguish colonization of the lower respiratory tract from early infection that merits antimicrobial therapy, could greatly reduce unnecessary antimicrobial therapy yet detect infections earlier, before they progress to sepsis with multiple-organ failure.

Antimicrobials are not used optimally in most ICUs, and there is much overuse in hospitals, particularly of extended-spectrum penicillins and cephalosporins, imipenem, and quinolones. Antibiotic pressure has had a powerful effect on the hospital microbial ecology and, as noted previously, on the profile of nosocomial infection, especially in ICUs. We must and can do better.

Last, but certainly not least, many physicians remain remarkably oblivious to the most basic precepts of infection control, and nurses are in general far better informed and are a more effective force for ensuring compliance with infection control practices. More effective ways to communicate essential information on nosocomial infection control to hospital personnel, especially with regard to handwashing, aseptic use of devices, and antibiotic therapy, and to apply it more consistently in all hospitals, would have vast immediate benefits. 


\section{KEY POINTS}

- Patients in modern-day ICUs experience rates of nosocomial infection three to five times higher than non-ICU hospitalized patients. Rates of primary bacteremia and nosocomial pneumonia are up to 10 times higher.

- Patients who are severely immunocompromised or who are critically ill and have high severity of illness scores have a substantially increased risk of nosocomial infection. However, most nosocomial infections in the ICU appear causally to be most directly related to lifesaving technology, particularly invasive devices such as endotracheal tubes and mechanical ventilatory support, urethral and intravascular catheters, and intraventricular catheters, which facilitate colonization by nosocomial organisms and greatly increase vulnerability to infection.

- The major reservoir of bacterial nosocomial pathogens, and possibly Candida as well, in the ICU is the colonized or infected patient. Most infections begin with nosocomial colonization by organisms acquired from the hands of noncolonized HCWs. Increasing evidence suggests that antibiotic-resistant organisms, particularly MRSA, resistant enterococci, and C. difficile, may also be acquired from the inanimate environment immediately surrounding the patient. Mycobacterium tuberculosis, Legionella, Aspergillus, influenza A virus, varicella-zoster virus, measles, mumps, and the new highly virulent SARS human coronavirus are transmitted by the airborne route.

- ICUs are uniquely conducive to the epidemic spread of nosocomial organisms of all types, especially antibioticresistant bacteria and even Candida; more than half of all hospital epidemics occur in ICUs.

- An active, visible institutional infection control program can prevent up to one third of nosocomial infections. Surveillance of infection, whether total or focused, and education of all personnel are the most essential components of the program.

- Use of a chlorhexidine-containing agent for handwashing between patients will reduce endemic nosocomial infections in the ICU by at least $30 \%$. The regular use of waterless alcohol-containing hand rubs or gels may provide comparable benefit in prevention of cross-infection.

- Stringent attention to isolation precautions, especially disposable gloves and a gown for contacts with patients known to be infected or colonized by resistant organisms, is mandatory to minimize cross-infection and prevent outbreaks. Misuse of gloves as part of universal precautions may, paradoxically, increase the risk of nosocomial infection.
- Modern-day ICUs must have adequate numbers of special negative-pressure isolation rooms for the care of patients with suspected or proven pulmonary tuberculosis and other airborne infections, such as varicella-zoster virus.

- Patients who have undergone recent bone marrow transplants or who have received intensive chemotherapy and are experiencing prolonged severe granulocytopenia should receive ICU care in special HEPA-filtered positive-pressure isolation rooms to protect them from devastating deep Aspergillus and other filamentous fungal infection.

- Meticulous attention to aseptic technique and the use of maximal barrier precautions-long-sleeved sterile surgical gown, mask and head cover, as well as sterile glovesduring the insertion of central venous catheters; the use of $2 \%$ chlorhexidine solutions for cutaneous antisepsis; avoiding insertion into the femoral veins; and prompt removal of catheters as soon as they are no longer necessary can reduce the incidence of catheter-related bloodstream infection at least threefold.

- Studies suggest that the prophylactic use of simple barrier precautions, vis-à-vis protective isolation, may provide protection against all types of ICU-acquired infection. Protective isolation is more appealing ecologically than the use of SDD.

- Measures to avert needle sticks and other sharps injuries are the most important aspect of universal precautions; these measures are necessary to protect the HCWs from HIV and other bloodborne viruses.

- A crisis of antibiotic resistance exists in ICUs. The progressive increase in antibiotic resistance of nosocomial staphylococci (methicillin), gram-negative bacilli (aminoglycosides and expanded-spectrum betalactams), and enterococci (vancomycin or ampicillin), and the sixfold increase in Candida infections during the past 2 decades indicates that it is of highest priority to reduce antimicrobial pressure within ICUs.

- Novel technology holds the greatest promise for prevention of nosocomial infection in general, particularly the development of medical devices that are intrinsically resistant to infection.

- Identifying more effective ways to communicate knowledge of infection control to hospital personnel, especially with regard to handwashing, aseptic use of devices, and antibiotic therapy, and to apply it consistently in all hospitals would have vast immediate benefits.

\section{REFERENCES}

1. Knaus WA, Draper EA, Wagner DP Zimmerman JE: An evaluation of outcome from intensive care in major medical centers. Ann Intern Med 1986;104:410-418.

2. Reynolds HN, Haupt MT, ThillBaharozian MC, Carlson RW: Impact of critical care physician staffing on patients with septic shock in a university hospital medical intensive care unit. JAMA 1988;260:3446-3450.

3. Ron A, Aronne LJ, Kalb PE, et al: The therapeutic efficacy of critical care units. Identifying subgroups of patients who benefit. Arch Intern Med 1989;149:338-341.

4. Brown RB, Hosmer D, Chen HC, et al: A comparison of infections in different
ICUs within the same hospital. Crit Care Med 1985;13:472-476.

5. Craven DE, Kunches LM, Lichtenberg DA, et al: Nosocomial infection and fatality in medical and surgical intensive care unit patients. Arch Intern Med 1988;148:1161-1168.

6. Nystrom B, Frederici $H$, von Euler $C$ : Bacterial colonization and infection in 
n intensive care unit. Intensive Care Med 1988; 14:34-38

7. Chandrasekar PH, Kruse JA, Mathews MF: Nosocomial infection among patients in different types of intensive care units at a city hospital. Crit Care Med 1986;14:508-510.

8. Schandorf WA, Brown RB, Sands M, Hosmer D: Infections in a coronary care unit. Am I Cardiol 1985;56: 757-759.

9. Miller RM, Polakavetz SH, Hornick RB, Cowley RA: Analysis of infections acquired by the severely injured patient. Surg Gynecol Obstet 1973;137:7-10

10. Marshall WG Jr, Dimick AR: The natural history of major burns with multiple subsystem failure. J Trauma 1983;23:102-105.

11. Pine RW, Wertz MJ, Lennard ES, et al: Determinants of organ malfunction or death in patients with intra-abdominal sepsis. A discriminant analysis. Arch Surg 1983;118:242-249.

12. Centers for Disease Control and Prevention. Monitoring hospitalacquired infections to promote patient safety-United States, 1990-1999. MMWR Morb Mortal Wkly Rep 2000;49:149-153.

13. Berenholtz SM, Pronovost PI, Lisett PA, et al: Eliminating catheter-related bloodstream infection in the intensive care unit. Crit Care Med 2004;32: 2014-2020.

14. Reduction in central line-associated bloodstream infections among patients in intensive care unitsPennsylvania, April 2001-March 2005. MMWR Morb Mortal Wkly Rep 2005;54:1013-1016.

15. Pronovost P, Needham D, Berenholtz $S$, et al: An intervention to decrease catheter-related bloodstream infections in the ICU. N Engl J Med 2006;355: 2725-2732.

16. Horan TC, Gaynes RP: Surveillance of nosocomial infections. In Mayhall CG (ed): Hospital Epidemiology and Infection Control, 3rd ed. Philadelphia, Lippincott Williams \& Wilkins, 2004, pp 1659-1702.

17. Crnich Cl, Maki DG: The role of intravascular devices in sepsis. Curr Infect Dis Rep 2001;3:497-506.

18. Wunderink RG, Mayhall CG, Gibert C: Methodology for clinical investigation of ventilator-associated pneumonia. Epidemiology and therapeutic intervention. Chest 1992;102(5 Suppl 1):580S-588S

19. Wenzel RP, Thompson RL, Landry SM, et al: Hospital-acquired infections in intensive care unit patients: An overview with emphasis on epidemics. Infect Control 1983;4:371-375.

20. Northey D, Adess ML, Hartsuck JM, Rhoades ER: Microbial surveillance in a surgical intensive care unit. Surg Gynecol Obstet 1974;139:321-325.

21. Caplan ES, Hoyt N: Infection surveillance and control in the severely traumatized patient. Am J Med 1981;70:638-640.

22. Daschner FD, Frey P, Wolff G, et al: Nosocomial infections in intensive care wards: A multicenter prospective study. Intensive Care Med 1982;8:5-9.

23. Goldmann DA, Durbin WA Jr, Freeman J: Nosocomial infections in a neonatal intensive care unit. I Infect Dis 1981;144:449-459.

24. Goldmann DA, Freeman J, Durbin WA Ir: Nosocomial infection and death in a neonatal intensive care unit. J Infect Dis 1983; 147:635-641.

25. Donowitz LG, Wenzel RP, Hoyt JW: High risk of hospital-acquired infection in the ICU patient. Crit Care Med 1982;10:355-357

26. Jarvis WR, Edwards JR, Culver $\mathrm{DH}$, et al: Nosocomial infection rates in adult and pediatric intensive care units in the United States. National Nosocomial Infections Surveillance System. Am J Med 1991;91: 185S-191S

27. Nosocomial infection rates for interhospital comparison: Limitations and possible solutions. A Report from the National Nosocomial Infections Surveillance (NNIS) System. Infect Control Hosp Epidemiol 1991;12: 609-621.

28. Maki DG: Control of colonization and transmission of pathogenic bacteria in the hospital. Ann Intern Med 1978;89(5 Pt 2 Suppl):777-780.

29. Wenzel RP, Osterman CA, Donowitz LG, et al: Identification of procedurerelated nosocomial infections in high-risk patients. Rev Infect Dis 1981;3:701-707.

30. Emori TG, Culver DH, Horan TC, et al: National nosocomial infections surveillance system (NNIS): Description of surveillance methods. Am J Infect Control 1991;19:19-35.

31. Joint Commission on Accreditation of Healthcare Organizations. Comprehensive Accreditation Manual for Hospitals. Oak Brook, III, JCAHO, 2007.

32. Maki DG: Nosocomial bacteremia. An epidemiologic overview. Am J Med 1981;70:719-732.

33. Richards MJ, Edwards JR, Culver DH, Gaynes RP: Nosocomial infections in combined medical-surgical intensive care units in the United States. Infect Control Hosp Epidemiol 2000;21: 510-515.

34. Kelly CP, Pothoulakis C, LaMont JT: Clostridium difficile colitis. $\mathrm{N}$ Engl J Med 1994:330:257-262

35. Richards MJ, Edwards JR, Culver DH Gaynes RP: Nosocomial infections in medical intensive care units in the United States. National Nosocomial Infections Surveillance System. Crit Care Med 1999;27:887-892.

36. Rhame FS, Streifel AJ, Kersey JH Jr, McGlave PB: Extrinsic risk factors for pneumonia in the patient at high risk of infection. Am J Med 1984;76:42-52.

37. Sherertz RI, Belani A, Kramer BS, et al: Impact of air filtration on nosocomial Aspergillus infections. Unique risk of bone marrow transplant recipients. Am J Med 1987;83:709-718.

38. Walsh TJ, Dixon DM: Nosocomial aspergillosis: Environmenta microbiology, hospital epidemiology, diagnosis and treatment. Eur I Epidemiol 1989;5:131-142.

39. Sinnott JTt, Gilchrist LS, Ellis L: Respiratory syncytial virus. Infect Control Hosp Epidemiol 1988;9: 465-468.

40. Cone R, Mohan K, Thouless M, Corey $\mathrm{L}$ : Nosocomial transmission of rotavirus infection. Pediatr Infect Dis J 1988;7:103-109.

41. Hart CA, Makin T: Legionella in hospitals: A review. J Hosp Infect 1991;18(Suppl A):481-489.

42. Banerjee SN, Emori TG, Culver DH et al: Secular trends in nosocomial primary bloodstream infections in the United States, 1980-1989. National Nosocomial Infections Surveillance System. Am J Med 1991;91:86S-89S.

43. Schaberg DR, Culver DH, Gaynes RP: Major trends in the microbial etiology of nosocomial infection. Am I Med 1991;91:72S-75S.

44. Centers for Disease Control and Prevention: Trends in antibiotic resistance in National Nosocomial Infections Surveillance (NNIS) system hospitals, 1989-2004. Available at http://www.cdc.gov/ncidod/dhqp/pdf/ ar/ICU_RESTrend1995-2004.pdf (Accessed January 15, 2007)

45. Weinstein RA: Nosocomial infection update. Emerg Infect Dis 1998;4: 416-420.

46. Ayala A, Perrin MM, Wagner MA, Chaudry $\mathrm{IH}$ : Enhanced susceptibility to sepsis after simple hemorrhage. Depression of Fc and C3b receptormediated phagocytosis. Arch Surg 1990;125:70-74; discussion 4-5.

47. Gardner ID: The effect of aging on susceptibility to infection. Rev Infect Dis 1980;2:801-810.

48. Gorse G], Messner RL, Stephens ND: Association of malnutrition with nosocomial infection. Infect Control Hosp Epidemiol 1989;10:194-203.

49. Fauci AS, Dale DC, Balow JE: Glucocorticosteroid therapy: Mechanisms of action and clinical considerations. Ann Intern Med 1976;84:304-315.

50. Santos GW, Owens AH Jr Sensenbrenner LL: Effects of selected cytotoxic agents on antibody production in man; a preliminary report. Ann N Y Acad Sci 1964;114: 404-423.

51. Rubin RH: Infection in the renal and liver transplant patient. In Rubin RH, Young LS (eds): Clinical Approach to Infection in the Compromised Host. New York, Plenum, 1988

52. Atherton ST, White DJ: Stomach as source of bacteria colonising respiratory tract during artificial ventilation. Lancet 1978;2:968-969.

53. Messori A, Trippoli S, Vaiani M, et al: Bleeding and pneumonia in intensive care patients given ranitidine and sucralfate for prevention of stress ulcer: Meta-analysis of randomised controlled trials. BMJ 2000;321: 1103-1106.

54. Agarwal N, Murphy JG, Cayten CG, Stahl WM: Blood transfusion increases the risk of infection after trauma. Arch Surg 1993;128:171-176; discussion 6-7.

55. Wenzel RP, Nettleman MD, Jones RN, Pfaller MA: Methicillin-resistant Staphylococcus aureus: Implications for the 1990s and effective control measures. Am J Med 1991;91:221S-227S.

56. Gray JW, Pedler SJ: Antibiotic-resistant enterococci. I Hosp Infect 1992;21: $1-14$. 
57. McGowan JE Jr: Antibiotic resistance in hospital bacteria: Current patterns, modes of appearance or spread, and economical impact. Rev Med Microbiol 1991;2:161-169.

58. Sanders CC, Sanders WE Jr: betaLactam resistance in gram-negative bacteria: global trends and clinical impact. Clin Infect Dis 1992:15:824-839.

59. Favero MS, Carson LA, Bond WW, Petersen NJ: Pseudomonas aeruginosa: Growth in distilled water from hospitals. Science 1971;173:836-838.

60. Wadowsky RM, Wolford R, McNamara AM, Yee RB: Effect of temperature, $\mathrm{pH}$, and oxygen level on the multiplication of naturally occurring Legionella pneumophila in potable water. Appl Environ Microbiol 1985;49:1197-1205.

61. Cross AS, Sadoff JC, Iglewski BH, Sokol PA: Evidence for the role of toxin $A$ in the pathogenesis of infection with Pseudomonas aeruginosa in humans. J Infect Dis 1980;142:538-546.

62. Tucker KD, Carrig PE, Wilkins TD: Toxin A of Clostridium difficile is a potent cytotoxin. J Clin Microbiol 1990;28:869-871.

63. Schlievert PM, Shands KN, Dan BB, et al: Identification and characterization of an exotoxin from Staphylococcus aureus associated with toxic-shock syndrome. J Infect Dis 1981;143: 509-516.

64. Tojo M, Yamashita N, Goldmann DA, Pier GB: Isolation and characterization of a capsular polysaccharide adhesin from Staphylococcus epidermidis. Infect Dis 1988;157:713-722.

65. Falcieri E, Vaudaux P, Huggler E, et al: Role of bacterial exopolymers and host factors on adherence and phagocytosis of Staphylococcus aureus in foreign body infection. J Infect Dis 1987; 155:524-531.

66. Vaudaux P, Lew D, Waldvogel FA: Host-dependent pathogenic factors in foreign body infection: A comparison between Staphylococcus epidermidis and S. aureus. Zentralbl Bakteriol Suppl 1987:16:189-193.

67. Gristina AG, Jennings RA, Naylor PT, et al: Comparative in vitro antibiotic resistance of surface-colonizing coagulase-negative staphylococci. Antimicrob Agents Chemother 1989:33:813-816.

68. von Eiff C, Becker K, Machka K, et al: Nasal carriage as a source of Staphylococcus aureus bacteremia. Study Group. N Engl J Med 2001;344:11-16.

69. Moore EP, Williams EW: Hospital transmission of multiply antibioticresistant Streptococcus pneumoniae. I Infect 1988;16:199-200.

70. Pearson ML, Jereb JA, Frieden TR, et al: Nosocomial transmission of multidrugresistant Mycobacterium tuberculosis. A risk to patients and health care workers. Ann Intern Med 1992;117: 191-196.

71. Griffith $D E$, Hardeman JL, Zhang $Y$, et al: Tuberculosis outbreak among healthcare workers in a community hospital. Am J Resp Crit Care Med 1995;152:808-811.

72. Frieden TR, Sherman LF, Maw KL, et al: A multi-institutional outbreak of highly drug-resistant tuberculosis:
Epidemiology and clinical outcomes. JAMA 1996;276:1229-1235.

73. Atkinson WL, Markowitz LE, Adams NC, Seastrom GR: Transmission of measles in medical settings-United States, 1985-1989. Am J Med 1991:91:320S-324S.

74. Poland GA, Nichol KL: Medical students as sources of rubella and measles outbreaks. Arch Intern Med 1990;150:44-46.

75. Oliveira EC, Lee B, Colice GL: Influenza in the intensive care unit. I Intensive Care Med 2003;18:80-91.

76. Hare R, Thomas CG: The transmission of Staphylococcus aureus. BMJ 1956;2:840-844.

77. Reybrouck G: Role of the hands in the spread of nosocomial infections. J Hosp Infect 1983;4:103-110.

78. Leclair JM, Freeman J, Sullivan BF, et al: Prevention of nosocomial respiratory syncytial virus infections through compliance with glove and gown isolation precautions. $\mathrm{N}$ Engl J Med 1987;317:329-334.

79. Raad, II, Sherertz RJ, Russell BA Reuman PD: Uncontrolled nosocomial rotavirus transmission during a community outbreak. Am J Infect Control 1990;18:24-28

80. McFarland LV, Mulligan ME, Kwok RY Stamm WE: Nosocomial acquisition of Clostridium difficile infection. $\mathrm{N}$ Engl J Med 1989:320:204-210.

81. Reagan DR, Pfaller MA, Hollis RJ, Wenzel RP: Characterization of the sequence of colonization and nosocomial candidemia using DNA fingerprinting and a DNA probe. I Clin Microbio 1990;28:2733-2738.

82. Dougherty SH: Pathobiology of infection in prosthetic devices. Rev Infect Dis 1988;10:1102-1117.

83. Nakashima AK, Allen JR, Martone WJ, et al: Epidemic bullous impetigo in a nursery due to a nasal carrier of Staphylococcus aureus: Role of epidemiology and control measures. Infect Control 1984;5:326-331.

84. Viglionese A, Nottebart VF, Bodman $H A$, Platt R: Recurrent group A streptococcal carriage in a health care worker associated with widely separated nosocomial outbreaks. Am J Med 1991;91:329S-333S.

85. Grieble HG, Bird TJ, Nidea HM, Miller CA: Chute-hydropulping waste disposal system: A reservoir of enteric bacilli and pseudomonas in a modern hospital. J Infect Dis 1974; 130:602-607.

86. Olson B, Weinstein RA, Nathan C, et al: Epidemiology of endemic Pseudomonas aeruginosa: Why infection control efforts have failed. I Infect Dis 1984;150:808-816.

87. Olson B, Weinstein RA, Nathan C, et al: Occult aminoglycoside resistance in Pseudomonas aeruginosa:

Epidemiology and implications for therapy and control. J Infect Dis 1985; 152:769-774.

88. Weinstein RA: Endemic emergence of cephalosporin-resistant Enterobacter: Relation to prior therapy. Infect Control 1986;7(2 Suppl):120-123.

89. Flynn DM, Weinstein RA, Nathan C, et al: Patients' endogenous flora as the source of "nosocomial" Enterobacter in cardiac surgery. I Infect Dis

1987; $156: 363-368$

90. Weinstein RA: Epidemiology and control of nosocomial infections in adult intensive care units. Am J Med 1991;91:179S-184S

91. Shooter RA, Gaya H, Cooke EM, et al: Food and medicaments as possible sources of hospital strains of Pseudomonas aeruginosa. Lancet 1969; 1:1227-1229.

92. Thurn I, Crossley K, Gerdts A, et al: Enteral hyperalimentation as a source of nosocomial infection. J Hosp Infect 1990;15:203-217.

93. Rutala WA, Kennedy VA, Loflin HB, Sarubbi FA Jr: Serratia marcescens nosocomial infections of the urinary tract associated with urine measuring containers and urinometers. Am J Med 1981;70:659-663.

94. Cobben NA, Drent M, Jonkers M, et al: Outbreak of severe Pseudomonas aeruginosa respiratory infections due to contaminated nebulizers. J Hosp Infect 1996;33:63-70.

95. Crnich Cl, Safdar N, Maki DG: The role of the intensive care unit environment in the pathogenesis and prevention of ventilator-associated pneumonia. Respir Care 2005;50:813836; discussion 36-8.

96. Mermel LA, Maki DG: Epidemic bloodstream infections from hemodynamic pressure monitoring: Signs of the times. Infect Control Hosp Epidemiol 1989;10:47-53.

97. Favero MS, Petersen NI, Boyer KM et al: Microbial contamination of renal dialysis systems and associated health risks. Trans Am Soc Artif Intern Organs 1974;20A:175-183.

98. Berkelman RL, Godley J, Weber JA, et al: Pseudomonas cepacia peritonitis associated with contamination of automatic peritoneal dialysis machines. Ann Intern Med 1982;96:456-458.

99. Alvarado Cl, Stolz SM, Maki DG: Nosocomial infections from contaminated endoscopes: A flawed automated endoscope washer. An investigation using molecular epidemiology. Am J Med 1991;91: 272S-280S.

100. Weber DI, Rutala WA: Lessons from outbreaks associated with bronchoscopy. Infect Control Hosp Epidemiol 2001:22:403-408.

101. Srinivasan A, Wolfenden LL, Song $X$, et al: An outbreak of Pseudomonas aeruginosa infections associated with flexible bronchoscopes. $\mathrm{N}$ Engl J Med 2003:348:221-227.

102. Platt R, Polk BF, Murdock B, Rosner B: Risk factors for nosocomial urinary tract infection. Am I Epidemiol 1986;124:977-985.

103. Garibaldi RA, Burke JP, Dickman ML, Smith CB: Factors predisposing to bacteriuria during indwelling urethral catheterization. N Engl ] Med 1974:291:215-219.

104. Celis R, Torres A, Gatell JM, et al: Nosocomial pneumonia. A multivariate analysis of risk and prognosis. Chest 1988;93:318-324

105. Craven DE, Kunches LM, Kilinsky V et al: Risk factors for pneumonia and fatality in patients receiving continuous mechanical ventilation. Am Rev Respir Dis 1986;133:792-796. 
106. Nichols RL: Surgical wound infection. Am I Med 1991;91:54S-64S.

107. Maki DG: Infections caused by intravascular devices used for infusion therapy: Pathogenesis, prevention and management. In Bisno AL, Waldvogel FA (eds): Infections Associated with Indwelling Medical Devices, 2nd ed. Washington, DC, ASM Press, 1994.

108. Mayhall CG, Archer NH, Lamb VA, et al: Ventriculostomy-related infections. A prospective epidemiologic study. N Engl I Med 1984:310:553-559.

109. Bleichner G, Thomas O, Sollet JP: Diarrhea in intensive care: diagnosis and treatment. Int J Antimicrob Agents 1993;3:33.

110. Wey SB, Mori M, Pfaller MA, et al: Risk factors for hospital-acquired candidemia. A matched case-control study. Arch Intern Med 1989;149:2349-2353.

111. Bross J, Talbot GH, Maislin G, et al: Risk factors for nosocomial candidemia: A case-control study in adults without leukemia. Am J Med 1989;87:614-620.

112. Fraser VJ, Jones $M$, Dunkel J, et al: Candidemia in a tertiary care hospital: Epidemiology, risk factors, and predictors of mortality. Clin Infect Dis 1992; 15:414-421.

113. Infection surveillance and control programs in U.S. hospitals: An assessment. MMWR Morb Mortal Wkly Rep 1978;27.

114. Haley RW, Quade D, Freeman HE, et al: Study on efficacy of nosocomial infection control (SENIC project): Summary of study design. Am Epidemiol 1980;111:472-485.

115. Haley RW, Culver DH, White JW, et al The efficacy of infection surveillance and control programs in preventing nosocomial infections in US hospitals. Am J Epidemiol 1985;121:182-205.

116. Haley RW: The development of infection surveillance and control programs. In Bennett JV, Brachman PS (eds): Hospital Infections, 4th ed. Philadelphia, Lippincott-Raven Publishers, 1998, pp 53-64.

117. Haley RW, Morgan WM, Culver DH, et al: Hospital infection control: Recent progress and opportunities under prospective payment. Am J Infect Control 1985; 13:97-108.

118. U.S. Department of Health and Human Services. Healthy People 2010. Washington, DC, U.S. Government Printing Office, 2000

119. Institute of Medicine. To Err is Human: Building a Safer Health System. Washington, DC, National Academy Press, 1999.

120. Haley RW, White JW, Culver DH, Hughes JM: The financial incentive for hospitals to prevent nosocomial infections under the prospective payment system. An empirical determination from a nationally representative sample. IAMA 1987;257:1611-1614.

121. Goldmann DA: Nosocomial infection control in the United States of America. J Hosp Infect 1986;8:116-128.

122. Haley RW: Managing Hospital Infection Control for CostEffectiveness. Chicago, American Hospital Association, 1986.
123. Wenzel RP, Pfaller MA: Infection control: the premier quality assessment program in United States hospitals. Am J Med 1991;91:27S-31S.

124. Scheckler WE: Interim report of the Quality Indicator Study Group. Infect Control Hosp Epidemiol 1994;15: 265-268.

125. Updated U.S. Public Health Service Guidelines for the Management of Occupational Exposures to HBV, HCV, and HIV and Recommendations for Postexposure Prophylaxis. MMWR Recomm Rep 2001;50(RR-11):1-52.

126. Jensen PA, Lambert LA, lademarco MF, Ridzon R: Guidelines for preventing the transmission of Mycobacterium tuberculosis in health-care settings, 2005. MMWR Recomm Rep 2005;54:1-141

127. Rutala WA, Mayhall CG: Medical waste. Infect Control Hosp Epidemiol 1992;13:38-48.

128. Rutala WA, Odette RL, Samsa GP: Management of infectious waste by US hospitals. JAMA 1989;262: 1635-1640.

129. O'Grady NP, Alexander M, Dellinger $E P$, et al: Guidelines for the prevention of intravascular catheter-related infections. Clin Infect Dis 2002;35 1281-1307.

130. Wong ES: Guideline for prevention of catheter-associated urinary tract infections. Am J Infect Control 1983;11:28-36

131. Tablan OC, Anderson LJ, Besser R, et al: Guidelines for preventing healthcare-associated pneumonia, 2003: Recommendations of CDC and the Healthcare Infection Control Practices Advisory Committee. MMWR Recomm Rep 2004;53(RR-3):1-36.

132. Jones RN: Global epidemiology of antimicrobial resistance among community-acquired and nosocomial pathogens: A five-year summary from the SENTRY Antimicrobial Surveillance Program (1997-2001). Semin Respir Crit Care Med 2003;24:121-134.

133. National Nosocomial Infections Surveillance (NNIS) System Report, data summary from January 1992 through June 2004, issued October 2004. Am J Infect Control 2004; 32:470-485

134. McGowan JE, Weinstein RA: The role of the laboratory in control of nosocomial infection. In Bennett JV, Brachman PS (eds): Hospital Infections, 4th ed. Philadelphia, Lippincott-Raven Publishers, 1998, pp 143-164.

135. Bouam S, Girou E, Brun-Buisson C, et al: An intranet-based automated system for the surveillance of nosocomial infections: Prospective validation compared with physicians' self-reports. Infect Control Hosp Epidemiol 2003;24:51-55.

136. Wright MO, Perencevich EN, Novak C et al: Preliminary assessment of an automated surveillance system for infection control. Infect Control Hosp Epidemiol 2004:25:325-332.

137. Ernst EJ, Diekema DJ, BootsMiller BJ, et al: Are United States hospitals following national guidelines for the analysis and presentation of cumulative antimicrobial susceptibility data? Diagn Microbiol Infect Dis 2004;49:141-145.
138. Lautenbach E, Nachamkin I: Analysis and presentation of cumulative antimicrobial susceptibility data (antibiograms): Substantial variability across medical centers in the United States. Infect Control Hosp Epidemiol 2006;27:409-412.

139. Zapantis A, Lacy MK, Horvat RT, et al: Nationwide antibiogram analysis using NCCLS M39-A guidelines. I Clin Microbiol 2005;43:2629-2634.

140. National Committee for Clinical Laboratory Standards (NCCLS). Analysis and presentation of cumulative antimicrobial susceptibility test data: Approved standard. NCCLS document M39-A. Wayne, Pa, NCCLS 2002.

141. Pestotnik SL, Classen DC, Evans RS, Burke JP: Implementing antibiotic practice guidelines through computer assisted decision support: Clinical and financial outcomes. Ann Intern Med 1996;124:884-890.

142. McGregor JC, Weekes E, Forrest GN, et al: Impact of a computerized clinical decision support system on reducing inappropriate antimicrobial use: $A$ randomized controlled trial. J Am Med Inform Assoc 2006;13:378-384.

143. Maki DG: Epidemic nosocomial bacteremias. In Wenzel RP (ed): Handbook of Hospital Infection. Boca Raton, Fla, CRC Press, 1981, pp 371-512.

144. Tenover FC: Rapid detection and identification of bacterial pathogens using novel molecular technologies: Infection control and beyond. Clin Infect Dis 2007;44:418-423.

145. Thorburn K, Kerr S, Taylor N, van Saene HK: RSV outbreak in a paediatric intensive care unit. I Hosp Infect 2004;57:194-201.

146. Quindos G: New microbiological techniques for the diagnosis of invasive mycoses caused by filamentous fungi. Clin Microbio Infect 2006;12(Suppl 7):40-52

147. Herwaldt LA, Pfaller MA, Weber S: Microbial molecular techniques. In Thomas IC, Weber DJ (eds): Epidemiologic Methods for the Study of Infectious Diseases. New York, Oxford University Press, 2001, pp 163-191.

148. Poutanen SM, Tompkins LS: Molecular methods in nosocomial epidemiology. In Wenzel RP (ed): Prevention and Control of Nosocomial Infections. Philadelphia, Lippincott Williams \& Wilkins, 2003 pp 481-499.

149. Schwartz DH, Laeyendecker OB, Arango-Jaramillo $S$, et al: Extensive evaluation of a seronegative participant in an HIV-1 vaccine trial as a result of false-positive PCR. Lancet 1997:350:256-259.

150. Lievano FA, Reynolds MA, Waring AL, et al: Issues associated with and recommendations for using PCR to detect outbreaks of pertussis. J Clin Microbiol 2002;40:2801-2805.

151. Boyce JM, Potter-Bynoe G, Chenevert C, King T: Environmental contamination due to methicillinresistant Staphylococcus aureus: Possible infection control implications. Infect Control Hosp Epidemiol 1997; 18:622-627. 
152. Bonten Ml, Hayden MK, Nathan C, et al: Epidemiology of colonisation of patients and environment with vancomycin-resistant enterococci. Lancet 1996;348:1615-1619.

153. Aygun G, Demirkiran O, Utku T, et al: Environmental contamination during a carbapenem-resistant Acinetobacter baumannii outbreak in an intensive care unit. I Hosp Infect 2002;52 259-262.

154. Maki DG, Alvarado CJ, Hassemer CA, Zilz MA: Relation of the inanimate hospital environment to endemic nosocomial infection. $N$ Engl J Med 1982;307:1562-1566.

155. McGowan JE Jr: Environmental factors in nosocomial infection: A selective focus. Rev Infect Dis 1981;3:760-769.

156. Boyce JM, White RL, Causey WA, Lockwood WR: Burn units as a source of methicillin-resistant Staphylococcus aureus infections. JAMA 1983;249: 2803-2807.

157. Samore $M H$, Venkataraman $L$, DeGirolami PC, et al: Clinical and molecular epidemiology of sporadic and clustered cases of nosocomial Clostridium difficile diarrhea. Am J Med 1996;100:32-40.

158. Duckro AN, Blom DW, Lyle EA, et al: Transfer of vancomycin-resistant enterococci via health care worker hands. Arch Intern Med 2005;165: 302-307.

159. Getchell-White SI, Donowitz LG, Groschel DH: The inanimate environment of an intensive care unit as a potential source of nosocomial bacteria: Evidence for long survival of Acinetobacter calcoaceticus. Infect Control Hosp Epidemiol 1989;10: 402-407.

160. Neely AN: A survey of gram-negative bacteria survival on hospital fabrics and plastics. I Burn Care Rehabil 2000;21:523-527.

161. Mayfield JL, Leet T, Miller J, Mundy LM: Environmental control to reduce transmission of Clostridium difficile. Clin Infect Dis 2000;31:995-1000.

162. Needleman I, Buerhaus $P$, Mattke $S$, et al: Nurse-staffing levels and the quality of care in hospitals. N Engl J Med 2002:346:1715-1722.

163. Hugonnet S, Harbarth S, Sax H, et al: Nursing resources: A major determinant of nosocomial infection? Curr Opin Infect Dis 2004;17:329-333.

164. Harbarth S, Sudre P, Dharan S, et al: Outbreak of Enterobacter cloacae related to understaffing, overcrowding, and poor hygiene practices. Infect Control Hosp Epidemiol 1999;20: 598-603.

165. Pittet D, Mourouga P, Perneger TV: Compliance with handwashing in a teaching hospital. Ann Intern Med 1999; 130:126-130.

166. Harvey MA: Critical-care-unit bedside design and furnishing: Impact on nosocomial infections. Infect Control Hosp Epidemiol 1998;19:597-601.

167. du Moulin G: Minimizing the potential for nosocomial pneumonia: Architectural, engineering, and environmental considerations for the intensive care unit. Eur J Clin Microbiol Infect Dis 1989;8:69-74.

168. Mulin B, Rouget $C$, Clement $C$, et al: Association of private isolation rooms with ventilator-associated Acinetobacter baumanii pneumonia in a surgical intensive-care unit. Infect Control Hosp Epidemiol 1997;18:499-503.

169. Shirani KZ, McManus AT, Vaughan $G M$, et al: Effects of environment on infection in burn patients. Arch Surg 1986;121:31-36.

170. Carter CD, Barr BA: Infection control issues in construction and renovation. Infect Control Hosp Epidemiol 1997; 18:587-596.

171. Boyce JM, Pittet D, Healthcare Infection Control Practices Advisory Committee: Society for Healthcare Epidemiology of America. Association for Professionals in Infection Control. Infectious Diseases Society of America. Hand Hygiene Task Force Guideline for Hand Hygiene in Health-Care Settings: Recommendations of the Healthcare nfection Control Practices Advisory Committee and the HICPAC/SHEA/ APIC/IDSA Hand Hygiene Task Force. Infect Control Hosp Epidemiol 2002;23(12 Suppl):S3-40.

172. Pittet D, Hugonnet $S$, Harbarth $S$, et al: Effectiveness of a hospital-wide programme to improve compliance with hand hygiene. Lancet 2000;356:1307-1312

173. Trautmann $M$, Michalsky $T$, Wiedeck $\mathrm{H}$, et al: Tap water colonization with Pseudomonas aeruginosa in a surgical intensive care unit (ICU) and relation to Pseudomonas infections of ICU patients. Infect Control Hosp Epidemiol 2001;22:49-52.

174. Garner JS, Healthcare Infection Contro Practices Advisory Committee: Guideline for isolation precautions in hospitals. Am J Infect Control 2007; (in press).

175. Anaissie EJ, Penzak SR, Dignani MC: The hospital water supply as a source of nosocomial infections: A plea for action. Arch Intern Med 2002;162:1483-1492.

176. Weber DI, Rutala WA, Blanchet CN, et al: Faucet aerators: A source of patient colonization with Stenotrophomonas maltophilia. Am I Infect Contro 1999;27:59-63.

177. Simor $A E$, Lee $M$, Vearncombe $M$, et al: An outbreak due to multiresistan Acinetobacter baumannii in a burn unit: Risk factors for acquisition and management. Infect Control Hosp Epidemiol 2002;23:261-267.

178. Wang $\mathrm{SH}$, Sheng $\mathrm{WH}$, Chang $\mathrm{YY}$, et al: Healthcare-associated outbreak due to pan-drug resistant Acinetobacter baumannii in a surgical intensive care unit. J Hosp Infect 2003;53:97-102.

179. Sabria M, Yu VL: Hospital-acquired legionellosis: Solutions for a preventable infection. Lancet Infect Dis 2002;2:368-373.

180. Haley CE, Cohen ML, Halter J, Meyer RD: Nosocomial Legionnaires' disease: A continuing common-source epidemic at Wadsworth Medica Center. Ann Intern Med 1979;90: 583-586.

181. Centers for Disease Control and Prevention: Guidelines for preventing health-care-associated pneumonia, 2003. Recommendations of the CDC and the Healthcare Infection Control Practices Advisory Committee. MMWR Morb Mortal Wkly Rep 2004;53(RR-3).
182. Benin AL, Benson RF, Besser RE: Trends in Legionnaires disease, 1980-1998: Declining mortality and new patterns of diagnosis. Clin Infect Dis 2002;35: 1039-1046.

183. Yu VL: Resolving the controversy on environmental cultures for Legionella: A modest proposal. Infect Control Hosp Epidemiol 1998; 19:893-897.

184. Kool JL, Fiore AE, Kioski CM, et al: More than 10 years of unrecognized nosocomial transmission of Legionnaires' disease among transplant patients. Infect Control Hosp Epidemiol 1998;19:898-904.

185. Lepine LA, Jernigan DB, Butler JC, et al: A recurrent outbreak of nosocomial Legionnaires' disease detected by urinary antigen testing: Evidence for long-term colonization of a hospital plumbing system. Infect Control Hosp Epidemiol 1998;19:905-910.

186. Alary M, Joly JR: Factors contributing to the contamination of hospital water distribution systems by legionellae. I Infect Dis 1992;165:565-569.

187. Kool JL, Bergmire-Sweat D, Butler JC, et al: Hospital characteristics associated with colonization of water systems by Legionella and risk of nosocomial legionnaires' disease: A cohort study of 15 hospitals. Infect Control Hosp Epidemiol 1999;20:798-805.

188. Fields BS, Benson RF, Besser RE: Legionella and Legionnaires' disease: 25 years of investigation. Clin Microbiol Rev 2002:15:506-526.

189. Helms CM, Massanari RM, Wenzel RP, et al: Legionnaires' disease associated with a hospital water system. A fiveyear progress report on continuous hyperchlorination. JAMA 1988;259: 2423-2427.

190. Muraca PW, Yu VL, Goetz A: Disinfection of water distribution systems for Legionella: A review of application procedures and methodologies. Infect Control Hosp Epidemiol 1990;11:79-88.

191. Farr BM, Gratz JC, Tartaglino JC, et al: Evaluation of ultraviolet light for disinfection of hospital water contaminated with Legionella. Lancet 1988:2:669-672.

192. Stout JE, Yu VL: Experiences of the first 16 hospitals using copper-silver ionization for Legionella control: Implications for the evaluation of other disinfection modalities. Infect Control Hosp Epidemiol 2003;24:563-568.

193. Rutala WA, Weber DJ: Disinfection and sterilization in health care facilities: What clinicians need to know. Clin Infect Dis 2004;39:702-709.

194. Rutala WA, Weber DJ: Guideline for disinfection and sterilization in healthcare facilities: Recommendations of the CDC Healthcare Infection Control Practices Advisory Committee. MMWR Morb Mortal Wkly Rep 2007; (in press).

195. Knight R: Creutzfeldt-Jakob disease: A rare cause of dementia in elderly persons. Clin Infect Dis 2006;43:340-346

196. Nelson DB, Jarvis WR, Rutala WA, et al: Multi-society guideline for reprocessing flexible gastrointestinal endoscopes. Society for Healthcare Epidemiology of America. Infect 
Control Hosp Epidemiol 2003;24:532-537.

197. Centers for Disease Control and Prevention: Contaminated povidoneiodine solution-Texas. MMWR Morb Mortal Wkly Rep 1989;38:133-134.

198. Panlilio AL, Beck-Sague CM, Siegel JD, et al: Infections and pseudoinfections due to povidone-iodine solution contaminated with Pseudomonas cepacia. Clin Infect Dis 1992;14: 1078-1083.

199. Alfa MJ, Sitter DL: In-hospital evaluation of orthophthalaldehyde as a high level disinfectant for flexible endoscopes. J Hosp Infect 1994;26: 15-26.

200. Mimoz O, Karim A, Mercat A, et al: Chlorhexidine compared with povidone-iodine as skin preparation before blood culture. A randomized, controlled trial. Ann Intern Med 1999;131:834-837.

201. Chaiyakunapruk N, Veenstra DL, Lipsky BA, Saint S: Chlorhexidine compared with povidone-iodine solution for vascular catheter-site care: A metaanalysis. Ann Intern Med 2002;136: 792-801.

202. Maki DG, Ringer M, Alvarado C): Prospective randomised trial of povidone-iodine, alcohol, and chlorhexidine for prevention of infection associated with central venous and arterial catheters. Lancet 1991;338:339-343.

203. Humar A, Ostromecki A, Direnfeld J, et al: Prospective randomized trial of $10 \%$ povidone-iodine versus $0.5 \%$ tincture of chlorhexidine as cutaneous antisepsis for prevention of central venous catheter infection. Clin Infect Dis 2000;31:1001-1007.

204. Maki DG, Zilz MA, Alvarado C): Evaluation of the antibacterial efficacy of four agents for handwashing. In Nelson JC, Grassi C (eds): Current chemotherapy and infectious disease: Proceedings of the 11th International Congress on Chemotherapy and the 19th Interscience Conference on Antimicrobial Agents and Chemotherapy; 1980. Washington, DC, American Society for Microbiology, 1980.

205. Horn WA, Larson EL, McGinley KJ, Leyden J): Microbial flora on the hands of health care personnel: Differences in composition and antibacterial resistance. Infect Control Hosp Epidemiol 1988;9:189-193.

206. Larson EL: Persistent carriage of gram negative bacteria on hands. Am I Infect Control 1981;9:112-119.

207. Graham M: Frequency and duration of handwashing in an intensive care unit. Am J Infect Control 1990; 18:77-81.

208. Larson E: A causal link between handwashing and risk of infection? Examination of the evidence. Infect Control 1988;9:28-36.

209. McCormick RD, Buchman TL, Maki DG: Double-blind, randomized trial of scheduled use of a novel barrier cream and an oil-containing lotion for protecting the hands of health care workers. Am J Infect Control 2000;28:302-310.

210. Pittet D, Boyce JM: Hand hygiene and patient care: Pursuing the
Semmelweiss legacy. Lancet Infect Dis 2001:0.9.

211. Maki DG, Hecht J: Antisepticcontaining handwashing agents reduce nosocomial infections: a prospective study. In Proceedings of the 22nd Interscience Conference on Antimicrobial Agents and Chemotherapy; 1982. Miami Beach, Fla, American Society for Microbiology, 1982.

212. Kaslow RA, Dixon RE, Martin SM, et al: Staphylococcal disease related to hospital nursery bathing practices. A nationwide epidemiologic investigation. Pediatrics 1973;51: 418-429.

213. Casewell M, Phillips I: Hands as route of transmission for Klebsiella species. BMJ 1977;2:1315-1317.

214. Bartzokas CA, Paton $\mathrm{JH}$, Gibson MF et al: Control and eradication of methicillin-resistant Staphylococcus aureus on a surgical unit. N Engl I Med 1984;311:1422-1425.

215. Onesko KM, Wienke EC: The analysis of the impact of a mild, low-iodine, lotion soap on the reduction of nosocomial methicillin-resistant Staphylococcus aureus: A new opportunity for surveillance by objectives. Infect Control 1987;8: 284-288.

216. Massanari RM: A crossover comparison of antiseptic soaps on nosocomial infection rates in intensive care units. Am J Infect Control 1984;12.

217. Doebbeling BN, Stanley GL, Sheetz $C T$, et al: Comparative efficacy of alternative hand-washing agents in reducing nosocomial infections in intensive care units. $\mathrm{N}$ Engl J Med 1992;327:88-93.

218. Rotter ML: Arguments for alcoholic hand disinfection. J Hosp Infect 2001;48(Suppl A):S4-8

219. Gordin FM, Schultz ME, Huber RA Gill JA: Reduction in nosocomial transmission of drug-resistant bacteria after introduction of an alcohol-based handrub. Infect Control Hosp Epidemiol 2005;26:650-653.

220. Lai KK, Fontecchio S, Melvin Z, Baker SP: Impact of alcohol-based, waterless hand antiseptic on the incidence of infection and colonization with methicillin-resistant Staphylococcus aureus and vancomycin-resistant enterococci. Infect Control Hosp Epidemiol 2006;27:1018-1024.

221. Larson EL, Eke PI, Laughon BE: Efficacy of alcohol-based hand rinses under frequent-use conditions. Antimicrob Agents Chemother 1986;30:542-544.

222. Larson EL, Aiello AE, Bastyr J, et al: Assessment of two hand hygiene regimens for intensive care unit personnel. Crit Care Med 2001;29: 944-951.

223. Centers for Disease Control and Prevention: Isolation techniques for use in hospitals. Washington, DC, U.S Government Printing Office, 1975.

224. Weinstein RA, Nathan C, Gruensfelder R, Kabins SA: Endemic aminoglycoside resistance in gram-negative bacilli: Epidemiology and mechanisms. J Infect Dis 1980;141:338-345.

225. Muto CA, Jernigan JA, Ostrowsky BE, et al: SHEA guideline for preventing nosocomial transmission of multidrug- resistant strains of Staphylococcus aureus and enterococcus. Infect Control Hosp Epidemio 2003;24:362-386.

226. Garner JS, Simmons BP: Guideline for isolation precautions in hospitals. Infect Control 1983:4(4 Suppl): 245-325.

227. Lynch $\mathrm{P}$, Cummings MJ, Roberts PL, et al: Implementing and evaluating a system of generic infection precautions: Body substance isolation. Am J Infect Control 1990;18:1-12.

228. Garner JS, Hughes JM: Options for isolation precautions. Ann Intern Med 1987; 107:248-250.

229. Karanfil LV, Murphy M, Josephson A, et al: A cluster of vancomycin-resistant Enterococcus faecium in an intensive care unit. Infect Control Hosp Epidemiol 1992;13:195-200.

230. Garner TK, Rimland D: Stethoscopes and infections. JAMA 1982;248:310.

231. Livornese LL Jr, Dias S, Samel C, et al: Hospital-acquired infection with vancomycin-resistant Enterococcus faecium transmitted by electronic thermometers. Ann Intern Med 1992;117:112-116.

232. Brooks SE, Veal RO, Kramer M, et al: Reduction in the incidence of Clostridium difficile-associated diarrhea in an acute care hospital and a skilled nursing facility following replacement of electronic thermometers with single-use disposables. Infect Control Hosp Epidemiol 1992;13:98-103.

233. Cepeda JA, Whitehouse T, Cooper B, et al: Isolation of patients in single rooms or cohorts to reduce spread of MRSA in intensive-care units: prospective two-centre study. Lancet 2005;365:295-304.

234. Agerton T, Valway S, Gore B, et al: Transmission of a highly drug-resistant strain (strain W1) of Mycobacterium tuberculosis. Community outbreak and nosocomial transmission via a contaminated bronchoscope. JAMA 1997;278:1073-1077.

235. Maloney SA, Pearson ML, Gordon MT et al: Efficacy of control measures in preventing nosocomial transmission of multidrug-resistant tuberculosis to patients and health care workers. Ann Intern Med 1995;122:90-95.

236. Recommendations for prevention of HIV transmission in health-care settings. MMWR Morb Mortal Wkly Rep 1987;36(Suppl 2):1S-18S.

237. Update: Universal precautions for prevention of transmission of human immunodeficiency virus, hepatitis B virus, and other bloodborne pathogens in health-care settings. MMWR Morb Mortal Wkly Rep 1988;37:377-382, 87-88.

238. Edmond M: Isolation. Infect Control Hosp Epidemiol 1997;18:58-64.

239. Occupational exposure to bloodborne pathogens-OSHA. Final rule. Fed Regist 1991;56:64004-64182.

240. Burke JF, Quinby WC, Bondoc CC, et al: The contribution of a bacterially isolated environment to the prevention of infection in seriously burned patients. Ann Surg 1977 186:377-387.

241. Johnson S, Gerding DN, Olson MM, et al: Prospective, controlled study of vinyl glove use to interrupt Clostridium 
difficile nosocomial transmission. Am Med 1990;88:137-140.

242. Maki DG: Through a glass darkly. Nosocomial pseudoepidemics and pseudobacteremias. Arch Intern Med 1980;140:26-28.

243. Doebbeling BN, Pfaller MA, Houston AK, Wenzel RP: Removal of nosocomial pathogens from the contaminated glove. Implications for glove reuse and handwashing. Ann Intern Med 1988;109:394-398.

244. Maki DG, McCormick RD, Zilz MA et al: A MRSA outbreak in an SICU during universal precautions: New epidemiology for nosocomial MRSA Downside for universal precautions. In Proceedings and Abstracts of the 30th Interscience Conference on Antimicrobial Agents and Chemotherapy; 1990, Atlanta, American Society for Microbiology, 1990.

245. Bubak ME, Reed CE, Fransway AF, et al: Allergic reactions to latex among health-care workers. Mayo Clin Proc 1992:67:1075-1079.

246. Kennedy PB, Gwaltney JMJ: Brief report: The detection of blood on gloved hands of central sterile supply personnel and cleaned instruments used for procedures on patient units. Infect Control Hosp Epidemiol 1988;9:117-118.

247. Neu HC: The crisis in antibiotic resistance. Science 1992;257 1064-1073.

248. Kunin CM: Resistance to antimicrobial drugs - a worldwide calamity. Ann Intern Med 1993;118:557-561.

249. Jarvis WR: Nosocomial outbreaks: the Centers for Disease Control's Hospital Infections Program experience, 1980 1990. Epidemiology Branch, Hospital Infections Program. Am J Med 1991;91:101S-106S.

250. Martone WJ, Jarvis WR, Culver $\mathrm{DH}$ et al: Incidence and nature of endemic and epidemic nosocomial infections. In Bennett JE, Brachman PS (eds): Hospital Infections, 3rd ed. Boston, Little, Brown, 1992.

251. Davies J: Inactivation of antibiotics and the dissemination of resistance genes. Science 1994:264:375-382.

252. Marr JJ, Moffet $\mathrm{HL}$, Kunin CM: Guidelines for improving the use of antimicrobial agents in hospitals: A statement by the Infectious Diseases Society of America. I Infect Dis 1988; 157:869-876.

253. Dellit TH, Owens RC, McGowan JE Jr, et al: Infectious Diseases Society of America and the Society for Healthcare Epidemiology of America guidelines for developing an institutional program to enhance antimicrobial stewardship. Clin Infect Dis 2007:44:159-177.

254. Avorn J, Harvey K, Soumerai SB, et al: Information and education as determinants of antibiotic use: Report of Task Force 5. Rev Infect Dis 1987;9 S286-296.

255. Collier J, Foster J: Management of a restricted drugs policy in hospital: The first five years' experience. Lancet 1986;2:331

256. Bartlett JC: Pocketbook of Infectious Disease Therapy. Baltimore, Williams \& Wilkins, 1993.
257. Sanford JP, Gilbert DN: Guide to Antimicrobial Therapy. Dallas, Antimicrobial Therapy, 1994.

258. Mangram AJ, Horan TC, Pearson ML, et al: Guideline for Prevention of Surgical Site Infection, 1999. Centers for Disease Control and Prevention (CDC) Hospital Infection Control Practices Advisory Committee. Am J Infect Control 1999;27:97-132.

259. DiPiro JT, Cheung RP, Bowden TA Jr, Mansberger JA: Single dose systemic antibiotic prophylaxis of surgical wound infections. Am J Surg 1986;152:552-559.

260. Maki DG, Tambyah PA: Engineering out the risk for infection with urinary catheters. Emerg Infect Dis 2001;7:342-347.

261. National Kidney Foundation: III. NKFK/DOQI Clinical Practice Guidelines for Vascular Access: Update 2000. Am J Kidney Dis 2001;37(1 Suppl 1): S137-181.

262. Maki D, Mermel L: Infections due to infusion therapy. In Bennett JV Brachman PS (eds): Hospital Infections, 4th ed. Philadelphia, Lippincott-Raven, 1998, pp 689-724.

263. Mermel LA: Prevention of intravascula catheter-related infections. Ann Intern Med 2000;132:391-402.

264. Pittet D, Tarara D, Wenzel RP. Nosocomial bloodstream infection in critically ill patients. Excess length of stay, extra costs, and attributable mortality. JAMA 1994;271:1598-1601.

265. Digiovine B, Chenoweth C, Watts C, Higgins $M$ : The attributable mortality and costs of primary nosocomial bloodstream infections in the intensive care unit. Am J Respir Crit Care Med 1999;160:976-981.

266. Soufir L, Timsit JF, Mahe C, et al: Attributable morbidity and mortality of catheter-related septicemia in critically ill patients: A matched, risk-adjusted, cohort study. Infect Control Hosp Epidemiol 1999;20:396-401.

267. Rello J, Ochagavia A, Sabanes E, et al: Evaluation of outcome of intravenous catheter-related infections in critically ill patients. Am J Respir Crit Care Med 2000;162:1027-1030.

268. Maki DG, Stolz SM, Wheeler $S$, Mermel LA: Prevention of central venous catheter-related bloodstream infection by use of an antisepticimpregnated catheter. A randomized, controlled trial. Ann Intern Med $1997 \cdot 127 \cdot 257-266$

269. Raad I, Darouiche R, Dupuis J, et al: Central venous catheters coated with minocycline and rifampin for the prevention of catheter-related colonization and bloodstream infections. A randomized, double-blind trial. Ann Intern Med 1997;127: 267-274.

270. O'Grady NP, Barie PS, Bartlett J, et al: Practice parameters for evaluating new fever in critically ill adult patients. Crit Care Med 1998;26:392-408.

271. Mermel LA, Farr BM, Sherertz RJ, et al: Guidelines for the management of intravascular catheter-related infections. Clin Infect Dis 2001 32:1249-1272

272. Safdar N, Maki DG: Inflammation at the insertion site is not predictive of catheter-related bloodstream infection with short-term, noncuffed central venous catheters. Crit Care Med 2002; 30:2632-2635

273. Mermel LA, Maki DG: Detection of bacteremia in adults: Consequences of culturing an inadequate volume of blood. Ann Intern Med 1993;119:270-272.

274. Dobbins BM, Kite $P$, Wilcox $M H$ : Diagnosis of central venous catheter related sepsis - a critical look inside. I Clin Pathol 1999;52:165-172.

275. Weinstein MP, Murphy JR, Reller LB, Lichtenstein KA: The clinical significance of positive blood cultures: A comprehensive analysis of 500 episodes of bacteremia and fungemia in adults. II. Clinical observations, with special reference to factors influencing prognosis. Rev Infect Dis 1983;5: 54-70.

276. Gaur AH, Giannini MA, Flynn PM, et al: Optimizing blood culture practices in pediatric immunocompromised patients: Evaluation of media types and blood culture volume. Pediatr Infect Dis | 2003:22:545-552.

277. DesJardin J, Falagas M, Ruthazer R, et al: Clinical utility of blood cultures drawn from indwelling central venous catheters in hospitalized patients with cancer. Ann Intern Med 1999;131: 641-647

278. Norberg A, Christopher NC, Ramundo $\mathrm{ML}$, et al: Contamination rates of blood cultures obtained by dedicated phlebotomy vs intravenous catheter. JAMA 2003;289:726-729.

279. Robinson JL: Sensitivity of a blood culture drawn through a single lumen of a multilumen, long-term, indwelling, central venous catheter in pediatric oncology patients. J Pediatr Hematol Onc 2002:24:72-74

280. Bates DW, Goldman L, Lee TH: Contaminant blood cultures and resource utilization. The true consequences of false-positive results. JAMA 1991:265:365-369.

281. Little JR, Murray PR, Traynor PS, Spitznagel E: A randomized trial of povidone-iodine compared with iodine tincture for venipuncture site disinfection: Effects on rates of blood culture contamination. Am I Med 1999;107:119-125.

282. Strand $C L$, Wajsbort RR, Sturmann $K$ Effect of iodophor vs iodine tincture skin preparation on blood culture contamination rate. JAMA 1993;269: 1004-1006.

283. Herwaldt LA, Geiss M, Kao C, Pfaller MA: The positive predictive value of isolating coagulase-negative staphylococci from blood cultures. Clin Infect Dis 1996:22:14-20.

284. Finkelstein R, Fusman R, Oren I, et al: Clinical and epidemiologic significance of coagulase-negative staphylococci bacteremia in a tertiary care university Israeli hospital. Am I Infect Control 2002;30:21-25.

285. Maki DG, Jarrett F, Sarafin HW: A semiquantitative culture method for identification of catheter-related infection in the burn patient. J Surg Res 1977:22:513-520.

286. Raad, II, Sabbagh MF, Rand KH, Sherertz RJ: Quantitative tip culture methods and the diagnosis of central venous catheter-related infections. 
Diagn Microbiol Infect Dis 1992;15:13-20.

287. Siegman-Igra $Y$, Anglim AM, Shapiro $D E$, et al: Diagnosis of vascular catheter-related bloodstream infection: A meta-analysis. J Clin Microbiol 1997:35:928-936.

288. Kristinsson KG, Burnett IA, Spencer RC: Evaluation of three methods for culturing long intravascular catheters. J Hosp Infect 1989;14:183-191.

289. Safdar N, Fine JP, Maki DG: Metaanalysis: methods for diagnosing intravascular device-related bloodstream infection. Ann Intern Med 2005; 142:451-466.

290. Collignon P, Chan R, Munro R: Rapid diagnosis of intravascular catheterrelated sepsis. Arch Intern Med 1987;147:1609-1612.

291. Darouiche RO, Raad II, Heard SO, et al: A comparison of two antimicrobialimpregnated central venous catheters. $\mathrm{N}$ Engl | Med 1999;340:1-8.

292. Ryan JJ, Abel R, Abbott W, et al: Catheter complications in total parenteral nutrition. A prospective study of 200 consecutive patients. N Engl I Med 1974;290:757-761.

293. Sitzmann JV, Townsend TR, Siler MC, Bartlett JG: Septic and technical complications of central venous catheterization. A prospective study of 200 consecutive patients. Ann Surg 1985:202:766-770.

294. Tacconelli E, Tumbarello M, Pittiruti M, et al: Central venous catheter-related sepsis in a cohort of 366 hospitalised patients. Eur J Clin Microbiol Infect Dis 1997;16:203-209.

295. Gowardman JR, Montgomery C Thirlwell $S$, et al: Central venous catheter-related bloodstream infections: An analysis of incidence and risk factors in a cohort of 400 patients. Intensive Care Med 1998;24:1034-1039.

296. Blot F, Nitenberg G, Chachaty E, et al: Diagnosis of catheter-related bacteraemia: A prospective comparison of the time to positivity of hub-blood versus peripheral-blood cultures. Lancet 1999;354:1071-1077.

297. Rijnders B], Verwaest C, Peetermans

$W E$, et al: Difference in time to positivity of hub-blood versus nonhubblood cultures is not useful for the diagnosis of catheter-related bloodstream infection in critically ill patients. Crit Care Med 2001;29: 1399-1403.

298. Maki DG, Kluger DM, Crnich C): The risk of bloodstream infection in adults with different intravascular devices: A systematic review of 200 published prospective studies. Mayo Clin Proc 2006;81:1159-1171.

299. Safdar N, Maki DG: The risk of catheter-related bloodstream infection with peripherally-inserted central venous catheters used in inpatients [abstract \#K-1435]. In Abstracts and Proceedings from the 41 st International Conference of Antimicrobial Agents and Chemotherapy. [abstract \#K-1435]: Washington, DC, American Society of Microbiology, 2001, p 428.

300. Safdar N, Maki DG: The incidence and pathogenesis of catheter-related bloodstream infection with arterial catheters [abstract \#K-81]. In Abstracts and Proceedings from the 42 nd Interscience Conference on Antimicrobial Agents and Chemotherapy. Washington, DC, American Society of Microbiology, 2002, p 299

301. Crnich CJ, Maki DG: The promise of novel technology for the prevention of intravascular device-related bloodstream infection. I. Pathogenesis and short-term devices. Clin Infect Dis 2002;34:1232-1242.

302. Maki DG, Cobb L, Garman JK, et al: An attachable silver-impregnated cuff for prevention of infection with central venous catheters: A prospective randomized multicenter trial. Am Med 1988;85:307-314

303. Mermel LA, McCormick RD, Springman SR, Maki DG: The pathogenesis and epidemiology of catheter-related infection with pulmonary artery Swan-Ganz catheters: A prospective study utilizing molecular subtyping. Am J Med 1991:91:197S-205S.

304. Linares J, Sitges-Serra A, Garau J, et al: Pathogenesis of catheter sepsis: A prospective study with quantitative and semiquantitative cultures of catheter hub and segments. J Clin Microbiol 1985;21:357-360.

305. Raad I, Costerton W, Sabharwal U, et al: Ultrastructural analysis of indwelling vascular catheters: A quantitative relationship between luminal colonization and duration of placement. J Infect Dis 1993;168: 400-407.

306. Maki D, Ringer M: Prospective study of arterial catheter-related infection: Incidence, sources of infection and risk factors [abstract]. In Abstracts and Proceedings from the 29th Interscience Conference on Antimicrobial Agents and Chemotherapy. Washington, DC, American Society of Microbiology 1989, p 1075

307. Maki DG, Klein BS, McCormick RD, et al: Nosocomial Pseudomonas pickettii bacteremias traced to narcotic tampering. A case for selective drug screening of health care personnel. JAMA 1991;265:981-986.

308. Maki D: The epidemiology and prevention of nosocomial bloodstream infections [abstract]. In Programs and Abstracts of the Third International Conference on Nosocomial Infections. Washington, DC, American Society of Microbiology, 1990, p 3.

309. Safdar N, Kluger DM, Maki DG: A review of risk factors for catheterrelated bloodstream infection caused by percutaneously inserted, noncuffed central venous catheters: Implications for preventive strategies. Medicine 2002;81:466-479.

310. Crnich Cl, Maki DG: Infections caused by intravascular devices: Epidemiology, pathogenesis, diagnosis, prevention, and treatment. In APIC Text of Infection Control and Epidemiology, 2nd ed. Washington, DC, Association for Professionals in Infection Control and Epidemiology, 2005, p 24.1-6.

311. Merrer J, De Jonghe B, Golliot F, et al: Complications of femoral and subclavian venous catheterization in critically ill patients: A randomized controlled trial. JAMA 2001;286 700-707.

312. Robert J, Fridkin SK, Blumberg HM, et al: The influence of the composition of the nursing staff on primary bloodstream infection rates in a surgical intensive care unit. Infect Control Hosp Epidemiol 2000;21: 12-17.

313. Maki DG, Kluger DM, Crnich CJ: The microbiology of intravascular devicerelated (IVDR) infection in adults: An analysis of 159 prospective studies and implications for prevention and treatment [abstract]. In Abstracts and Proceedings from the 40th Annual Meeting of the Infectious Disease Society of America. Chicago, Infectious Disease Society of America, 2002.

314. Dezfulian C, Lavelle I, Nallamothu BK, et al: Rates of infection for singlelumen versus multilumen central venous catheters: A meta-analysis. Crit Care Med 2003;31:2385-2390.

315. Goetz AM, Wagener MM, Miller JM, Muder RR: Risk of infection due to central venous catheters: Effect of site of placement and catheter type. Infect Control Hosp Epidemiol 1998; 19:842-845.

316. Moss $A H$, Vasilakis $C$, Holley JL, et al: Use of a silicone dual-lumen catheter with a Dacron cuff as a long-term vascular access for hemodialysis patients. Am J Kidney Dis 1990;16: 211-215.

317. Oliver MJ, Callery SM, Thorpe KE, et al: Risk of bacteremia from temporary hemodialysis catheters by site of insertion and duration of use: A prospective study. Kidney Int 2000;58:2543-2545.

318. Cimochowski GE, Worley E, Rutherford $W E$, et al: Superiority of the internal jugular over the subclavian access for temporary dialysis. Nephron 1990;54:154-161.

319. Schillinger F, Schillinger D, Montagnac $\mathrm{R}$, Milcent T: Post catheterisation vein stenosis in haemodialysis: Comparative angiographic study of 50 subclavian and 50 internal jugular accesses. Nephrol Dial Transplant 1991;6: 722-724

320. Raad, II, Hohn DC, Gilbreath BJ, et al: Prevention of central venous catheterrelated infections by using maximal sterile barrier precautions during insertion. Infect Control Hosp Epidemiol 1994;15:231-238.

321. Rijnders BJ, Van Wijngaerden E, Wilmer A Peetermans WE: Use of full sterile barrier precautions during insertion of arterial catheters: A randomized trial. Clin Infect Dis 2003:36:743-748.

322. Tomford JW, Hershey CO, McLaren CE, et al: Intravenous therapy team and peripheral venous catheter-associated complications. A prospective controlled study. Arch Intern Med 1984:144:1191-1194

323. Soifer NE, Borzak S, Edlin BR, Weinstein RA: Prevention of peripheral venous catheter complications with an intravenous therapy team: A randomized controlled trial. Arch Intern Med 1998;158:473-477

324. Sherertz RJ, Ely EW, Westbrook DM, et al: Education of physicians-intraining can decrease the risk for 
vascular catheter infection. Ann Intern Med 2000;132:641-648.

325. Eggimann $P$, Harbarth S, Constantin $\mathrm{M}$, et al: Impact of a prevention strategy targeted at vascular-access care on incidence of infections acquired in intensive care. Lancet 2000;355:1864-1868.

326. Maki DG, Knasinski V, Narans LL, Gordon B]: A randomized trial of a novel $1 \%$ chlorhexidine- $75 \%$ alcohol tincture versus $10 \%$ povidone-iodine for cutaneous disinfection with vascular catheters [abstract \#142]. In Abstracts and Proceedings from the 31st Annual Society for Healthcare Epidemiology of America Meeting. Toronto, Society for Healthcare Epidemiology of America, 2001, p 70.

327. Maki D, Will L: Colonization and infection associated with transparent dressings for central venous, arterial, and Hickman catheters: A comparative trial [abstract \#1241]. In Abstracts and Proceedings from the 24th Interscience Conference on Antimicrobial Agents and Chemotherapy. Washington, DC, American Society of Microbiology, 1984, p 991.

328. Maki DG, Stolz SS, Wheeler S, Merme LA: A prospective, randomized trial of gauze and two polyurethane dressings for site care of pulmonary artery catheters: Implications for catheter management. Crit Care Med 1994:22:1729-1737.

329. Maki D, Mermel LA, Martin M, et al: A highly semipermeable polyurethane dressing does not increase the risk of CVC-related BSI: A prospective, multicenter, investigator-blinded trial [abstract J-64]. In Abstracts and Proceedings from the 36th Interscience Conference on Antimicrobial Agents and Chemotherapy. Washington, DC, American Society for Microbiology, 1996, p 230.

330. Ricard P, Martin R, Marcoux JA: Protection on indwelling vascular catheters: Incidence of bacterial contamination and catheter-related sepsis. Crit Care Med 1985;13: 541-543.

331. Maki DG, Band JD: A comparative study of polyantibiotic and iodophor ointments in prevention of vascular catheter-related infection. Am J Med 1981;70:739-744.

332. Flowers RHd, Schwenzer KJ, Kopel RF, et al: Efficacy of an attachable subcutaneous cuff for the prevention of intravascular catheter-related infection. A randomized, controlled trial. JAMA 1989;261:878-883.

333. Hill RL, Fisher AP, Ware RJ, et al: Mupirocin for the reduction of colonization of internal jugular cannulae - a randomized controlled trial. J Hosp Infect 1990;15:311-321.

334. Sesso R, Barbosa D, Leme IL, et al: Staphylococcus aureus prophylaxis in hemodialysis patients using central venous catheter: Effect of mupirocin ointment. J Am Soc Nephrol 1998:9:1085-1092.

335. Miller MA, Dascal A, Portnoy J, Mendelson J: Development of mupirocin resistance among methicillin-resistant Staphylococcus aureus after widespread use of nasa mupirocin ointment. Infect Control Hosp Epidemiol 1996;17:811-813.

336. Zakrzewska-Bode A, Muytjens HL Liem KD, Hoogkamp-Korstanje JA: Mupirocin resistance in coagulasenegative staphylococci, after topical prophylaxis for the reduction of colonization of central venous catheters. I Hosp Infect 1995;31: 189-193.

337. Perez-Fontan $\mathrm{M}$, Rosales $\mathrm{M}$, RodriguezCarmona A, et al: Mupirocin resistance after long-term use for Staphylococcus aureus colonization in patients undergoing chronic peritoneal dialysis. Am J Kidney Dis 2002;39:337-341.

338. Prager RL, Silva J: Colonization of central venous catheters. South Med J 1984;77:458-461

339. Maki D, Will L: Study of polyantibiotic and povidone-iodine ointments on central venous and arterial catheter sites dressed with gauze or polyurethane dressing [abstract]. In Abstracts and Proceedings from the 26th Interscience Conference on Antimicrobial Agents and Chemotherapy. Washington, DC American Society of Microbiology, 1986, p 1041

340. Levin A, Mason Al, Jindal KK, et al: Prevention of hemodialysis subclavian vein catheter infections by topical povidone-iodine. Kidney Int 1991;40: 934-938.

341. Lai KK: Safety of prolonging peripheral cannula and i.v. tubing use from 72 hours to 96 hours. Am J Infect Control 1998;26:66-70.

342. Bregenzer T, Conen D, Sakmann P, Widmer AF: Is routine replacement of peripheral intravenous catheters necessary? Arch Intern Med 1998;158: 151-156

343. Pujol M, Hornero A, Saballs $M$, et al: Clinical epidemiology of bacteremia due to peripheral vascular catheter infections. In 43rd International Conference on Antimicrobial Agents and Chemotherapy [abstract K-2040]; 2003. Washington, DC, American Society of Microbiology, 2003.

344. Cobb DK, High KP, Sawyer RG, et al: A controlled trial of scheduled replacement of central venous and pulmonary-artery catheters. N Engl J Med 1992:327:1062-1068.

345. Cook D, Randolph A, Kernerman P, et al: Central venous cathete replacement strategies: A systematic review of the literature. Crit Care Med 1997:25:1417-1424.

346. Robinson D, Suhocki P, Schwab S]: Treatment of infected tunneled venous access hemodialysis catheters with guidewire exchange. Kidney Int 1998;53:1792-1794.

347. Beathard GA: Management of bacteremia associated with tunneledcuffed hemodialysis catheters. J Am Soc Nephrol 1999:10:1045-1049.

348. Maki DG, Hassemer CA: Endemic rate of fluid contamination and related septicemia in arterial pressure monitoring. Am J Med 1981;70:733-738.

349. Maki DG, Goldman DA, Rhame FS Infection control in intravenous therapy. Ann Intern Med 1973;79:867-887.
350. Maki DG, Botticelli JT, LeRoy ML, Thielke TS: Prospective study of replacing administration sets for intravenous therapy at 48- vs 72-hour intervals. 72 hours is safe and costeffective. JAMA 1987;258:1777-1781.

351. Luskin RL, Weinstein RA, Nathan C, et al: Extended use of disposable pressure transducers: A bacteriologic evaluation. JAMA 1986;255:916-920.

352. Shinozaki T, Deane RS, Mazuzan JE Jr, et al: Bacterial contamination of arterial lines. A prospective study. JAMA 1983;249:223-225.

353. Raad, II, Luna M, Khalil SA, et al: The relationship between the thrombotic and infectious complications of central venous catheters. JAMA 1994:271:1014-1016.

354. Mehall JR, Saltzman DA, Jackson RJ, Smith SD: Fibrin sheath enhances central venous catheter infection. Crit Care Med 2002;30:908-912.

355. Boraks P, Seale J, Price I, et al: Prevention of central venous catheter associated thrombosis using minidose warfarin in patients with haematological malignancies. $\mathrm{Br}$ J Haematol 1998:101:483-486.

356. Bern MM, Lokich JJ, Wallach SR, et al: Very low doses of warfarin can prevent thrombosis in central venous catheters. A randomized prospective trial. Ann Intern Med 1990;112: 423-428.

357. Randolph AG, Cook DJ, Gonzales CA, Andrew M: Benefit of heparin in peripheral venous and arterial catheters: Systematic review and metaanalysis of randomised controlled trials. BMJ 1998:316:969-975.

358. Mermel LA, Maki DG: Infectious complications of Swan-Ganz pulmonary artery catheters. Pathogenesis, epidemiology, prevention, and management. Am J Respir Crit Care Med 1994;149: 1020-1036.

359. Mermel LA Stolz SM, Maki DG: Surface antimicrobial activity of heparin-bonded and antisepticimpregnated vascular catheters. J Infect Dis 1993;167:920-924.

360. Crnich Cl, Maki DG: The promise of novel technology for the prevention of intravascular device-related bloodstream infection. II. Long-term devices. Clin Infect Dis 2002;34: 1362-1368.

361. Schears G): Summary of product trials for 10,164 patients: Comparing an intravenous stabilizing device to tape. I Infus Nurs 2006:29:225-231.

362. Yamamoto AJ, Solomon JA, Soulen $M C$, et al: Sutureless securement device reduces complications of peripherally inserted central venous catheters. J Vasc Interv Radiol 2002;13:77-81.

363. Cooper GL, Schiller AL, Hopkins CC: Possible role of capillary action in pathogenesis of experimental catheterassociated dermal tunnel infections. I Clin Microbiol 1988;26:8-12.

364. Maki DG, Mermel LA, Kluger DM, et al: The efficacy of a chlorhexidineimpregnated sponge (biopatch) for the prevention of intravascular catheter-related infection-a prospective, randomized, controlled, multicenter trial [abstract \#1430]. In 
Abstracts and Proceedings from the 40th Interscience Conference on Antimicrobial Agents and Chemotherapy. Washington, DC, American Society for Microbiology, 2000, p 422.

365. Garland JS, Alex CP, Mueller CD, et al: $A$ randomized trial comparing povidone-iodine to a chlorhexidine gluconate-impregnated dressing for prevention of central venous catheter infections in neonates. Pediatrics 2001:107:1431-1436.

366. Crnich CJ, Maki DG: Are antimicrobialimpregnated catheters effective? When does repetition reach the point of exhaustion? Clin Infect Dis 2005;41: 681-685.

367. Crnich CJ, Maki DG: Are antimicrobialimpregnated catheters effective? Don't throw out the baby with the bathwater. Clin Infect Dis 2004;38: 1287-1292.

368. Veenstra DL, Saint S, Saha S, et al: Efficacy of antiseptic-impregnated central venous catheters in preventing catheter-related bloodstream infection: A meta-analysis. JAMA 1999;281: 261-267.

369. Marin MG, Lee JC, Skurnick JH: Prevention of nosocomial bloodstream infections: Effectiveness of antimicrobial-impregnated and heparin-bonded central venous catheters. Crit Care Med 2000;28: 3332-3338.

370. Veenstra DL, Saint S, Sullivan SD: Cost-effectiveness of antisepticimpregnated central venous catheters for the prevention of catheter-related bloodstream infection. IAMA 1999;282:554-560.

371. Shorr AF, Humphreys CW, Helman DL: New choices for central venous catheters. Chest 2003;124:275-284

372. Saint S: Prevention of intravascula catheter-associated infections. In Shojania KG, Duncan BW, McDonald KM, Wachter RM (eds): Making Health Care Safer: A Critical Analysis of Patient Safety Practices. Rockville, Md, Agency for Healthcare Research and Quality, 2001, pp 163-184.

373. Henrickson KJ, Axtell RA, Hoover SM, et al: Prevention of central venous catheter-related infections and thrombotic events in immunocompromised children by the use of vancomycin/ciprofloxacin/ heparin flush solution: A randomized multicenter, double-blind trial. I Clin Oncol 2000;18:1269-1278.

374. Garland IS, Alex CP, Henrickson KI et al: A vancomycin-heparin lock solution for prevention of nosocomial bloodstream infection in critically ill neonates with peripherally inserted central venous catheters: A prospective, randomized trial. Pediatrics 2005;116:e198-205.

375. Dogra GK, Herson H, Hutchison B, et al: Prevention of tunneled hemodialysis catheter-related infections using catheter-restricted filling with gentamicin and citrate: A randomized controlled study. J Am Soc Nephrol 2002:13:2133-2139.

376. Raad I, Hachem R, Tcholakian RK, Sherertz R: Efficacy of minocycline and EDTA lock solution in preventing catheter-related bacteremia, septic phlebitis, and endocarditis in rabbits. Antimicrob Agents Chemother 2002;46:327-332

377. Maki DG, Crnich Cl, Safdar N Successful use of a $25 \%$ alcohol lock solution for prevention of recurrent CVC-related bloodstream infection in a patient on home TNA [abstract \#K671]. In Abstracts and Proceedings from the 42nd Interscience Conference on Antimicrobial Agents and Chemotherapy. Washington, DC American Society for Microbiology, 2002, p 320.

378. Allon M: Prophylaxis against dialysis catheter-related bacteremia with a novel antimicrobial lock solution. Clin Infect Dis 2003;36: 1539-1544.

379. Segura M, Alia C, Valverde J, et al: Assessment of a new hub design and the semiquantitative catheter culture method using an in vivo experimental model of catheter sepsis. I Clin Microbiol 1990;28:2551-2554.

380. Segura M, Alvarez-Lerma F, Tellado JM, et al: A clinical trial on the prevention of catheter-related sepsis using a new hub model. Ann Surg 1996;223: 363-369.

381. Leon C, Alvarez-Lerma F, Ruiz-Santana $S$, et al: Antiseptic chamber-containing hub reduces central venous catheterrelated infection: A prospective, randomized study. Crit Care Med 2003;31:1318-1324.

382. Luna J, Masdeu G, Perez M, et al: Clinical trial evaluating a new hub device designed to prevent catheterrelated sepsis. Eur J Clin Microbiol Infect Dis 2000;19:655-662.

383. Guidelines for the management of adults with hospital-acquired ventilator-associated, and healthcareassociated pneumonia. Am J Respir Crit Care Med 2005:171:388-416.

384. McEachern R, Campbell GD Jr: Hospital-acquired pneumonia: Epidemiology, etiology, and treatment. Infect Dis Clin North Am 1998;12. 761-779.

385. Safdar N, Dezfulian C, Collard HR, Saint S: Clinical and economic consequences of ventilator-associated pneumonia: A systematic review. Crit Care Med 2005;33:2184-2193.

386. Gross PA, Neu HC, Aswapokee P, et al: Deaths from nosocomial infections: Experience in a university hospital and a community hospital. Am I Med 1980;68:219-223.

387. Zhang P, Summer WR, Bagby G), Nelson S: Innate immunity and pulmonary host defense. Immunol Rev 2000;173:39-51.

388. Laurenzi GA, Potter RT, Kass EH: Bacteriologic flora of the lower respiratory tract. $\mathrm{N}$ Engl I Med $1961 ; 265: 1273-1278$

389. Lillehoj ER, Kim KC: Airway mucus: Its components and function. Arch Pharm Res 2002;25:770-780.

390. Salathe M, Wanner A: Nonspecific host defenses: Mucociliary clearance and cough. In Niederman M (ed): Respiratory Infections. Philadelphia, Saunders, 1994, pp 17-32.

391. Zeiher BG, Hornick DB: Pathogenesis of respiratory infections and host defenses. Curr Opin Pulm Med 1996:2:166-173.
392. Strieter RM, Belperio JA, Keane MP: Host innate defenses in the lung: The role of cytokines. Curr Opin Infect Dis 2003;16:193-198.

393. Johanson WG, Pierce AK, Sanford JP: Changing pharyngeal bacterial flora of hospitalized patients. Emergence of gram-negative bacilli. N Engl J Med 1969;281:1137-1140.

394. Sigalet DL, Mackenzie SL, Hameed SM: Enteral nutrition and mucosal immunity: implications for feeding strategies in surgery and trauma. Can J Surg 2004;47:109-116.

395. Safdar N, Crnich Cl, Maki DG: The pathogenesis of ventilator-associated pneumonia: Its relevance to developing effective strategies for prevention. Respir Care 2005;50:725739; discussion 39-41.

396. de Latorre FJ, Pont T, Ferrer A, et al: Pattern of tracheal colonization during mechanical ventilation. Am J Respir Crit Care Med 1995;152:1028-1033.

397. George DL, Falk PS, Wunderink RG, et al: Epidemiology of ventilator-acquired pneumonia based on protected bronchoscopic sampling. Am J Respir Crit Care Med 1998;158:1839-1847.

398. Ewig S, Torres A, El-Ebiary M, et al: Bacterial colonization patterns in mechanically ventilated patients with traumatic and medical head injury. Incidence, risk factors, and association with ventilator-associated pneumonia. Am J Respir Crit Care Med 1999;159: 188-198.

399. Hamill RJ, Houston ED, Georghiou PR, et al: An outbreak of Burkholderia (formerly Pseudomonas) cepacia respiratory tract colonization and infection associated with nebulized albuterol therapy. Ann Intern Med 1995; 122:762-766.

400. Estes RJ, Meduri GU: The pathogenesis of ventilator-associated pneumonia:

I. Mechanisms of bacterial transcolonization and airway inoculation. Intensive Care Med 1995;21:365-383.

401. Alcon A, Fabregas N, Torres A: Hospital-acquired pneumonia: Etiologic considerations. Infect Dis Clin North Am 2003;17:679-695.

402. Bonten MI, Gaillard CA, de Leeuw PW Stobberingh EE: Role of colonization of the upper intestinal tract in the pathogenesis of ventilator-associated pneumonia. Clin Infect Dis 1997;24: 309-319.

403. Bonten MJ, Kullberg BJ, van Dalen $\mathrm{R}$ et al: Selective digestive decontamination in patients in intensive care. The Dutch Working Group on Antibiotic Policy. I Antimicrob Chemother 2000;46: 351-362.

404. Pugin J, Auckenthaler R, Lew DP, Suter PM: Oropharyngeal decontamination decreases incidence of ventilatorassociated pneumonia. A randomized, placebo-controlled, double-blind clinical trial. JAMA 1991;265 2704-2710.

405. Bergmans DC, Bonten MJ, Gaillard CA, et al: Prevention of ventilatorassociated pneumonia by oral decontamination: A prospective, randomized, double-blind, placebocontrolled study. Am I Resp Crit Care Med 2001;164:382-388. 
406. Koeman M, van der Ven AJ, Hak E, et al: Oral decontamination with chlorhexidine reduces the incidence of ventilator-associated pneumonia. Am J Respir Crit Care Med 2006;173:1348-1355.

407. Chlebicki MP, Safdar N: Topical chlorhexidine for prevention of ventilator-associated pneumonia: A meta-analysis. Crit Care Med 2007;35:595-602.

408. Meduri GU, Estes RJ: The pathogenesis of ventilator-associated pneumonia: II. The lower respiratory tract. Intensive Care Med 1995;21:452-461.

409. Park DR: The microbiology of ventilator-associated pneumonia. Respir Care 2005;50:742-763.

410. Chastre J, Fagon JY: Ventilatorassociated pneumonia. Am J Resp Crit Care Med 2002;165:867-903.

411. Johanson WG Jr, Pierce AK, Sanford JP, Thomas GD: Nosocomial respiratory infections with gram-negative bacilli. The significance of colonization of the respiratory tract. Ann Intern Med 1972;77:701-706.

412. Fagon JY, Chastre J, Hance AJ, et al: Evaluation of clinical judgment in the identification and treatment of nosocomial pneumonia in ventilated patients. Chest 1993;103:547-553.

413. Montravers P, Fagon JY, Chastre J, et al: Follow-up protected specimen brushes to assess treatment in nosocomial pneumonia. Am Rev Respir Dis 1993; 147:38-44.

414. Cook DJ, Fitzgerald JM, Guyatt GH, Walter S: Evaluation of the protected brush catheter and bronchoalveolar lavage in the diagnosis of nosocomial pneumonia. J Intensiv Care Med 1991;6:196-205.

415. Cook DJ, Brun-Buisson C, Guyatt GH, Sibbald WJ: Evaluation of new diagnostic technologies:

Bronchoalveolar lavage and the diagnosis of ventilator-associated pneumonia. Crit Care Med 1994;22: 1314-1322.

416. Torres A, El-Ebiary M: Bronchoscopic BAL in the diagnosis of ventilatorassociated pneumonia. Chest 2000;117(4 Suppl 2):198S-202S.

417. Shorr AF, Sherner JH, Jackson WL, Kollef MH: Invasive approaches to the diagnosis of ventilator-associated pneumonia: A meta-analysis. Crit Care Med 2005;33:46-53.

418. Pugin I, Auckenthaler R, Mili N, et al: Diagnosis of ventilator-associated pneumonia by bacteriologic analysis of bronchoscopic and nonbronchoscopic "blind" bronchoalveolar lavage fluid. Am Rev Respir Dis 1991;143: 1121-1129.

419. Fabregas N, Ewig S, Torres A, et al: Clinical diagnosis of ventilator associated pneumonia revisited: Comparative validation using immediate post-mortem lung biopsies. Thorax 1999:54:867-873.

420. Fagon JY, Chastre J, Wolff M, et al: Invasive and noninvasive strategies for management of suspected ventilator-associated pneumonia. A randomized trial. Ann Intern Med 2000;132:621-630.

421. Heyland D, Dodek P, Muscedere J, Day $A$ : A randomized trial of diagnostic techniques for ventilator-associated pneumonia. N Engl J Med 2006;355: 2619-2630.

422. Kollef MH: Diagnosis of ventilatorassociated pneumonia. N Engl J Med 2006;355:2691-2693.

423. Cunnion KM, Weber DJ, Broadhead WE, et al: Risk factors for nosocomial pneumonia: Comparing adult criticalcare populations. Am J Respir Crit Care Med 1996:153:158-162.

424. Trouillet JL, Chastre J, Vuagnat A, et al: Ventilator-associated pneumonia caused by potentially drug-resistant bacteria. Am J Resp Crit Care Med 1998;157:531-539.

425. Torres A, Gatell JM, Aznar E, et al: Re-intubation increases the risk of nosocomial pneumonia in patients needing mechanical ventilation. Am J Resp Crit Care Med 1995;152: 137-141.

426. Fourrier $F$, Duvivier B, Boutigny $H$, et al: Colonization of dental plaque: A source of nosocomial infections in intensive care unit patients. Crit Care Med 1998;26:301-308.

427. du Moulin GC, Paterson DG, HedleyWhyte J, Lisbon A: Aspiration of gastric bacteria in antacid-treated patients: A frequent cause of postoperative colonisation of the airway. Lancet 1982;1:242-245.

428. Daschner F, Reuschenbach K, Pfisterer J, et al: The effect of stress ulcer prevention on the incidence of pneumonia in artificial respiration. Anaesthetist 1987;36:9-18.

429. Cook D, Guyatt G, Marshall I, et al: A comparison of sucralfate and ranitidine for the prevention of upper gastrointestinal bleeding in patients requiring mechanical ventilation. Canadian Critical Care Trials Group. N Engl I Med 1998:338:791-797.

430. Joshi N, Localio AR, Hamory BH: A predictive risk index for nosocomial pneumonia in the intensive care unit. Am J Med 1992;93:135-142.

431. Torres A, Serra-Batlles I, Ros E, et al: Pulmonary aspiration of gastric contents in patients receiving mechanical ventilation: The effect of body position. Ann Intern Med 1992;116:540-543.

432. Schweickert WD, Gehlbach BK Pohlman AS, et al: Daily interruption of sedative infusions and complications of critical illness in mechanically ventilated patients. Crit Care Med 2004;32:1272-1276.

433. Taylor RW, Manganaro L, O'Brien J, et al: Impact of allogenic packed red blood cell transfusion on nosocomial infection rates in the critically ill patient. Crit Care Med 2002;30: 2249-2254.

434. Dodek P, Keenan S, Cook D, et al: Evidence-based clinical practice guideline for the prevention of ventilator-associated pneumonia. Ann Intern Med 2004;141:305-313.

435. Kollef $\mathrm{MH}$ : The prevention of ventilator-associated pneumonia N Engl J Med 1999;340:627-634.

436. Brochard L, Mancebo J, Wysocki M, et al: Noninvasive ventilation for acute exacerbations of chronic obstructive pulmonary disease. $\mathrm{N}$ Engl J Med 1995;333:817-822.

437. Girou E, Schortgen F, Delclaux C, et al: Association of noninvasive ventilation with nosocomial infections and survival in critically ill patients. JAMA 2000;284:2361-2367.

438. Ely EW, Baker AM, Dunagan DP, et al: Effect on the duration of mechanical ventilation of identifying patients capable of breathing spontaneously. N Engl J Med 1996;335:1864-1869.

439. Kollef $M H$, Shapiro SD, Silver $P$, et al: A randomized, controlled trial of protocol-directed versus physiciandirected weaning from mechanical ventilation. Crit Care Med 1997;25: 567-574

440. Dries DJ, McGonigal MD, Malian MS, et al: Protocol-driven ventilator weaning reduces use of mechanical ventilation, rate of early reintubation, and ventilator-associated pneumonia. J Trauma 2004;56:943-951.

441. McLean SE, Jensen LA, Schroeder DG, et al: Improving adherence to a mechanical ventilation weaning protocol for critically ill adults: Outcomes after an implementation program. Am J Crit Care 2006;15: 299-309.

442. Rodriguez JL, Steinberg SM, Luchetti FA, et al: Early tracheostomy for primary airway management in the surgical critical care setting. Surgery 1990;108:655-659.

443. Drakulovic MB, Torres A, Bauer TT, et al: Supine body position as a risk factor for nosocomial pneumonia in mechanically ventilated patients: A randomised trial. Lancet 1999;354 1851-1858.

444. van Nieuwenhoven CA, Vandenbroucke-Grauls C, van Tiel FH, et al: Feasibility and effects of the semirecumbent position to prevent ventilator-associated pneumonia: $A$ randomized study. Crit Care Med 2006;34:396-402.

445. Schleder B, Stott K, Lloyd RC: The effect of a comprehensive oral care protocol on patients at risk for ventilator-associated pneumonia. I Advocate Health Care 2002;4:27-30.

446. Binkley C, Furr LA, Carrico R, McCurren C: Survey of oral care practices in US intensive care units. Am J Infect Control 2004;32:161-169.

447. Cutler Cl, Davis N: Improving oral care in patients receiving mechanical ventilation. Am I Crit Care $2005 \cdot 14 \cdot 389-394$

448. Valles J, Artigas A, Rello J, et al: Continuous aspiration of subglottic secretions in preventing ventilatorassociated pneumonia. Ann Intern Med 1995;122:179-186.

449. Kollef MH, Skubas NJ, Sundt TM: A randomized clinical trial of continuous aspiration of subglottic secretions in cardiac surgery patients. Chest 1999;116:1339-1346.

450. Cook D, Ricard JD, Reeve B, et al: Ventilator circuit and secretion management strategies: A FrancoCanadian survey. Crit Care Med 2000;28:3547-3554

451. Kirton OC, DeHaven B, Morgan I, et al: A prospective, randomized comparison of an in-line heat moisture exchange filter and heated wire humidifiers: Rates of ventilatorassociated early-onset (communityacquired) or late-onset (hospital-acquired) pneumonia and 
incidence of endotracheal tube occlusion. Chest 1997;112:1055-1059.

452. Cook D, De Jonghe B, Brochard L, Brun-Buisson C: Influence of airway management on ventilator-associated pneumonia: Evidence from randomized trials. JAMA 1998;279: 781-787.

453. Kollef $\mathrm{MH}$, Shapiro SD, Boyd V, et al: A randomized clinical trial comparing an extended-use hygroscopic condenser humidifier with heatedwater humidification in mechanically ventilated patients. Chest 1998;113: 759-767.

454. Collard HR, Saint S, Matthay MA: Prevention of ventilator-associated pneumonia: An evidence-based systematic review. Ann Intern Med 2003;138:494-501.

455. Mayhall CG: The Trach Care closed tracheal suction system: A new medical device to permit tracheal suctioning without interruption of ventilatory assistance. Infect Contro Hosp Epidemiol 1988;9:125-126.

456. Deppe SA, Kelly JW, Thoi LL, et al: Incidence of colonization, nosocomia pneumonia, and mortality in critically ill patients using a Trach Care closedsuction system versus an open-suction system: Prospective, randomized study. Crit Care Med 1990;18: 1389-1393.

457. Johnson KL, Kearney PA, Johnson SB, et al: Closed versus open endotracheal suctioning: costs and physiologic consequences. Crit Care Med 1994;22:658-666.

458. Combes P, Fauvage B, Oleyer C: Nosocomial pneumonia in mechanically ventilated patients, a prospective randomised evaluation of the Stericath closed suctioning system. Intensive Care Med 2000;26:878-882.

459. Kollef $M H$, Prentice D, Shapiro SD, et al: Mechanical ventilation with or without daily changes of in-line suction catheters. Am J Resp Crit Care Med 1997;156(2 Pt 1):466-472.

460. Bonten MJ, Gaillard CA, van der Geest $S$, et al: The role of intragastric acidity and stress ulcus prophylaxis on colonization and infection in mechanically ventilated ICU patients. A stratified, randomized, double-blind study of sucralfate versus antacids. Am J Respir Crit Care Med 1995;152: 1825-1834.

461. Thomason $\mathrm{MH}$, Payseur ES, Hakenewerth AM, et al: Nosocomial pneumonia in ventilated trauma patients during stress ulcer prophylaxis with sucralfate, antacid, and ranitidine. J Trauma 1996;41:503-508.

462. Silvestri L, Petros Al, Viviani M, et al: Selective decontamination of the digestive tract and ventilatorassociated pneumonia (part 1). Respir Care 2006;51:67-69; author reply 70-72.

463. van Saene HK, Damjanovic V, Silvestri $L$, et al: Selective decontamination of the digestive tract and ventilatorassociated pneumonia (part 2). Respir Care 2006;51:72-75.

464. Bonten MI: Selective digestive tract decontamination-will it prevent infection with multidrug-resistant gram-negative pathogens but still be applicable in institutions where methicillin-resistant Staphylococcus aureus and vancomycin-resistant enterococci are endemic? Clin Infect Dis 2006;43(Suppl 2):S70-74.

465. Bonten MJ, Krueger WA: Selective decontamination of the digestive tract: Cumulating evidence, at last? Semin Respir Crit Care Med 2006;27:18-22.

466. DeRiso AJ II, Ladowski JS, Dillon TA, et al: Chlorhexidine gluconate $0.12 \%$ oral rinse reduces the incidence of total nosocomial respiratory infection and nonprophylactic systemic antibiotic use in patients undergoing heart surgery. Chest 1996;109: 1556-1561.

467. Houston S, Hougland P, Anderson IJ, et al: Effectiveness of $0.12 \%$ chlorhexidine gluconate oral rinse in reducing prevalence of nosocomial pneumonia in patients undergoing heart surgery. Am J Crit Care 2002; 11:567-570.

468. Warren JW: The catheter and urinary tract infection. Med Clin North Am 1991;75:481-493

469. Bryan CS, Reynolds KL: Hospitalacquired bacteremic urinary tract infection: Epidemiology and outcome. J Urol 1984;132:494-498.

470. Platt R, Polk BF, Murdock B, Rosner B Reduction of mortality associated with nosocomial urinary tract infection. Lancet 1983;1:893-897.

471. Tambyah PA, Maki DG: Catheterassociated urinary tract infection is rarely symptomatic: A prospective study of 1497 catheterized patients. Arch Intern Med 2000;160:678-682.

472. Tambyah PA, Knasinski V, Maki DG: The direct costs of nosocomial catheter-associated urinary tract infection in the era of managed care. Infect Control Hosp Epidemiol 2002:23:27-31.

473. Nicolle LE: Catheter-related urinary tract infection. Drug Aging 2005;22: 627-639.

474. Wazait HD, Patel HR, Veer V, et al: Catheter-associated urinary tract infections: Prevalence of uropathogens and pattern of antimicrobial resistance in a UK hospital (1996-2001). BJU Int 2003;91:806-809.

475. Tambyah PA, Halvorson KT, Maki DG: A prospective study of pathogenesis of catheter-associated urinary tract infections. Mayo Clin Proc 1999;74: 131-136.

476. Saint S, Chenoweth CE: Biofilms and catheter-associated urinary tract infections. Infect Dis Clin North Am 2003; 17:411-432.

477. Johnson JR, Kuskowski MA, Wilt TJ: Systematic review: Antimicrobial urinary catheters to prevent catheterassociated urinary tract infection in hospitalized patients. Ann Intern Med 2006;144:116-126.

478. Maki DG, Knasinski V, Halvorson KT, et al: A prospective, randomized investigator-blinded trial of a nove nitrofurazone-impregnated urinary catheter [abstract M49]. Infect Control Hosp Epidemiol 1997;18(Suppl):50.

479. Darouiche RO, Smith JA Jr, Hanna $\mathrm{H}$, et al: Efficacy of antimicrobialimpregnated bladder catheters in reducing catheter-associated bacteriuria: A prospective, randomized, multicenter clinical trial. Urology 1999;54:976-981.

480. Saint S, Elmore JG, Sullivan SD, et al: The efficacy of silver alloy-coated urinary catheters in preventing urinary tract infection: A meta-analysis. Am J Med 1998;105:236-241.

481. Wright GD: The antibiotic resistome: The nexus of chemical and genetic diversity. Nat Rev Microbiol 2007;5: 175-186.

482. Lautenbach E, Fishman NO, Bilker WB et al: Risk factors for fluoroquinolone resistance in nosocomial Escherichia coli and Klebsiella pneumoniae infections. Arch Intern Med 2002; 162:2469-2477

483. Lautenbach E, Strom BL, Bilker WB, et al: Epidemiological investigation of fluoroquinolone resistance in infections due to extended-spectrum betalactamase-producing Escherichia coli and Klebsiella pneumoniae. Clin Infect Dis 2001;33:1288-1294.

484. Fraser GL, Stogsdill P, Dickens JD Jr, et al: Antibiotic optimization. An evaluation of patient safety and economic outcomes. Arch Intern Med 1997;157:1689-1694.

485. Solomon DH, Van Houten L, Glynn $\mathrm{RJ}$, et al: Academic detailing to improve use of broad-spectrum antibiotics at an academic medical center. Arch Intern Med 2001;161: 1897-1902.

486. Pear SM, Williamson TH, Bettin KM, et al: Decrease in nosocomial Clostridium difficile-associated diarrhea by restricting clindamycin use. Ann Intern Med 1994;120:272-277.

487. Quale I, Landman D, Saurina G, et al: Manipulation of a hospital antimicrobial formulary to control an outbreak of vancomycin-resistant enterococci. Clin Infect Dis 1996;23: 1020-1025.

488. Lautenbach E, LaRosa LA, Marr AM, et al: Changes in the prevalence of vancomycin-resistant enterococci in response to antimicrobial formulary interventions: Impact of progressive restrictions on use of vancomycin and third-generation cephalosporins. Clin Infect Dis 2003;36:440-446.

489. Bantar C, Sartori B, Vesco E, et al: A hospitalwide intervention program to optimize the quality of antibiotic use: Impact on prescribing practice, antibiotic consumption, cost savings, and bacterial resistance. Clin Infect Dis 2003;37:180-186.

490. Warren DK, Hill HA, Merz LR, et al: Cycling empirical antimicrobial agents to prevent emergence of antimicrobial-resistant gram-negative bacteria among intensive care unit patients. Crit Care Med 2004;32 2450-2456.

491. Merz LR, Warren DK, Kollef MH, et al: The impact of an antibiotic cycling program on empirical therapy for gram-negative infections. Chest 2006 130:1672-1678

492. McGowan JE Jr: Minimizing antimicrobial resistance in hospital bacteria: Can switching or cycling drugs help? Infect Control 1986;7:573-576.

493. Gerding DN: Antimicrobial cycling: lessons learned from the aminoglycoside experience. Infect 
Control Hosp Epidemiol 2000;21(1 Suppl):S12-17.

494. Bonhoeffer S, Lipsitch M, Levin BR: Evaluating treatment protocols to prevent antibiotic resistance. Proc Natl Acad Sci U S A 1997;94:12106-12111.

495. Durbin WA Jr, Lapidas B, Goldmann DA: Improved antibiotic usage following introduction of a novel prescription system. JAMA 1981;246:1796-1800.

496. Kollef $\mathrm{MH}$ : Hospital-acquired pneumonia and de-escalation of antimicrobial treatment. Crit Care Med 2001;29:1473-1475.

497. Kollef $\mathrm{MH}$ : Providing appropriate antimicrobial therapy in the intensive care unit: Surveillance vs. deescalation. Crit Care Med 2006;34:903-905.

498. Burke JP, Pestotnik SL: Antibiotic use and microbial resistance in intensive care units: Impact of computerassisted decision support. J Chemother 1999;11:530-535

499. Evans RS, Classen DC, Pestotnik SL, et al: A decision support tool for antibiotic therapy. Proc Annu Symp Comput Appl Med Care 1995:651-655.

500. Stoutenbeek CP, van Saene HK: Prevention of pneumonia by selective decontamination of the digestive tract (SDD). Intensive Care Med 1992:18(Suppl 1):S18-23.

501. van Saene HK, Stoutenbeek CC, Stoller JK: Selective decontamination of the digestive tract in the intensive care unit: Current status and future prospects. Crit Care Med 1992:20:691-703.

502. Vandenbroucke-Grauls CM, Vandenbroucke JP: Effect of selective decontamination of the digestive tract on respiratory tract infections and mortality in the intensive care unit. Lancet 1991;338:859-862.

503. Meta-analysis of randomised controlled trials of selective decontamination of the digestive tract. Selective Decontamination of the Digestive Tract Trialists' Collaborative Group. BMJ 1993;307:525-532.

504. Kollef $\mathrm{MH}$ : The role of selective digestive tract decontamination on mortality and respiratory tract infections. A meta-analysis. Chest 1994:105:1101-1108.

505. Heyland DK, Cook DJ, Jaeschke R, et al: Selective decontamination of the digestive tract. An overview. Chest 1994;105:1221-1229.

506. Hurley JC: Prophylaxis with enteral antibiotics in ventilated patients: Selective decontamination or selective cross-infection? Antimicrob Agents Chemother 1995;39:941-947.

507. D'Amico R, Pifferi S, Leonetti C, et al: Effectiveness of antibiotic prophylaxis in critically ill adult patients: Systematic review of randomised controlled trials. BMI 1998;316: 1275-1285.

508. Nathens AB, Marshall IC: Selective decontamination of the digestive tract in surgical patients: A systematic review of the evidence. Arch Surg 1999; 134:170-176.

509. Safdar N, Said A, Lucey MR: The role of selective digestive decontamination for reducing infection in patients undergoing liver transplantation: A systematic review and meta-analysis Liver Transpl 2004;10:817-827.

510. Liberati A, D'Amico R, Pifferi, et al: Antibiotic prophylaxis to reduce respiratory tract infections and mortality in adults receiving intensive care. Cochrane Database Syst Rev 2004(1):CD000022.

511. Silvestri $L$, van Saene $H K$, Milanese $M$, Gregori D: Impact of selective decontamination of the digestive tract on fungal carriage and infection: Systematic review of randomized controlled trials. Intensive Care Med 2005;31:898-910.

512. Silvestri L, van Saene HK, Milanese M, et al: Selective decontamination of the digestive tract reduces bacterial bloodstream infection and mortality in critically ill patients. Systematic review of randomized, controlled trials. J Hosp Infect 2007;65:187-203.

513. van Nieuwenhoven CA, Buskens $E$, van Tiel FH, Bonten MJ: Relationship between methodological trial quality and the effects of selective digestive decontamination on pneumonia and mortality in critically ill patients. JAMA 2001;286:335-340.

514. Lingnau W, Berger J, Javorsky $F$, et al: Changing bacterial ecology during a five-year period of selective intestinal decontamination. J Hosp Infect 1998:39:195-206.

515. Leone M, Albanese J, Antonini F, et al: Long-term (6-year) effect of selective digestive decontamination on antimicrobial resistance in intensive care, multiple-trauma patients. Crit Care Med 2003:31:2090-2095.

516. Saunders GL, Hammond JM, Potgieter PD, et al: Microbiological surveillance during selective decontamination of the digestive tract (SDD). I Antimicrob Chemother 1994;34:529-544.

517. Paterson DL, Singh N, Rihs JD, et al: Control of an outbreak of infection due to extended-spectrum betalactamase-producing Escherichia coli in a liver transplantation unit. Clin Infect Dis 2001:33:126-128.

518. Dezfulian C, Shojania K, Collard HR, et al: Subglottic secretion drainage for preventing ventilator-associated pneumonia: A meta-analysis. Am J Med 2005;118:11-18.

519. Haley RW, Bregman DA: The role of understaffing and overcrowding in recurrent outbreaks of staphylococcal infection in a neonatal special-care unit. J Infect Dis 1982;145:875-885.

520. Levine AS, Siegel SE, Schreiber AD et al: Protected environments and prophylactic antibiotics. A prospective controlled study of their utility in the therapy of acute leukemia. $\mathrm{N}$ Engl J Med 1973;288:477-483.

521. Evans HE, Akpata SO, Baki A, Behrman RE: Bacteriologic and clinical evaluation of gowning in a premature nursery. J Pediatr 1971;78:883-886.

522. Agbayani M, Rosenfeld W, Evans $H$, et al: Evaluation of modified gowning procedures in a neonatal intensive care unit. Am J Dis Child 1981;135: 650-652.

523. Donowitz LG: Failure of the overgown to prevent nosocomial infection in a pediatric intensive care unit. Pediatrics 1986;77:35-38
524. Artru F, Brun Y, Firholz P, Deleuze R: Prevention of hospital-acquired infection. Efficacy of isolation procedures in a neurological intensive care unit (ICU). Nouv Presse Med 1979;8:1065-1069.

525. Maki DG, Zilz MA, McComick R: The effectiveness of using preemptive barrier precautions routinely (protective isolation) in all high-risk patients to prevent nosocomial infection with resistant organisms, especially MRSA, VRE and C. difficile. In Abstracts and Proceedings of the 34th Annual Meeting of the Infectious Disease Society of North America; 1996. New Orleans, Infectious Disease Society of America, 1996.

526. van Voorhis J, Destefano L, Sobek S, et al: Impact of barrier precautions and cohorting on a monoclonal outbreak of vancomycin-resistant enterococcus faecium (VRE). In Abstracts and Proceedings of the 7th Annual Meeting of the Society for Healthcare Epidemiology of America; 1997. St. Louis, Society for Healthcare Epidemiology of America, 1997.

527. Klein BS, Perloff WH, Maki DG: Reduction of nosocomial infection during pediatric intensive care by protective isolation. $\mathrm{N}$ Engl J Med 1989;320:1714-1721.

528. Slota M, Green M, Farley A, et al: The role of gown and glove isolation and strict handwashing in the reduction of nosocomial infection in children with solid organ transplantation. Crit Care Med 2001;29:405-412.

529. McManus AT, Goodwin CW, Pruitt BA Ir: Observations on the risk of resistance with the extended use of vancomycin. Arch Surg 1998;133:1207-1211.

530. Koss WG, Khalili TM, Lemus JF, et al: Nosocomial pneumonia is not prevented by protective contact isolation in the surgical intensive care unit. Am Surg 2001;67:1140-1144.

531. Slaughter S, Hayden MK, Nathan C, et al: A comparison of the effect of universal use of gloves and gowns with that of glove use alone on acquisition of vancomycin-resistant enterococci in a medical intensive care unit. Ann Intern Med 1996; 125:448-456.

532. Safdar N, Marx I, Meyer N, Maki DG: The effectiveness of preemptive enhanced barrier precautions for controlling MRSA in a burn unit. In Abstracts and Proceedings of the 43rd InterScience Conference on Antimicrobial Agents and Chemotherapy; 2003. Chicago, American Society of Microbiology, 2003

533. Srinivasan A, Song $X$, Ross $T$, et al: A prospective study to determine whether cover gowns in addition to gloves decrease nosocomial transmission of vancomycin-resistant enterococci in an intensive care unit. Infect Control Hosp Epidemiol 2002;23:424-428

534. Montecalvo MA, Jarvis WR, Uman J, et al: Infection-control measures reduce transmission of vancomycinresistant enterococci in an endemic setting. Ann Intern Med 1999;131:269-272. 
535. Morris JG Jr, Shay DK, Hebden JN et al: Enterococci resistant to multiple antimicrobial agents, including vancomycin. Establishment of endemicity in a university medical center. Ann Intern Med 1995;123: 250-259.

536. Castaldo P, Stratta RJ, Wood RP, et al: Clinical spectrum of fungal infections after orthotopic liver transplantation. Arch Surg 1991;126:149-156.

537. Maki DG, Ringer M: Evaluation of dressing regimens for prevention of infection with peripheral intravenous catheters. Gauze, a transparent polyurethane dressing, and an iodophor-transparent dressing. JAMA 1987:258:2396-2403.

538. Patterson WB, Craven DE, Schwartz DA, et al: Occupational hazards to hospital personnel. Ann Intern Med 1985; 102:658-680.

539. Gestal J]: Occupational hazards in hospitals: Risk of infection. $\mathrm{Br}$ J Ind Med 1987;44:435-442.

540. Rogers B: Health hazards in nursing and health care: An overview. Am J Infect Control 1997;25:248-261.

541. Nora Ir II, Doebbeling BN: New vaccines and vaccination programs for hospital staff members. In Wenzel RP (ed): Prevention and Control of Nosocomial Infections, 4th ed. Philadelphia, Lippincott Williams \& Wilkins, 2003, pp 413-429.

542. Immunization of health-care workers: Recommendations of the Advisory Committee on Immunization Practices (ACIP) and the Hospital Infection Control Practices Advisory Committee (HICPAC). MMWR Recomm Rep 1997;46(RR-18): 1-42.

543. McCormick RD, Meisch MG, Ircink FG, Maki DG: Epidemiology of hospital sharps injuries: A 14-year prospective study in the pre-AIDS and AIDS eras. Am J Med 1991;91:301S-307S.

544. Lee JM, Botteman MF, Nicklasson L, et al: Needlestick injury in acute care nurses caring for patients with diabetes mellitus: A retrospective study. Curr Med Res Opin 2005;21: 741-747.

545. Haiduven DI, DeMaio TM, Stevens DA: A five-year study of needlestick injuries: Significant reduction associated with communication, education, and convenient placement of sharps containers. Infect Control Hosp Epidemiol 1992;13:265-271.

546. Tuma S, Sepkowitz KA: Efficacy of safety-engineered device implementation in the prevention of percutaneous injuries: A review of published studies. Clin Infect Dis 2006:42:1159-1170.

547. Goldwater PN, Law R, Nixon AD, et al: Impact of a recapping device on venepuncture-related needlestick injury. Infect Control Hosp Epidemiol 1989:10:21-25

548. McCormick RD, Maki DG: Epidemiology of needle-stick injuries in hospital personnel. Am J Med 1981;70:928-932.

549. van Loon FP, Holmes SI, Sirotkin BI, et al: Mumps surveillance-United States, 1988-1993. MMWR CDC Surveill Summ 1995;44:1-14.

550. Centers for Disease Control and Prevention: Epidemiology of measles-
United States, 2001-2003. MMWR Morb Mortal Wkly Rep 2004;53: 713-716.

551. Centers for Disease Control and Prevention: Elimination of rubella and congenital rubella syndrome-United States, 1969-2004. MMWR Morb Mortal Wkly Rep 2005;54:279-282.

552. Centers for Disease Control and Prevention: Measles_-United States, 2005. MMWR Morb Mortal Wkly Rep 2006;55:1348-1351.

553. Centers for Disease Control and Prevention: Update: Multistate outbreak of mumps-United States, lanuary 1-May 2, 2006. MMWR Morb Mortal Wkly Rep 2006;55:559-563.

554. Davis RM, Orenstein WA, Frank JA Jr, et al: Transmission of measles in medical settings. 1980 through 1984 . JAMA 1986:255:1295-1298.

555. Watson JC, Hadler SC, Dykewicz CA, et al: Measles, mumps, and rubellavaccine use and strategies for elimination of measles, rubella, and congenital rubella syndrome and control of mumps: Recommendations of the Advisory Committee on Immunization Practices (ACIP). MMWR Recomm Rep 1998;47:1-57.

556. Weber DJ, Rutala WA, Hamilton $\mathrm{H}$ : Prevention and control of varicellazoster infections in healthcare facilities. Infect Control Hosp Epidemiol 1996:17:694-705

557. Choo PW, Donahue JG, Manson JE, Platt R: The epidemiology of varicella and its complications. I Infect Dis 1995; 172:706-712.

558. Enders G, Miller E, Cradock-Watson J, et al: Consequences of varicella and herpes zoster in pregnancy: Prospective study of 1739 cases. Lancet 1994:343:1548-1551.

559. Prevention of varicella: Recommendations of the Advisory Committee on Immunization Practices (ACIP). Centers for Disease Control and Prevention. MMWR Recomm Rep 1996:45:1-36.

560. Prevention of varicella. Update recommendations of the Advisory Committee on Immunization Practices (ACIP). MMWR Recomm Rep 1999:48:1-5.

561. Bridges CB, Harper SA, Fukuda K, et al: Prevention and control of influenza. Recommendations of the Advisory Committee on Immunization Practices (ACIP). MMWR Recomm Rep 2003;52:1-34; quiz CE1-4

562. Salgado CD, Farr BM, Hall KK, Hayden FG: Influenza in the acute hospital setting. Lancet Infect Dis 2002;2: 145-155.

563. Stott Dl, Kerr G, Carman WF: Nosocomial transmission of influenza. Occup Med 2002;52:249-253.

564. Dash GP, Fauerbach L, Pfeiffer I, et al: APIC position paper: Improving health care worker influenza immunization rates. Am J Infect Control 2004;32: 123-125.

565. Nichol KL, Lind A, Margolis KL, et al: The effectiveness of vaccination against influenza in healthy, working adults. N Engl! Med 1995:333:889-893.

566. Wilde JA, McMillan JA, Serwint J, et al: Effectiveness of influenza vaccine in health care professionals: A randomized trial. JAMA 1999;281: 908-913.

567. Nichol KL, Hauge M: Influenza vaccination of healthcare workers. Infect Control Hosp Epidemiol 1997; 18:189-194.

568. Canning HS, Phillips I, Allsup S: Health care worker beliefs about influenza vaccine and reasons for nonvaccination-a cross-sectional survey. J Clin Nurs 2005; 14:922-925.

569. Carman WF, Elder AG, Wallace LA, et al: Effects of influenza vaccination of health-care workers on mortality of elderly people in long-term care: A randomised controlled trial. Lancet 2000;355:93-97.

570. Salgado CD, Giannetta ET, Hayden FG, Farr BM: Preventing nosocomial influenza by improving the vaccine acceptance rate of clinicians. Infect Control Hosp Epidemiol 2004;25: 923-928.

571. Hayward AC, Harling R, Wetten S, et al: Effectiveness of an influenza vaccine programme for care home staff to prevent death, morbidity, and health service use among residents: Cluster randomised controlled trial. BMJ 2006;333:1241.

572. Burls $A$, Jordan $R$, Barton $P$, et al: Vaccinating healthcare workers against influenza to protect the vulnerable-is it a good use of healthcare resources? A systematic review of the evidence and an economic evaluation. Vaccine 2006;24:4212-4221.

573. Walker Fl, Singleton IA, Lu P, et al: Influenza vaccination of healthcare workers in the United States, 19892002. Infect Control Hosp Epidemiol 2006;27:257-265

574. Hoffmann Cl, Perl TM: The next battleground for patient safety: Influenza immunization of healthcare workers. Infect Control Hosp Epidemiol 2005;26:850-851.

575. Wendelboe AM, Van Rie A, Salmaso S, Englund JA: Duration of immunity against pertussis after natural infection or vaccination. Pediatr Infect Dis ! 2005;24(5 Suppl):S58-61.

576. Centers for Disease Control and Prevention: Pertussis outbreak among adults at an oil refinery-lllinois, August-October 2002. MMWR Morb Mortal Wkly Rep 2003;52:1-4.

577. Calugar A, Ortega-Sanchez IR, Tiwari $T$, et al: Nosocomial pertussis: Costs of an outbreak and benefits of vaccinating health care workers. Clin Infect Dis 2006;42:981-988.

578. Ward A, Caro I, Bassinet $L$, et al: Health and economic consequences of an outbreak of pertussis among healthcare workers in a hospital in France. Infect Control Hosp Epidemiol 2005; $26: 288-292$

579. Rusk I: ACIP recommends Tdap vaccine for health care workers. Infectious Disease News 2006. Available at http:// www.infectiousdiseasenews.com/ 200603/frameset.asp?article-tdap.asp (Accessed July 5, 2007)

580. Adams G, Stover BH, Keenlyside RA, et al: Nosocomial herpetic infections in a pediatric intensive care unit. Am Epidemiol 1981;113:126-132.

581. Klotz RW: Herpetic whitlow: An occupational hazard. AANA ! 1990;58:8-13. 
582. Glassroth J, Crnich Cl: Pulmonary infections caused by mycobacterial species. In Crapo JD, Glassroth J, Karlinsky JB, King TE (eds): Baum's Textbook of Pulmonary Diseases, 7th ed. Philadelphia, Lippincott Williams \& Wilkins, 2003.

583. Butt AA, Singh N: Hepatitis C: Prevention, therapy, and role of transplantation. In Wenzel RP (ed): Prevention and Control of Nosocomial Infections, 4th ed. Philadelphia, Lippincott Williams \& Wilkins, 2003 pp 215-228

584. Jagger I, De Carli G, Perry IL, et al: Occupational exposure to blood-borne pathogens: Epidemiology and prevention. In Wenzel RP (ed): Prevention and Control of Nosocomial Infections, 4th ed. Philadelphia, Lippincott Williams \& Wilkins, 2003 pp 430-466.

585. Polish LB, Tong MJ, Co RL, et al: Risk factors for hepatitis $C$ virus infection among health care personnel in a community hospital. Am J Infect Control 1993:21:196-200.

586. Wiegand J, Buggisch P, Boecher W, et al: Early monotherapy with pegylated interferon alpha- $2 b$ for acute hepatitis $C$ infection: The HEP NET acute-HCV-II study. Hepatology 2006;43:250-256

587. Jaeckel E, Cornberg M, Wedemeyer $\mathrm{H}$, et al: Treatment of acute hepatitis $C$ with interferon alfa-2b. N Engl J Med 2001;345:1452-1457.

588. Centers for Disease Control and Prevention: Surveillance of healthcare personnel with HIV/AIDS, as of December 2002. Available at http:// www.cdc.gov/ncidod/dhqp/bp_hiv_ hp_with.html Accessed January 15, 2007.

589. Oksenhendler E, Harzic M, Le Roux IM, et al: HIV infection with seroconversion after a superficial needlestick injury to the finger. $\mathrm{N}$ Engl Med 1986;315:582.

590. Black RJ: Animal studies of prophylaxis. Am J Med 1997;102:39-44.

591. Cardo DM, Culver DH, Ciesielski CA, et al: A case-control study of HIV seroconversion in health care workers after percutaneous exposure. Centers for Disease Control and Prevention Needlestick Surveillance Group. N Engl Med 1997;337:1485-1490.

592. Panlilio AL, Cardo DM, Grohskopf LA, et al: Updated U.S. Public Health Service guidelines for the management of occupational exposures to HIV and recommendations for postexposure prophylaxis. MMWR Recomm Rep 2005; $54: 1-17$

593. Maki DG: Risk factors for nosocomial infection in intensive care. "Devices vs. nature" and goals for the next decade. Arch Intern Med 1989;149:30-35

594. Decker LA, Gross A, Miller FC, et al: $A$ rapid method for the presurgical cleansing of hands. Obstet Gynecol 1978;51:115-117.

595. Vesley D, Langholz AC, Timmermann TA: Evaluation of a mechanical handwashing device with germicidal and non-germicidal products. In Program and Abstracts of the 15th Annual Meeting of the Association for the Professionals in Infection Control; 1988. Dallas, Association for the Professionals of Infection Control, 1988.

596. Maki DG, McCormick R, Alvarado CJ, et al: Clinical evaluation of the degerming efficacy of seven agents for handwashing in hospitals. In Program and Abstracts of the 24th Interscience Conference on Antimicrobial Agents and Chemotherapy; 1984.

Washington, DC, American Society for Microbiology, 1984

597. Morrison AJ Jr, Gratz J, Cabezudo I, Wenzel RP: The efficacy of several new handwashing agents for removing non-transient bacterial flora from

hands. Infect Control 1986;7:268-272

598. Soifer N, Edlin B, Weinstein R, Group MIS: A randomized IV team trial [abstract]. In Abstracts and Proceedings from the 29th Interscience Conference on Antimicrobial Agents and Chemotherapy. Washington, DC American Society of Medicine, 1989, p 1076.

599. Hemming VG, Overall JC Jr, Britt MR: Nosocomial infections in a newborn intensive-care unit. Results of forty-one months of surveillance. $\mathrm{N}$ Engl J Med 1976:294:1310-1316.

600. Kirkland KB, Briggs JP, Trivette SL, et al: The impact of surgical-site infections in the 1990s: Attributable mortality, excess length of hospitalization, and extra costs. Infect Control Hosp Epidemiol 1999;20:725-730

601. Whitehouse JD, Friedman ND, Kirkland $K B$, et al: The impact of surgical-site infections following orthopedic surgery at a community hospital and a university hospital: Adverse quality of life, excess length of stay, and extra cost. Infect Control Hosp Epidemiol 2002;23:183-189.

602. Hollenbeak CS, Murphy DM, Koenig $S$, et al: The clinical and economic impact of deep chest surgical site infections following coronary artery bypass graft surgery. Chest 2000;118:397-402.

603. McGarry SA, Engemann II, Schmader $K$, et al: Surgical-site infection due to
Staphylococcus aureus among elderly patients: Mortality, duration of hospitalization, and cost. Infect Control Hosp Epidemio

2004;25:461-467

604. Herwaldt LA, Cullen JJ, Scholz D, et al: A prospective study of outcomes, healthcare resource utilization, and costs associated with postoperative nosocomial infections. Infect Contro Hosp Epidemiol 2006;27:1291-1298

605. Fagon JY, Chastre J, Hance AJ, et al: Nosocomial pneumonia in ventilated patients: A cohort study evaluating attributable mortality and hospital stay. Am J Med 1993;94:281-288.

606. Heyland DK, Cook DJ, Griffith L, et al: The attributable morbidity and mortality of ventilator-associated pneumonia in the critically ill patient. The Canadian Critical Trials Group. Am J Respir Crit Care Med 1999;159:1249-1256.

607. Bercault N, Boulain T: Mortality rate attributable to ventilator-associated nosocomial pneumonia in an adult intensive care unit: A prospective casecontrol study. Crit Care Med 2001;29:2303-2309.

608. Rello J, Ollendorf DA, Oster G, et al: Epidemiology and outcomes of ventilator-associated pneumonia in a large US database. Chest 2002;122:2115-2121

609. Warren DK, Shukla SJ, Olsen MA et al: Outcome and attributable cost of ventilator-associated pneumonia among intensive care unit patients in a suburban medical center. Crit Care Med 2003;31:1312-1317.

610. Cocanour CS, Ostrosky-Zeichner L Peninger $\mathrm{M}$, et al: Cost of a ventilatorassociated pneumonia in a shock trauma intensive care unit. Surg Infect 2005;6:65-72

611. Slonim AD, Kurtines HC, Sprague BM, Singh N: The costs associated with nosocomial bloodstream infections in the pediatric intensive care unit. Pediatr Crit Care Med 2001;2: 170-174.

612. Warren DK, Quadir WW, Hollenbeak CS, et al: Attributable cost of catheterassociated bloodstream infections among intensive care patients in a nonteaching hospital. Crit Care Med 2006; 34:2084-2089.

613. Mullett MD, Cook EF, Gallagher R: Nosocomial sepsis in the neonatal intensive care unit. I Perinatol 1998;18:112-115.

614. McManus AT, Mason AD Jr, McManus WF, Pruitt BA Jr: A decade of reduced gram-negative infections and mortality associated with improved isolation of burned patients. Arch Surg 1994;129:1306-1309. 